\title{
THE ESTIMATION OF STATURE FROM THE TARSALS: ENHANCING THE DISASTER VICTIM IDENTIFICATION PROCESS IN THAILAND USING THE CALCANEUS AND THE TALUS
}

\author{
By \\ Shelby Scott \\ A Thesis Submitted to \\ Saint Mary's University, Halifax, Nova Scotia \\ in Partial Fulfillment of the Requirements for \\ the Degree of Master of Science in Applied Science
}

March 2018 Halifax, Nova Scotia

(C) Shelby Scott, 2018

Approved: Dr. Tanya Peckmann

Supervisor

Department of Anthropology

Saint Mary's University

Approved: Dr. Bradley Adams

External Examiner

Director of Forensic Anthropology

Office of Chief Medical Examiner, New York

Approved: Dr. Michelle Patriquin

Supervisory Committee Member

Department of Biology

Saint Mary’s University

Approved: Dr. Claudia Garrido Varas

Supervisory Committee Member

Forensic Advisor

International Committee of the Red Cross

Date: March 9, 2018 


\section{ACKNOWLEGMENTS}

This thesis could not have been completed without the support and copious contributions of numerous people and institutions. The quality of this research was significantly enhanced by the kindly contributions of my thesis committee. First and foremost, I express gratitude to my thesis committee supervisor, Dr. Tanya Peckmann for her direction, supervision, devotion, and patience (during the countless hours spent reading and revising my work). As a teacher and an advisor, you have provided invaluable experiences and opportunities that have helped to shape my career, and I have grown so much as a scholar, teacher, and a professional under your tutelage. I also wish to thank the supporting members of my thesis committee, Dr. Michelle Patriquin and Dr. Claudia Garrido-Varas for their expertise and feedback. During my time at Saint Mary's University, Michelle has become both a mentor and a friend. So often I pushed forward with revived motivation after our impromptu discussions; you have been a valuable and positive resource throughout my degree. Thank you is also extended to Dr. Claudia Garrido-Varas, who was able to provide unique insight into this research based on her practical experience as a forensic advisor in Thailand during the 2004 'Boxing Day' tsunami. Thank you, Claudia, for agreeing to serve on my committee, and for taking the time to review my work while abroad.

My work is also indebted to a number of outside institutions and individuals who have made this research possible. Foremost, I would like to thank the individuals who have donated their bodies to medicine, without whom my research would not be possible. I would like to thank the Excellence in Osteology Research and Training Center (ORTC) and Chiang Mai University in Chiang Mai, Thailand, for allowing access to their skeletal 
collection for this research. Specifically, I would like to thank Dr. Pasuk

Mahakkanukrauh and Sittiporn Ruengdit for their assistance during data collection, and for their hospitality. Thank you also to Alice Brown (MSc), who volunteered her time as a research assistant. A sincere thank you is also extended to Dr. Susan Meek, Dr. Colleen Barber, and Dr. Jeremy Lundholm, who provided statistical advice throughout this research. Thank you, Colleen, for allowing me the opportunity to sit in on your lectures and learn biostatistics. Special thanks to Dr. Susan Meek, who has been more then generous with her time over the years, guiding me through statistical analyses and interpretation on more than one occasion, and patiently answering my constant barrage of questions. Susan, your assistance has immeasurably enriched my research. Finally, thank you to my family and friends for their unwavering support, encouragement, and willingness to lend an ear or share a drink.

Lastly, I would like to acknowledge those who have provided financial support for this research. I would like to thank the selection committees of the Canadian Society of Forensic Science (CSFS) Education Award, the Vice-President Academic \& Research International Mobility Award, and the International Development Initiative Fund through Saint Mary's University Students' Association, whose awards assisted in funding this research. Also, gratitude is extended to the Saint Mary's University Faculty of Graduate Studies and Research for providing grants to support this research, and the Saint Mary's University SMUWorks Program for their financial assistance. 


\title{
ABSTRACT \\ The Estimation of Stature from the Tarsals: Enhancing the Victim Identification Process in Thailand using the Calcaneus and the Talus
}

\author{
by Shelby Scott
}

The impact of climate change is estimated to be particularly severe in Thailand. Therefore, there is an urgent need for population-specific methodologies for the identification of unknown human remains in Thailand. The current research study focuses on the calcaneus and the talus to establish an accurate method of living stature estimation for a contemporary Thai population.

Paired calcanei and tali of 232 adult skeletonized individuals (115 males, 117 females), ranging from 19 to 96 years of age were studied from the contemporary Chiang Mai skeletal collection. Nine measurements were collected from each calcaneus: MAXL, MAXH, CFH, BH, MINB, LAL, MIDB, DAFB, DAFL. One measurement, MTAL, was collected from each talus.

All measurement variables of the calcaneus and the talus exhibit sexual dimorphism. Regression formulae, generated from other populations, were found not to be accurate predictors of stature when applied to the contemporary Thai population. Comparisons of calcaneal and talar size to other populations were made. There were significant differences for the calcaneus and the talus between the contemporary Thai and comparative populations. Therefore, population-specific regression formulae are necessary to accurately estimate stature in a contemporary Thai population. The results of the current research study will assist in estimating living stature of unknown contemporary Thai individuals, and will be useful in cases such as natural disasters where only fragmented remains are available for examination.

Date: March 9, 2018 
ACKNOWLEGMENTS ........................................................................................ ii

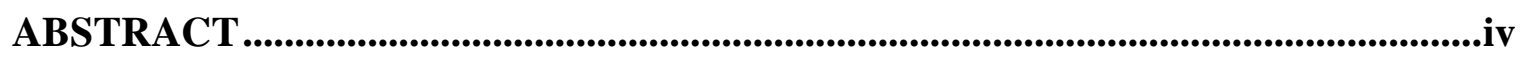

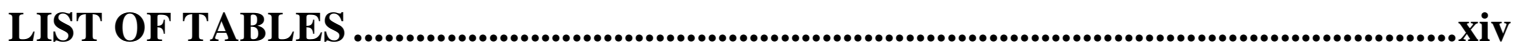

LIST OF FIGURES

CHAPTER 1: INTRODUCTION................................................................................................1

$1.1 \quad$ Research Objectives ............................................................................

1.2 Potential for Stature Estimation from the Calcaneus and the Talus ..................2

1.3 Concepts of Identity and Terminology in Forensic Anthropology .....................4

1.3.1 Differences between Sex and Gender ...........................................................

1.3.2 The Origin and Construction of 'Race' in Physical Anthropology ...............6

1.4 Forensic Anthropology and Disaster Victim Identification in Thailand...........10

1.5 Skeletal Collection: Forensic Osteology Research Center, Chiang Mai

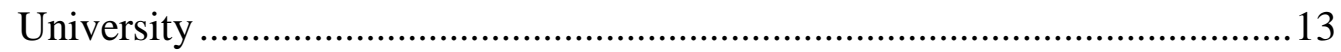

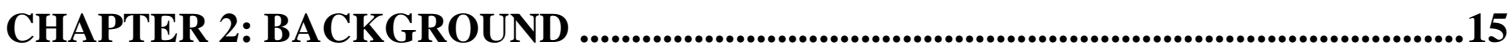

2.1 The Skeletal System and Anatomy of the Human Foot ...................................15

2.1.1 Calcaneus and Talus: Growth and Development .........................................18

2.1.2 Calcaneus: Anatomy and Function ...........................................................19

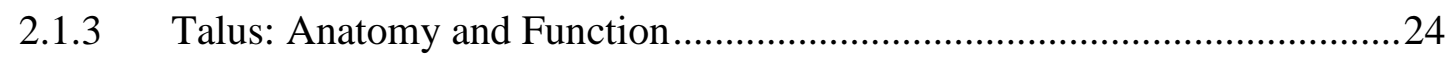

2.2 Methods of Stature Estimation in Forensic Anthropology ............................27

2.2.1 Stature Estimation: Regression Formulae from the Long Bones ........................32

2.2.2 Stature Estimation: Regression Formulae from the Skull ...................................33

2.2.3 Stature Estimation: Regression Formulae from the Vertebrae ...........................35

2.2.4 The Potential for Stature Estimation from the Calcaneus and the Talus

2.3 Climate Change in Thailand: Implications for Forensic Anthropology ...........48

2.4 The Admissibility of Forensic Anthropology Methods in Court......................50 
CHAPTER 3: MATERIALS AND METHODS .............................................................56

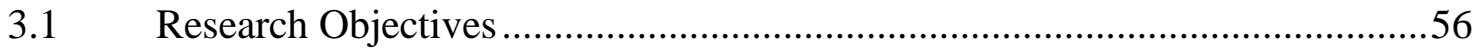

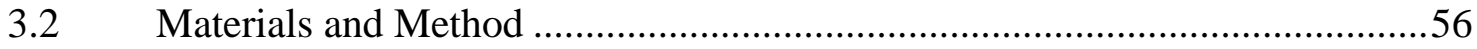

3.2.1 Demographics of Skeletal Materials Utilized for this Research.........................56

3.2.2 Skeletal Materials Excluded from this Research..............................................59

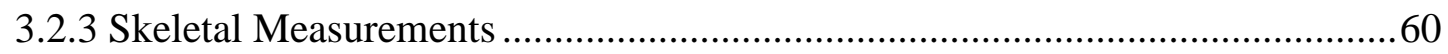

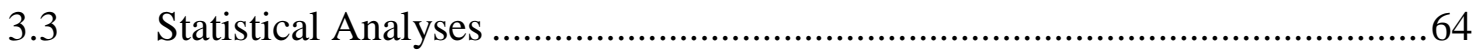

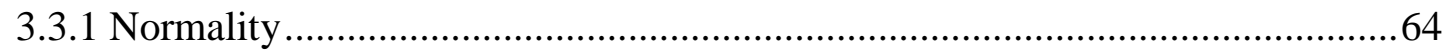

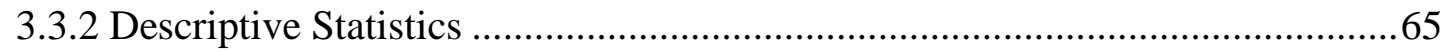

3.3.3 Analysis of Side Differences in the Thai Population ........................................66

3.3.4 Analysis of Sexual Dimorphism in the Thai Population ....................................66

3.3.5 Analysis of Observer Error and Reproducibility ................................................67

3.3.6 Simple and Multiple Regression Analyses ........................................................70

3.3.7 Cross-Validation of Thai Regression Equations ................................................77

3.3.8 Population Comparison ………………………………................................78

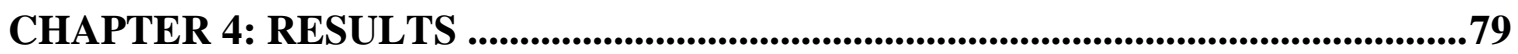

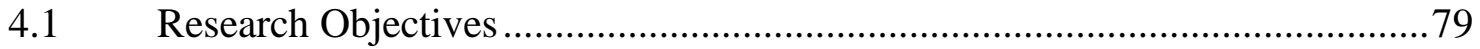

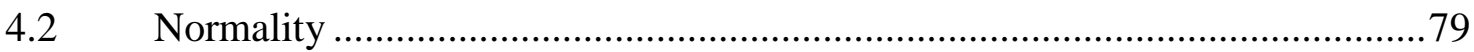

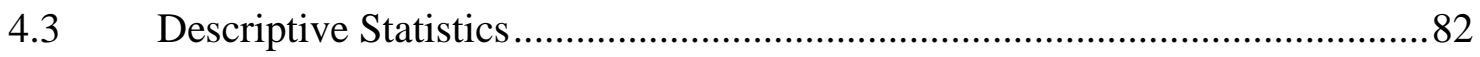

4.4 Analysis of Sexual Dimorphism in the Thai Population..................................85

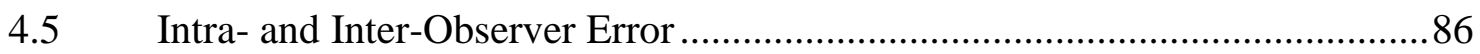

4.6 Simple and Multiple Regression Analyses........................................................92

4.6.1 Accuracy of the Bidmos and Asala (2005) Calcaneal Regression

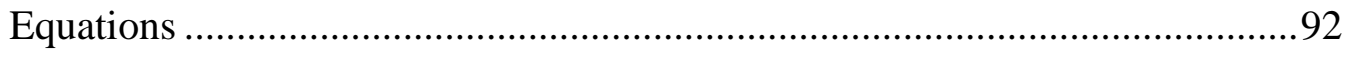

4.6.2 Accuracy of the Holland (1995) Talar Regression Equations ...........................98

4.6.3 Accuracy of Simple and Multiple Regression Equations for the Thai

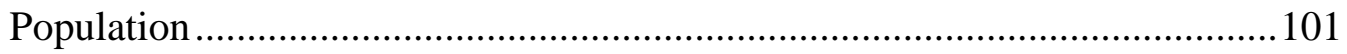

4.7 Cross-Validation of the Thai Regression Equations ……….................................112

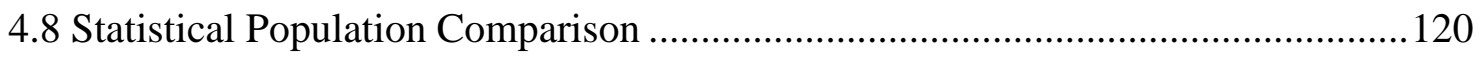

CHAPTER 5: DISCUSSION .............................................................................131

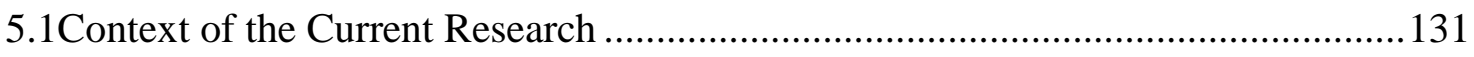


5.2 Analysis of Sexual Dimorphism in the Thai Population

5.3 Analysis of Observer Error and Reproducibility

5.4 Simple and Multiple Regression Equations

5.4.1 Accuracy of the Bidmos and Asala (2005) calcaneal regression equations

5.4.2 Accuracy of the Holland (1995) talar regression equations

5.4.3 Accuracy of simple and multiple regression equations for the contemporary Thai population.

5.5 Statistical Population Comparison

5.5.1 Comparison of contemporary Thai and Black South African (Bidmos and Asala, 2005) descriptive statistics

5.5.2 Comparison of contemporary Thai and White South African Bidmos,2006) descriptive statistics

5.5.3 Comparison of contemporary Thai and Black American (Holland, 1995) descriptive statistics.

CHAPTER 6: CONCLUSIONS

REFERENCES

APPENDIX A: CHIANG MAI SKELETAL COLLECTION RAW DATA

\section{APPENDIX B: EXAMPLES OF CALCANEAL AND TALAR PATHOLOGIES}

Appendix B1: Example \#1 of pathological left calcaneus and left talus, excluded from analyses. (Catalogue number: 5407126) (Photo by: Shelby Scott) 235

Appendix B2: Example \#2 of pathological left calcaneus and left talus, excluded from analyses. (Catalogue number: 79-52) (Photo by: Shelby Scott)

Appendix B3: Example of degraded left calcaneus and left talus, excluded from analyses. (Catalogue number: 271-56) (Photo by: Shelby Scott)

Appendix B4: Example of degraded left calcaneus, excluded from analyses. (Catalogue number: 5301017) (Photo by: Shelby Scott)

APPENDIX C: NORMALITY TESTS

Appendix C1: Normality probability plot for male MAXL (Maximum Length) calcaneal measurements. 
Appendix C2: Normality probability plot for female MAXL (Maximum

Length) calcaneal measurements.

Appendix C3: Normality probability plot for male MAXH (Maximum Height)

calcaneal measurements

Appendix C4: Normality probability plot for female MAXH (Maximum

Height) calcaneal measurements

Appendix C5: Normality probability plot for male CFH (Cuboidal Facet

Height) calcaneal measurements.

Appendix C6: Normality probability plot for female CFH (Cuboidal Facet

Height) calcaneal measurements.

Appendix C7: Normality probability plot for male BH (Body Height)

calcaneal measurements

Appendix C8: Normality probability plot for female BH (Body Height)

calcaneal measurements

Appendix C9: Normality probability plot for male MINB (Minimum Breadth)

calcaneal measurements

Appendix C10: Normality probability plot for female MINB (Minimum

Breadth) calcaneal measurements

Appendix C11: Normality probability plot for male LAL (Load Arm Length)

calcaneal measurements

Appendix C12: Normality probability plot for female LAL (Load Arm

Length) calcaneal measurements

Appendix C13: Normality probability plot for male MIDB (Middle Breadth) calcaneal measurements

Appendix C14: Normality probability plot for female MIDB (Middle Breadth)

calcaneal measurements

Appendix C15: Normality probability plot for male DAFB (Dorsal Articular Facet Breadth) calcaneal measurements.

Appendix C16: Normality probability plot for female DAFB (Dorsal Articular

Facet Breadth) calcaneal measurements.

Appendix C17: Normality probability plot for male DAFL (Dorsal Articular Facet Length) calcaneal measurements ....

Appendix C18: Normality probability plot for female DAFL (Dorsal Articular Facet Length) calcaneal measurements 248

Appendix C19: Normality probability plot for male MTAL (Maximum Length of the Talus) talar measurements 
Appendix C20: Normality probability plot for male MTAL (Maximum Length of the Talus) talar measurements

\section{APPENDIX D: ACCURACIES OF OTHER POPULATIONS SIMPLE AND MULTIPLE REGRESSION EQUATIONS WHEN APPLIED TO THE THAI POPULATION}

Appendix D1: Simple scatterplot showing the correlation between documented living stature and the predicted living stature, using the male Black South African (Bidmos and Asala, 2005) MAXL simple regression equation.

Appendix D2: Simple scatterplot showing the correlation between documented living stature and the predicted living stature, using the female Black South African (Bidmos and Asala, 2005) MAXL simple regression equation.

Appendix D3: Simple scatterplot showing the correlation between documented living stature and the predicted living stature, using the male Black South African (Bidmos and Asala, 2005) MAXH simple regression equation.

Appendix D4: Simple scatterplot showing the correlation between documented living stature and the predicted living stature, using the female Black South African (Bidmos and Asala, 2005) MAXH simple regression equation.

Appendix D5: Simple scatterplot showing the correlation between documented living stature and the predicted living stature, using the male Black South African (Bidmos and Asala, 2005) BH simple regression equation.

Appendix D6: Simple scatterplot showing the correlation between documented living stature and the predicted living stature, using the female Black South African (Bidmos and Asala, 2005) BH simple regression equation.

Appendix D7: Simple scatterplot showing the correlation between documented living stature and the predicted living stature, using the male Black South African (Bidmos and Asala, 2005) MINB simple regression equation. .254

Appendix D8: Simple scatterplot showing the correlation between documented living stature and the predicted living stature, using the female Black South African (Bidmos and Asala, 2005) MINB simple regression equation. .254

Appendix D9: Simple scatterplot showing the correlation between documented living stature and the predicted living stature, using the male Black South African (Bidmos and Asala, 2005) LAL simple regression equation. 255

Appendix D10: Simple scatterplot showing the correlation between documented living stature and the predicted living stature, using the female Black South African (Bidmos and Asala, 2005) LAL simple regression equation. 255

Appendix D11: Simple scatterplot showing the correlation between documented living stature and the predicted living stature, using the male 
Black South African (Bidmos and Asala, 2005) MIDB simple regression equation.

Appendix D12: Simple scatterplot showing the correlation between documented living stature and the predicted living stature, using the female Black South African (Bidmos and Asala, 2005) MIDB simple regression equation.

Appendix D13: Simple scatterplot showing the correlation between documented living stature and the predicted living stature, using the male Black South African (Bidmos and Asala, 2005) DAFB simple regression equation.

Appendix D14: Simple scatterplot showing the correlation between documented living stature and the predicted living stature, using the female Black South African (Bidmos and Asala, 2005) DAFB simple regression equation.

Appendix D15: Simple scatterplot showing the correlation between documented living stature and the predicted living stature, using the male Black South African (Bidmos and Asala, 2005) DAFL simple regression equation. 258

Appendix D16: Simple scatterplot showing the correlation between documented living stature and the predicted living stature, using the female Black South African (Bidmos and Asala, 2005) DAFL simple regression equation.

Appendix D17: Simple scatterplot showing the correlation between documented living stature and the predicted living stature, using the male Black South African (Bidmos and Asala, 2005) multiple regression equation 1 (MAXL, MAXH, MIDB, and DAFB).

Appendix D19: Simple scatterplot showing the correlation between documented living stature and the predicted living stature, using the male Black South African (Bidmos and Asala, 2005) multiple regression equation 2 (MAXL, MAXH, and MIDB). 260

Appendix D20: Simple scatterplot showing the correlation between documented living stature and the predicted living stature, using the female Black South African (Bidmos and Asala, 2005) multiple regression equation 2 (MAXL, LAL, and DAFL). 260

Appendix D21: Simple scatterplot showing the correlation between documented living stature and the predicted living stature, using the male Black South African (Bidmos and Asala, 2005) multiple regression equation 3 (MAXL and MIDB).

Appendix D22: Simple scatterplot showing the correlation between documented living stature and the predicted living stature, using the female 
Black South African (Bidmos and Asala, 2005) multiple regression equation 3 (MAXL, MAXH, and DAFL).

Appendix D23: Simple scatterplot showing the correlation between documented living stature and the predicted living stature, using the male Black South African (Bidmos and Asala, 2005) multiple regression equation 4 (MAXL and MAXH).

Appendix D24: Simple scatterplot showing the correlation between documented living stature and the predicted living stature, using the male Black South African (Bidmos and Asala, 2005) multiple regression equation 4 (MAXH and DAFL).

Appendix D25: Simple scatterplot showing the correlation between documented living stature and the predicted living stature, using the male Black American (Holland, 1995) MTAL simple regression equation.

Appendix D26: Simple scatterplot showing the correlation between documented living stature and the predicted living stature, using the female Black American (Holland, 1995) MTAL simple regression equation.

APPENDIX E: ACCURACIES OF CROSS-VALIDATION 264

Appendix E1: Simple scatterplot illustrating the correlation between documented and predicted living stature, in a Thai population, using the male MAXL calcaneal simple regression equation.

Appendix E2: Simple scatterplot illustrating the correlation between documented and predicted living stature, in a Thai population, using the female MAXL calcaneal simple regression equation.

Appendix E3: Simple scatterplot illustrating the correlation between documented and predicted living stature, in a Thai population, using the male MAXH calcaneal simple regression equation. 266

Appendix E4: Simple scatterplot illustrating the correlation between documented and predicted living stature, in a Thai population, using the female MAXH calcaneal simple regression equation. .266

Appendix E5: Simple scatterplot illustrating the correlation between documented and predicted living stature, in a Thai population, using the male $\mathrm{CFH}$ calcaneal simple regression equation.

Appendix E6: Simple scatterplot illustrating the correlation between documented and predicted living stature, in a Thai population, using the male $\mathrm{BH}$ calcaneal simple regression equation. .268

Appendix E7: Simple scatterplot illustrating the correlation between documented and predicted living stature, in a Thai population, using the female $\mathrm{BH}$ calcaneal simple regression equation. .268 
Appendix E8: Simple scatterplot illustrating the correlation between documented and predicted living stature, in a Thai population, using the male MINB calcaneal simple regression equation.

Appendix E9: Simple scatterplot illustrating the correlation between documented and predicted living stature, in a Thai population, using the male LAL calcaneal simple regression equation.

Appendix E10: Simple scatterplot illustrating the correlation between documented and predicted living stature, in a Thai population, using the female LAL calcaneal simple regression equation

Appendix E11: Simple scatterplot illustrating the correlation between documented and predicted living stature, in a Thai population, using the male MIDB calcaneal simple regression equation.

Appendix E12: Simple scatterplot illustrating the correlation between documented and predicted living stature, in a Thai population, using the female MIDB calcaneal simple regression equation.

Appendix E12: Simple scatterplot illustrating the correlation between documented and predicted living stature, in a Thai population, using the female DAFB calcaneal simple regression equation.

Appendix E13: Simple scatterplot illustrating the correlation between documented and predicted living stature, in a Thai population, using the female DAFL calcaneal simple regression equation.

Appendix E14: Simple scatterplot illustrating the correlation between documented and predicted living stature, in a Thai population, using the male MTAL talar simple regression equation.

Appendix E15: Simple scatterplot illustrating the correlation between documented and predicted living stature, in a Thai population, using the female MTAL talar simple regression equation....

Appendix E16: Simple scatterplot illustrating the correlation between documented and predicted living stature, in a Thai population, using the male direct multiple regression equation 1 .

Appendix E17: Simple scatterplot illustrating the correlation between documented and predicted living stature, in a Thai population, using the female direct multiple regression equation 1.

Appendix E18: Simple scatterplot illustrating the correlation between documented and predicted living stature, in a Thai population, using the male direct multiple regression equation 2 .

Appendix E19: Simple scatterplot illustrating the correlation between documented and predicted living stature, in a Thai population, using the female direct multiple regression equation 2 . 
Appendix E20: Simple scatterplot illustrating the correlation between documented and predicted living stature, in a Thai population, using the male direct multiple regression equation 3

Appendix E21: Simple scatterplot illustrating the correlation between documented and predicted living stature, in a Thai population, using the female direct multiple regression equation 3 .

Appendix E22: Simple scatterplot illustrating the correlation between documented and predicted living stature, in a Thai population, using the male direct multiple regression equation 4 .

Appendix E23: Simple scatterplot illustrating the correlation between documented and predicted living stature, in a Thai population, using the female direct multiple regression equation 4 .

Appendix E24: Simple scatterplot illustrating the correlation between documented and predicted living stature, in a Thai population, using the male stepwise multiple regression equation 5 .

Appendix E25: Simple scatterplot illustrating the correlation between documented and predicted living stature, in a Thai population, using the female stepwise multiple regression equation 5 . 


\section{LIST OF TABLES}

Table 3.1 Sample size distribution for the Thai sample utilized in this research study.

Table 3.2 Measurement variable descriptions of the calcaneus .61

Table 3.3 Measurement variable description of the talus

Table 3.4 Regression equations (in $\mathrm{cm}$ ) for estimated skeletal height from the calcaneus of Black South Africans (Bidmos and Asala, 2005.

Table 3.5 Regression equations (in $\mathrm{cm}$ ) for estimated living stature from the talus of Black Americans (Holland, 1995).

Table 4.1 Normality probability p-values assessed in males and females.

Table 4.2 Descriptive statistics for calcaneal and talar measurement variables for both males and females.

Table 4.3 Two-sample t-tests evaluating sexual dimorphism of males and females for each calcaneal and talar measurement variable

Table 4.4 Statistical analyses of the intra-observer error rates and concordance correlation coefficients of reproducibility $(\mathrm{Rc})$ for the calcaneus and talus of individuals in the Chiang Mai skeletal collection.

Table 4.5 Statistical analyses of the inter-observer error rates and concordance correlation coefficients of reproducibility (Rc) for the calcaneus and talus of individuals in the Chiang Mai skeletal collection.

Table 4.6 Accuracies of the male Black South African (Bidmos and Asala, 2005) regression equations for the calcaneus when applied to the Thai population.

Table 4.7 Accuracies of the female Black South African (Bidmos and Asala, 2005) regression equations for the calcaneus when applied to the Thai population.

Table 4.8 Accuracies of the male and female Black American (Holland, 1995) regression equations for the talus when applied to the Thai population.... 
Table 4.9 Calcaneal and talar simple regression equations for male and female living stature estimation (in $\mathrm{cm}$ ) in a Thai population

Table 4.10 Direct and stepwise multiple regression equations for male and female living stature estimation (in $\mathrm{cm}$ ).

Table 4.11 Cross validation results of calcaneal and talar simple regression equations for the Thai population.

Table 4.12 Cross validation results of calcaneal and talar multiple regression equations for the Thai population.

Table 4.13 Comparison of male Thai mean measurements to a Black South African (Bidmos and Asala, 2005), White South African (Bidmos, 2006), and Black American (Holland, 1995) population.

Table 4.14 Comparison of female Thai mean measurements to a Black South African (Bidmos and Asala, 2005), White South African (Bidmos, 2005), and Black American (Holland, 1995) population.

Table 4.15 Comparison of male and female Thai measurements of the calcaneus to a Black South African population (Bidmos and Asala, 2005).

Table 4.16 Comparison of male and female Thai measurements of the calcaneus to a White South African population (Bidmos, 2006).

Table 4.17 Comparison of male and female Thai measurements of the talus to a Black American population (Holland, 1995).

Table 5.1 Comparison of the mean difference between the male maximum length of the calcaneus (MAXL) measurement variable and the female maximum length of the calcaneus (MAXL) measurement variable between different studies.

Table 5.2 Comparison of the mean difference between the male maximum length of the talus (MTAL) measurement variable and the female maximum length of the talus (MTAL) measurement variable between different studies. 138

Table 5.3 Comparison of Standard Error of the Estimate (SEE) ranges for the current research study and previous studies by different authors. 


\section{LIST OF FIGURES}

Figure 2.1 Dorsal (superior) view of the left human foot depicting the tarsal bones. (Photo by Shelby Scott)

Figure 2.2 Medial view of a typical left calcaneus and typical left talus depicting the articulation between the calcaneus and the talus. (Photo by Shelby

Scott)

Figure 2.3 Dorsal (superior) view of a typical left calcaneus. (Photo by Shelby

Scott)

Figure 2.4 Plantar (inferior) view of a typical left calcaneus. (Photo by Shelby Scott)

Figure 2.5 Medial view of the left human foot depicting the articulation between the calcaneus and the talus to form the subtalar joint. (Photo by Shelby Scott)

Figure 2.6 Dorsal (superior) view of a typical left talus. (Photo by Shelby Scott) .25

Figure 2.7 Posterolateral view of the left human foot depicting the articulation between the talus, the tibia, and the fibula to form the talocrural (ankle) joint. (Photo by Shelby Scott) .26

Figure 3.1 Distribution of age-at-death in the Thai skeletal sample utilized for this research study

Figure 3.2 Medial view of a typical left calcaneus depicting calcaneal measurement variables: Maximum Length (MAXL), Maximum Height (MAXH), Cuboidal Facet Height (CFH), Body Height (BH), and Load Arm Length (LAL). (Photo by Shelby Scott)

Figure 3.3 Superior view of a typical left calcaneus depicting calcaneal measurement variables: Minimum Breadth (MINB), Middle Breadth (MIDB), Dorsal Articular Facet Breadth (DAFB), and Dorsal Articular Facet Length (DAFL). (Photo by Shelby Scott). 
Figure 3.4 Superior view of a typical left talus depicting the Maximum Length of the Talus (MTAL) measurement variable. (Photo by Shelby Scott)

Figure 3.5 Mastercraft electronic (Vernier) caliper used for skeletal measurements. (Photo by Shelby Scott)

Figure 4.1 Normality probability plot for the male Maximum Length (MAXL) calcaneal measurement variable.

Figure 4.2 Simple scatterplot illustrating the correlation between documented and predicted living stature in the Thai male population using the male Black South African Maximum Length (MAXL) simple regression equation for the calcaneus (Bidmos and Asala, 2005).

Figure 4.3 Simple scatterplot illustrating the correlation between documented and predicted living stature in the Thai male population using the male Black American (Holland, 1995) Maximum Length of the Talus (MTAL) simple regression equation 100

Figure 4.4 Simple scatterplot illustrating the correlation between documented and predicted living stature, in a Thai population, using the Maximum Length (MAXL) calcaneal simple regression equation. 116 


\section{CHAPTER 1: INTRODUCTION}

\subsection{Research Objectives}

Skeletonized remains are commonly recovered within medico-legal contexts and mass disasters, requiring careful examination by a forensic anthropologist. Stature is an integral component of the biological profile, which examines characteristics such as sex, age at death, ancestry, and stature. Countries that have endured mass disasters, either natural (e.g. earth quakes, hurricanes, and tsunamis), or non-natural (e.g. terrorist activities, wars, or political crisis), have stressed the importance of including forensic anthropologists as part of the interdisciplinary teams which assist with disaster victim identification (Prinz et al., 2007; Traithepchanapai et al., 2016). In Thailand, the 2004 tsunami disaster highlighted the lack of forensic anthropology expertise in the country and the lack of population-specific methods in the region (Traithepchanapai et al., 2016). The objectives of this research are to:

1. investigate whether regression equations for the calcaneus, which were developed on a Black South African population (Bidmos and Asala, 2005), and regression equations for the talus, which were developed on a Black American population (Holland, 1995), can accurately predict the living stature of individuals from a contemporary Thai population.

2. develop population-specific simple regression and multiple regression equations for the calcaneus and the talus of the contemporary Thai population should the Black South African (Bidmos and Asala, 2005) and Black American (Holland, 1995) equations not accurately predict the living stature of the Thai individuals.

3. compare the results from the contemporary Thai population to other populations. 


\subsection{Potential for Stature Estimation from the Calcaneus and the Talus}

The estimation of stature is a critical factor in the identification of unknown skeletal remains, providing one aspect of an individual's physiognomy. Most bones in the human body have been assessed for their potential in estimating stature. Long bones, such as the femur and the tibia, are preferred for stature estimation (Trotter and Glesner, 1952). In mass disaster and forensic contexts however, it is likely that these bones will be found fragmented due to postmortem damage and taphonomic changes. Previous researchers have used fragmentary long bones to derive regression equations for the estimation of stature (Simmons et al., 1990; Steele et al., 1969). However, accuracy rates are enhanced when stature is estimated from complete bones rather than fragmentary remains (Smith, 1996). Therefore, in order to ensure the most accurate estimation of stature from unknown human remains, it is necessary to develop methods of stature estimation from preservationally favoured and/or complete skeletal elements.

Previous research has cited stature estimation methods from less frequently analyzed complete skeletal elements such as the skull (Chiba and Terazawa, 1998; Fully, 1956; González-Colmenares et al., 2016; Introna et al., 1993; Krishan, 2008; Patil and Mody, 2005; Torimitsu et al., 2016), the sternum (Dwight, 1980; Marinho et al., 2012; Menezes et al., 2009, 2011; Singh et al., 2011; Zhang et al., 2015), the clavicle (Balvir et al., 2012; Jit and Singh, 1956; Rani et al., 2011; Singh and Sohal, 1952), the scapula (Campobasso et al., 1998; Giurazza et al., 2013; Shulin and Fangwu, 1983; Torimitsu et al., 2015; Zhang et al., 2016), the metacarpals (Khanpetch et al., 2012; Kimura, 1992; Meadows and Jantz, 1992; Musgrave and Harneja, 1978; Wilbur, 1998; Zaher et al., 2011), the hand phalanges (Habib and Kamal, 2010; Jasuja and Singh, 2004; Jee and Yun, 
2015; Shintaku and Furuya, 1990), the vertebrae (Jason and Taylor, 1995; Nagesh and Kumar, 2006; Rodríguez et al., 2016; Terasawa et al., 1990; Tibbetts, 1981; Torimitsu et al., 2015), the sacrum (Fully, 1956; Giroux and Wescott, 2008; Karakas et al., 2011; Pelin et al., 2005; Pininski and Brits, 2014; Torimitsu et al., 2014), and the metatarsals (Bidmos, 2008; Byers et al., 1989; De Groote and Humphrey, 2011). However, there has been minimal research using osteometric criteria of individual tarsals, or bones of the foot, for stature estimation (Bidmos, 2006; Bidmos and Asala, 2005; Fully, 1956; Holland, 1995; Pablos et al., 2013).

The calcaneus and the talus, in particular, are underrepresented in the forensic and anthropological literature for stature estimation. Yet, these bones have been shown to be very accurate predictors of stature estimation. The anatomical method of stature reconstruction, first described by Fully (1956) and revised by Raxter et al. (2006), demonstrated the usefulness of the calcaneus and the talus for stature estimation, with $95 \%$ of individuals estimated correctly to within $4.5 \mathrm{~cm}$. More recently, regression equations for the estimation of stature using measurements of the calcaneus and the talus have been generated for Black American, White American, Black South African, and South African populations (Bidmos, 2006; Bidmos and Asala, 2005; Holland, 1995; Pablos et al., 2013) with success. To date, these are the only studies employed for stature estimation using osteometric criteria of the calcaneus and the talus.

In the human body, the calcaneus and the talus are the largest tarsal bones and have been shown to produce high accuracy rates for human identification as they are often well preserved and able to withstand high tensile forces (Bidmos, 2006; Bidmos and Asala, 2005; Hall and Shereff, 1993). This increased preservation is related to the increased strength and density of the bones trabeculae and the smaller surface area of the 
calcaneus and the talus. Small, compact bones, such as the calcaneus and the talus, have a greater chance of being recovered intact as the bones smaller surface area expose less bone to destructive taphonomic elements (Bidmos and Asala, 2005). The calcaneus and the talus are often encased in socks and/or shoes, which serve as protection for the feet (Peckmann et al., 2015; Pickering, 1986; Saul and Saul, 2005). Furthermore, the unique morphology of the calcaneus and the talus allow for rapid and accurate anatomical identification during excavation (Lee et al., 2012; Susman, 1983). These characteristics make the calcaneus and the talus ideal for the estimation of stature and identification of unknown human remains.

\subsection{Concepts of Identity and Terminology in Forensic Anthropology}

\subsubsection{Differences between Sex and Gender}

The terms sex and gender are different concepts within anthropology (Little and McGivern, 2014). An individual's biological sex (i.e. sex) refers to the biological and physiological division into male and female at the time of conception, but may not correlate with the gender to which an individual identifies (Armelagos, 1998; Steckley and Kirby Letts, 2013).

An individual's sex can be consistently determined and accurately classified during life based on the existence of both genetic and morphological differences between males and females. Sex chromosomes are structurally distinct between males and females. Females carry two X chromosomes (XX) and males carry one $\mathrm{X}$ chromosome and one Y chromosome (XY (Charlesworth, 1991; Jacobs and Strong, 1959). The Y chromosome controls male development. The testis-determining factor (TDF) is found on 
the $\mathrm{Y}$ chromosome and is responsible for the initiation of male sex determination in humans (Graves, 1995). Subsequent steps in male phenotype establishment are regulated by testicular hormones independent of the $\mathrm{Y}$ chromosome and other genes carried through the $\mathrm{Y}$ chromosome. Genes carried through the $\mathrm{Y}$ chromosome are expressed in the testis, which influence spermatogenesis, or the production and development of mature spermatozoa (Graves, 1995).

Sex hormones influence skeletal morphology and contribute to the sexually dimorphic differences observed in male and female skeletal anatomy (Kottak, 2011). Overall, female skeletal elements are smaller and less robust than male skeletal elements. Relative to female crania, male crania typically display: more prominent supraorbital ridges, a more prominent glabellar region, heavier temporal and nuchal lines, less frontal and parietal bossing, broader palates, squarer eye orbits, more rugose muscle attachments, and larger mastoid processes, sinuses, and occipital condyles (Buikstra and Ubelaker, 1994; Graw et al., 1999; Meindl et al., 1985). There are also functional differences between male and female pelvic morphology as a result of parturition, or the process of childbirth; female pelvic inlets are relatively wider than those of males with wider greater sciatic notches, elongated pubic portions of the os coxae, a larger subpubic angle, a more distinct preauricular sulcus, and a smaller acetabulum (Buikstra and Ubelaker, 1994; Phenice, 1969; Rogers and Saunders, 1994). The manifestation of these sexually dimorphic differences in the skeletal anatomy allow forensic anthropologists to estimate the sex of an individual based on biological features.

Gender refers to the roles, behaviours, and characteristics associated with biological males and females (Konigsberg et al., 1998). Concepts of gender are socially constructed and may vary between cultures, communities, and social classes (Kottak, 
2011; Risman, 2004). Thailand, for example, has a three-gender system (i.e. male, female, and a third gender category called kathoey), which is best represented as a continuum with permeable boundaries that allow for the flow and co-existence of multiple gender identities (Ocha, 2012). As such, gender is unable to be estimated through the analysis of skeletal remains and therefore this terminology will not be used in the current research study.

An individual's sex may not be reflective of the gender to which that individual associates. As the term sex reflects biological differences in skeletal anatomy, and the term gender reflects a social construct, the term sex will be used to differentiate between male and female skeletal remains in the current research study.

\subsubsection{The Origin and Construction of 'Race' in Physical Anthropology}

The term 'race' emerged between the sixteenth and eighteenth centuries and became synonymous with words such as, 'sort', 'kind', 'type', 'breed', and 'species' (Allen, 1994, 1997; Hannaford, 1996; Smedley, 1998). Carl Linnaeus, an eighteenthcentury botanist, was the first scientist to categorize humans into groups based on observable features: Europeaus (albus) or "white", Americanis (rufescens) or "red", Asiaticus (fuscus) or "dark", and Africanus (niger) or "black" (Harrison, 2010). Johan Blumenbach continued this categorization in 1779, classifying human "varieties" into the following 'races': the Caucasian or European "white" 'race' the Mongolian or "yellow" 'race' (including all East Asians and some Central Asians), the Malayan or "brown" 'race' (including Southeast Asians and Pacific Islanders), the Ethiopian or "black” 'race' (including sub-Saharan Africans), and the American or "red" 'race' (including American Indians) (Blumenbach, 1865:56,209). By the late eighteenth century, the concept of 'race' 
had solidified as a reflection of social hierarchy based on the physical and observable differences of populations (Smedley, 1998; Smedley and Smedley, 2005). The emergence of 'race', as a social classification, arose from the ethnocentric European notions of superiority towards Indigenous (or Non-European) populations that were deemed to be savage, uncivilized, and inferior in comparison (Harrison, 2010; Prins, 2002; Smedley, 1998; Smedley and Smedley, 2005; Winant, 2000). This 'race' ideology developed in conjunction with the legal establishment of African slave labour and the conquest of North American Indigenous populations, thus rationalizing inequalities such as slavery, colonialism, eugenics, and segregation (Mukhopadhyay and Mosese, 1997; Smedley, 1998).

In the nineteenth century, scientists and anthropologists contributed to the perpetuation of 'race' and the notion of social hierarchies based on observable 'racial' features. Sociocultural anthropologists, such as L.H Morgan and E.B. Tylor, worked with physical anthropologists to rank human 'races' along an evolutionary path from 'savage' to 'civilized' using physical indicators and craniometric data (Mukhopadhyay and Mosese, 1997). Craniometry was used throughout the nineteenth century as a way of supporting the notion of 'race' superiority through the study of cranial size and weight to infer the mental capacity of various 'racial' groups (Mukhopadhyay and Mosese, 1997; Smay and Armelagos, 2000).

Twentieth century anthropologists helped expose the inaccuracies surrounding the biological concept of 'race'. In the early twentieth century, the cephalic index was utilized as a way of measuring evolutionary advancement. Franz Boas discredited craniometry and the hypothesis of a link between mental capacity and 'race' by demonstrating that cranial size and shape was dependent on environmental factors (Boas, 
1912). In 1942, Ashley Montagu argued that external physical traits are not the cause of human variation, an assertion that further inspired abandonment of the 'race' concept (Montagu, 1997). The development of population genetics, in the mid-twentieth century, provided further evidence against the biological concept of 'race'. Population geneticists focused on how evolutionary processes such as genetic drift, migration, mutation, and natural selection could affect inherited biological traits (Mukhopadhyay and Mosese, 1997). By 1972, population geneticist Richard Lewontin had demonstrated that more genetic variation exists within, rather than between, the major 'racial' groups, thus demonstrating that 'race' has no genetic or taxonomic significance (Lewontin, 1972: 397; Mukhopadhyay and Mosese, 1997).

While most anthropologists now reject the existence of the traditional 'race' concept, the classification of individuals into groups persists in physical and forensic anthropology (Hunt and Megyesi, 2008; Kennedy, 1995; Ousley et al., 2009; Sauer, 1992). When creating a biological profile, forensic anthropologists analyze variation in skeletal components in order to classify human remains into population groups (Albanese and Saunders, 2006; Ousley et al., 2009; Sauer, 1992; Smay and Armelagos, 2000). For example, morphological features of the cranium show strong geographical patterning and have been used to classify groups of individuals based on geographic origins (Ousley et al., 2009; Patriquin et al., 2002). Populations of Asian origins display crania with robust zygomatic bones, circular eye orbits, and an elliptic shaped palate; populations of European origins display crania with receding/small zygomatic bones, heavy glabellar and supraorbital regions, and a parabolic shaped palate; and populations of African origins display crania with pronounced facial and alveolar prognathism, rectangular eye 
orbits, and a hyperbolic shaped palate (Giles and Elliot, 1962; Gill, 1995; Gill and Rhine, 1990).

However, law enforcement members often use primary descriptors such as an individual's 'race' when compiling information on missing persons. The options available for categorization of missing persons may be limited; The National Centre for Missing Persons and Unidentified Remains (NCMPUR) of the Royal Canadian Mounted Police (RCMP) for example, provides the following descriptive options: “Aboriginal”, “Asian”, “Black”, "East Indian”, "White”, "Other”, or "Unknown” (NCMPUR, 2017). As forensic anthropologists function in cooperation with law enforcement, legal representatives, and members of the community or the jury, they must report their findings regarding unidentified human skeletal remains in terms meaningful to their audience; 'race' remains a primary descriptor for investigators working in law enforcement (Adams, 2007). As the term 'race' denotes no genetic or taxonomic relationships, and therefore will not be used in the current research study to define biological variation between human populations, manifested in skeletal morphology.

\subsubsection{Terminology: Ethnicity and Ancestry}

Ethnicity is defined as cultural distinctions between groups of individuals which share, for example, a common language, religion, history, system of beliefs or traditions, or geographical region (Smedley and Smedley, 2005:17). An individual's ethnicity, or associated ethnic group, is self-defined and flexible, or subject to change (Barth, 1998). Cultural traits are learned and are transmissible to others and, therefore, one may adopt the cultural traits of another ethnic group to become participants in that ethnicity (Smedley and Smedley, 2005). As ethnicity relates to a self-identifying cultural concept 
and does not describe genetic relationships or biological traits, the term ethnicity will not be used in the current research study to define biological variation between human populations, manifested in skeletal morphology.

Ancestry is defined as the grouping of individuals based on a shared genetic makeup (Bamshad, 2005:937). An individual's genetic makeup is determined by the geographic location from which they originated (Bamshad, 2005; Ember and Ember, 1988; Jorde and Wooding, 2004; Konigsberg et al, 2009). Individuals that originate from a specific geographic location will share a gene pool, or a collection of gene variants, and will display similarities in genetic allele frequencies and physical traits (Molnar, 1983; Witherspoon et al., 2007). Ancestry can thus be examined by a forensic anthropologist based on morphological skeletal traits, which are the result of microevolutionary processes and environmental stresses associated with geographic origin (Konigsberg et al., 2009). The term ancestry is based in biological traits and examines human genetic variation. Therefore, this term will be used in the current research study to define biological variation between human populations manifested in skeletal morphology.

\subsection{Forensic Anthropology and Disaster Victim Identification in Thailand}

The history, development, and practice of forensic anthropology in Thailand have been relatively limited (Kranioti and Paine, 2011; Traithepchanapai et al., 2016). The anatomist H.S Sankas (1930) was the first Thai individual to study and publish within the field of physical anthropology, conducting research on the relationship between cranial modules and cranial capacity at the United States National Museum. The first research, although unpublished, investigating the skeletal anatomy of Thai individuals is attributed 
to Dr. Sood Sangvichien at the Department of Anatomy, Faculty of Medicine, Siriraj Hospital, in Bangkok, Thailand (Traithepchanapai et al., 2016). Sangvichien examined morphological features of the preauricular sulcus for the estimation of sex within a Thai population (Sangvichien et al., 1950). Most early anthropological research in Thailand, including that of Sangvichien, remained unpublished, or was only published domestically (i.e. not in English), which resulted in limited representation of Thai research within international forensic and anthropological literature (Traithepchanapai et al., 2016).

The lack of forensic and anthropological expertise in Thailand was demonstrated during the 'Boxing Day tsunami' (Traithepchanapai et al., 2016). On 26 December 2004, an earthquake measuring 9.1 on the Richter scale occurred off the west coast of Sumatra, Indonesia, triggering a catastrophic tsunami (i.e. 'The Boxing Day tsunami') that was responsible for 5,395 deaths in Thailand (James, 2005; Sribanditmongkol et al, 2017; Wright et al., 2015). The magnitude of this disaster was beyond the expectations and experiences of the Thai organizations responsible for victim identification and highlighted the need for more efficient forensic and victim identification management strategies (Traithepchanapai et al., 2016).

This disaster demonstrated Thailand's inability to independently handle a mass disaster of this magnitude, and the necessity of having locally trained forensic specialists and experts in the victim identification process. International experts and non-profit organizations joined in the identification effort under a consolidated Thai Tsunami Victim Identification (TTVI) operation. The TTVI operation promoted international cooperation under Thai authority, and was formed to standardize the identification process using the disaster victim identification (DVI) protocol outlined by INTERPOL (INTERPOL, 2014; Sribanditmongkol et al, 2017; Wright et al., 2015). 
In the past, international anthropological involvement in the DVI process has been limited to the analysis of skeletal remains and subsequent establishment of a biological profile. More recently, however, the role of the forensic anthropologist in the DVI process has expanded to include the development and implementation of quality assurance and quality control, as well as greater participation at the disaster scene, in the morgue (particularly with initial triage), and with the reconciliation process (Blau and Briggs, 2011; Mundorff, 2012). Anthropological involvement in the DVI process in Thailand was limited; Kahana and Hiss (2009) stress that the DVI team in Thailand would have benefitted from the assistance of forensic anthropologists during the identification process.

To promote forensic anthropology and osteological research within Thailand, and thus enhance the DVI process in the region, the Forensic Osteology Research Center (FORC) in the Faculty of Medicine of Chiang Mai University, launched a doctoral program in forensic osteology in 2013. Researchers at the Forensic Osteology Research Center (FORC) created an osteological reference collection known as the Chiang Mai skeletal collection to assist in the development of population-specific methods of skeletal identification for the Thai population (Traithepchanapai et al., 2016). Despite these efforts, the development of forensic anthropology in Thailand remains limited in terms of training, professional opportunities, and representation within the forensic and anthropological literature (Traithepchanapai et al., 2016). The continuation of forensic anthropology research in Thailand is necessary in order to ensure population-specific methods are available for future tragic events. 


\subsection{Skeletal Collection: Forensic Osteology Research Center, Chiang Mai}

\section{$\underline{\text { University }}$}

The Chiang Mai skeletal collection is housed at the Forensic Osteology Research Center (FORC) in the Faculty of Medicine of the Chiang Mai University in Chiang Mai, Thailand. Skeletonization of individual remains for inclusion in the collection begins with dismemberment and maceration, or defleshing, followed by wrapping the bones in plastic mesh bags, and burial within a sand-filled container to allow for decomposition. Each burial is marked and left for four to six months or until no soft tissue remains on the bone. When the skeletal remains are recovered, they are kept in hydrogen peroxide for approximately three days before they are allowed to air dry. Once dry, each bone becomes labelled with its accession number and all the bones of each individual are stored in a singular, labelled plastic container. Metacarpals, carpals, metatarsals, tarsals, and phalanges are kept in labelled plastic bags (or are articulated with copper wire) to avoid confusion or loss, and are included within their respective container (King et al., 1998; Traithepchanapai et al, 2016).

The collection currently consists of 475 (291 males and 184 females) documented skeletons (i.e. known sex, age at death, occupation, cause of death, birth dates, and death dates) who donated their bodies for educational purposes. The donors were residents of Chiang Mai and adjacent villages and, therefore, this collection is representative of a northern Thai population (King et al., 1998; Traithepchanapai et al, 2016). Individuals represented in the Chiang Mai skeletal collection resided within the lower middle to middle socioeconomic range, with recorded occupations such as farmer, civil servant, teacher, or retiree (King et al., 1998; Traithepchanapai et al, 2016). Individuals used for 
the current research study have birth dates between 1914 and 1995 and death dates between 2006 and 2014. This skeletal collection was chosen for the current research study as it represents a contemporary population from Thailand; all of the individuals lived the majority of their life during the twentieth century. 


\section{CHAPTER 2: BACKGROUND}

\subsection{The Skeletal System and Anatomy of the Human Foot}

The skeletal system is the supporting framework of the body and is formed by bony and cartilaginous elements, which are articulated via connective tissue structures. The skeletal system also functions to protect internal organs from injury, assist in movement, maintain mineral homeostasis, produce blood cells in a process called hemopoiesis, and to store triglycerides, a potential chemical energy reserve (Faller et al, 2004:116; Tortora and Derrickson, 2014:170).

At the microscopic level, the structure of bone contains an extracellular matrix composed of $15 \%$ water, $30 \%$ collagen fibers, and $55 \%$ crystallized mineral salts. The most abundant mineral salt is calcium phosphate $\left[\mathrm{Ca}_{3}\left(\mathrm{PO}_{4}\right)_{2}\right]$, which combines with calcium hydroxide $\left[\mathrm{Ca}(\mathrm{OH})_{2}\right]$ to form hydroxyapatite crystals $\left[\mathrm{Ca}_{10}\left(\mathrm{PO}_{4}\right)_{6}(\mathrm{OH})_{2}\right]$. Additional mineral salts such as calcium carbonate, and ions such as magnesium and potassium, combine with the hydroxyapatite crystals as they are deposited in the collagen fibre matrix, crystallizing and hardening the tissue in a process known as calcification. While the crystallized mineral salts are responsible for the hardness of bone, the collagen fibres provide tensile strength, and are responsible for the bone's flexibility (Tortora and Derrickson, 2014:172).

Adult human bone is composed of two types of bone tissue. A solid, dense external layer known as compact or dense (cortical) bone functions to provide overall protection and to resist the stresses associated with weight and movement. An internal marrow cavity, comprised of spongy or cancellous (trabecular) bone, which resembles an open lattice structure, that functions to reduce the overall weight of the bone, allowing it 
to move more readily when pulled by skeletal musculature (Martini and Nath, 2009:188; Tortora and Derrickson, 2014:173).

The skeletal system can be divided into two principle divisions: the axial skeleton, consisting of bones surrounding the longitudinal axis of the body, and the appendicular skeleton, consisting of the bone of the upper and lower limbs and those which form the pelvic and shoulder girdles that connect the limbs to the axial skeleton (Tortora and Derrickson, 2014:193). The bones of the foot (i.e. the tarsals, the metatarsals, and the phalanges) are part of the appendicular skeleton and are arranged in two arches that are held in position by ligaments and tendons, which function to support and distribute the weight of the body (Hicks, 1954; Salathé et al., 1986). The foot consists of fourteen phalanges, five metatarsals, and seven tarsal bones including the calcaneus, talus, navicular, cuboid, and three cuneiform bones: medial (first) cuneiform, intermediate (second) cuneiform, and lateral (third) cuneiform (Figure 2.1). The calcaneus and the talus are important tarsal bones, owing to their role in locomotion and weight distribution (Figure 2.2). 


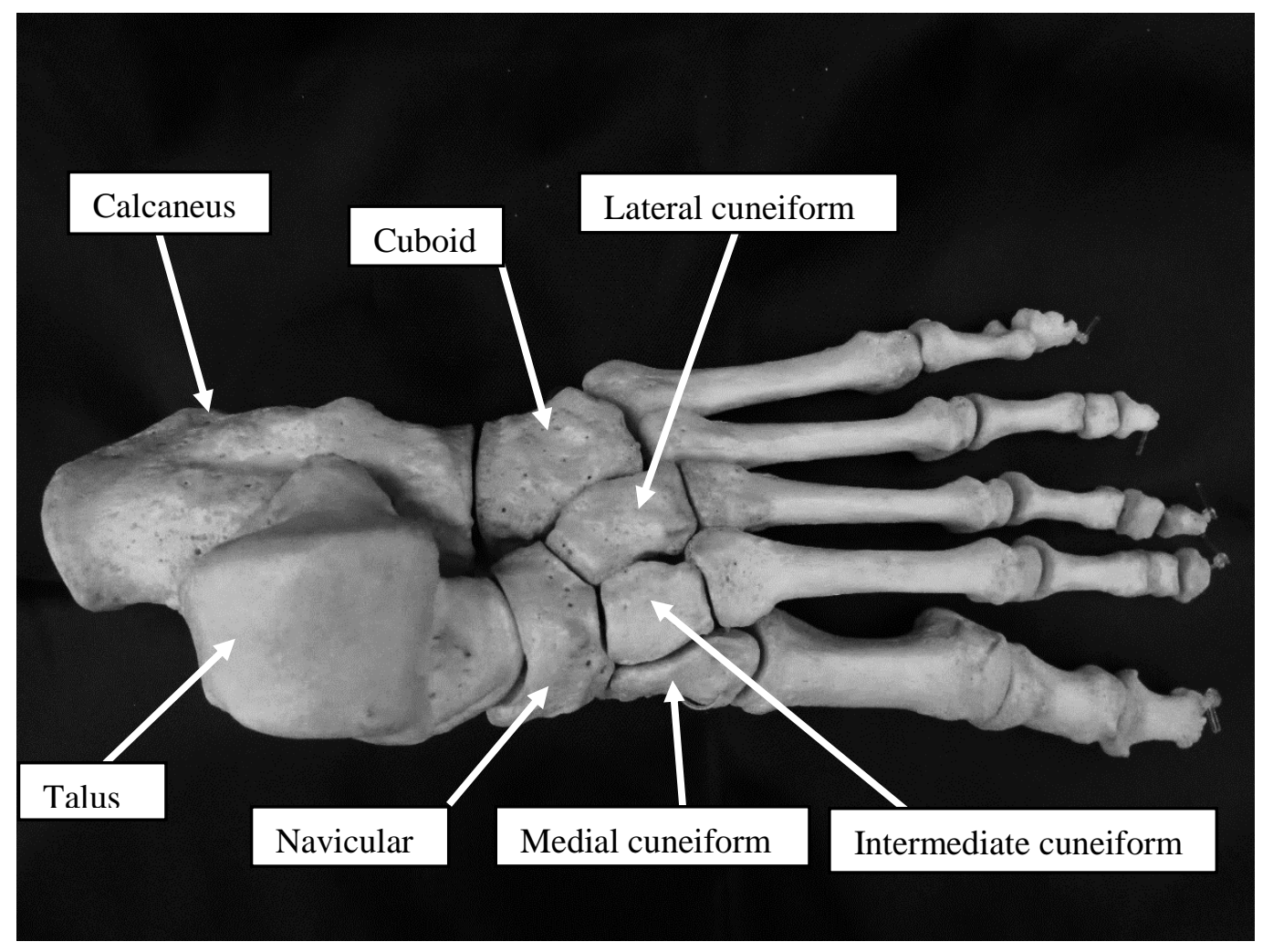

Figure 2.1 Dorsal (superior) view of the left human foot depicting the tarsal bones. (Photo by Shelby Scott)

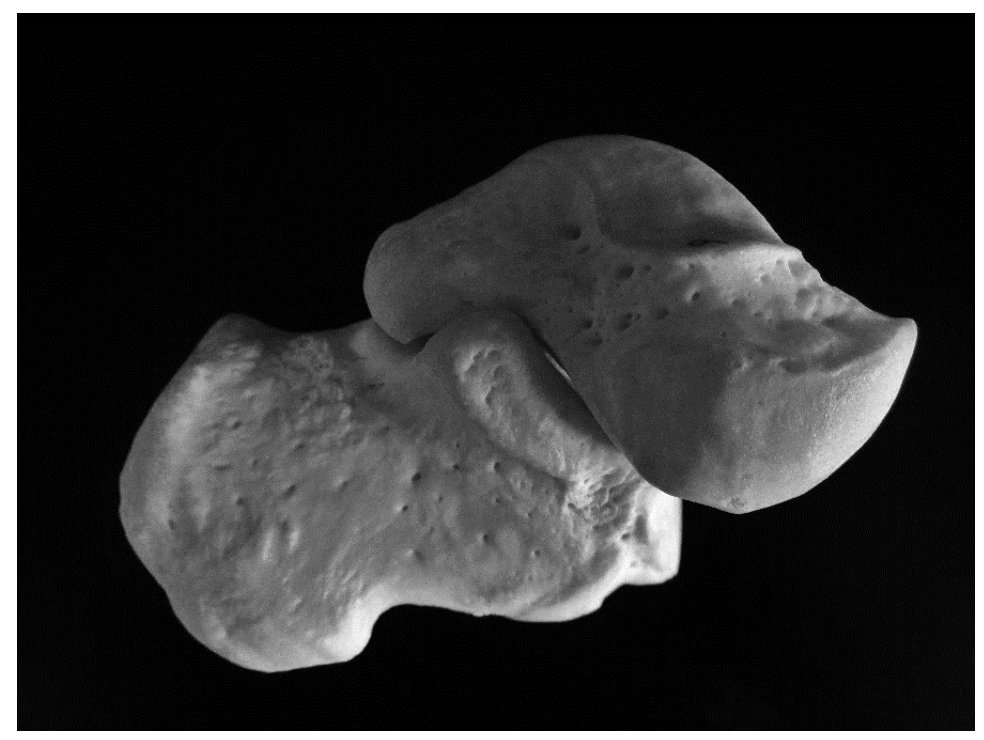

Figure 2.2 Medial view of a typical left calcaneus and typical left talus depicting the articulation between the calcaneus and the talus. (Photo by Shelby Scott) 


\subsubsection{Calcaneus and Talus: Growth and Development}

Postcranial skeletal tissue is formed from mesenchymal cells while connective tissue cells are derived from the mesoderm (the middle layer of cells formed in the early development of an embryo). Mesenchymal cells condense and form models of bone in areas where ossified tissue will ultimately form. Embryonic bone tissue development proceeds through two ossification processes: intramembranous ossification and endochondral ossification. Intramembranous ossification is the process by which bone tissue is formed directly within the mesenchyme, and is responsible for the creation of the skull and the vertebrae. Endochondral ossification is the process by which bone tissue is formed within hyaline cartilage that develops from the mesenchyme, and is responsible for the development of the upper and lower limbs (Tortora et al., 2014:253).

The skeleton of the lower limbs is derived from mesoderm and developed through endochondral ossification (Tortora et al., 2014:254). At approximately four weeks of prenatal development, a mesenchymal skeleton (i.e. a skeletal model consisting of mesenchyme) exists in the lower limb buds, which will eventually form the left and right legs. The first appearance of tarsal and metatarsal elements of the foot also occurs at four weeks of prenatal development, though tarsal elements are not fully developed at this time and the feet cannot be differentiated from the lower limb buds (Henke and Reyher, 1874; Leboucq, 1886; Meredith, 1944; Scheuer and Black, 2004; Straus, 1927; Viladot et al., 1984). By the sixth week of prenatal development, a cartilaginous skeleton has formed from the mesenchyme through endochondral ossification. At this time, the lower limb buds have developed into foot plates, distal segments of the lower limb buds that represent the beginning of the feet. By the eighth week of prenatal development, the 
lower limb buds have developed into their definitive postnatal form in which the thigh, the leg, and the foot are distinguishable from one another (Meredith, 1944; Straus, 1927; Viladot et al., 1984). Viladot et al. (1984) have noted that it is at approximately this time that modifications of the subtalar joint occur as the sustentaculum tali appears and the talus becomes positioned superior to the calcaneus, forming the ankle in its definitive form.

At the end of the eighth week of prenatal development, endochondral ossification of the lower limbs and tarsals has begun (Fritsch et al., 1996; Gardner et al., 1959; Tortora et al., 2014: 254). Studies have demonstrated that the calcaneus is the first of the tarsal bones to begin ossification (Gardner et al., 1959; Hasselwander, 1903; Hintzsche, 1930; Scheuer and Black, 2004). Pryor (1923) shows that ossification of the calcaneus occurs at approximately 16 weeks of prenatal development for females and at approximately 20 weeks of prenatal development for males. Though the cartilage of the talus is the first of the tarsal bones to demonstrate vascular invasion, ossification of the talus does not begin until approximately 22 to 29 weeks of prenatal development in females and 24 to 32 weeks of prenatal development in males (Gardner et al., 1959; Pryor, 1923).

\subsubsection{Calcaneus: Anatomy and Function}

The calcaneus, or "heel bone", is the largest tarsal bone of the foot, located inferior to the talus and articulating anteriorly with the cuboid. The calcaneus is comprised of several important and distinguishable structures: the calcaneal tuberosity, 
the lateral and medial processes, the peroneal tubercle, the sustentaculum tali, the posterior facet, and the sustentacular sulcus (Figures 2.3, 2.4).

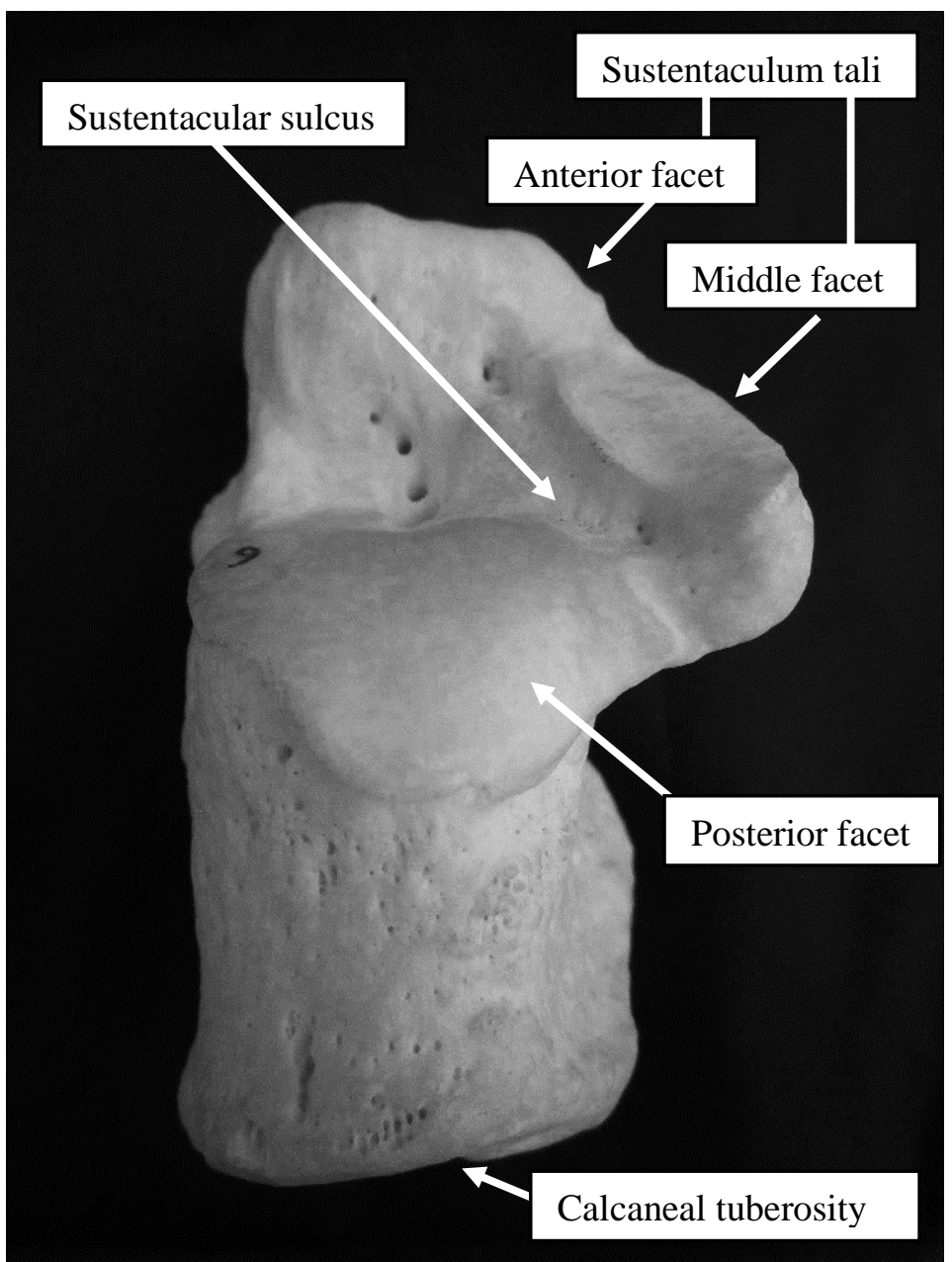

Figure 2.3 Dorsal (superior) view of a typical left calcaneus. (Photo by Shelby Scott) 


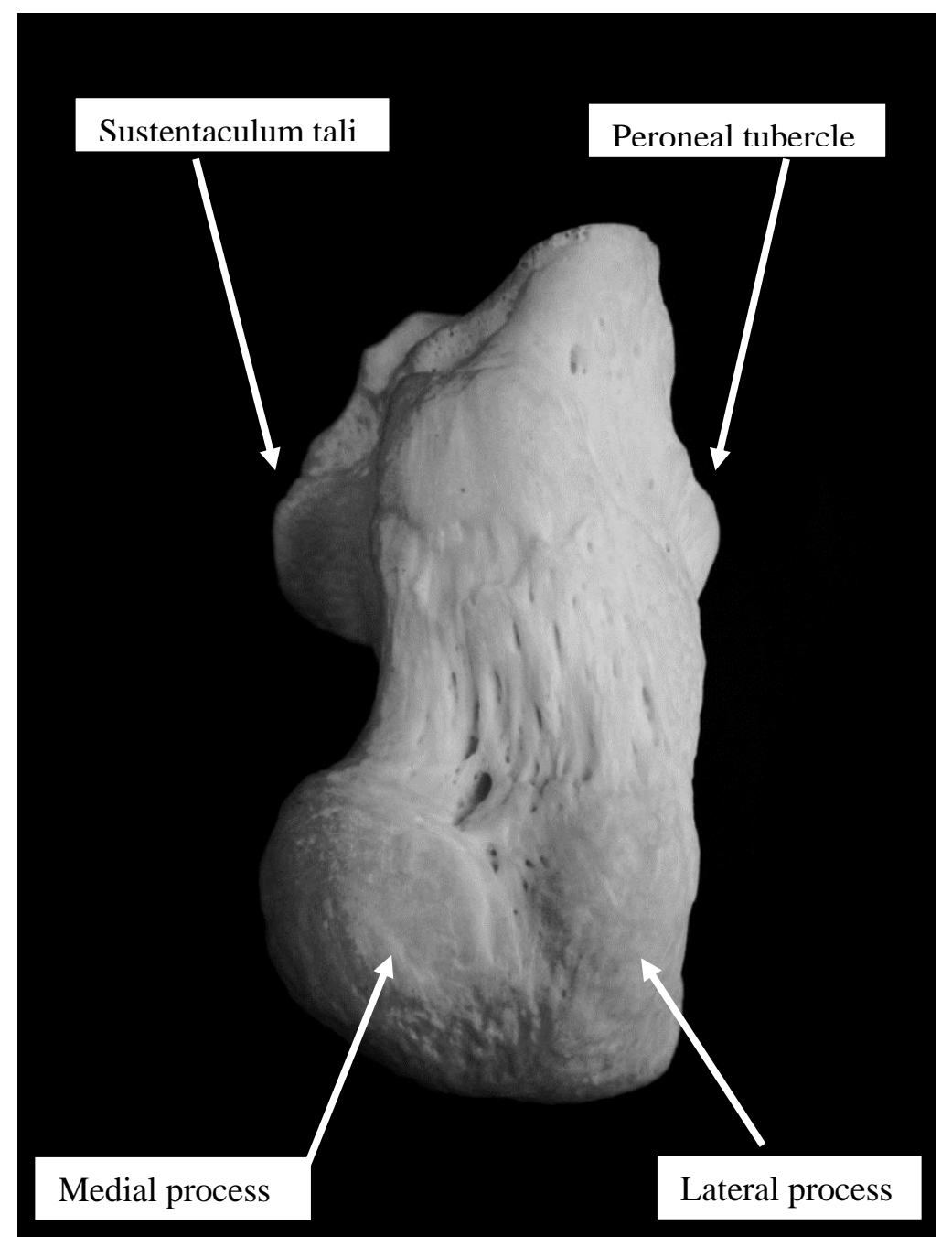

Figure 2.4 Plantar (inferior) view of a typical left calcaneus. (Photo by Shelby Scott)

The calcaneal tuberosity is the large, blunt, nonarticular, posterior process of the foot and is the insertion point for the calcaneal (Achilles) tendon (Doral et al., 2010; Keener and Sizensky, 2005). Tendons are responsible for the transmission of force from muscle to bone (Tai and Williams, 2007). The calcaneal insertion of the Achilles tendon is designed to aid the dissipation of stress from the tendon to the calcaneus, thus offering a mechanical advantage during locomotion (Doral et al., 2010). The lateral and medial processes of the calcaneal tuberosity are located on the inferior (plantar) aspect, and 
function to anchor several intrinsic muscles of the foot (Keener and Sizensky, 2005).

Latimer and Lovejoy (1989) have hypothesized that the lateral process may also serve to increase the surface area of the foot in order to aid in force dissipation during locomotion.

The peroneal, or fibular, tubercle is a bony projection located on the lateral surface of the calcaneal body. The peroneus longus and peroneus brevis tendons, which function in plantarflexion and eversion of the foot, are separated by the peroneal tubercle (Agarwal et al., 1984; Hofmeister et al., 1996; Hyer et al., 2005; Gill et al., 2014; Shibata et al., 2014). Previous research has suggested that the primary function of the peroneal tubercle is to act as a fulcrum for the peroneus longus tendon, directing it towards the cuboid, but the peroneal tubercle also functions as the insertion site for the inferior peroneal reticulum and separates the sheaths of the peroneus longus and brevis tendons (Berenter and Goldman, 1989; Hofmeister et al., 1996; Ruiz et al., 1993).

The calcaneus articulates with the talus to form the subtalar joint. The subtalar joint is fundamental to the transmission of rotations from the foot to the leg during locomotion. The articulation between the calcaneus and the talus can be seen in Figure 2.2 and the subtalar joint (in situ) can be seen in Figure 2.5. 


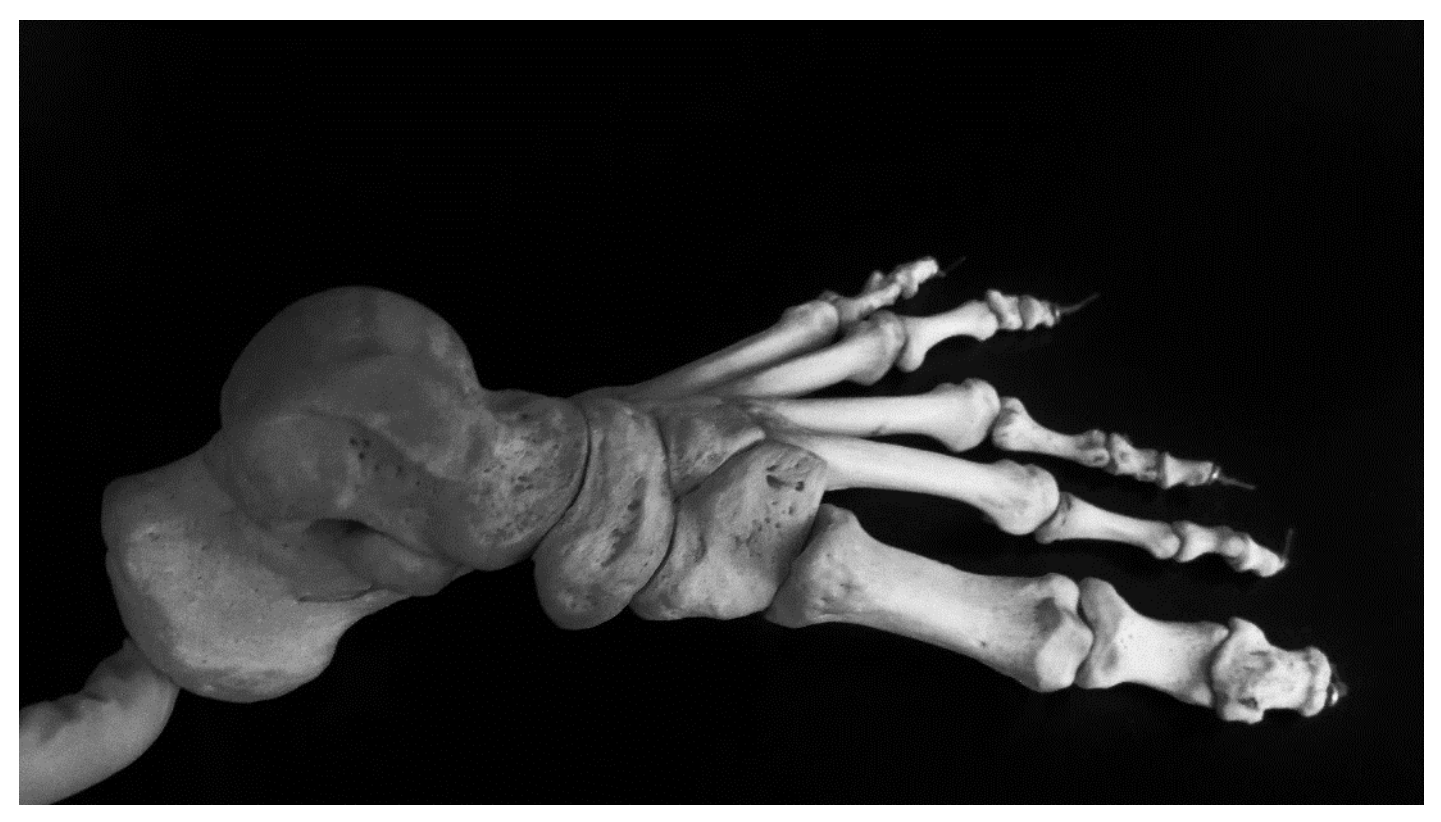

Figure 2.5 Medial view of the left human foot depicting the articulation between the calcaneus and the talus to form the subtalar joint. (Photo by Shelby Scott)

The calcaneus articulates with the talus on two separate articular surfaces: the sustentaculum tali (bearing a middle and anterior facet) and the posterior facet. The sustentaculum tali is located on the medial side of the calcaneus and articulates with the talus as it functions to support the talar head (Keener and Sizensky, 2005; Mahato, 2011; Sharadra et al., 2012; Viladot et al., 1984). The sustentaculum tali bears a middle facet and an anterior facet, which influence subtalar joint stability (Sharadra et al., 2012). Often, the anterior facet and the middle facet are joined but are separate from the posterior facet. In this scenario, the individual would display two facets (Piazza, 2005). However, there is substantial variation in the configuration of the anterior, middle, and posterior facets; they may appear as three separate facets (i.e. an anterior facet, a middle facet, and a posterior facet) or as one contiguous facet (Bunning and Barnett, 1965; Keener and Sizensky, 2005; Piazza, 2005; Saadeh et al., 2000; Viladot et al., 1984). The 
morphological variability of the anterior articular facets may be influenced by factors such as biological affinity and/or sex (Bunning and Barnett, 1965; Saadeh et al., 2000; Sharadra et al., 2012).

The sustentacular sulcus, or groove, is located inferior to the sustentaculum tali. The tendon of the flexor hallucis longus muscle is located within the sustentacular sulcus and is responsible for plantarflexion of the foot and of the hallux (Keener and Sizensky, 2005; Mahato, 2011).

\subsubsection{Talus: Anatomy and Function}

The talus is the second largest of the tarsal bones and is located inferior to the tibia and fibula and superior to the calcaneus. The talus articulates inferiorly with the calcaneus, distally with the navicular, and superiorly with the tibia and fibula. The talus is comprised of three major structures: the talar head, the talar neck, and the talar body (Figure 2.6). 


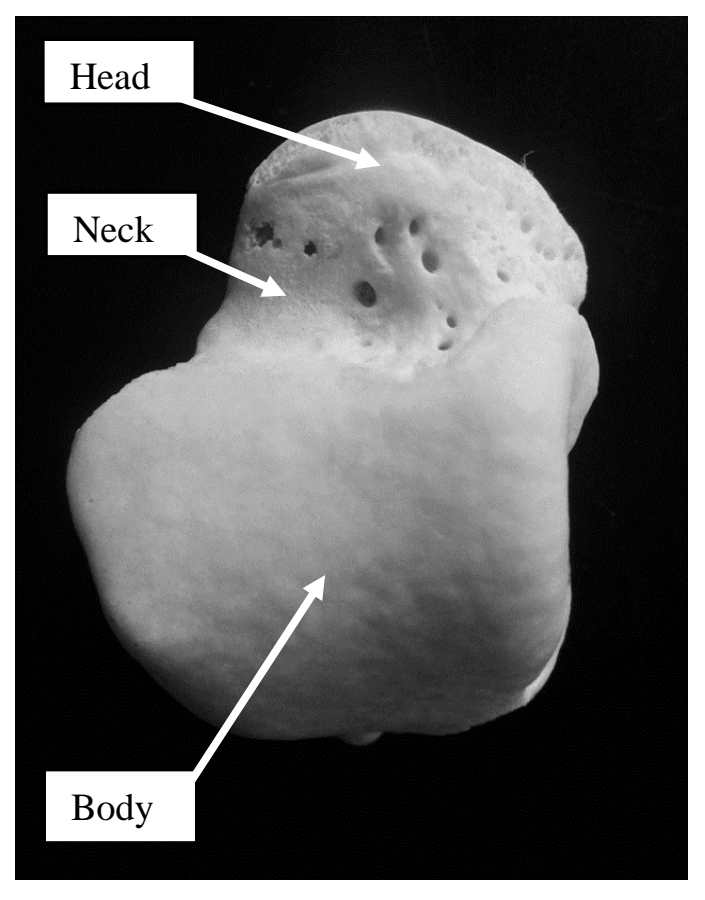

Figure 2.6 Dorsal (superior) view of a typical left talus. (Photo by Shelby Scott)

The talar neck connects the head of the talus with the body of the talus. This junction subtends an angle of approximately $120^{\circ}$ (measured between the axis of the talar head and a transverse coronal plane passing across the superior articular surface of the talus) (Mahato and Murthy, 2012). Previous studies have demonstrated that the angle of the talar neck may change throughout life and may be influenced by the amount of weight placed on the talus (de Palma et al., 2003; Mahato, 2011; Mahato and Murthy, 2012; Sarrafian, 1993). The talar neck may display articular facets (also known as "squatting facets") that are formed through contact with the anterior surface of the distal tibia as a result of strong dorsiflexion of the foot at the talocural joint (Barnett, 1954; Oygucu et al., 1998; Pandey and Singh, 1990; Rao Prasada, 1966; Satinoff, 1959; Singh, 1959;

Thomson, 1889, 1890; Trinkaus, 1975). The talocrural (ankle) joint is formed through the articulation of the talus, tibia, and the fibula, and assists in transferring the weight of the 
body to the articulated foot during locomotion (Figure 2.7) (Dul and Johnson, 1985;

Hicks, 1953; Inkster, 1927; Isman and Inman, 1969; Leardini et al., 2001; Manter, 1941;

Piazza, 2005; Sharada et al., 2012).

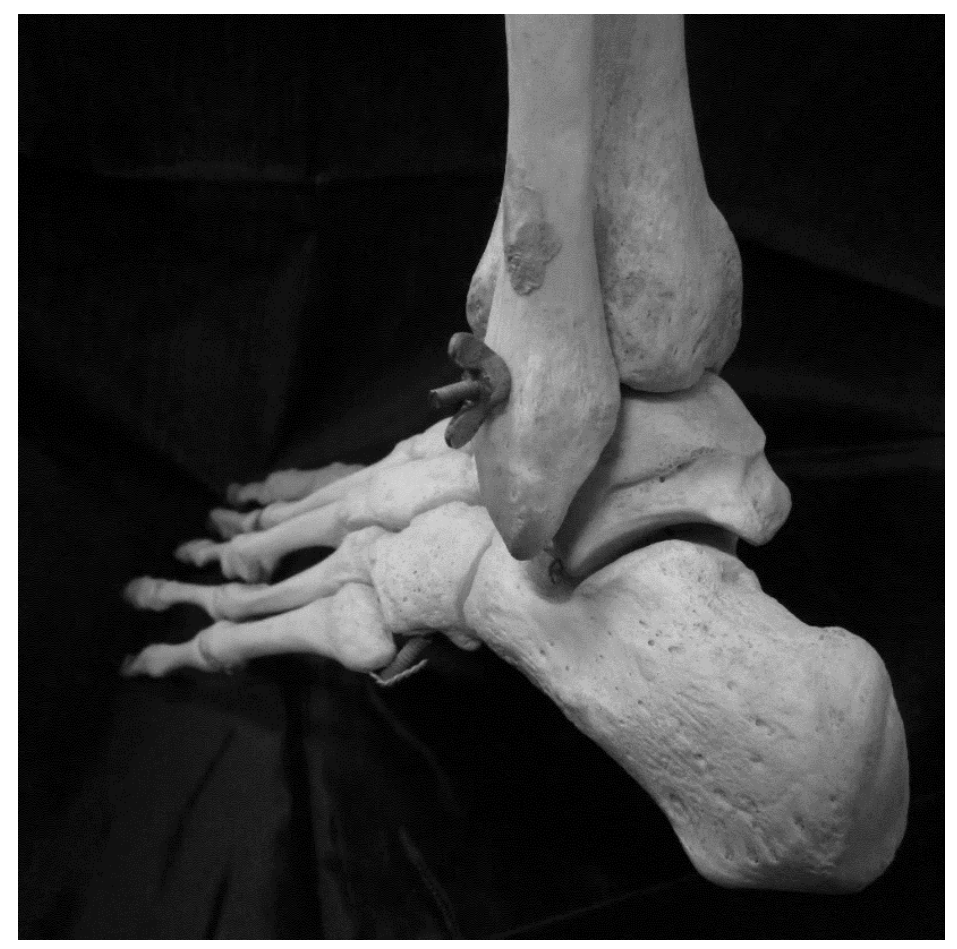

Figure 2.7 Posterolateral view of the left human foot depicting the articulation between the talus, the tibia, and the fibula to form the talocrural (ankle) joint. (Photo by Shelby Scott)

The talar body is square in shape and is located posterior to the talar neck. The trochlea, located on the talar body, is a wedge-shaped articular surface with two sides known as the lateral malleolar surface and the medial malleolar surface. The lateral malleolar surface articulates with the fibula and the medial malleolar surface articulates with the tibia forming the talocrural joint (Figure 2.7). The calcaneal or subtalar articular surfaces are located on the inferior aspect of the talar body and are often variable in shape and number (see section 2.1.2). Located on the posterior surface of the talar body is the 
groove for the flexor hallucis longus muscle. This groove transmits the tendon of the flexor hallucis longus muscle and is continuous with the sustentacular sulcus of the calcaneus (Keener and Sizensky, 2005; Mahato, 2011).

\subsection{Methods of Stature Estimation in Forensic Anthropology}

The estimation of stature is a critical factor in the identification of unknown skeletal remains, providing one aspect of an individual's biological profile. Forensic practitioners require precise and accurate methodologies for estimating stature in unidentified human remains. The theoretical foundation of stature estimation from skeletal remains dates back to 1755, when French anatomist Jean Joseph Sue (1710 to 1792) published data on maximum long bone length and stature in order to provide artists with information regarding body proportions (Stewart, 1979; Sue, 1755). These measurements were introduced to the medico-legal community by Matthieu Joseph Bonaventure Orfila (1787 to 1853). Orfila also added cadaveric and skeletal measurements to Sue's original data set, thus creating the 'Sue-Orfila stature estimate' database (Langley and Tersigni-Tarrant, 2017). In 1894, American anatomist Thomas Dwight distinguished between two methods of stature estimation, which are used in forensic anthropology today: anatomical and mathematical (Brandt, 2009; Dwight, 1984; Fully, 1956; Raxter et al., 2006; Trotter and Gleser, 1952; Wilson et al., 2010).

The anatomical method of stature estimation involves reassembling the skeleton in anatomical position, applying a correction factor for soft tissue, and measuring height (Langley and Tersigni-Tarrant, 2017). A major advantage of this method is that individual differences in body proportions are intrinsically incorporated. The anatomical approach to 
stature estimation is generally attributed to Fully (1956), who developed an anatomical reconstruction method by taking the following measurements from a French population (102 skeletonized males):

- Basion-bregma height of the cranium

- Maximum height of the corpus of the C2 to L5 vertebrae measured individually

- Anterior height of the first sacral segment

- Oblique (physiological) length of the femur

- Maximum length of the tibia without the tibial spine, and including the malleolus

- Articulated height of the calcaneus and the talus, from the most superior point on the talus to the most inferior point on the calcaneus

For the femur, the tibia, the calcaneus, and the talus, Fully's (1956) method cites that an average value from both left and right measurements should be used in calculating stature. Each of these individual measurements should then be summed to obtain skeletal height. In order to attain a final estimation of total stature (as opposed to total skeletal height), Fully (1956) presented soft tissue correction factors that then added to the calculated skeletal height value. The proposed soft tissue correction factors are as follows:

- Skeletal height equal to or less than $153.5 \mathrm{~cm}$, add $10 \mathrm{~cm}$

- Skeletal height between $153.6 \mathrm{~cm}$ and $165.4 \mathrm{~cm}$, add $10.5 \mathrm{~cm}$

- Skeletal height equal to or greater than $165.5 \mathrm{~cm}$, add $11.5 \mathrm{~cm}$

In 2006, Raxter et al. reviewed and revised the Fully (1956) anatomical reconstruction method. In a cadaveric study of Black American and White American individuals, Raxter 
et al. (2006) tested the accuracy and applicability of the method, and concluded that statures derived from the original Fully (1956) method underestimated total stature, in their sample, by approximately $2.4 \mathrm{~cm}$ on average. Earlier studies, which have reviewed the Fully technique, cite similar findings and have reported a systematic bias resulting in an underestimation of total stature (Bidmos, 2005; King, 2004). This suggests that the soft tissue correction factors, as outlined by Fully (1956), are too small. To remedy this, Raxter et al. (2006) clarified the measurement procedures of Fully (1956) and developed new formulae, for calculating total stature from skeletal height, which yielded stature estimates with a 95\% accuracy rate (within $4.5 \mathrm{~cm}$ ). Raxter et al. (2006) found no evidence of sex- or ancestry-related effects on the accuracy of stature estimation and therefore, sex and ancestry do not need to be known when utilizing these formulae. When the age of the individual is known, Raxter et al. (2006) recommend utilizing the following equation:

Total stature $(\mathrm{cm})=1.009 \times$ skeletal height $-0.0426 \times$ age +12.1 When the age of the individual is unknown, Raxter et al. (2006) recommend utilizing the following equation:

$$
\text { Total stature }(\mathrm{cm})=0.996 \times \text { skeletal height }+11.7
$$

While the anatomical method has been cited as providing accurate estimations of total stature, it requires a largely articulated skeleton, which is often not present in a forensic or disaster contexts (Gocha et al., 2013; Raxter et al., 2006; Ruff et al., 2012). As a result, relatively few studies continue to employ the anatomical approach, as regression formulae from individual skeletal elements (i.e. utilizing the mathematical method of stature estimation) are more applicable in a forensic or disaster context (Auerbach et al., 2009). 
The mathematical method of stature estimation involves the derivation of regression formulae, which demonstrate linear relationships between measurements of individual skeletal elements and stature. Some of the most commonly used regression equations are those developed by Trotter and Gleser (1952), based on Black Americans and White Americans from the Terry skeletal collection. Trotter and Gleser (1952) measured the left and the right upper and lower limb bones of Black American and White American males and females, and developed univariate and multivariate regression equations for stature estimation. Resulting estimates of total stature incorporate correction factors as well as an adjustment for the effect of aging on stature (Trotter and Gleser, 1952). Recently, Wilson et al. (2010) reviewed the Trotter and Gleser (1952) method and noted several problems with the application of these stature equations, the most problematic being the regional and temporal bias of their sample population (Wilson et al., 2010). The average birth date of individuals, specifically females, in the Terry skeletal collection is between 1850 and 1900, a period in which Meadows and Jantz (1999) cite Black Americans and White Americans as having the shortest stature. As a result, the equations derived from this population may not be accurate when applied to a modern American population. To remedy this, the Wilson et al. (2010) study used a contemporary American sample, and generated temporally-specific total stature estimation formulae using long bone lengths.

Stature estimation research in forensic anthropology typically utilizes the mathematical approach, which involves the extrapolation of living stature from individual skeletal elements through regression analyses (Auerbach et al., 2009). Using the regression formulae of:

$$
y(\text { stature })=b(x)+a
$$


where $b$ refers to the regression coefficient for the first variable (or measurement), $x$ refers to the first variable (or measurement), and $a$ refers to the constant, one can calculate stature from individual skeletal elements. In other words, the estimate of living stature is the sum of the products of the unstandardized coefficient and the magnitude of the corresponding measurement variables and the constant value, or intercept.

When regression formulae are calculated, the statistical output (model summary) provides a standard error of the estimate (SEE) value. To obtain a range for individual stature, rather than an absolute value, the standard error of the estimate can be added to, or subtracted from, the final estimate (y) (Bidmos and Asala, 2005; Bidmos, 2006). In forensic anthropology, a range for stature estimation is useful because it enhances the accuracy of an estimate by increasing the chance that the unidentified individual will fall within that particular range. The SEE can be defined as "a measure of expected accuracy of a stature estimate of an individual who belongs to the same population from which the equation was derived" (Trotter and Gleser, 1958: 115). Therefore, the SEE of a regression equation is a measure of the accuracy of the estimation of stature (i.e. how close the estimate is to the documented living stature). The lower the SEE as presented by a regression equation, the more accurate is the estimate of stature.

The statistical output (model summary) also provides Pearson correlation values ( $r$-values) when regression formulae are calculated. The r-value indicates the strength and direction of the relationship between measurement variables. For example, if a correlation coefficient of $0.99(r=0.99)$ was found when estimating stature from the calcaneus in a Black South African population, this would demonstrate that there is a strong relationship between measurements of the calcaneus and living stature, and would prove the accuracy 
of the calcaneus for stature estimation in a Black South African population (Bidmos and Asala, 2005).

One of the most important aspects of the mathematical approach is the notion of population-specificity regression formulae. Researchers are advised to be cautious when applying stature regression formulae to their study population if the methods were derived from a different population (Trotter and Gleser,1952; Wilson et al., 2010). Human proportions vary systematically between populations. Therefore, the most accurate mathematical estimates of stature will be obtained when the population being investigated is as similar as possible to the population that was used to derive the regression formulae (Holliday and Ruff, 1997; Wilson et al., 2010).

\subsubsection{Stature Estimation: Regression Formulae from the Long Bones}

Long bones (i.e. the humerus, ulna, radius, femur, tibia, and fibula) are most often used for estimating stature from unidentified skeletal remains as they provide the most accurate estimation of stature. The formulae for long bone estimation that are typically utilized by forensic anthropologists are those developed by Trotter and Gleser (1952) (see section 2.2.2) and Trotter (1970). The results of these studies have demonstrated a high correlation between the length of long bones and stature, i.e. correlation coefficients ranging from 0.63 to 0.84 in Black Americans and from 0.72 to 0.87 for White Americans; the highest single correlation is typically that of the femur (Steele, 1970; Steele and McKern, 1969; Trotter, 1970; Trotter and Gleser, 1952).

In disaster identification contexts, long bones are often found fragmented, which precludes any measurements of long bone length and, thus, the use of formulae for stature 
estimation from long bone length. To remedy this, researchers have used fragmentary long bones to derive regression equations for the estimation of stature in a number of populations (Müller, 1935; Simmons et al., 1990; Steele, 1970; Steele and McKern, 1969). Steele and McKern (1969) for example, examined the femur, tibia, and humerus in order to determine whether fragments of these skeletal elements provide an accurate estimation of stature in a prehistoric American Indian population. While the results of this research suggest fragments of the femur, tibia, and humerus show high accuracy rates for stature estimation, Steele and McKern (1969) note that these methods are problematic in practical forensic applications due to the difficulty in identifying the anatomical landmarks required for collecting the measurements. Simmons et al. (1990) revised the Steele and McKern (1969) method for stature estimation from fragmentary femora using a Black American and White American population. Simmons et al. (1990) used clearly defined standard measurements from the proximal, midshaft, and distal regions of the femur. The results of this analysis show correlation coefficients that range from 0.220 to 0.677. This suggests that fragmentary long bones have lower accuracy rates than when using intact long bones for stature estimation.

\subsubsection{Stature Estimation: Regression Formulae from the Skull}

Studies that have investigated the estimation of stature from measurements of the skull have shown varying degrees of accuracy across a number of populations (Chiba and Terazawa, 1998; Fully, 1956; González-Colmenares et al., 2016; Introna et al., 1993; Krishan, 2008; Patil and Mody, 2005; Torimitsu et al., 2016). Fully (1956) first used the skull for stature estimation in a French population as one of the skeletal elements measured when using the Fully method (see section 2.2.1). In 1993, Introna et al. 
measured the maximum anterior-posterior length and the maximum breadth of the skull in an Italian population (358 males) in order to evaluate the correlation between measurements of the skull and skeletal height. The results of this study indicate that the dimensions of the skull are positively correlated with skeletal height, with correlation coefficients ranging from 0.16 to 0.26 (Introna et al., 1993). In 1998, Chiba and Terazawa conducted a cadaveric study in a Japanese population (77 males, 47 females) in order to investigate the estimation of stature from the diameter (distance from the glabella to the external protuberance) and circumference (total length around the skull through the glabella and external protuberance) of the skull. The results of this study demonstrate correlation coefficients ranging from 0.32 to 0.53 (Chiba and Terazawa, 1998). In 2016, Torimitsu et al. also assessed the correlation between the skull and stature in a contemporary Japanese population (123 males, 105 females), using five cranial measurements: minimum frontal breadth, bi-zygomatic breadth, maximum cranial length, parietal chord, and maximum cranial breadth. The results of this study demonstrated higher correlation coefficients for stature estimation from parameters of the skull $(0.459$ to 0.764 ) than did the parameters of the skull measured by Chiba and Terazawa (1998).

Other studies have investigated cephalometric landmarks using lateral cephalogram radiography. Patil and Mody (2005) derived a regression equation for estimating stature from the maximum length of the skull (measured from the glabella to the opisthocranion) in a Central Indian population ( 75 males, 75 females). They found high accuracy rates for the estimation of stature using this equation, though absolute correlation coefficient values were not provided for this study. In a more recent study, utilizing lateral cephalogram radiography, González-Colmenares et al. (2016) investigated the accuracy of cephalo-facial measurements for stature estimation in a 
contemporary Colombian Mestizo population (54 males, 16 females). For males, there was a correlation from 0.28 to 0.47 between stature and the following measurements: nasion to menton, glabella to opisthocranion, basion to nasion, mastoidale to the sellanasion plane, and basion to anterior nasal spine. There were no significant correlations between the cephalometric measurements and stature in females (González-Colmenares et al., 2016). Overall, the literature suggests that measurements of the cephalic region (head) have a stronger correlation with stature than measurements of the facial region (González-Colmenares et al., 2016; Krishan, 2008; Krishan and Kumar, 2007;

Mahalakshmi and David, 2013; Patil and Mody, 2005).

\subsubsection{Stature Estimation: Regression Formulae from the Vertebrae}

Numerous studies have been published for the estimation of stature from the vertebrae in various populations (Dwight, 1894; Jason and Taylor, 1995; Nagesh and Kumar, 2006; Rodríguez et al., 2016; Terazawa et al., 1990; Tibbetts, 1981; Torimitsu et al., 2015). In 1894, Dwight published a study that introduced a method for estimating stature from the vertebral column in an unknown population. The results of this study indicated that the vertebral column can provide an accurate estimation of stature, though correlation coefficients were not produced (Dwight, 1894). The method published however, requires a fresh and articulated spine and, thus, this method is not applicable in a forensic anthropological context when human remains are skeletonized (Dwight, 1894).

In 1981, Tibbetts developed regression equations for the estimation of stature from the vertebral column and from segments of the vertebral column for a Black American population (100 males, 100 females). The vertebral column was divided into contiguous segments for analyses, containing from one to 23 vertebrae (second cervical 
vertebra [C2] to the fifth lumbar vertebra [L5]). The regression equations showed correlation coefficients that ranged from 0.18 to 0.64 , suggesting that the vertebral column may have high accuracy rates for the estimation of stature in the absence of more accurate predictors of stature, such as complete long bones.

Jason and Taylor (1995) developed regression equations for the estimation of stature from the vertebral column and vertebral segments of a Black American (43 males, 31 females) and White American (167 males, 58 females) population. The vertebral column was divided into the following vertebral segments: (i) cervical, (ii) thoracic, (iii) lumbar, (iv) thoraco-lumbar, and (v) cervico-thoraco-lumbar (Jason and Taylor, 1995). Regression equations were generated for both males and females of each population, showing correlation coefficients from 0.353 to 0.823 (Jason and Taylor, 1995). More recently, Nagesh and Kumar (2006) completed a study using a South Indian population (72 males, 45 females) that aimed to estimate stature using a methodology similar to that of Jason and Taylor (1995). The correlation coefficients ranged from 0.583 to 0.776 for males and from 0.325 to 0.708 for females. For both males and females, the total length of the vertebral column provided a more accurate estimate of stature than the individual vertebral segments (Nagesh and Kumar, 2006). This is consistent with the results of previous research and suggests that, when available, the vertebral column should be analyzed in its entirety for the most accurate estimation of stature (Dwight, 1894; Jason and Taylor, 1995; Tibbetts, 1981).

In a forensic context however, it is unlikely that the entire vertebral column will be present and, thus, it is necessary to develop methods of stature estimation using individual vertebrae or vertebral segments. The results of Nagesh and Kumar (2006) demonstrate that the cervical segment of the vertebral column has the lowest correlation 
coefficient (0.325 to 0.583$)$, and the lumbar segment of the vertebral column shows the highest correlation coefficient $(0.624$ to 0.659$)$. The low correlation coefficient value of the cervical segment is consistent with the results of Jason and Taylor (1995) in a Black American and White American population, and Torimitsu et al. (2015) in a Japanese population, who cited correlation coefficients from cervical vertebrae of 0.343 to 0.735 and 0.392 to 0.594 , respectively. The high correlation demonstrated between the lumbar segment and stature is in concurrence with Terazawa et al. (1990) and Tibbetts (1981). However, it contrasts with the results of Jason and Taylor (1995) who cited correlation coefficients from 0.537 to 0.686 for the lumbar vertebra. Overall, the literature suggests that, in the absence of an entire vertebral column, the lumbar segment of the vertebral column will provide the most accurate estimation of stature (Nagesh and Kumar, 2006; Terazawa et al. 1990, Torimitsu et al., 2015).

\subsubsection{Stature Estimation: Regression Formulae from Other Post Cranial Elements}

Researchers have used a variety of post cranial elements to estimate stature. Estimations of stature have been attempted using the sternum (Dwight, 1980; Marinho et al., 2012; Menezes et al., 2009, 2011; Singh et al., 2011; Yonguc et al., 2015; Zhang et al., 2015), clavicle (Balvir et al., 2012; Jit and Singh, 1956; Rajesh, 2014; Rani et al., 2011; Singh and Sohal, 1952; Torimitsu et al., 2017), scapula (Campobasso et al., 1998;

Giurazza et al., 2013; Shulin and Fangwu, 1983; Torimitsu et al., 2015; Zhang et al., 2016), metacarpals (Kimura, 1992; Meadows and Jantz, 1992; Musgrave and Harneja, 1978; Wilbur, 1998; Zaher et al., 2011), hand phalanges (Habib and Kamal, 2010; Jasuja and Singh, 2004; Jee and Yun, 2015; Shintaku and Furuya, 1990), sacrum (Fully, 1956; Giroux and Wescott, 2008; Karakas et al., 2011; Pelin et al., 2005; Pininski and Brits, 
2014; Torimitsu et al., 2014) and the metatarsals (Bidmos, 2008; Byers et al., 1989; Cordeiro et al., 2009; De Groote and Humphrey, 2011; Rodríguez et al., 2013). These studies demonstrate that postcranial elements, other than long bones, should be used with caution for the estimation of stature.

Dwight (1890), an American anatomist from Harvard University, was the first to investigate the relationship between the sternum and stature in White and Black American populations. Recent studies have also examined the accuracy of the sternum for the estimation of stature in various populations (Marinho et al., 2012; Menezes et al., 2009, 2011; Singh et al., 2011; Yonguc et al., 2015; Zhang et al., 2015). Menezes et al. (2009, 2011) derived linear regression equations for stature estimation from the sternum in 35 males and 40 females of a South Indian population. Menezes et al. $(2009,2011)$ concluded that sternum length was correlated with stature as they produced correlation coefficients of 0.638 in males and 0.639 in females. Other studies have demonstrated similar correlation coefficients in a Northwest Indian population (Singh et al., 2011) and in a Western Chinese population (Zhang et al., 2015). More recently, Yonguc et al. (2015) investigated the accuracy of the sternum for stature estimation in a Turkish population (65 males, 30 females). This study demonstrated correlation coefficients of up to 0.721 in Turkish males, and up to 0.740 in Turkish females. The results of these studies (Dwight, 1980; Menezes et al., 2009, 2011; Singh et al., 2011; Yonguc et al., 2015; Zhang et al., 2015) suggest that the sternum is an accurate predictor stature in the absence of more accurate skeletal elements. However, Marinho et al., (2012), who studied stature estimation from the sternum in a Portuguese population and found correlation coefficient values of only 0.329 , state that the sternum has limited forensic value and does not have high accuracy rates for stature estimation in this population. Therefore, further studies are 
needed to better understand the accuracy of the sternum for stature estimation in different populations.

The clavicle has also been investigated for its accuracy for stature estimation in various populations (Balvir et al., 2012; Jit and Singh, 1956; Rajesh, 2014; Rani et al., 2011; Singh and Sohal, 1952; Torimitsu et al.,2017). Singh and Sohal (1952) were the first researchers to investigate the accuracy of the clavicle for stature estimation, using maximum length of the clavicle to predict stature in a Punjabi population. In 1956, Jit and Singh re-examined the study of Singh and Sohal (1952), and found correlation coefficients ranging from 0.56 to 0.72 . The authors of this study concluded that the clavicle was not an accurate predictor of stature in a Punjabi population (Singh and Sohal, 1952). However, more recent studies have investigated the correlation between length of the clavicle and stature in various Indian populations and concluded that the clavicle may show high accuracy rates for stature estimation if more accurate skeletal elements (e.g. long bones) are not available (Balvir et al., 2012; Rajesh, 2014; Rani et al., 2011). Torimitsu et al. (2017) reached a similar conclusion when investigating the correlation between measurements of the clavicle and stature estimation. They used threedimensional computed tomographic images in a Japanese population (131 males, 118 females) and found correlation coefficients ranging from 0.510 to 0.816 . Overall, however, the literature suggests that the clavicle is not as accurate for stature estimation as other skeletal elements.

Early studies of stature estimation using measurements of the scapula can be attributed to Shulin and Fangwu (1983) and Campobasso et al. (1998). In 1983, Shulin and Fangwu took the morphological breadth of scapulae in a Southern Chinese population and concluded that the scapula is not as accurate for stature estimation as other skeletal 
elements, such as the os coxa. In 1998, Campobasso et al. investigated the correlation between measurements of the scapula (i.e. maximum length, maximum breadth, maximum acro-coracoid distance, maximum length of acromion, maximum length of coracoid, length of glenoid cavity, and width of glenoid cavity) and stature in a southern Italian population (40 males, 40 females). From this study, Campobasso et al. (1998) concluded that the scapula shows high accuracy rates for stature estimations with resulting correlation coefficients ranging from 0.23 to 0.62 . More recent studies support this conclusion, demonstrating correlation coefficients from 0.70 to 0.74 in an Italian population (longitudinal scapular length; Giurazza et al., 2013), 0.749 to 0.816 in a Japanese population (left longitudinal scapular length; Torimitsu et al., 2015), and 0.529 to 0.604 in a Chinese population (multiple regression) (Zhang et al., 2016). More studies are needed to determine the accuracy of scapular measurements for stature estimation in different populations.

Several studies have utilized the metacarpals for adult stature estimation with high rates of accuracy (Karaman et al., 2008; Meadows and Jantz, 1992; Musgrave and Harneja, 1978; Zaher et al., 2011). Musgrave and Harneja (1978) measured the interarticular length of each of the five metacarpal bones in a British population (120 males, 46 females) and derived regression equations for the estimation of stature. The results of this study demonstrate correlation coefficients ranging from 0.53 to 0.67 in males, and from 0.49 to 0.84 for females. Meadows and Jantz (1992) completed a similar study for a Black American (53 males, 55 females) and a White American (56 males, 48 females) population and also found the metacarpals to be an accurate predictor of stature, with correlation coefficient values ranging from 0.565 to 0.828 . Karaman et al. (2008) also revealed significant correlation coefficient values for the estimation of stature in a 
Turkish population (54 males, 46 females) using the second metacarpal length (0.785) and the third metacarpal length (0.743). However, a study by Zaher et al. (2011) revealed a slightly weaker correlation between measurements of the metacarpals and stature. Zaher et al. (2011) also found low correlation coefficient values between the length and the breadth of the second and the third metacarpal and stature in an Egyptian population (82 males, 75 females), ranging from 0.16 to 0.40 in Egyptian females and from 0.10 to 0.287 in Egyptian males. Overall, the literature suggests that metacarpal dimensions, particularly of the second and third metacarpals, are not highly accurate predictors of stature (Karaman et al., 2008; Meadows and Jantz, 1992; Musgrave and Harneja, 1978; Zaher et al., 2011).

The phalanges of the hand have also been investigated for stature estimation in a number of populations (Habib and Kamal, 2010; Jasuja and Singh, 2004; Jee and Yun, 2015; Shintaku and Furuya, 1990). Shintaku and Furuya (1990) investigated the correlation between the length of the proximal phalanges of the hand and stature in 231 Japanese women. The authors found a statistically significant correlation, with correlation coefficient values ranging from 0.521 to 0.696 (Shintaku and Furuya, 1990). Similarly, Jasuja and Singh (2004) found a statistically significant correlation between the phalanges (of each finger) and stature in a Punjabi Jat Sikh population (30 males, 30 females), with correlation coefficient values from 0.327 to 0.502 in males and from 0.336 to 0.529 in females. However, when measurements of phalange length were taken from an inked handprint, rather than directly from the skeletal element, correlation coefficient values increased to 0.394 to 0.652 in males and to 0.376 to 0.577 in females (Jasuja and Singh, 2004). Overall, the literature suggests that total hand length is more accurate for stature 
estimation than individual lengths of the hand phalanges (Habib and Kamal, 2010; Jee and Yun, 2015).

Fully (1956) was the first researcher to use the sacrum for stature estimation; he measured it as one of the skeletal elements employed as a part of the Fully method (see section 2.2.1). Pelin et al. (2005) were the first to publish information on the relationship between stature and sacral and coccygeal vertebrae dimensions through lateral magnetic resonance images taken from 42 Turkish males. The results of this study show correlation coefficient values from 0.088 to 0.677 , with overall sacral height and the sum of vertebral dimensions providing the most accurate estimations of stature as compared to individual vertebral measurements (Pelin et al., 2005). Giroux and Westcott (2008) completed a similar study in 2008 , measuring anterior length of the sacrum from dry bone in a Black American (57 males, 38 females) and White American (92 males, 60 females) population. The results of this study show correlation coefficient values from 0.13 to 0.46 . More recent studies that investigate the relationship between stature and dimensions of the sacrum show correlation coefficients ranging from 0.17 to 0.54 in an Anatolian Caucasian population (Karakas et al., 2011), from 0.16 to 0.66 in a Black South African and White South African population (Pininski and Brits, 2014), and from 0.587 to 0.716 in a Japanese population (Torimitsu et al., 2014). These studies demonstrate results that are consistent with the previous literature (Giroux and Westcott, 2008; Pelin et al., 2005). Overall, the literature suggests that the sacrum should only be used for stature estimation when more accurate skeletal elements are not available for analyses (Giroux and Wescott, 2008; Karakas et al., 2011; Pelin et al., 2005; Pininski and Brits, 2014; Torimitsu et al., 2014). 
There has been some research in a number of populations examining the accuracy of metatarsals for stature estimation (Bidmos, 2008; Byers et al., 1989; Cordeiro et al., 2009; De Groote and Humphrey, 2011; Rodríguez et al., 2013). Byers et al. (1989) investigated the relationship between metatarsal length and stature on a sample of male and female Black Americans (13 males, 9 females) and White Americans (57 males, 51 females). They demonstrated significant correlation coefficients ranging from 0.58 to 0.89. In 2008, Bidmos investigated stature estimation using linear measurements of metatarsals in an indigenous South African (60 males, 53 females) and White South African (58 males, 55 females) population. The results from Bidmos (2008) support the conclusions of Byers et al. (1989) with correlation coefficient values ranging from 0.44 to 0.76. More recent studies demonstrate similar findings, suggesting that the metatarsals are accurate for stature estimation (Cordeiro et al., 2009; De Groote and Humphrey, 2011; Rodríguez et al., 2013).

\subsubsection{The Potential for Stature Estimation from the Calcaneus and the Talus}

In the human body, the calcaneus and the talus are the largest tarsal bones and have been shown to produce high accuracy rates for human identification as they are often well preserved and able to withstand high tensile forces (Bidmos, 2006; Bidmos and Asala, 2005; Hall and Shereff, 1993). This increased preservation is related to the increased strength and density of the bones trabeculae and the smaller surface area of the calcaneus and the talus. Small, compact bones, such as the calcaneus and the talus, are often recovered intact as the bones smaller surface area expose less bone to destructive taphonomic elements (Bidmos and Asala, 2005). The calcaneus and the talus are often 
encased in socks and/or shoes, which serve as protection for the feet (Peckmann et al., 2015a,b; Pickering, 1986; Saul and Saul, 2005). Furthermore, the unique morphology of the calcaneus and the talus allow for rapid and accurate anatomical identification during excavation (Lee et al., 2012; Susman, 1983). These characteristics suggest that the calcaneus and the talus may provide accurate results for the estimation of stature and identification of unknown human remains.

Despite this, the calcaneus and the talus are underrepresented in the forensic and anthropological literature for stature estimation. Yet, these bones have been shown to be accurate predictors of individual stature. Regression equations for the estimation of stature using measurements of the calcaneus and the talus have been generated for Black American, White American, Black South African, and White South African populations with a high degree of accuracy (Bidmos, 2006; Bidmos and Asala, 2005; Holland, 1995; Pablos et al., 2013).

In 1995, Holland examined both the calcanei and tali of 60 Black Americans (30 males, 30 females) and 60 White Americans (30 males, 30 females) to determine the accuracy of certain measurements of the calcaneus and of the talus for stature estimation. Regression formulae for the calcaneus and for the talus were created for Black Americans and White Americans separately. Holland (1995) utilized Black American individuals from the Hamann-Todd Collection at the Cleveland Museum of Natural History. These individuals are representative of a mixed temporal population, i.e. an historic Black American population and a modern early $20^{\text {th }}$ century Black American population. The Black American population shows birth dates ranging from 1908 to 1939 (personal communication, LM Jellema, Collections Manager, Cleveland Museum of Natural History). The results of this study demonstrate that maximum length of the calcaneus 
(MCAL), posterior length of the calcaneus (PCAL), and maximum length of the talus (MTAL) are positively correlated with stature, with correlation coefficients of 0.723 , 0.817, and 0.731, respectively. In 2013, Pablos and colleagues completed a similar study using a more robust statistical method known as least trimmed squares (LTS). These authors examined both the calcanei and tali of 48 Black Americans (23 males, 25 females) and 46 White Americans (25 males, 21 females) to determine if the maximum length of the calcaneus and the maximum length of the talus were correlated with stature. The results of this study show correlation coefficients less than those found by Holland (1995), with the maximum length of the calcaneus demonstrating correlation coefficients ranging from 0.205 to 0.738 and the maximum length of the talus demonstrating correlation coefficients ranging from 0.1472 to 0.663 . To date, these are the only studies employed for stature estimation using osteometric criteria of both the calcaneus and the talus.

However, studies have been completed using only measurements of the calcaneus for stature estimation. In 2005, Bidmos and Asala examined the calcanei of 116 Black South Africans (60 males, 56 females) in order to examine which measurements of the calcaneus could provide the most accurate estimation of individual stature. Nine parameters were measured for each calcaneus: maximum length (MAXL), load arm length (LAL), minimum breadth (MINB), body height (BH), maximum height (MAXH), middle breadth (MIDB), dorsal articular facet length (DAFL), dorsal articular facet breadth (DAFB), and cuboidal facet height $(\mathrm{CFH})$. The results of this study show correlation coefficients ranging from 0.27 to 0.47 for Black South African males, and from 0.35 to 0.65 for Black South African females for individual variables of the calcaneus. For Black South African males, the MIDB measurement demonstrated the 
highest correlation with individual stature (0.47), while the DAFL measurement demonstrated the highest correlation with individual stature (0.65) for Black South African females. When combinations of variables of the calcaneus were tested (i.e. multiple regression analyses), correlation coefficients ranged from 0.52 to 0.60 for Black South African males (with the highest correlation using the MAXL, MAXH, MIDB and DAFB measurements) and from 0.72 to 0.77 for Black South African females (with the highest correlation using the MAXL, LAL, BH, and DAFL measurements). Overall, Bidmos and Asala (2005) conclude that the MAXL and MIDB measurements are individually the most accurate measurements of the calcaneus for stature estimation and that the accuracy of stature estimation in a Black South African population is enhanced when multiple calcaneal variables are analyzed. In 2006, Bidmos completed a similar study on a White South African population (85 individuals; 41 males, 44 females). The results of this study are similar to those found by Bidmos and Asala (2005); correlation coefficients for individual calcaneal variables ranged from 0.45 to 0.72 for White South African males and from 0.50 to 0.75 for White South African females, with the MAXL measurement demonstrating the highest correlation with individual stature for both males (0.72) and females (0.75). When combinations of variables of the calcaneus were tested (i.e. multiple regression analyses), correlation coefficients ranged from 0.74 to 0.78 for White South African males (with the highest correlation using the MAXL, BH, CFH measurements) and from 0.76 to 0.81 for White South African females (with the highest correlation using the BH, DAFB, MAXL, and MINB measurements).

More recently, Zhang et al. (2016) studied the calcanei of 293 Han Chinese individuals (121 males, 172 females) in order to examine which measurements of the calcaneus could provide the most accurate estimation of individual stature and sex. Five 
parameters were measured for each right calcaneus, including: maximum length $\left(X_{1}\right)$, body length $\left(X_{2}\right)$, maximum height $\left(X_{3}\right)$, minimum height $\left(X_{4}\right)$, and cuboidal facet height $\left(X_{5}\right.$ ) These measurements were recorded from computed radiography $(\mathrm{CR})$ technology, rather than from dry bone as was the protocol of previous studies (Bidmos, 2006; Bidmos and Asala, 2005; Holland, 1995). The results of the Zhang et al. (2016) study show correlation coefficients ranging from 0.25 to 0.61 for Han Chinese males and from 0.31 to 0.62 for Han Chinese females for individual variables of the calcaneus. For both Han Chinese males and Han Chinese females, the maximum length $\left(X_{1}\right)$ measurement demonstrated the highest correlation with individual stature ( 0.61 for males, 0.62 for females). When combinations of variables of the calcaneus were tested (i.e. multiple regression analyses), correlation coefficients were 0.66 for Han Chinese males and Han Chinese females (using all measurement variables in each equation). Overall, Zhang et al. (2016) conclude that the calcaneus is not as accurate for stature estimation in a Han Chinese population as it was found to be in previous studies (i.e. Bidmos, 2006; Bidmos and Asala, 2005). This may be because the data utilized in the Zhang et al. (2016) study were obtained from computed radiography $(\mathrm{CR})$ technology; it has been shown that taking measurements from digital imaging may not be as accurate as taking the measurements directly from dry bone (Berg et al., 2007; Guenoun et al., 2012; Lagravère et al., 2010; Major et al., 1994; Spoor et al., 2000).

Overall, studies that have investigated the accuracy of the calcaneus and the talus for stature estimation (i.e. Bidmos, 2006; Bidmos and Asala, 2005; Holland, 1995; Pablos et al., 2013) indicate that these bones are accurate predictors of individual stature and may be used for stature estimation in the absence of intact long bones. However, these studies have shown that methods of stature estimation to be population specific. The current 
project is important as, currently, there are no calcanei or talar regression formulae for stature estimation that employ a Thai population. Therefore, research is needed in order to determine the accuracy of these bones for stature estimation in a Thai population.

\subsection{Climate Change in Thailand: Implications for Forensic Anthropology}

For decades, global climate change has been cited as a major cause for concern. Scientists have listed various greenhouse gases which alter the climate of the Earth through absorption of energy and re-emission, including carbon dioxide $\left(\mathrm{CO}_{2}\right)$, nitrous oxide $\left(\mathrm{N}_{2} \mathrm{O}\right)$, and methane $\left(\mathrm{CH}_{4}\right)$ (Bolin et al., 1986; Montzka et al., 2011; Rodhe, 1990; Solomon et al., 2010). The impact of global climate change includes overall increases in global temperature and rainfall, rising sea levels, and the erosion of natural protective barriers of coastlines such as mangroves and coral reefs, all of which may contribute to an increase in severe climate change events and natural disasters (Anthes et al., 2006; Marks, 2011; Van Aalst, 2006; Webster et al., 2005).

The impacts of climate change are particularly severe in developing countries and coastal regions. The Intergovernmental Panel on Climate Change (IPCC) has listed Southeast Asia as one of the most vulnerable regions in the world and states that Thailand is particularly susceptible to the effects of climate change, namely coastal flooding and natural disasters such as tsunamis and cyclones, because of the natural low water level and small tidal range of the region. An analysis of meteorological data collected from the Thai Meteorological Department between 1980 and 2009 indicates an upward trend in monthly average temperatures and annual rainfall amount in Thailand (Thavorntam and Tantemsapya, 2013). The IPCC noted that this upward drift is likely due to an increase in 
greenhouse gas concentrations caused by anthropogenic activity (Intergovernmental Panel on Climate Change, 2007).

A number of researchers have demonstrated a link between global climate change and an increase in the frequency and severity of natural disasters such as hurricanes, tsunamis, and tropical cyclones (Anthes et al., 2006; Van Aalst, 2006; Webster et al., 2005). One of the most infamous natural disasters that may be attributed to climate change effects in Thailand is the 'Boxing Day Tsunami' that occurred on December 26, 2004, killing 5,395 individuals in Thailand (James, 2005; Sribanditmongkol et al., 2017; Wright et al., 2015). Major flooding events have also been a cause for concern in Thailand, with the city of Chiang Mai in Northern Thailand flooding five times between August and November 2005 (Jarungrattanapong and Manasboonphempool, 2011; Jothityangkoon et al., 2013). Listed as the most severe floods in the region in over four decades, researchers have demonstrated that these floods were a direct result of climate change, as the high volume and long duration of rainfall caused riverbeds to overflow (Jarungrattanapong and Manasboonphempool, 2011).

Recent studies have also shown that the effects of climate change will continue to impact Thailand; flooding and natural disasters will multiply in both frequency and severity and will have negative impacts in terms of death and destruction (Arunrat and Pumijumnong, 2015; Jinsart et al., 2014; Jothityangkoon et al., 2013; Thavorntam and Tantemsapya, 2013; Wang et al., 2014). Recently, Wang et al. (2014) used a regional climate model called PRECIS (Providing Regional Climate for Impact Studies) for Thailand in order to identify changes in annual temperature and rainfall amount as a result of climate change. Results indicated that average annual temperature and rainfall amount will increase in the upcoming decades (Wang et al., 2014). Jinsart and Thepanondh 
(2014) also investigated future climate change scenarios using a computer based modelling system to simulate the impact of future climate change on temperature and rainfall amount. The results of this study also showed an overall increase in both annual temperature and rainfall amount in the next 50 to 100 years (Jinsart and Thepanondh, 2014). Overall, there is a consensus throughout the literature that climate change and climate change events will have a significant impact on the country of Thailand in the future (Arunrat and Pumijumnong, 2015; Jinsart et al., 2014; Jothityangkoon et al., 2013; Marks, 2011; Thavorntam and Tantemsapya, 2013; Wang et al., 2014).

Given that climate change events such as flooding and natural disasters are predicted to increase in both frequency and severity in Thailand, resulting in a higher number of deaths, it is crucial that population-specific methods of victim identification be

derived for a Thai population. Therefore, this project is timely and important as it will add to the methodologies employed by forensic anthropologists to assist with the identification of Thai victims in mass disasters.

\subsection{The Admissibility of Forensic Anthropology Methods in Court}

Forensic anthropologists are increasingly called upon to provide expert witness testimony for cases involving unknown human skeletal remains, and as a result, must consider the applications of their findings to legal matters. In the United States and in Canada, legislation dictates that in order to be considered admissible in a court of law, scientific techniques used by expert witnesses must adhere to the legal admissibility criteria outlined in Daubert and Mohan, respectively (Christensen, 2004; Holobinko, 
2012; Williams and Rogers, 2006). By adhering to these recognized standards, forensic anthropologists will ensure that their analyses are of the highest quality and reliability. Reliability refers to the degree of reproducibility of a method, or the ability of a method to produce consistent results. Reliability can be judged based on the use of tested scientific methods, description in peer-reviewed publications, and general scientific acceptance (Dirkmaat et al., 2008). Accuracy rates and levels of precision may determine the reliability of a given method (Christensen and Crowder, 2009). Accuracy refers to the measure of correct assessments that a method can estimate, and is typically reported in percentages (Sokal and Rohlf, 1995; Williams and Rogers, 2006). Precision refers to the ability of a method to produce consistent results regardless of whether the method is correct, and can be evaluated by measuring the degree of intra- and inter-observer error (Christensen and Crowder, 2009; Williams and Rogers, 2006). Osteological methods are considered to be reliable if they meet the established critical values of accuracy $(\geq 80 \%)$ and precision $(\leq 10 \%)$ (Williams and Rogers, 2006). For quality assurance purposes, accuracy and precision rates must be presented for all forensic anthropological research. This will establish transparency of newly developed methods and techniques and ensure that they are derived using scientifically accepted principles (Christensen and Crowder, 2009; Williams and Rogers, 2006). To ensure reliable, accurate, and precise methodological standards when developing new methods (e.g. those used for stature estimation), forensic anthropological research and testimony must now meet the standards of Daubert (in the United States) and Mohan (in Canada).

The first important ruling regarding the standard by which admissibility of scientific evidence was judged in the United States Federal Courts, was issued in the federal appeals court case Frye v. United States (293 F.2s 1013, 1923). The ruling of this 
case would eventually lead to the formation of the Daubert guidelines that are now standard in the United States. During the Frye v. United States (293 F.2s 1013, 1923) case, the Court, on the standard for admissibility of scientific expert witness testimony, ruled that: “...in admitting expert testimony deduced from a well-recognized scientific principle or discovery, the thing from which the deduction is made must be sufficiently established to have gained general acceptance in the particular field in which it belongs" (Frye v. United States, 54 App.D.C. 46, 293 F. 1013 1923). This ruling, also known as the Frye Rule, became the legal standard for determining admissibility of scientific evidence, and required that scientific methods and techniques be generally accepted within the field from which they were derived in order to be admissible (Christensen, 2004; Holobinko, 2012).

The primary shortcoming of the Frye standard was that newly developed and implemented techniques, such as DNA evidence, were not considered admissible (Christensen, 2004; Holobinko, 2012; Masten and Strzelczyk, 2001). In the 1993 court case Daubert v. Merrell Dow Pharmaceuticals, parents of Jason Daubert and Eric Schuller filed suit against the Merrell Dow pharmaceutical company on the premise that their child's birth defects were a result of the mothers' prenatal ingestion of the respondent's drug. Epidemiologic testimony, which demonstrated a causal relationship between prenatal use of the drug and birth defects, was deemed inadmissible according to the Frye Rule because it was based on a methodology that had not been generally accepted or reviewed by the scientific community. Daubert was dismissed based on the petitioner's failure to produce sufficient evidence (i.e. The Frye precedent), and the case was eventually appealed (Daubert v. Merrell Dow Pharmaceuticals, Inc., 509 U.S. 579, 1993). Ultimately, the United States Supreme Court concluded that the Frye Rule should 
not be considered a standard and that a reasonable minority opinion may be admitted into evidence, usually in the form of emerging research based on reliable and well-designed scientific studies (Christensen, 2004). A set of standards, known as the Daubert guidelines, were introduced in order to clarify some of the ambiguous tenants of the Frye Rule.

The Daubert guidelines for determining whether evidence is scientific and admissible are: (1) The content of testimony can be/has been empirically tested using the scientific method, (2) The technique or methodology has been subject to peer review, preferably in the form of publication in peer reviewed literature, (3) There are consistently and reliably applied professional standards and known or potential error rates for the technique, and (4) The Court may consider general acceptance by identifying the relevant scientific community and assessing the degree of acceptance (Christensen, 2004; Daubert v. Merrell Dow Pharmaceuticals, Inc., 509 U.S. 579, 1993). However, scientific evidence meeting the reliability and relevance criteria set out by the Daubert guidelines are not guaranteed admissibility; the Daubert criteria also prohibits the admission of scientific evidence where its “...potentially prejudicial, confusing, or misleading nature outweighs its probative value" (Holobinko, 2012: 394.e3).

Similar to the Daubert criteria, the Mohan admissibility criteria, created as a result of the 1994 Canadian Supreme Court trial Regina v. Mohan (Regina v. Mohan, 2 S.C.R. 9 File No. 23063), greatly impacted the admissibility of forensic evidence and scientific testimony in Canada. These criteria arose from a case in which the respondent, a paediatrician (Dr. Mohan), was charged with four counts of sexual assault from four female patients. Council of the respondent indicated intent to call a psychiatrist to testify that the perpetrator of the alleged offences is part of a limited group of individuals 
exhibiting sexually deviant tendencies and that the respondent did not possess the characteristics of such an individual. Ultimately, the evidence was ruled inadmissible and the respondent was found guilty. However, an appeal was filed, and the Ontario Court of Appeal stated that opinion evidence regarding likelihood of individual membership in a group characterized by abnormal proclivities was relevant and, therefore, should be judged based on relevance and not sufficiency (Holobinko, 2012; Regina v. Mohan, 2 S.C.R. 9 File No. 23063).

From the Mohan case, four governing factors of evidence admissibility, known as the Mohan criteria, were devised in order to allow Canadian trial judges to decide on the admissibility of expert testimony (Holobinko, 2012). The Mohan criteria are: (1) Relevance: the admission of expert evidence must be relevant to the case at hand; logically relevant evidence may be excluded if its probative value is overborne by its prejudicial effect, if the time required is not commensurate with its value, or if it can influence the trier of fact our of proportion to it's reliability, (2) Necessity: expert evidence, in order to be necessary, must be outside the experience/knowledge of a judge or jury, (3) The absence of any exclusionary rule: evidence must be given by a witness who has acquired special knowledge through study or experience in the matter; evidence must not be obtained illegally or inappropriately, and (4) A properly qualified expert: the expert witness must be qualified with proper training/certification in relevant scientific principles (Regina v. Mohan, 2 S.C.R. 9 File No. 23063).

As a result of the Daubert v. Merrell Dow Pharmaceuticals, Inc., and the Regina v. Mohan course cases, forensic anthropology in the United States and Canada has adopted a proactive approach towards testing and refining methodologies, evaluating intra- and inter-observer error, estimating error rates, and providing detailed statistical 
analyses. As a result, the methodologies used by forensic anthropologists have become more widely accepted by the legal system in terms of evidence admissibility and courtroom testimony (Lesciotto, 2015). 


\section{CHAPTER 3: MATERIALS AND METHODS}

\section{1 $\quad \underline{\text { Research Objectives }}$}

The current research study focuses on nine measurements of the calcaneus (Maximum Length, Maximum Height, Cuboidal Facet Height, Body Height, Minimum Breadth, Load Arm Length, Middle Breadth, Dorsal Articular Facet Breadth, and Dorsal Articular Facet Length) and one measurement of the talus (Maximum Length of the Talus) to establish an accurate method of living stature estimation for a contemporary Thai population. The objectives of this research are to:

1. investigate whether regression equations for the calcaneus, which were developed on a Black South African population (Bidmos and Asala, 2005), and regression equations for the talus, which were developed on a Black American population (Holland, 1995), can accurately predict the living stature of individuals from a contemporary Thai population.

2. develop population-specific simple regression and multiple regression equations for the calcaneus and the talus of the contemporary Thai population should the Black South African (Bidmos and Asala, 2005) and Black American (Holland, 1995) equations not accurately predict the living stature of the Thai individuals.

3. compare the results from the contemporary Thai population to other populations

\subsection{Materials and Method}

\subsubsection{Demographics of Skeletal Materials Utilized for this Research}

Measurements were taken from the paired calcaneus and talus of 233 adult skeletonized individuals (116 males, 117 females) of Thai ancestry from the Chiang Mai 
skeletal collection housed in the Forensic Osteology Research Center, at the Faculty of Medicine, Chiang Mai University, Thailand. All individuals utilized for this research must have had both the calcaneus and the talus available for inclusion in this study; if measurements could not be taken from the calcaneus, then the talus of that individual was excluded as well. Similarly, if measurements could not be taken from the talus of an individual then the calcaneus of that individual would be excluded as well.

In total, 466 individual bones $\left(\mathrm{N}_{\text {total bones }}=466 ; \mathrm{N}_{\text {calcaneus }}=233, \mathrm{~N}_{\text {talus }}=233\right)$ were examined from a total of 233 adult skeletonized individuals $\left(\mathrm{N}_{\text {individuals }}=233\right)$ (Table 3.1).

Table 3.1 Sample size distribution for the Thai sample utilized in this research study*.

\begin{tabular}{lllr}
\hline & Male & Female & Total Bones Examined \\
\hline $\mathrm{N}_{\text {calcaneus }}$ & 116 & 117 & 233 \\
$\mathrm{~N}_{\text {talus }}$ & 116 & 117 & 233 \\
$\mathrm{~N}_{\text {total bones }}$ & & 466 \\
\hline $\mathrm{N}_{\text {individuals }}$ & 116 & 117 & \\
* One paired calcaneus and talus was examined from each individual &
\end{tabular}

Individuals included in this research study were randomly selected with priority given to approximate equal numbers between the sexes (116 male calcanei and 116 male tali, 117 female calcanei and 117 female tali). Demographic information, including biological sex, living stature (height), age-at-death, year-of-death, and ancestry is available for each individual in the Chiang Mai skeletal collection. Total living stature was collected in the Department of Anatomy at Chiang Mai University at the time of body donation. Prior to soft tissue removal, a metal measuring tape was used to measure the length of the body, to the nearest centimeter, from the vertex of the head to the heel of the foot with the cadaver 
lying in a supine position (Mahakkanukrauh et al., 2011). The year-of-death for each individual in the collection is recorded in Buddhist years. To convert the year-of-death from Buddhist years to Christian years, the following formula must be used (as provided by the curators of the Chiang Mai skeletal collection):

Christian year-of-death $=$ Buddhist year-of-death -543

The year-of-birth is not recorded on the demographic data sheets. Instead, it was calculated by the current researcher by subtracting the age-at-death, as documented on the demographic data sheets, from the calculated Christian year-of-death. Individuals utilized for this research sample have birth dates between 1914 and 1995 and death dates between 2006 and 2014. Therefore, this skeletal sample is considered contemporary because all individuals lived during the $20^{\text {th }}$ and $21^{\text {st }}$ centuries.

Sub-adult individuals, or individuals under the age of 19 years of age (<19 years), were not included in this study. The talus reaches skeletal maturity with fusion of the talar epiphysis. This occurs at approximately 9 years of age in females and 12 years of age in males. The calcaneus reaches skeletal maturity with complete fusion at the calcaneal epiphysis between the ages of 15 years and 16 years in females and between the ages of 18 years and 20 years in males (Scheuer and Black, 2004). All individuals utilized for this research study were skeletally adult and between 19 years and 96 years of age (Figure 3.1). 


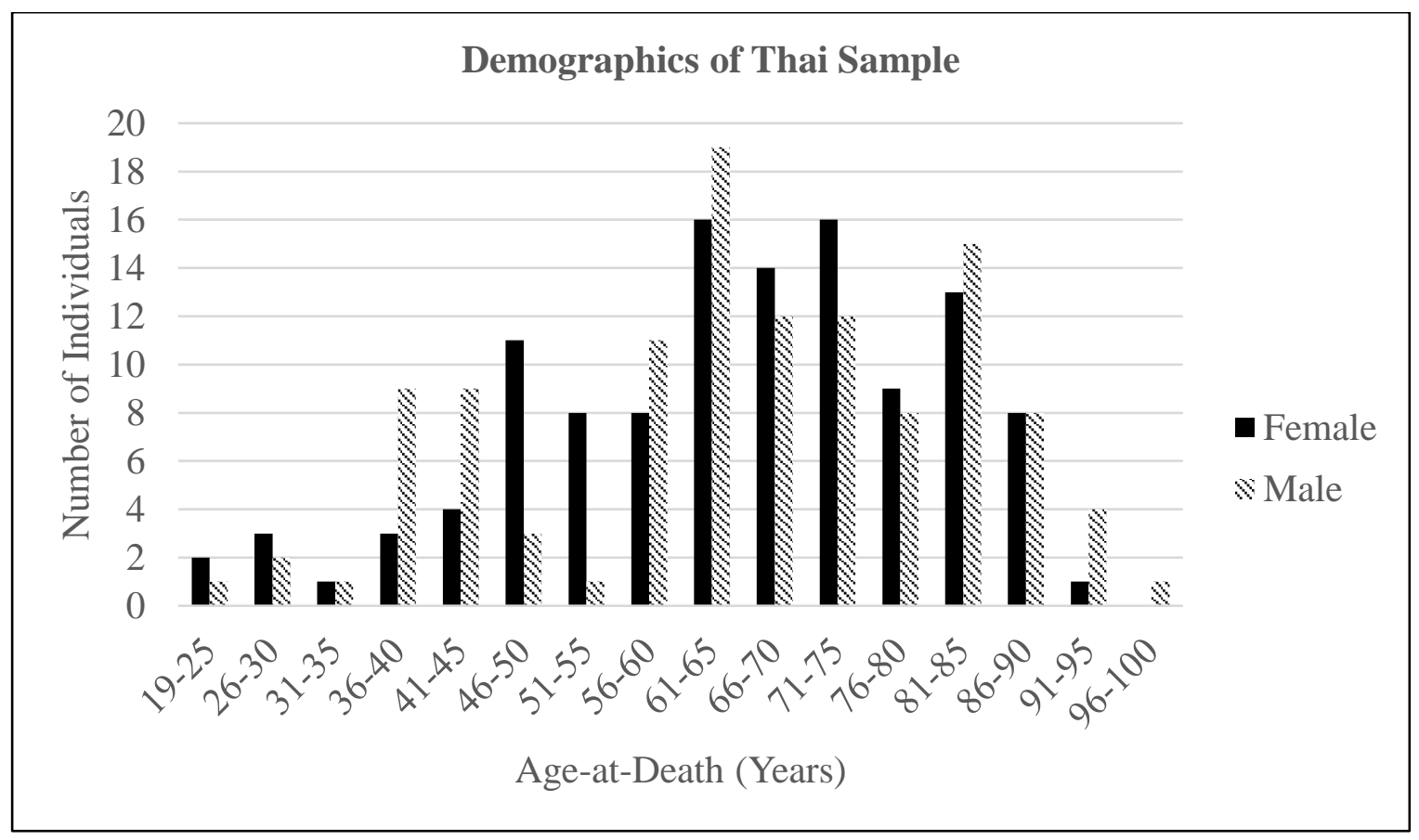

Figure 3.1 Distribution of age-at-death in the Thai skeletal sample utilized for this research study.

\subsubsection{Skeletal Materials Excluded from this Research}

Individuals were excluded from this study if both the calcaneus and the talus (from an individual pair) were not present for analysis, if the bones exhibited any form of taphonomic damage, post mortem trauma, or extreme pathologic remodeling of the morphometric characteristics which would inhibit the collection of accurate measurements (see Appendix B for examples of calcanei and tali which were excluded). Extreme pathologies included severe osteoarthritis, Diffuse Idiopathic Skeletal Hyperostosis (DISH), severe ankylosing spondylitis (AS) arthritis, and cancer (metastasis condition). Individuals over the age of 50 years (and possibly some younger individuals) may exhibit some form of osteoarthritic pathology as a result of natural mechanical stresses to the skeleton caused by physical activity, muscular strain, and age (Soren, 1993). The vast majority of skeletons in the Chiang Mai skeletal collection exhibited 
some osteoarthritic bone remodeling. Therefore, excluding these individuals would bias the independent sample from accurately representing the Thai population as a whole. Calcanei and tali exhibiting mild osteoarthritis, in which measured morphometric traits of the calcaneus and talus were not affected, were included in this study.

\subsubsection{Skeletal Measurements}

Following the protocol of Bidmos and Asala (2005), nine measurements ("measurement variables") were collected from the calcaneus: Maximum Length (MAXL), Maximum Height (MAXH), Cuboidal Facet Height (CFH), Body Height (BH), Minimum Breadth (MINB), Load Arm Length (LAL), Middle Breadth (MIDB), Dorsal Articular Facet Breadth (DAFB), and Dorsal Articular Facet Length (DAFL). Six of the nine measurement variables (Maximum Length, Cuboidal Facet Height, Load Arm Length, Middle Breadth, Dorsal Articular Facet Breadth, and Dorsal Articular Facet Length) follow the definitions by Martin and Knußman (1988). Three of the nine measurement variables (Maximum Height, Body Height, and Minimum Breadth) follow the definitions of Bidmos and Asala (2005). For detailed explanations of the calcaneal measurement variables utilized for this study, refer to Table 3.2. For visual depictions of the calcaneal measurement variables taken for this study refer to Figure 3.2 and Figure 3.3 . 
Table 3.2 Measurement variable descriptions of the calcaneus

\begin{tabular}{|c|c|}
\hline Measurement variable & Description \\
\hline $\begin{array}{l}\text { Maximum Length } \\
(\mathrm{MAXL})\end{array}$ & $\begin{array}{l}\text { The maximum distance between the most anterior } \\
\text { point of the calcaneus and the most posterior point of } \\
\text { the calcaneal tuberosity } *\end{array}$ \\
\hline $\begin{array}{l}\text { Maximum Height } \\
(\mathrm{MAXH})\end{array}$ & $\begin{array}{l}\text { The maximum distance between the most superior } \\
\text { point of the calcaneal tuberosity and the most inferior } \\
\text { point and of the calcaneal tuberosity } * *\end{array}$ \\
\hline $\begin{array}{l}\text { Cuboidal Facet Height } \\
(\mathrm{CFH})\end{array}$ & $\begin{array}{l}\text { The maximum distance between the most superior } \\
\text { point of the cuboidal articular facet and the most } \\
\text { inferior point of the cuboidal articular facet } *\end{array}$ \\
\hline $\begin{array}{l}\text { Body Height } \\
(\mathrm{BH})\end{array}$ & $\begin{array}{l}\text { The maximum distance between the superior surface } \\
\text { of the calcaneal body, and the inferior surface of the } \\
\text { calcaneal body } * *\end{array}$ \\
\hline $\begin{array}{l}\text { Minimum Breadth } \\
(\mathrm{MINB})\end{array}$ & $\begin{array}{l}\text { The minimum distance between the medial and } \\
\text { lateral surfaces of the superior part of the calcaneal } \\
\text { body } * *\end{array}$ \\
\hline $\begin{array}{l}\text { Load Arm Length } \\
\text { (LAL) }\end{array}$ & $\begin{array}{l}\text { The maximum distance between the most anterior } \\
\text { point of the calcaneus and the most posterior point of } \\
\text { the posterior articular facet } *\end{array}$ \\
\hline $\begin{array}{l}\text { Middle Breadth } \\
\text { (MIDB) }\end{array}$ & $\begin{array}{l}\text { The maximum distance between the most lateral } \\
\text { point of the posterior articular facet and the most } \\
\text { medial point of the sustentaculum tali } *\end{array}$ \\
\hline $\begin{array}{l}\text { Dorsal Articular Facet Breadth } \\
\text { (DAFB) }\end{array}$ & $\begin{array}{l}\text { The maximum distance from the most medial point } \\
\text { of the posterior articular facet to the most lateral } \\
\text { points of the posterior articular facet } *\end{array}$ \\
\hline $\begin{array}{l}\text { Dorsal Articular Facet Length } \\
\text { (DAFL) }\end{array}$ & $\begin{array}{l}\text { The maximum distance between the most posterior } \\
\text { point of the posterior articular facet and the most } \\
\text { anterior point of the posterior articular facet } *\end{array}$ \\
\hline
\end{tabular}

* Modified from Martin and Knußman, 1988

** Modified from Bidmos and Asala, 2005 


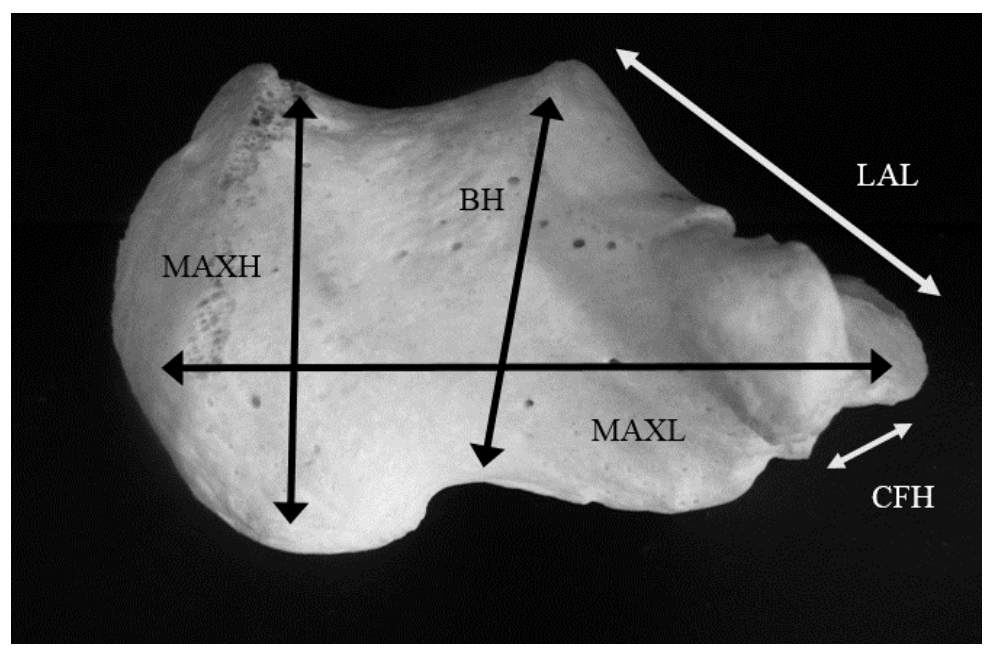

Figure 3.2 Medial view of a typical left calcaneus depicting calcaneal measurement variables: Maximum Length (MAXL), Maximum Height (MAXH), Cuboidal Facet Height (CFH), Body Height (BH), and Load Arm Length (LAL). (Photo by Shelby Scott)

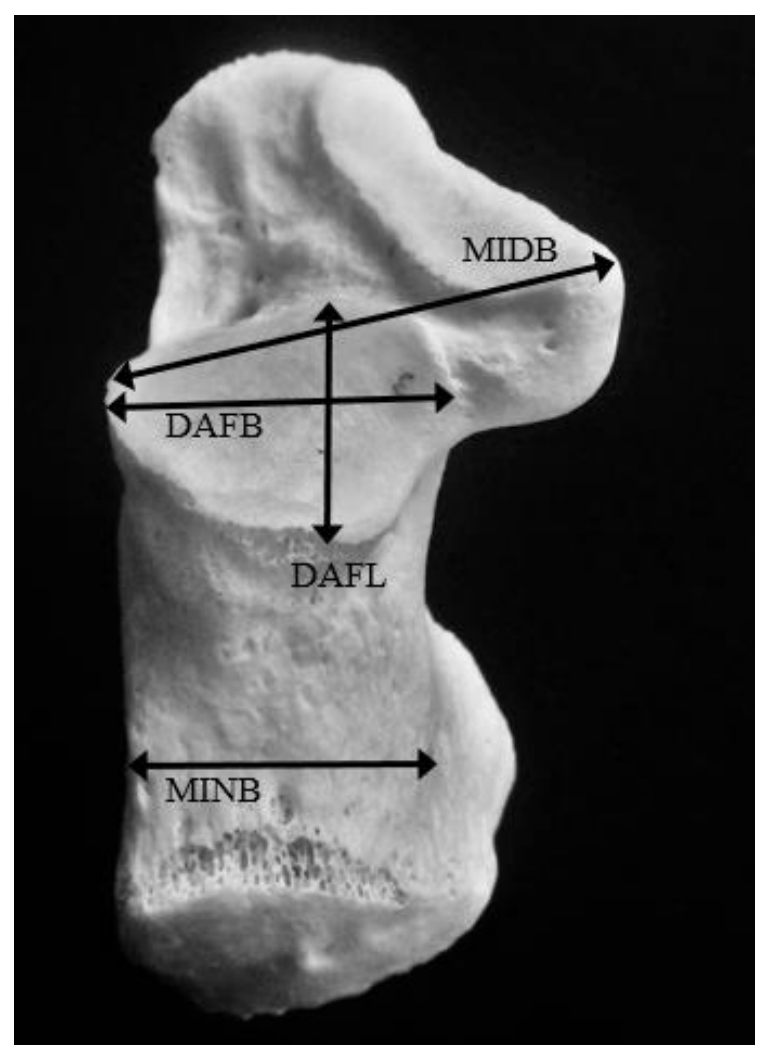

Figure 3.3 Superior view of a typical left calcaneus depicting calcaneal measurement variables: Minimum Breadth (MINB), Middle Breadth (MIDB), Dorsal Articular Facet Breadth (DAFB), and Dorsal Articular Facet Length (DAFL). (Photo by Shelby Scott) 
Following the protocol of Holland (1995), one measurement variable was collected for the talus: Maximum Length of the Talus (MTAL). For a detailed explanation of this talar measurement variable refer to Table 3.3. For a visual depiction of this talar measurement refer to Figure 3.4.

Table 3.3 Measurement variable description of the talus.

\begin{tabular}{|l|l|}
\hline Measurement variable & Description * \\
\hline Maximum Length of the Talus & $\begin{array}{l}\text { Maximum length between the most anterior point of } \\
\text { the head and the posterior tubercle }\end{array}$ \\
\hline
\end{tabular}

* Modified from Holland, 1995

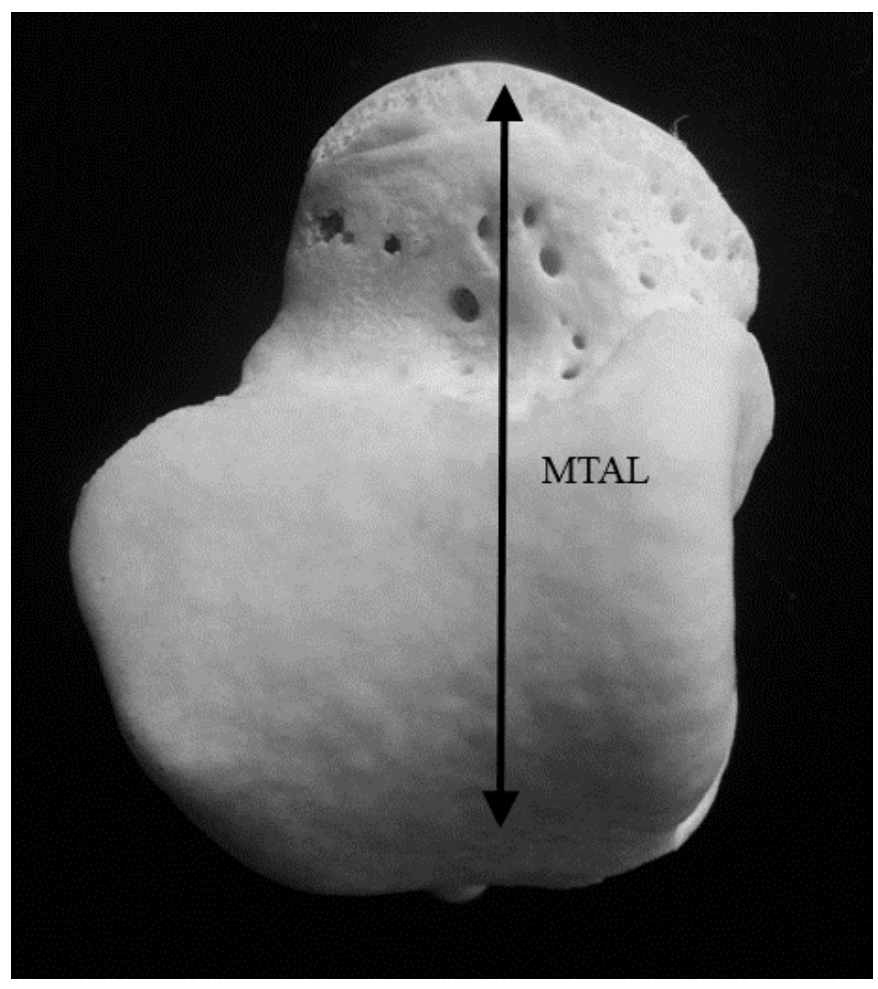

Figure 3.4 Superior view of a typical left talus depicting the Maximum Length of the Talus (MTAL) measurement variable. (Photo by Shelby Scott) 
All measurements were collected to the nearest $0.001 \mathrm{~mm}$ using a Vernier sliding caliper (Figure 3.5). The data were entered into a Microsoft Excel spreadsheet with males and females recorded separately.

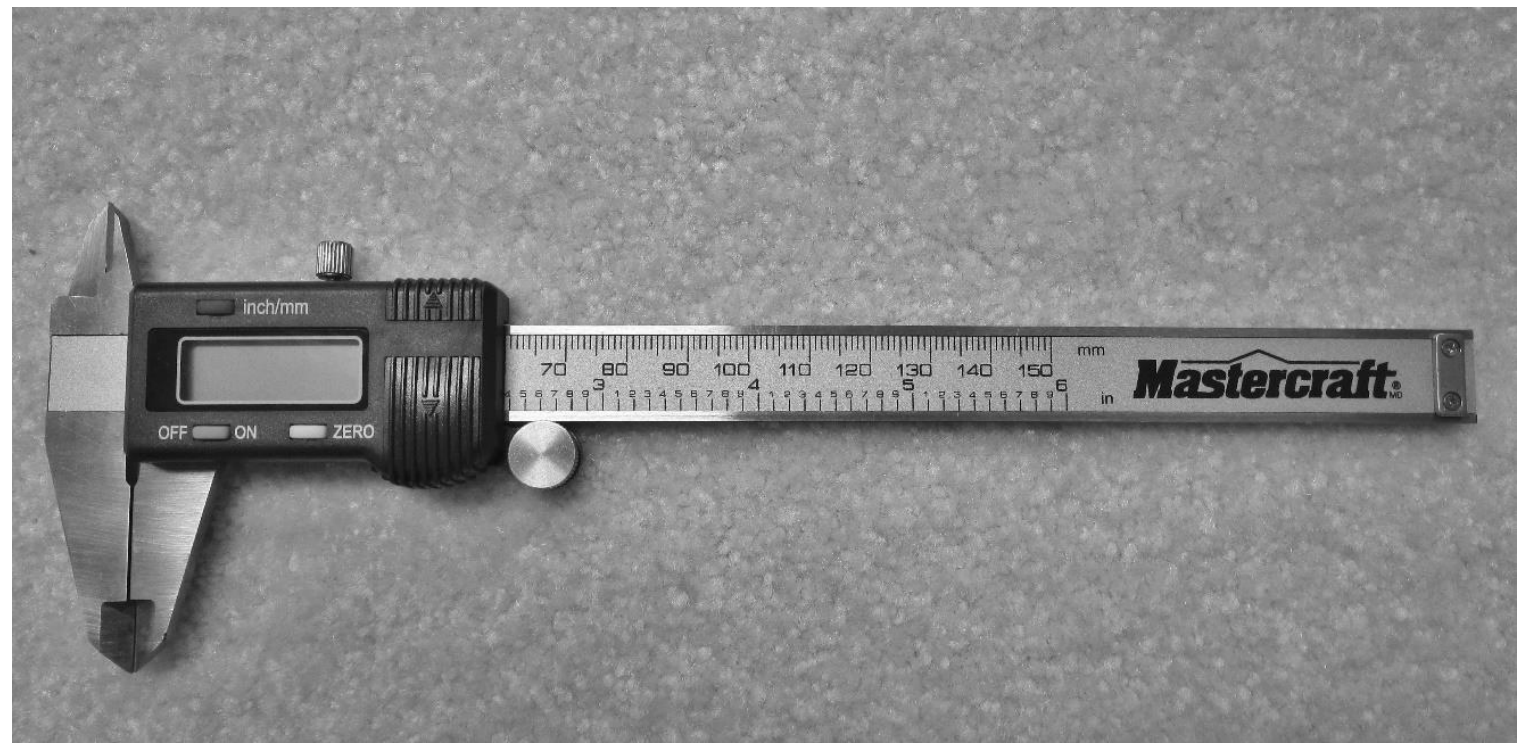

Figure 3.5 Mastercraft electronic (Vernier) caliper used for skeletal measurements. (Photo by Shelby Scott)

\subsection{Statistical Analyses}

\subsubsection{Normality}

Tests for normality were performed for each of the nine measurement variables of the calcaneus (Maximum Length, Maximum Height, Cuboidal Facet Height, Body Height, Minimum Breadth, Load Arm Length, Middle Breadth, Dorsal Articular Facet Breadth, and Dorsal Articular Facet Length) and one measurement variable of the talus (Maximum Length of the Talus) for both males and females separately. The KolmogorovSmirnov test for normality was utilized. Normality probability plots were created using Minitab version 17.0 statistical software in order to examine the distribution of data and 
highlight any potential outliers present in the dataset. A normal distribution is indicated when plotted measurement points display a linear distribution.

A statistical significance level of $5 \%$ error rate $(a=0.05)$ to measure normal data distribution was adjusted using a Bonferroni correction of $a=0.005$. This was completed by dividing the Bonferroni correction $(a=0.05)$ by the number of testable measurement variables $\left(\mathrm{N}_{\text {variables }}=10\right)$ to give a Bonferroni correction of $\mathrm{a}=0.005$. As the number of comparisons between measurement variables increases (i.e. the number of statistical tests), so does the likelihood that a Type 1 error may appear in the statistical outcome. A Bonferroni correction value is used to reduce the rate of Type 1 errors associated with multiple statistical comparisons. In the current research study, if the p-value is less than, or equal to, $0.005(p \leq 0.005)$ then the data are considered to be statistically not normal and non-parametric tests will be employed for further analyses. If the $\mathrm{p}$-value is greater than $0.005(p>0.005)$, then the data are considered to be normal and parametric tests will be employed for the statistical analyses.

\subsubsection{Descriptive Statistics}

Descriptive statistics were generated for each of the nine measurement variables of the calcaneus and the one variable of the talus. This was done separately for both males and females of the Thai population using Minitab version 17.0 statistical software. To examine the central tendency of the data, means were used as all data were determined to be statistically normal. The variability of the data was described using the maximum, minimum, and standard deviation. 


\subsubsection{Analysis of Side Differences in the Thai Population}

To determine if there was a statistically significant difference between the sides of the body, both left and right calcanei and left and right tali were measured using a randomized sub-sample of 60 individuals $\left(\mathrm{N}_{\text {total individuals }}=60 ; \mathrm{N}_{\text {males }}=30, \mathrm{~N}_{\text {females }}=30\right)$ from the Chiang Mai skeletal collection. Measurements were taken from both the left and right calcanei and tali for each individual. Therefore, four bones were examined for each individual. In total, 240 bones $\left(\mathrm{N}_{\text {total bones }}=240 ; \mathrm{N}_{\text {calcaneus }}=120, \mathrm{~N}_{\text {talus }}=120\right)$ were examined from 60 individuals $\left(\mathrm{N}_{\text {individuals }}=60\right)$. For each of the nine calcanei and one talar measurement variables, a paired t-test was performed using SPSS version 23.0 statistical software. This was done separately for males and females. There were no statistically significant differences between the left and right calcanei or the left and right tali $(p>$ 0.05). As there was no significant difference between the calcanei and tali of the left side of the body versus the calcanei and tali of the right side of the body, only left calcanei and left tali were measured following the protocol of Bidmos and Asala (2005). In circumstances where the left calcaneus and talus pair were not available due to them being absent, damaged, or affected by trauma or pathologies, the right calcanei and right tali pair were used and this was noted in the data collection spreadsheet.

\subsubsection{Analysis of Sexual Dimorphism in the Thai Population}

Two-sample t-tests were used in order to determine whether statistically significant differences existed between male and female calcaneal and talar measurement variables. In total, ten two-sample t-tests were completed using SPSS version 23.0 statistical software, i.e. one test for each of the nine measurement variables of the 
calcaneus and one test for the one measurement variable of the talus. A statistical significance level of $5 \%$ error rate $(a=0.05)$ is used in this research and was adjusted by dividing the Bonferroni correction $(a=0.05)$ by the number of testable measurement variables $\left(\mathrm{N}_{\text {variables }}=10\right)$ to give a Bonferroni correction of $a=0.005$. Values with a $p$ value of less than $0.005(p<0.005)$ indicate a statistically significant difference between the male and female calcaneal and talar measurements.

\subsubsection{Analysis of Observer Error and Reproducibility}

A number of different methods of statistical analyses have been suggested and used for testing precision and accuracy and evaluating error bias in scientific studies (Bidmos, 2006). The present study calculates intra-observer and inter-observer error rates using paired t-tests to evaluate error bias, following the protocol of Holland (1995), and also utilizes the concordance correlation of reproducibility method (Lin, 1989) following the protocol of Bidmos and Asala (2005). While these methods are similar in that they both measure precision and accuracy of the measuring technique, they are not quite the same. Intra-observer and inter-observer error take the difference between measurements, for each measurement variable, and compare the mean (average) difference to zero. This method is investigating overall deviations between repeated measurements. The concordance correlation of reproducibility method, however, investigates the agreement between paired measurements by measuring the variation from the concordance line, which is a $45^{\circ}$ line through the origin (i.e. the line one may see if all measurements were identical) (Lin, 1989; Meek, personal communication August 2017).

Intra-observer error and inter-observer error were analyzed, and were calculated separately for each of the nine measurement variables of the calcaneus and one 
measurement variable of the talus. Intra-observer error analyzes the differences between a single observer re-evaluating the same phenomenon, while inter-observer error analyzes the variation between two or more different individuals evaluating the same phenomenon. To ensure high accuracy and repeatability of the calcaneus and talus measurements, intraobserver and inter-observer error were calculated to ensure that measurements were free of researcher bias, measuring error, and gave similar results each time they were performed.

To test for intra-observer error, 60 skeletonized individuals $\left(\mathrm{N}_{\text {total individuals }}=60\right.$; $\mathrm{N}_{\text {males }}=30, \mathrm{~N}_{\text {females }}=30$ ) from the Chiang Mai skeletal collection were randomly selected. The left calcanei and left tali from each individual were re-measured by the current author one week after the initial measurements had been recorded. In total, 120 left foot bones $\left(\mathrm{N}_{\text {total bones }}=120 ; \mathrm{N}_{\text {calcaneus }}=60, \mathrm{~N}_{\text {talus }}=60\right)$ were examined from 60 individuals $\left(\mathrm{N}_{\text {individuals }}=60 ; \mathrm{N}_{\text {male }}=30, \mathrm{~N}_{\text {female }}=30\right)$. Buikstra and Ublelaker (1994) cite that a subsample group that is $20 \%$ of the original sample group is adequate for intra- and interobserver error analyses; the current sub-sample is large enough to allow accurate conclusions to be drawn about the original sample group. Each of the nine calcanei measurement variables and one talus measurement variable was re-measured and statistically analyzed using paired t-tests. A statistical significance level of $5 \%$ error rate $(a=0.05)$ is used in this research and was adjusted by dividing the Bonferroni correction $(a=0.05)$ by the number of testable measurement variables $\left(\mathrm{N}_{\text {variables }}=10\right)$ to give a Bonferroni correction of $a=0.005$. The measurements were considered to be repeatable, with no error bias, when the original data set and the re-measured sub-sample were compared and no significant difference $(p>0.005)$ was found. Each of the nine calcanei measurement variables and one talus measurement variable from this sample were also 
statistically analyzed using Lin's (1989) concordance correlation in order to evaluate intra-observer agreement. The measurements were considered to be repeatable and the measuring technique satisfactory, when the range of the concordance correlation coefficient obtained fell between the internationally accepted range of 0.90 to 0.98 (Cameron, 1984; Lin, 1989).

Inter-observer error was assessed at Chiang Mai University with the aid of a research assistant from the University of Central Lancashire (UCLan). The research assistant was provided with written descriptions and visual demonstrations on how to collect each measurement variable of the calcaneus and talus. She was instructed to record the measurement values for each measurement variable in a Microsoft Excel spreadsheet. A randomly selected sample of 60 skeletonized individuals $\left(\mathrm{N}_{\text {total individuals }}=\right.$ $\left.60 ; N_{\text {males }}=30, N_{\text {females }}=30\right)$ from the Chiang Mai skeletal collection were randomly selected and the left calcanei and left tali from each individual were re-measured and recorded into an Excel spreadsheet by the research assistant. In total, 120 left foot bones $\left(\mathrm{N}_{\text {total bones }}=120 ; \mathrm{N}_{\text {calcaneus }}=60, \mathrm{~N}_{\text {talus }}=60\right)$ were re-measured from 60 individuals $\left(\mathrm{N}_{\text {total }}\right.$ individuals $=60 ; \mathrm{N}_{\text {males }}=30, \mathrm{~N}_{\text {females }}=30$ ). Buikstra and Ublelaker (1994) cite that a subsample group that is $20 \%$ of the original sample group is adequate for intra- and interobserver error analyses; the current sub-sample is large enough to allow accurate conclusions to be drawn about the original sample group. Paired t-tests were used to examine statistical differences between the original data collected by the author and the data re-measured by the research assistant. A statistical significance level of 5\% error rate $(a=0.05)$ is used in this research, and was adjusted by dividing the Bonferroni correction $(a=0.05)$ by the number of testable variables $\left(\mathrm{N}_{\text {variables }}=10\right)$ to give a Bonferroni correction of $a=0.005$. The measurements were considered to be repeatable, and free of 
error bias, when the original data set and the re-measured sub-sample were compared, and no significant difference $(p>0.005)$ was found. Each of the nine calcanei measurement variables and one talus measurement variable from this sample were also statistically analyzed using Lin's (1989) concordance correlation in order to evaluate inter-observer agreement. The measurements were considered to be repeatable and the measuring technique satisfactory, when the range of the concordance correlation coefficient obtained fell between the internationally accepted range of 0.90 to 0.98 (Cameron, 1984; Lin, 1989).

\subsubsection{Simple and Multiple Regression Analyses}

The first objective of this research study is to investigate whether regression equations for the calcaneus, which were developed on a Black South African population (Bidmos and Asala, 2005) and regression equations for the talus, which were developed on a Black American population (Holland, 1995) can accurately predict the living stature of individuals from a Thai population.

There has been minimal research published on the estimation of living stature from skeletonized calcanei and tali using simple and multiple regression analyses. Methods of living stature estimation from the calcaneus have been developed only on a Black South African (Bidmos and Asala, 2005) and White South African (Bidmos, 2006) population. Methods of living stature estimation from the talus have been developed only on a Black American population (Holland, 1995). Pablos et al. (2013) investigated the relationship between living stature and maximum calcaneal and talar lengths in a Black and White American population using a robust regression method known as least trimmed 
squares (LTS). However, this method of statistical analyses is not commonly used in the forensic anthropology literature for the estimation of sex from skeletal remains and therefore cannot be compared with the current data (Bidmos and Asala, 2005; Bidmos, 2006; Holland, 1995). Furthermore, Zhang et al. (2016) investigated the relationship between living stature and measurement variables of the calcaneus in a Han Chinese population using computed radiography (CR) technology. Studies have shown that projection, landmark identification, and measurement errors may occur using radiographic imaging technologies such as computed radiography (CR), and that results from such studies may not be as accurate as those obtained using dry bone (Guenoun et al., 2012; Lagravère et al., 2010). As the current research study records calcaneal measurement variables from dry bone rather than computed radiography $(\mathrm{CR})$ technology, the Zhang et al. (2016) study cannot be compared with the current data. Therefore, the current project tests the Thai calcanei data with Black South African (Bidmos and Asala, 2005) and White South African (Bidmos, 2006) simple and multiple regression equations and tests the tali data with Black American (Holland, 1995) simple regression equations.

The simple and multiple regression equations of Bidmos and Asala (2005) provide an estimate of living stature. To use the simple and multiple regression equations created by Bidmos and Asala (2005) for a Black South African population (Table 3.4), the corresponding measurement variable value(s) from the Thai calcanei are multiplied by the appropriate coefficient and the product is added to the corresponding constant. 
Table 3.4 Regression equations (in $\mathrm{cm}$ ) for estimated skeletal height from the calcaneus of Black South Africans (Bidmos and Asala, 2005)

\begin{tabular}{|c|c|}
\hline \multicolumn{2}{|l|}{ Simple Regression Equations $*$} \\
\hline Male & Female \\
\hline $0.63($ MAXL $)+100.87$ & $0.82(\mathrm{MAXL})+82.49$ \\
\hline $0.75(\mathrm{MAXH})+118.67$ & $1.03(\mathrm{MAXH})+100.97$ \\
\hline $0.75(\mathrm{BH})+123.63$ & $1.08(\mathrm{BH})+105.81$ \\
\hline $0.51(\mathrm{MINB})+138.53$ & $1.17(\mathrm{MINB})+117.46$ \\
\hline $0.50(\mathrm{LAL})+128.47$ & $0.64(\mathrm{LAL})+115.80$ \\
\hline $1.07(\mathrm{MIDB})+105.67$ & $1.16(\mathrm{MIDB})+97.24$ \\
\hline $1.15(\mathrm{DAFB})+124.80$ & $1.54(\mathrm{DAFB})+110.72$ \\
\hline $0.96(\mathrm{DAFL})+122.48$ & $1.76(\mathrm{DAFL})+94.48$ \\
\hline \multicolumn{2}{|l|}{ Multiple Regression Equations* } \\
\hline Male & Female \\
\hline $\begin{array}{l}0.38(\mathrm{MAXL})+0.35(\mathrm{MAXH})+ \\
0.59(\mathrm{MIDB})+0.56(\mathrm{DAFB})+68.17\end{array}$ & $\begin{array}{l}0.69(\mathrm{MAXL})-0.67(\mathrm{LAL})+0.37(\mathrm{BH})+ \\
1.27(\mathrm{DAFL})+72.24\end{array}$ \\
\hline $\begin{array}{l}0.44(\mathrm{MAXL})+0.37(\mathrm{MAXH})+ \\
0.69(\mathrm{MIDB})+70.86\end{array}$ & $\begin{array}{l}0.81(\mathrm{MAXL})-0.73(\mathrm{LAL})+1.41(\mathrm{DAFL}) \\
+75.22\end{array}$ \\
\hline $0.46(\mathrm{MAXL})+0.85(\mathrm{MIDB})+78.37$ & $\begin{array}{l}0.30(\mathrm{MAXL})+0.51(\mathrm{MAXH})+ \\
1.16(\mathrm{DAFL})+68.11\end{array}$ \\
\hline $0.55(\mathrm{MAXL})+0.59(\mathrm{MAXH})+81.91$ & $0.71(\mathrm{MAXH})+1.43(\mathrm{DAFL})+74.88$ \\
\hline
\end{tabular}

$*(\mathrm{y} /$ predicted height $=)$

The outcome of the simple and multiple regression equations created by Bidmos and Asala (2005) is a preliminary estimation of skeletal height (in cm). Bidmos and Asala (2005) obtained estimates of skeletal height for each individual in their study from the sum of the following bone measurements: basibregmatic height of the skull, anterior body height of the second cervicle vertebra (C2) (including the odontoid process) to the fifth lumbar vertebrae (L5), the bicondylar (physiological) length of the femur, the condylomalleolar length of the tibia, and the articulated talocalcaneal height. This estimate of skeletal height does not however, take into account the thickness of the scalp, 
intervertebral discs, or the soft tissue of the sole of the foot (Bidmos and Asala, 2005) and is thus not reflective of an individual's living stature. To gain a final estimation of an individual's living stature, Bidmos and Asala (2005) add a soft tissue correction factor to the estimated skeletal height values generated from their simple and multiple regression equations, following the protocol of Fully (1956). In this way, the estimate of skeletal height, originally obtained through the simple and multiple regression equations of Bidmos and Asala (2005), is merely a first step in obtaining an estimate of living stature. With the addition of soft tissue correction factors, an estimate of living stature is obtained from the simple and multiple regression equations of Bidmos and Asala (2005).

For the calcaneus, eight simple regression equations and four multiple regression equations, created by Bidmos and Asala (2005) from a Black South African population, were applied to the Thai sample, and soft tissue correction factors were added to provide an estimate of living stature, and facilitate a comparison between estimated living stature and the total documented stature associated with individuals in the Thai sample. Males and females were analyzed separately. The Bidmos and Asala (2005) regression equations are shown in Table 3.4. A simple regression equation for the Cuboidal Facet Height $(\mathrm{CFH})$ measurement variable could not be tested on the Thai sample because it was not provided by Bidmos and Asala (2005), as it did not demonstrate a significant correlation in the Black South African population.

In contrast to the simple and multiple regression equations of Bidmos and Asala (2005), the simple regression equations created by Holland (1995) provide a value of estimated living stature (in $\mathrm{cm}$ ) from the talus and therefore no soft tissue correction factors are utilized when testing the equations of Holland (1995). To use the simple regression equations created by Holland (1995), the corresponding measurement variable 
(Maximum Length of the Talus) from the Thai tali is multiplied by the appropriate coefficient and the product is added to the corresponding constant. The final answer is a prediction of living stature (in $\mathrm{cm}$ ).

For the talus, one simple regression equation, created by Holland (1995) from a Black American population, was applied to the Thai sample to determine its accuracy for estimating the living stature of Thai individuals. Males and females were analyzed separately. The Holland (1995) regression equations are illustrated in Table 3.5.

Table 3.5 Regression equations (in $\mathrm{cm}$ ) for estimated living stature from the talus of Black Americans (Holland, 1995).

\begin{tabular}{|l|l|}
\hline \multicolumn{2}{|l|}{ Simple Regression Equations* } \\
\hline Male & Female \\
\hline $1.046(\mathrm{MTAL})+112.26$ & $1.046(\mathrm{MTAL})+97.55$ \\
\hline
\end{tabular}

$*(y /$ predicted living stature $=)$

From each simple regression equation and each multiple regression equation of the calcaneus and the talus, p-values and Pearson correlation values (" $r$-values", also known as correlation coefficients) were obtained. The p-value indicates if there is a statistically significant relationship between documented living stature and predicted living stature. In this research, a statistical significance level of $5 \%$ error rate $(a=0.05)$ is used and was adjusted by dividing the Bonferroni correction $(a=0.05)$ by the number of testable measurement variables $\left(\mathrm{N}_{\text {variables }}=10\right)$ to give a Bonferroni correction of $\mathrm{a}=$ 0.005. Therefore, if the $\mathrm{p}$-value is less than $0.005(p<0.005)$ then the relationship is statistically significant. The accuracy of each simple regression equation and each 
multiple regression equation of the calcaneus and the talus, generated from a Black South African population (Bidmos and Asala, 2005) and Black American (Holland, 1995) population and tested on the Thai sample data, was determined by the strength of the the correlation coefficient ( $r$-value). The correlation coefficient ( $r$-value) indicates the strength and direction of the relationship between measurement variables. Simple scatterplots were created to illustrate the strength of the relationship between documented living stature and the predicted living stature using the regression equations from Black South African (Bidmos and Asala, 2005) and Black American (Holland, 1995) populations. If the Black South African (Bidmos and Asala, 2005) and Black American (Holland, 1995) equations accurately estimated the living stature for Thai individuals, the scatterplot will display a linear pattern showing a statistically significant relationship between documented living stature and predicted living stature.

The second goal of this research was to develop population-specific regression equations for the calcaneus and the talus of the contemporary Thai population should the Black South African (Bidmos and Asala, 2005) and Black American (Holland, 1995) equations not accurately classify the individuals. To fulfill this goal, two sub-samples were created from the original Thai sample: Sample 1 and Sample 2. Sample 1 (main data set) was used in the derivation of regression equations for living stature estimation.

Sample 2 was an independent sample used to cross-validate the accuracy of the regression equations derived using Sample 1. Sample 2 was established by importing all the data into Minitab version 17.0 statistical software and generating a randomized sample consisting of paired bones from 60 left calcanei and 60 left tali $\left(\mathrm{N}_{\text {total bones }}=120 ; \mathrm{N}_{\text {calcaneus }}\right.$ $\left.=60, \mathrm{~N}_{\text {talus }}=60\right)$ from 60 individuals $\left(\mathrm{N}_{\text {individuals }}=60 ; \mathrm{N}_{\text {male }}=30, \mathrm{~N}_{\text {feamle }}=30\right)$. These 
individuals were excluded from the main data set, i.e. Sample 1, and formed an independent sample (Sample 2), which was used for cross-validation.

To develop regression equations from Sample 1, regression analyses were performed and equations were created using the unstandardized coefficient, constant values, and the Standard Error of the Estimate (SEE) for each equation. Simple regression analysis, in which individual measurement variables of the calcaneus and the talus were regressed against documented living stature to obtain simple regression equations, was performed. Nine simple regression equations were calculated for each measurement variable of the calcaneus following Bidmos and Asala (2005). One simple regression equation was calculated for the talar measurement variable following Holland (1995). This was done for males and females separately. Multiple regression analysis, in which combinations of these measurement variables were regressed against documented living stature to obtain multiple regression equations, was also performed. Using a direct method, a multiple equation was generated using all nine measurement variables of the calcaneus to determine whether accuracy rates are enhanced when utilizing all available measurement variables of the calcaneus. This was done for males and females separately. Multiple regression equations were also generated separately for males and females, using a stepwise method, in order to determine which combination of calcaneal measurement variables produced the most accurate estimation of living stature. To each multiple regression equation that was generated using measurement variables from the calcaneus, for both males and females, the talar measurement variable was added in order to create additional multiple regression equations. This was completed to investigate whether the addition of the talus measurement variable increased the accuracy rate for the estimation of living stature. 
The p-values, correlation coefficients ( $r$-values), f-statistics, and the Standard Error of the Estimate (SEE) were obtained for each simple and multiple regression equation generated following the protocol of Bidmos and Asala (2005). A statistical significance level of $5 \%$ error rate $(a=0.05)$ is used in this research and was adjusted by dividing the Bonferroni correction $(a=0.05)$ by the number of testable measurement variables $\left(\mathrm{N}_{\text {variables }}=10\right)$ to give a Bonferroni correction of $\mathrm{a}=0.005$. Therefore, if the $\mathrm{p}$ value is less than $0.005(\mathrm{p}<0.005)$ a statistically significant relationship exists. Overall, the accuracy of the estimation of stature (i.e. how close the estimate is to the documented living stature) for each simple and multiple regression equation is measured by the Standard Error of the Estimate (SEE); the lower the SEE as presented by a regression equation, the more accurate the estimate of stature.

Following the protocol of Bidmos and Asala (2005), ranges for living stature were obtained using the Standard Error of the Estimate (SEE) that was generated from each simple and each multiple regression equation created. This Standard Error of the Estimate (SEE) was added to, or subtracted from, the predicted living stature value to give the range for living stature.

\subsubsection{Cross-Validation of Thai Regression Equations}

An independent sample (Sample 2) was used to test the accuracy of the simple and multiple regression equations derived from Sample 1. Sample 2 consisted of paired tali from 60 left calcanei and 60 left tali $\left(\mathrm{N}_{\text {total bones }}=120 ; \mathrm{N}_{\text {calcaneus }}=60, \mathrm{~N}_{\text {talus }}=60\right)$ from 60 skeletonized individuals $\left(\mathrm{N}_{\text {individuals }}=60 ; \mathrm{N}_{\text {male }}=30, \mathrm{~N}_{\text {female }}=30\right)$. Simple and multiple regression equations that were derived from Sample 1 were tested on individuals from 
Sample 2 to give an estimate of living stature. Only those simple and multiple regression equations from Sample 1, which showed a statistically significant positive relationship with documented living stature (i.e. $\mathrm{p}<0.005$ ), were tested on Sample 2. Predicted living stature ranges were correlated with documented living stature using Minitab version 17.0 statistical software. Simple scatterplots were created to illustrate the strength of the relationship between documented living stature and the living stature predicted through cross-validation of the Thai simple and multiple regression equations. The p-values, correlation coefficients ( $r$-values), and the Standard Error of the Estimate (SEE) values were obtained. Following the protocol of Bidmos and Asala (2005) and Holland (1995), the accuracy of the estimation of living stature for each of the simple and multiple regression equations was assessed by calculating in what percent of cases, from Sample 2, did predicted living stature fall within 1 SEE and 2 SEE of the documented living stature.

\subsubsection{Population Comparison}

Measurements from the Thai population were compared to the Black South African (Bidmos and Asala, 2005), White South African (Bidmos, 2006), and Black American (Holland, 1995) populations using descriptive statistics. This was done for males and females, and for each measurement variables of the calcaneus and talus, separately. Two-sample t-tests were performed with Minitab version 17.0 statistical software in order to determine if there was a difference between the total number of individuals $(N)$, mean measurement values, and the standard deviations for each measurement variable of the calcaneus and talus between the Thai population and the Black South African (Bidmos and Asala, 2005), White South African (Bidmos, 2006), and Black American (Holland, 1995) populations. 


\section{CHAPTER 4: RESULTS}

\section{1 $\quad$ Research Objectives}

The current research study focuses on nine measurements of the calcaneus (Maximum Length, Maximum Height, Cuboidal Facet Height, Body Height, Minimum Breadth, Load Arm Length, Middle Breadth, Dorsal Articular Facet Breadth, and Dorsal Articular Facet Length) and one measurement of the talus (Maximum Length of the Talus) to establish an accurate method of living stature estimation for a contemporary Thai population. The objectives of this research are to:

1. investigate whether regression equations for the calcaneus, which were developed on a Black South African population (Bidmos and Asala, 2005), and regression equations for the talus, which were developed on a Black American population (Holland, 1995) can accurately predict the living stature of individuals from a contemporary Thai population.

2. develop population-specific simple regression and multiple regression equations for the calcaneus and the talus of the contemporary Thai population should the Black South African (Bidmos and Asala, 2005) and Black American (Holland, 1995) equations not accurately predict the living stature of the Thai individuals.

3. compare the results from the contemporary Thai population to other populations.

\subsection{Normality}

Normality was assessed for each of the nine calcaneal measurement variables (Maximum Length, Maximum Height, Cuboidal Facet Height, Body Height, Minimum Breadth, Load Arm Length, Middle Breadth, Dorsal Articular Facet Breadth, and Dorsal 
Articular Facet Length) and one talar measurement variable (Maximum Length of the Talus) using Minitab version 17.0 statistical software. Males and females were independently assessed resulting in a total of 20 variables $\left(\mathrm{N}_{\text {total variables }}=20 ; \mathrm{N}_{\text {male variables }}=\right.$ $10, \mathrm{~N}_{\text {female variables }}=10$ ). Table 4.1 shows the results of the calculated normality probability for calcaneal and talar measurement variables for both males and females. Due to the large number of measurement variables being analyzed, a statistical significance level of $5 \%$ error rate $(a=0.05)$ was adjusted using a Bonferroni correction of $a=0.005$ in order to account for type- 1 error. If the $\mathrm{p}$-value is less than, or equal to, $0.005(p \leq 0.005)$, then the data are considered to be not normally distributed. If the p-value is greater than 0.005 $(p>0.005)$ then the data are considered to be normally distributed. The results show that the male and female data were normally distributed for all calcaneal and talar measurement variables. As all data were normally distributed, statistical analyses proceeded using parametric tests (i.e. tests for normally distributed data). 
Table 4.1 Normality probability p-values assessed in males and females.

\begin{tabular}{lll}
\hline Measurement variable & $\begin{array}{l}p \text {-value } \\
\text { (Male) }\end{array}$ & $\begin{array}{l}p \text {-value } \\
\text { (Female) }\end{array}$ \\
\hline Calcaneus & & \\
\hline & $>0.150$ & 0.075 \\
MAXL & $>0.150$ & $>0.150$ \\
MAXH & $>0.150$ & 0.088 \\
CFH & $>0.150$ & 0.050 \\
BH & $>0.150$ & $>0.150$ \\
MINB & $>0.150$ & 0.070 \\
LAL & $>0.150$ & $>0.150$ \\
MIDB & 0.077 & $>0.150$ \\
DAFB & $>0.150$ & $>0.150$ \\
DAFL & & \\
& & \\
\hline Talus & & \\
\hline & & \\
MTAL & & \\
\hline
\end{tabular}

Normality probability plots were created for males and females for each calcaneal and talar measurement variable in order to allow for a visual observation of the data distribution and to highlight any potential outliers present in the dataset. If the data exhibit a linear pattern, then the data are normally distributed. For the current research, all normality probability plots indicated normally distributed data with no significant outliers present in the dataset. Figure 4.1 is an example of a probability plot for the male Maximum Length (MAXL) calcaneal measurement variable. Refer to Appendix C for the probability plots of each calcaneal and talar measurement variable. 


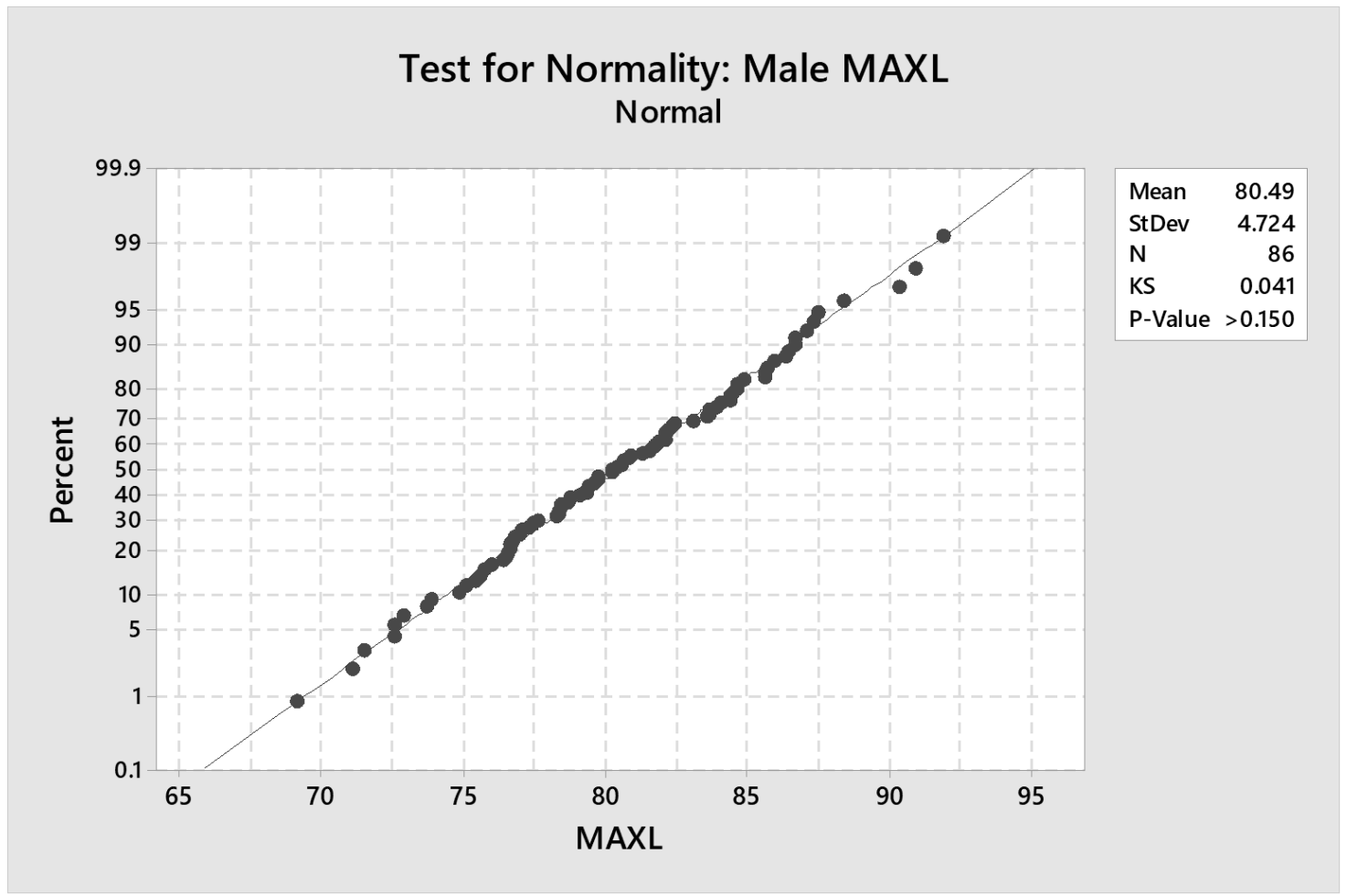

Figure 4.1 Normality probability plot for the male Maximum Length (MAXL) calcaneal measurement variable.

\subsection{Descriptive Statistics}

The calcaneus and the talus from 233 skeletonized individuals $\left(\mathrm{N}_{\text {total individuals }}=\right.$ $233 ; \mathrm{N}_{\text {males }}=116, \mathrm{~N}_{\text {females }}=117$ ) of Thai ancestry were examined for their potential in estimating living stature. In total, 466 individual calcanei and tali $\left(\mathrm{N}_{\text {total bones }}=466\right.$; $\mathrm{N}_{\text {calcaneus }}=233, \mathrm{~N}_{\text {talus }}=233$ ) were studied from the Chiang Mai skeletal collection in Chiang Mai, Thailand. Nine measurements of the calcaneus were collected: Maximum Length, Maximum Height, Cuboidal Facet Height, Body Height, Minimum Breadth, Load Arm Length, Middle Breadth, Dorsal Articular Facet Breadth, and Dorsal Articular Facet Length. This method follows the protocol of Bidmos and Asala (2005). One measurement of the talus was collected (Maximum Length of the Talus), following the protocol of Holland (1995). 
Table 4.2 shows the descriptive statistics for males and females for each of the nine calcaneal measurement variables and the one talar measurement variable. This table illustrates the number $(N)$ of calcanei or tali assessed for each measurement variable, as well as maximum measurement length (Maximum), minimum measurement length (Minimum), mean, and standard deviation (SD). These measurement values are shown in millimeters $(\mathrm{mm})$. 
Table 4.2 Descriptive statistics for calcaneal and talar measurement variables for both males and females.

\begin{tabular}{lcccll}
\hline $\begin{array}{l}\text { Measurement } \\
\text { variable }\end{array}$ & $N$ & $\begin{array}{l}\text { Mean } \\
(\mathrm{mm})\end{array}$ & $\begin{array}{l}\text { SD } \\
(\mathrm{mm})\end{array}$ & $\begin{array}{l}\text { Minimum Length } \\
(\mathrm{mm})\end{array}$ & $\begin{array}{l}\text { Maximum Length } \\
(\mathrm{mm})\end{array}$ \\
\hline Calcaneus & & & & & \\
\hline Male & & & & & \\
& & & & & 91.86 \\
MAXL & 86 & 80.49 & 4.72 & 69.11 & 51.98 \\
MAXH & 86 & 43.62 & 3.56 & 31.32 & 32.29 \\
CFH & 86 & 26.79 & 2.19 & 21.69 & 49.14 \\
BH & 86 & 39.06 & 2.91 & 32.45 & 30.26 \\
MINB & 86 & 25.71 & 1.88 & 21.55 & 57.35 \\
LAL & 86 & 48.68 & 2.82 & 42.32 & 36.57 \\
MIDB & 86 & 40.97 & 2.21 & 35.10 & 33.90 \\
DAFB & 86 & 29.37 & 1.88 & 24.56 & \\
DAFL & 86 & 29.25 & 1.95 & 22.13 &
\end{tabular}

Female

$\begin{array}{llllll}\text { MAXL } & 87 & 73.44 & 4.26 & 66.47 & 84.05 \\ \text { MAXH } & 87 & 39.53 & 3.57 & 27.24 & 50.16 \\ \text { CFH } & 87 & 24.29 & 1.82 & 21.15 & 28.71 \\ \text { BH } & 87 & 34.29 & 2.45 & 29.03 & 41.37 \\ \text { MINB } & 87 & 23.48 & 1.90 & 19.37 & 30.70 \\ \text { LAL } & 87 & 44.15 & 2.48 & 38.16 & 49.40 \\ \text { MIDB } & 87 & 37.08 & 2.16 & 31.93 & 44.14 \\ \text { DAFB } & 87 & 26.02 & 1.76 & 21.83 & 30.96 \\ \text { DAFL } & 87 & 25.80 & 1.78 & 21.54 & 31.66\end{array}$

\section{Talus}

Male

MTAL

$\begin{array}{llll}86 & 56.38 & 3.52 & 48.20\end{array}$

67.08

Female

$\begin{array}{llllll}\text { MTAL } & 87 & 50.02 & 3.13 & 44.22 & 59.27\end{array}$




\subsection{Analysis of Sexual Dimorphism in the Thai Population}

Each of the nine calcaneal measurement variables and the one talar measurement variable were tested to assess whether statistically significant sexually dimorphic differences existed between male and female calcanei, as well as between male and female tali, in the Chiang Mai skeletal collection. The data were normally distributed, which allowed for the use of two-sample t-tests. Due to the large number of measurement variables being analyzed $\left(\mathrm{N}_{\text {variables }}=10\right)$ a statistical significance level of $5 \%$ error rate $(\mathrm{a}$ $=0.05)$ was adjusted using a Bonferroni correction of $a=0.005$. Therefore, if a

measurement variable had a $\mathrm{p}$-value that was less than, or equal to, $0.005(p \leq 0.005)$, this indicated a statistically significant difference between the male and female calcaneal and talar measurement values (Table 4.3). The results of the statistical analyses indicated that all calcaneal and talar measurement variables were sexually dimorphic within this population $(p<0.005)$. 
Table 4.3 Two-sample t-tests evaluating sexual dimorphism of males and females for each calcaneal and talar measurement variable.

\begin{tabular}{lllll}
\hline Measurement variable & Male $(N)$ & Female $(N)$ & $t$-value & $p$-value \\
\hline Calcaneus & & & & \\
\hline & & & & \\
MAXL & 86 & 87 & 10.32 & $0.00^{*}$ \\
MAXH & 86 & 87 & 7.55 & $0.00^{*}$ \\
CFH & 86 & 87 & 8.17 & $0.00^{*}$ \\
BH & 86 & 87 & 11.68 & $0.00^{*}$ \\
MINB & 86 & 87 & 7.76 & $0.00^{*}$ \\
LAL & 86 & 87 & 11.23 & $0.00^{*}$ \\
MIDB & 86 & 87 & 11.72 & $0.00^{*}$ \\
DAFB & 86 & 87 & 12.12 & $0.00^{*}$ \\
DAFL & 86 & 87 & 12.21 & $0.00^{*}$ \\
Talus & & & & \\
\hline & & & & \\
MTAL & 86 & 87 & 12.56 & $0.00^{*}$ \\
\hline
\end{tabular}

* Significant difference at $p<0.005$

\subsection{Intra- and Inter-Observer Error}

Intra-observer and inter-observer error were evaluated separately for each of the nine calcaneal measurement variables (Maximum Length, Maximum Height, Cuboidal Facet Height, Body Height, Minimum Breadth, Load Arm Length, Middle Breadth, Dorsal Articular Facet Breadth, and Dorsal Articular Facet Length) and the one talar measurement variable (Maximum Length of the Talus) to measure the repeatability and accuracy of the calcaneal and talar measurements. Intra-observer error evaluates differences between measurements collected by a single individual on the same sample. Intra- observer error was assessed by the current author by re-measuring a randomly selected sample of 60 skeletonized individuals $\left(\mathrm{N}_{\text {total individuals }}=60 ; \mathrm{N}_{\text {males }}=30, \mathrm{~N}_{\text {females }}=\right.$ 30) consisting of 60 left calcanei and 60 left tali $\left(\mathrm{N}_{\text {total bones }}=120 ; \mathrm{N}_{\text {calcaneus }}=60, \mathrm{~N}_{\text {talus }}=\right.$ 
60) from the Chiang Mai skeletal collection. Each of the nine calcaneal measurement variables and the one talar measurement variable were re-measured by the current author. The data were normally distributed and, therefore, statistically analyzed using paired ttests and the concordance correlation of reproducibility method (Lin, 1989). This was done for males and females separately.

A statistical significance level of $5 \%$ error rate $(a=0.05)$ is used in this research and was adjusted by dividing the Bonferroni correction $(a=0.05)$ by the number of testable variables $\left(\mathrm{N}_{\text {variables }}=10\right)$ to give a Bonferroni correction of $\mathrm{a}=0.005$. The measurements were considered to be repeatable, with no error bias, when the original data set and the re-measured sub-sample were compared and no significant difference $(p \geq$ 0.005) was found. The resulting p-values showed no statistically significant difference between the original sample and the re-measured sub-sample $(p>0.005)$. This indicates that each of the nine calcaneal measurements and one talar measurement were accurately repeated for both males and females. As well, each of the nine calcanei measurement variables and one talus measurement variable from the Thai sample were statistically analyzed using Lin's (1989) concordance correlation in order to evaluate intra-observer agreement. Each measurement variable of the calcaneus and the talus was considered to be repeatable if the values of concordance correlation coefficients fell within the internationally accepted standard range of 0.90 to 0.99 (Cameron, 1984; Lin, 1989). Of all the calcaneal and talar measurement variables, only the male DAFB (0.742) measurement and the female CFH (0.806), BH (0.896), MINB (0.819), DAFB (0.813) measurements did not fall within the internationally accepted standard range of 0.90 to 0.99 (Cameron, 1984; Lin, 1989). Table 4.4 shows the results of the statistical analyses performed for assessing intra-observer error rates for both the calcaneus and the talus. 
Table 4.4 Statistical analyses of the intra-observer error rates and concordance correlation coefficients of reproducibility ( $\mathrm{Rc}$ ) for the calcaneus and talus of individuals in the Chiang Mai skeletal collection.

\begin{tabular}{lclll}
\hline Measurement & $n$ & $\begin{array}{l}\text { Test } \\
\text { statistic }\end{array}$ & $p$-value & $R c$-value \\
Calcaneus & & & \\
\hline Male & & & & \\
& & & & \\
MAXL & 30 & -0.307 & 0.761 & 0.978 \\
MAXH & 30 & 0.139 & 0.752 & 0.956 \\
CFH & 30 & -1.599 & 0.121 & 0.958 \\
BH & 30 & -0.743 & 0.464 & 0.957 \\
MINB & 30 & -0.006 & 0.995 & 0.927 \\
LAL & 30 & -1.391 & 0.175 & 0.961 \\
MIDB & 30 & -0.206 & 0.838 & 0.933 \\
DAFB & 30 & 1.661 & 0.108 & 0.742 \\
DAFL & 30 & -0.930 & 0.360 & 0.909
\end{tabular}

Female

$\begin{array}{lllll}\text { MAXL } & 30 & 0.869 & 0.392 & 0.997 \\ \text { MAXH } & 30 & -1.976 & 0.058 & 0.971 \\ \text { CFH } & 30 & -1.413 & 0.168 & 0.806 \\ \text { BH } & 30 & -0.705 & 0.487 & 0.896 \\ \text { MINB } & 30 & -1.027 & 0.313 & 0.819 \\ \text { LAL } & 30 & -1.177 & 0.249 & 0.982 \\ \text { MIDB } & 30 & -1.559 & 0.130 & 0.963 \\ \text { DAFB } & 30 & 0.159 & 0.875 & 0.813 \\ \text { DAFL } & 30 & -1.002 & 0.324 & 0.923\end{array}$

\section{Talus}

Male

MTAL

$30-1.726$

0.095

0.982

Female

MTAL

$30-1.879$

0.070

0.937 
Inter-observer error evaluates potential differences between measurements that are collected by two or more individuals on the same sample. Inter- observer error was assessed with the aid of a research assistant from the University of Central Lancashire (UCLan). A randomly selected sample of 60 skeletonized individuals $\left(\mathrm{N}_{\text {total individuals }}=60\right.$; $\left.\mathrm{N}_{\text {males }}=30, \mathrm{~N}_{\text {females }}=30\right)$ consisting of 60 left calcanei and 60 left tali $\left(\mathrm{N}_{\text {total bones }}=120\right.$; $\left.N_{\text {calcaneus }}=60, N_{\text {talus }}=60\right)$ was used to evaluate inter-observer error. Each of the nine calcaneal measurement variables and the one talar measurement variable were remeasured and recorded into an Excel spreadsheet by the research assistant. This was done separately for males and females. The data were normally distributed and, therefore, paired t-tests were used to examine statistical differences between the original data collected by the current author and the data re-measured by the research assistant.

A statistical significance level of 5\% error rate $(a=0.05)$ was used in this research and was adjusted by dividing the Bonferroni correction $(a=0.05)$ by the number of testable variables $\left(\mathrm{N}_{\text {variables }}=10\right)$ to give a Bonferroni correction of $\mathrm{a}=0.005$. The measurements were considered to be repeatable, and free of error bias, when there was no significant difference $(p>0.005)$ when the original dataset and the re-measured subsample were compared. The resulting p-values showed no statistically significant difference in males, or in females, between the original sample and the re-measured sample. This indicates that all measurements were accurately repeated for both males and females between researchers. As well, each of the nine calcanei measurement variables and one talus measurement variable from the Thai sample were statistically analyzed using Lin's (1989) concordance correlation in order to evaluate inter-observer agreement. Each measurement variable of the calcaneus and the talus was considered to be repeatable if the values of concordance correlation coefficients fell within the internationally 
accepted standard range of 0.90 to 0.99 (Cameron, 1984; Lin, 1989). Of all the calcaneal and talar measurement variables, only the male DAFB (0.796) measurement, and the female BH (0.659) and MINB (0.896) measurements did not fall within the internationally accepted standard range of 0.90 to 0.99 (Cameron, 1984; Lin, 1989). Table 4.5 shows the results of the statistical analyses performed for assessing inter-observer error for both the calcaneus and the talus. 
Table 4.5 Statistical analyses of the inter-observer error rates and concordance correlation coefficients of reproducibility ( $\mathrm{Rc}$ ) for the calcaneus and talus of individuals in the Chiang Mai skeletal collection.

\begin{tabular}{lclll}
\hline Measurement & $n$ & $\begin{array}{l}\text { Test } \\
\text { statistic }\end{array}$ & $p$-value & $R c$-value \\
& & & \\
\hline Calcaneus & & & & \\
\hline Male & & & & \\
& & & & \\
MAXL & 30 & 1.255 & 0.220 & 0.999 \\
MAXH & 30 & 1.065 & 0.296 & 0.989 \\
CFH & 30 & -1.993 & 0.056 & 0.994 \\
BH & 30 & -1.354 & 0.186 & 0.987 \\
MINB & 30 & 0.012 & 0.991 & 0.987 \\
LAL & 30 & -1.483 & 0.149 & 0.992 \\
MIDB & 30 & -0.517 & 0.609 & 0.986 \\
DAFB & 30 & 0.272 & 0.788 & 0.769 \\
DAFL & 30 & -1.597 & 0.121 & 0.987
\end{tabular}

Female

$\begin{array}{lllll}\text { MAXL } & 30 & -0.219 & 0.829 & 0.990 \\ \text { MAXH } & 30 & -0.465 & 0.645 & 0.997 \\ \text { CFH } & 30 & -1.442 & 0.160 & 0.999 \\ \text { BH } & 30 & -0.219 & 0.828 & 0.659 \\ \text { MINB } & 30 & 0.358 & 0.723 & 0.896 \\ \text { LAL } & 30 & -1.898 & 0.068 & 0.994 \\ \text { MIDB } & 30 & -0.111 & 0.912 & 0.971 \\ \text { DAFB } & 30 & -0.670 & 0.508 & 0.937 \\ \text { DAFL } & 30 & -1.079 & 0.289 & 0.989\end{array}$

\begin{tabular}{lllll}
\hline Talus & & & & \\
\hline Male & & & & \\
MTAL & 30 & -1.897 & 0.068 & 0.915 \\
Female & & & & \\
MTAL & 30 & 0.496 & 0.623 & 0.999 \\
\hline
\end{tabular}




\subsection{Simple and Multiple Regression Analyses}

\subsubsection{Accuracy of the Bidmos and Asala (2005) Calcaneal Regression Equations}

The first objective of the current research was to investigate whether regression equations for the calcaneus, which were developed on a Black South African population (Bidmos and Asala, 2005), and regression equations for the talus, which were developed on a Black American population (Holland, 1995), can accurately predict the living stature of individuals from a Thai population. To fulfill this objective, each of the simple and multiple regression equations for the calcaneus that were generated from a Black South African population (Bidmos and Asala, 2005) were tested on the Thai sample data. However, simple regression equations using the Cuboidal Facet Height (CFH) calcaneal measurement variable could not be tested on the Thai sample data because Bidmos and Asala (2005) do not provide simple regression equations that use the CFH calcaneal measurement variable. The accuracy of each calcaneal simple and multiple regression equation that were generated from a Black South African population (Bidmos and Asala, 2005) and tested on the Thai sample data is determined by the strength of the Pearson correlation value ( $r$-value), also known as the correlation coefficient ( $r$-value). The correlation coefficient ( $r$-value) indicates the strength and direction of the relationship between measurement variables.

Table 4.6 shows the results of the male Black South African calcaneal regression equations (Bidmos and Asala, 2005) when applied to the Thai population. The regression equations are shown in decreasing order of the value (strength) of the correlation coefficient. A Bonferroni correction of $a=0.005$ is used in this research study. For Thai males, with the exception of the Dorsal Articular Facet Breadth (DAFB) and Dorsal 
Articular Facet Length (DAFL), all simple regression equations of the calcaneus have a pvalue less than $0.005(p<0.005)$.

Table 4.6 Accuracies of the male Black South African (Bidmos and Asala, 2005) regression equations for the calcaneus when applied to the Thai population.

\begin{tabular}{lll}
\hline Simple Regression Equations ** & $\begin{array}{l}\text { Correlation } \\
(r \text {-value })\end{array}$ & $p$-value \\
& 0.63 & $0.000^{*}$ \\
$0.63(\mathrm{MAXL})+100.87$ & 0.49 & $0.000^{*}$ \\
$0.50(\mathrm{LAL})+128.47$ & 0.48 & $0.000^{*}$ \\
$0.51(\mathrm{MINB})+138.53$ & 0.47 & $0.000^{*}$ \\
$0.75(\mathrm{BH})+123.63$ & 0.46 & $0.000^{*}$ \\
$0.75(\mathrm{MAXH})+118.67$ & 0.42 & $0.000^{*}$ \\
$1.07(\mathrm{MIDB})+105.67$ & 0.28 & 0.011 \\
$1.15(\mathrm{DAFB})+124.80$ & 0.28 & 0.010 \\
$0.96(\mathrm{DAFL})+122.48$ & & \\
\hline Multiple Regression Equations ** & & $0.000^{*}$ \\
\hline $0.55(\mathrm{MAXL})+0.59(\mathrm{MAXH})+81.91$ & 0.61 & \\
\hline $0.44(\mathrm{MAXL})+0.37(\mathrm{MAXH})+0.69(\mathrm{MIDB})+70.86$ & 0.63 & $0.000^{*}$ \\
\hline & & \\
\hline
\end{tabular}

$* \mathrm{p}<0.005$

$* *(\mathrm{y} /$ predicted living stature $=)$ 
This means that for each calcaneal measurement variable, excluding DAFB and DAFL, there was a statistically significant relationship between the documented living stature and the predicted living stature for each Thai male using the male Black South African (Bidmos and Asala, 2005) regression equations for the calcaneus. Of the male calcaneal regression equations that show a statistically significant relationship $(p<$ 0.005), the simple regression equation using the Maximum Length (MAXL) calcaneal measurement variable and the multiple regression equation using the Maximum Length (MAXL), Maximum Height (MAXH), and Middle Breadth (MIDB) calcaneal measurement variables show the strongest relationship between documented living stature and predicted living stature $(r=0.63)$. Overall, the male Black South African (Bidmos and Asala, 2005) multiple regression equations for the calcaneus demonstrate a stronger relationship (i.e. higher correlation coefficients) than the male Black South African (Bidmos and Asala, 2005) simple regression equations for the calcaneus, indicating that they are better predictors of living stature for males in the Thai population.

Table 4.7 shows the results of the female Black South African (Bidmos and Asala, 2005) calcaneal regression equations when applied to the Thai population. The regression equations are shown in decreasing order of the value (strength) of the correlation coefficient. A Bonferroni correction of $a=0.005$ is used in this research. For females, with the exception of the Minimum Breadth (MINB) equation, all simple regression equations for the calcaneus have a p-value less than $0.005(p<0.005)$. This means that for all calcaneal measurement variables, excluding MINB, there was a statistically significant relationship between the documented living stature and the predicted living stature for each Thai female using the female Black South African (Bidmos and Asala, 2005) simple regression equations. Of the female regression equations for the calcaneus 
that show a statistically significant relationship $(p<0.005)$, the simple regression equation using the Maximum Length (MAXL) calcaneal measurement variable and the multiple regression equation using the Maximum Length (MAXL), Maximum Height (MAXH), and Dorsal Articular Facet Length (DAFL) calcaneal measurement variables show the strongest relationship between documented living stature and predicted living stature $(r=0.54)$. Overall, the female Black South African (Bidmos and Asala, 2005) multiple regression equations for the calcaneus demonstrate a stronger relationship (i.e. higher correlation coefficients) than the female Black South African (Bidmos and Asala, 2005) simple regression equations for the calcaneus, indicating that they are better predictors of living stature for females in the Thai population. 
Table 4.7 Accuracies of the female Black South African (Bidmos and Asala, 2005) regression equations for the calcaneus when applied to the Thai population.

\begin{tabular}{|c|c|c|}
\hline Simple Regression Equations** & $\begin{array}{l}\text { Correlation } \\
\text { ( } r \text {-value) }\end{array}$ & $p$-value \\
\hline $0.82($ MAXL) + 82.49 & 0.54 & $0.000^{*}$ \\
\hline $0.64(\mathrm{LAL})+115.80$ & 0.53 & $0.000 *$ \\
\hline $1.03(\mathrm{MAXH})+100.97$ & 0.47 & $0.000 *$ \\
\hline $1.08(\mathrm{BH})+105.81$ & 0.41 & $0.000 *$ \\
\hline $1.54(\mathrm{DAFB})+110.72$ & 0.40 & $0.000 *$ \\
\hline $1.76(\mathrm{DAFL})+94.48$ & 0.39 & $0.000^{*}$ \\
\hline $1.16(\mathrm{MIDB})+97.24$ & 0.35 & $0.001 *$ \\
\hline $1.17(\mathrm{MINB})+117.46$ & 0.23 & 0.034 \\
\hline \multicolumn{3}{|l|}{ Multiple Regression Equations** } \\
\hline $0.30(\mathrm{MAXL})+0.51(\mathrm{MAXH})+1.16(\mathrm{DAFL})+68.11$ & 0.54 & $0.000 *$ \\
\hline $\begin{array}{l}0.69(\mathrm{MAXL})-0.67(\mathrm{LAL})+0.37(\mathrm{BH})+1.27(\mathrm{DAFL})+ \\
72.24\end{array}$ & 0.49 & $0.000 *$ \\
\hline $0.71(\mathrm{MAXH})+1.43(\mathrm{DAFL})+74.88$ & 0.49 & $0.000 *$ \\
\hline $0.81(\mathrm{MAXL})-0.73(\mathrm{LAL})+1.41(\mathrm{DAFL})+75.22$ & 0.47 & $0.000^{*}$ \\
\hline
\end{tabular}

Overall, for both males and females, the Black South African (Bidmos and Asala, 2005) MAXL simple regression equation for the calcaneus displays the highest correlation coefficient value (and is thus the most accurate for stature estimation in a Thai population) between documented living stature and predicted living stature for Thai individuals ( $r=0.63$ in males, $r=0.54$ in females). Documented living stature and predicted living stature, when using these calcaneal regression equations, show a statistically significant relationship $(p<0.005)$. However, the correlation value $(r$-value) indicates that this relationship is not strong ( $r=0.63$ in males, $r=0.54$ in females). 
Similarly, the multiple regression equations for the calcaneus (for both Thai males and Thai females), have r-values ranging from 0.47 to 0.63 .

The strength and direction of the relationship between documented living stature and the predicted living stature, when using the Black South African (Bidmos and Asala, 2005) calcaneal regression equations, was illustrated through simple scatterplots. A scatterplot is a figure that assists in the visualization of a relationship between two variables in two-dimensional space. Simple scatterplots were created to analyze the correlation between measurement variables for each Black South African (Bidmos and Asala, 2005) simple and multiple regression equation for the calcaneus that was tested on the Thai sample data. A strong relationship between variables is depicted in a scatterplot as a tight cluster in which data points are close together and show a clear linear relationship. The correlation coefficient is a numerical indication of the strength of this relationship of linearity. Therefore, while the majority of the simple and multiple regression equations for the calcaneus that were developed from the Black South African population (Bidmos and Asala, 2005) show a statistically significant relationship with the documented living statures of the Thai individuals, these relationships are not strong and are not accurate when estimating stature in a Thai population. Figure 4.2 is an example of a simple scatterplot when the male Black South African Maximum Length (MAXL) simple regression equation (Bidmos and Asala, 2005) is applied to the Thai sample data. Appendix D shows simple scatterplots when male and female Black South African (Bidmos and Asala, 2005) calcaneal simple and multiple regression equations are applied to the Thai population. 


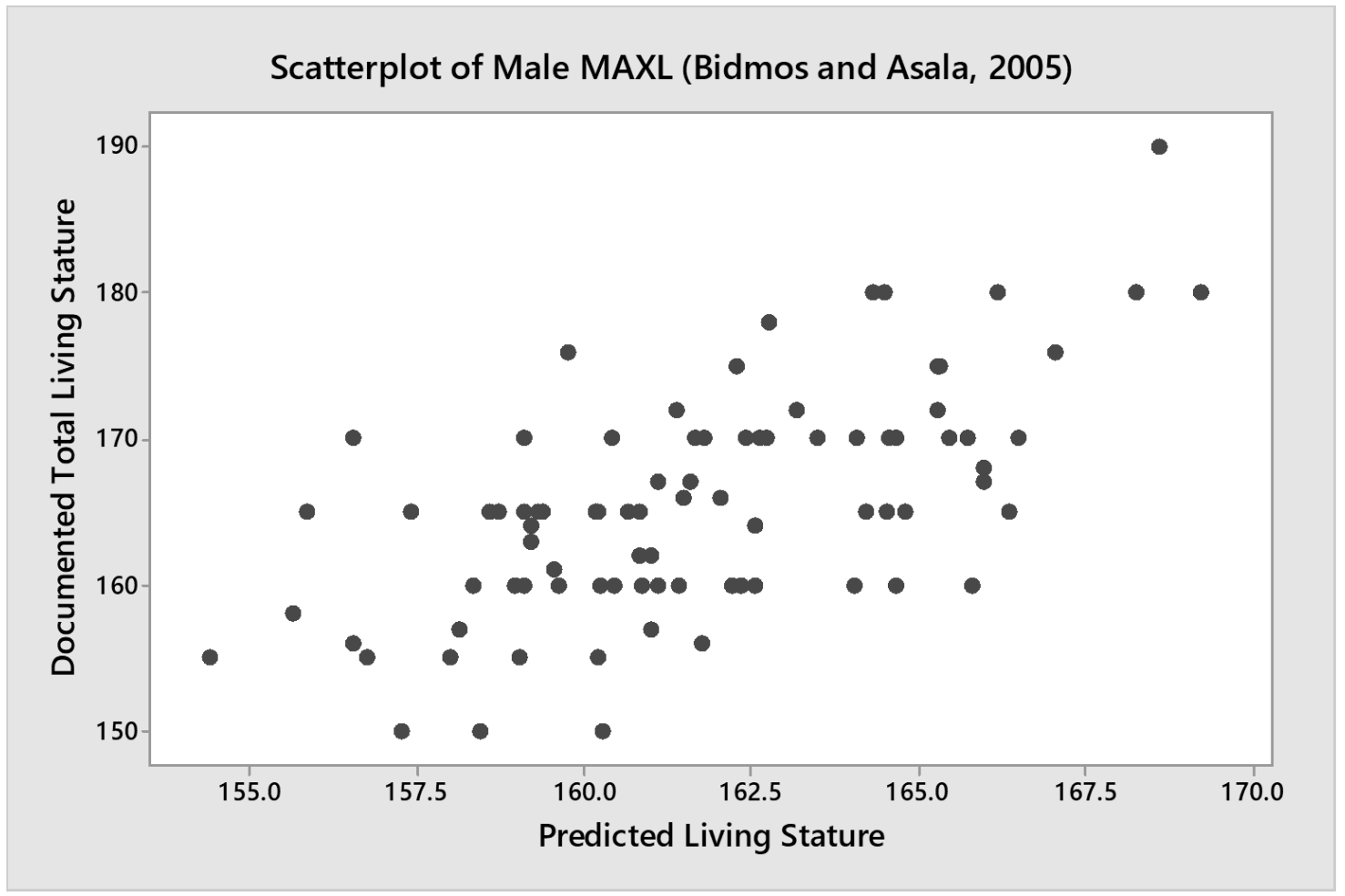

Figure 4.2 Simple scatterplot illustrating the correlation between documented and predicted living stature in the Thai male population using the male Black South African Maximum Length (MAXL) simple regression equation for the calcaneus (Bidmos and Asala, 2005).

\subsubsection{Accuracy of the Holland (1995) Talar Regression Equations}

The Thai data were also entered into the Black American (Holland, 1995) talar simple regression equations for the estimation of living stature (Table 4.8). The accuracy of each simple regression equation for the talus that was tested on the Thai sample data, is determined by the strength of the correlation coefficient. Holland (1995) did not provide multiple regression equations for the talus and therefore multiple regression equations for the talus could not be tested on the Thai sample data. For both Thai males and Thai females, the Holland (1995) simple regression equations for the talus show a statistically significant relationship between the documented living stature and predicted living stature. A Bonferroni correction of $a=0.005$ is used in this research and the $p$-values for 
both male and female talar regression equations are less than $0.005(p<0.005)$. The correlation coefficient however, indicates that this relationship is not strong $(r=0.56$ males, $r=0.55$ females). Simple scatterplots were created to analyze the correlation between talar measurement variables for the talar simple regression equations tested on the Thai sample data. A scatterplot is a figure that assists in the visualization of a relationship between two variables in two-dimensional space. A strong relationship between variables is depicted in a scatterplot as a tight cluster in which data points are close together and show a clear linear relationship. The correlation coefficient is a numerical indication of the strength of this relationship of linearity. Therefore, while both male and female simple regression equations for the talus, developed from the Black American population (Holland, 1995), show a statistically significant relationship with the documented living statures of the Thai individuals, these relationships are not strong and are not accurate predictors of stature in a Thai population. Figure 4.3 is an example of a simple scatterplot when the male Black American (Holland, 1995) MTAL simple regression equation is applied to the Thai sample data. Appendix D shows simple scatterplots when male and female Black American (Holland, 1995) simple and multiple regression equations for the talus are applied to the Thai population. 
Table 4.8 Accuracies of the male and female Black American (Holland, 1995) regression equations for the talus when applied to the Thai population.

Simple Regression Equations** Correlation ( $r$-value) $\quad p$-value

Male

1.046(MTAL) +112.26

0.56

$0.000^{*}$

\section{Female}

1.046(MTAL $)+97.55 \quad 0.55 \quad 0.000^{*}$

$* p<0.005$

$* *(\mathrm{y} /$ predicted living stature $=)$

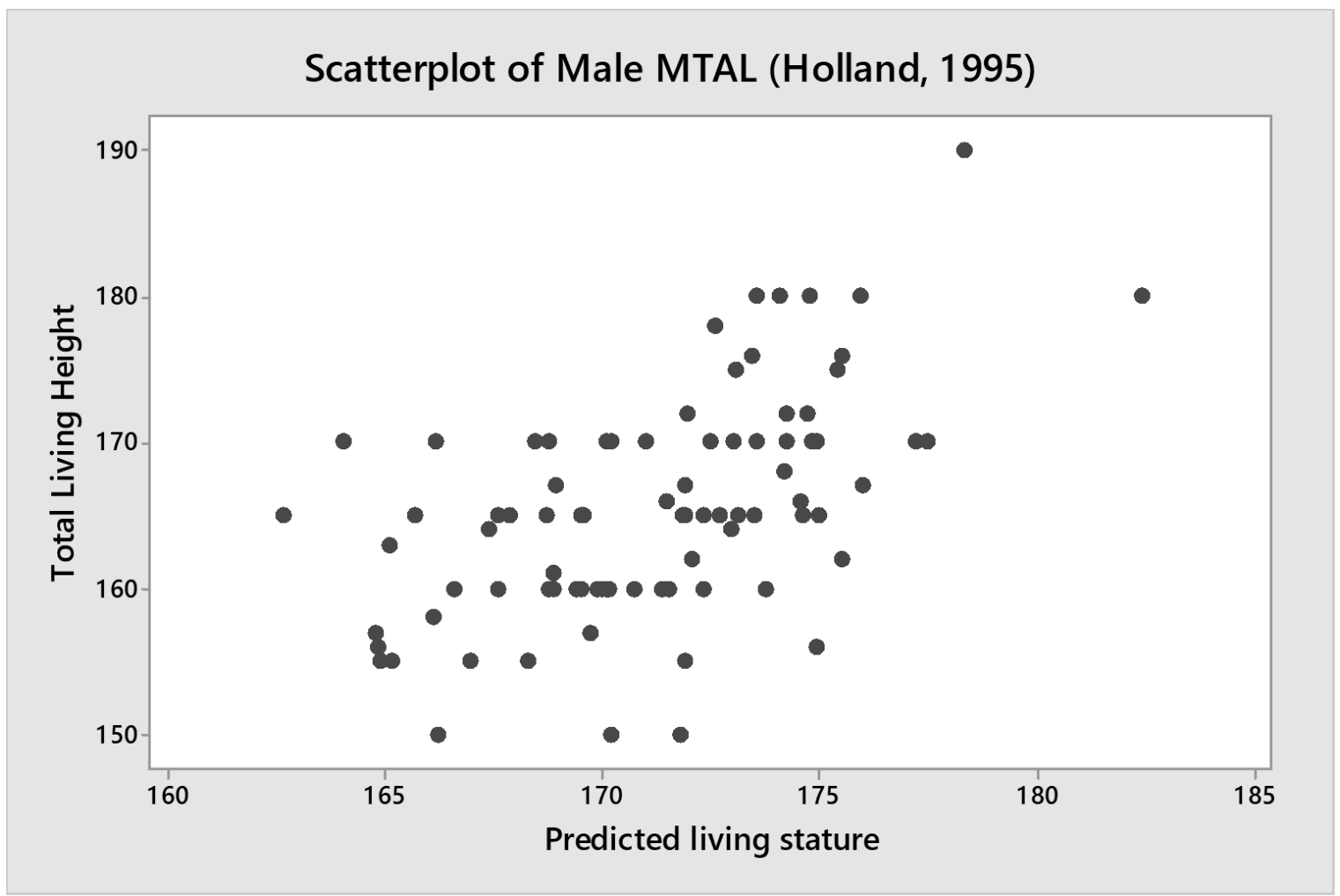

Figure 4.3 Simple scatterplot illustrating the correlation between documented and predicted living stature in the Thai male population using the male Black American (Holland, 1995) Maximum Length of the Talus (MTAL) simple regression equation. 


\subsubsection{Accuracy of Simple and Multiple Regression Equations for the Thai Population}

The calcaneus, the talus, and a combination of the two foot bones were used to create simple and multiple regression equations for living stature estimation in the Thai population. To develop the regression equations, simple and multiple regression analyses were performed using individuals from Sample 1. Unstandardized coefficients, constant values, correlation coefficients ( $r$-values), $p$-values, $f$-statistics, and the Standard Error of the Estimate (SEE) were obtained for each simple and multiple regression equation that was developed. Nine simple regression equations were calculated for each calcaneal measurement variable following Bidmos and Asala (2005). One simple regression equation was calculated for the talar measurement variable following Holland (1995).

This was done for males and females separately. A total of 18 simple regression equations were generated from the calcaneus $\left(\mathrm{N}_{\text {total calcaneal simple regression equations }}=18 ; \mathrm{N}_{\text {male calcaneal simple }}\right.$ regression equations $=9, N_{\text {female calcaneal simple regression equations }}=9$ ), and a total of two simple regression equations were generated from the talus $\left(\mathrm{N}_{\text {total talar simple regression equations }}=2 ; \mathrm{N}_{\text {male }}\right.$ talar simple regression equations $=1, \mathrm{~N}_{\text {female talar simple regression equations }}=1$ ) to estimate living stature in the Thai population. Simple regression equations for the calcaneus and the talus, for both sexes, are shown in Table 4.9. They are arranged in decreasing order of the value (strength) of the correlation coefficient ( $r$-value). and increasing magnitude of Standard Error of the Estimate (SEE). The accuracy of the estimation of stature (i.e. how close the estimate is to the documented living stature) for each simple regression equation is measured by the Standard Error of the Estimate (SEE); the lower the SEE as presented by a regression equation, the more accurate the estimate of stature. 
Table 4.9 Calcaneal and talar simple regression equations for male and female living stature estimation (in $\mathrm{cm}$ ) in a Thai population.

\begin{tabular}{lcccc}
\hline Regression Equations** & $\begin{array}{c}\text { Correlation } \\
(r \text {-value })\end{array}$ & $p$-value & $f$-statistics & SEE \\
\hline Calcaneus & & & & \\
\hline Male & & & & \\
$1.02(\mathrm{MAXL})+83.59$ & 0.63 & 0.000 & 55.44 & 5.95 \\
$1.35(\mathrm{LAL})+99.56$ & 0.50 & 0.000 & 28.50 & 6.99 \\
$1.26(\mathrm{BH})+116.32$ & 0.48 & 0.000 & 25.16 & 6.73 \\
$1.42(\mathrm{MIDB})+107.33$ & 0.46 & 0.000 & 17.12 & 6.64 \\
$0.97(\mathrm{MAXH})+123.18$ & 0.45 & 0.000 & 21.61 & 6.84 \\
$1.87(\mathrm{MINB})+117.43$ & 0.41 & 0.000 & 22.66 & 6.81 \\
$1.38(\mathrm{CFH})+128.53$ & 0.40 & 0.000 & 15.65 & 7.04 \\
$1.12(\mathrm{DAFB})+132.55$ & 0.28 & $0.010 *$ & 06.99 & 7.37 \\
$1.09(\mathrm{DAFL})+133.53$ & 0.28 & $0.009 *$ & 07.09 & 7.36 \\
& & & & \\
Female & & & & \\
$0.90(\mathrm{MAXL})+86.94$ & 0.54 & 0.000 & 34.54 & 3.06 \\
$1.53(\mathrm{LAL})+85.77$ & 0.53 & 0.000 & 33.26 & 6.09 \\
$0.94(\mathrm{MAXH})+115.94$ & 0.47 & 0.000 & 24.18 & 6.34 \\
$1.19(\mathrm{BH})+112.27$ & 0.41 & 0.000 & 16.98 & 6.56 \\
$1.62(\mathrm{DAFB})+111.07$ & 0.40 & 0.000 & 16.03 & 6.59 \\
$1.55(\mathrm{DAFL})+113.08$ & 0.39 & 0.000 & 14.89 & 6.63 \\
$1.16(\mathrm{MIDB})+110.13$ & 0.35 & 0.001 & 11.91 & 6.73 \\
$0.97(\mathrm{CFH})+129.58$ & $0.021^{*}$ & 05.49 & 6.96 \\
$0.86(\mathrm{MINB})+133$ & $0.034^{*}$ & 04.65 & 6.99
\end{tabular}

\begin{tabular}{lllll}
\hline Talus & & & & \\
\hline Male & & & & \\
$1.22(\mathrm{MTAL})+96.73$ & 0.56 & 0.000 & 38.91 & 6.34
\end{tabular}

Female

$\begin{array}{lllll}1.25(\mathrm{MTAL})+90.62 & 0.55 & 0.000 & 36.48 & 6.01\end{array}$

$* p>0.005$

$* *(\mathrm{y} /$ predicted living stature $=)$ 
Using both direct and stepwise statistical methods, multiple regression equations were developed for males and females separately using only calcaneal measurement variables, as well as by using a combination of calcaneal and talar measurement variables. Using the direct statistical method, a multiple regression equation was generated using all nine calcaneal measurement variables to determine whether accuracy rates are enhanced when utilizing all available calcaneal measurement variables. Using a stepwise statistical method, calcaneal multiple regression equations were generated for males and females in order to determine which combination of calcaneal measurement variables produced the most accurate estimation of living stature. Multiple regression function 5 (Table 4.10) was developed using a stepwise method for both males and females. The MTAL talar measurement variable was added to each male and female calcaneal multiple regression equation that was developed, thus creating additional multiple regression equations. In total, 10 multiple regression equations were generated for living stature estimation using calcaneal and talar measurement variables $\left(\mathrm{N}_{\text {total multiple regression equations }}=10 ; \mathrm{N}_{\text {male multiple }}\right.$ regression equations $=5, \mathrm{~N}_{\text {female multiple regression equations }}=5$ ). Refer to Table 4.10 for the multiple regression equations generated for both sexes. As with the simple regression equations generated in this study, the accuracy of the estimation of stature (i.e. how close the estimate is to the documented living stature) for each multiple regression equation is measured by the Standard Error of the Estimate (SEE); the lower the SEE as presented by a regression equation, the more accurate the estimate of stature. 
Table 4.10 Direct and stepwise multiple regression equations for male and female living stature estimation (in $\mathrm{cm}$ ).

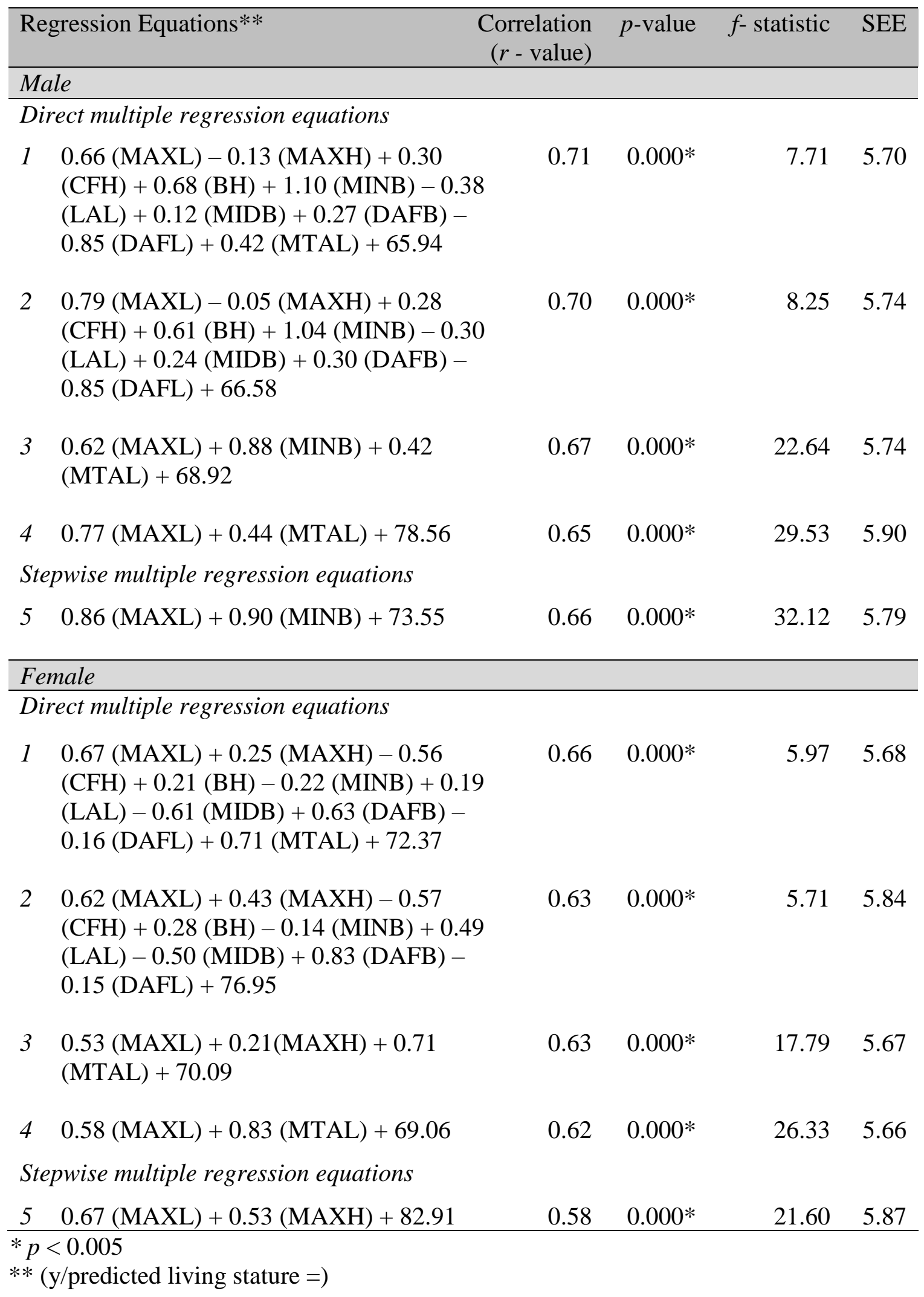


The unstandardized coefficients and constant values are used in developing both calcaneal and talar simple and multiple regression equations for stature estimation in the Thai population. The estimate of living stature is the sum of the products of the unstandardized coefficient and the magnitude of the corresponding measurement variables (in $\mathrm{mm}$ ) and the constant value, or intercept. For example, individual 31 (ID no. 31) from the Thai sample has a maximum calcaneal length (MAXL) of $70.81 \mathrm{~mm}$. Using the associated calcaneal simple regression equation (Table 4.9), this individual would have a predicted living stature of:

$$
\begin{aligned}
& \text { Predicted living stature }=1.02(\mathrm{MAXL})+83.58 \\
& \text { Predicted living stature }=1.02(70.81)+83.58 \\
& \text { Predicted living stature }=155.81 \mathrm{~cm}
\end{aligned}
$$

Following the protocol of Bidmos and Asala (2005), the current author obtained a predicted range for living stature. To do this, the Standard Error of the Estimate (SEE) is either added to, or subtracted from, the final estimate of living stature as predicted by the calcaneal simple regression equation. In this case, the Standard Error of the Estimate (SEE) was $5.93 \mathrm{~cm}$. Therefore, the value of $1 \mathrm{SEE}$ is $5.93 \mathrm{~cm}$ and the value of $2 \mathrm{SEEs}$ is $11.86 \mathrm{~cm}$. As a $2 \mathrm{SEE}$ interval provides a more accurate estimate of total living stature, the current author recommends that a 2SEE interval is utilized for all predicted living stature ranges (Giles and Klepinger, 1988). Therefore, the range of predicted living stature at 2SEE is:

Predicted living stature range $=155.81 \mathrm{~cm} \pm 11.86 \mathrm{~cm}$ Predicted living stature range $=143.95 \mathrm{~cm}-167.67 \mathrm{~cm}$

This means that the predicted living stature for the calcaneus of individual (ID no. 31) ranged from $143.95 \mathrm{~cm}$ to $167.67 \mathrm{~cm}$. All calcaneal and talar simple and multiple 
regression equations developed (Table 4.9 and Table 4.10) can be used this way for future practical applications.

Nine simple regression equations were calculated for each calcaneal measurement variable following Bidmos and Asala (2005). This was done for males and females separately. A total of 18 simple regression equations were generated to estimate living stature from the calcaneus for the Thai population $\left(\mathrm{N}_{\text {total calcaneal simple regression equations }}=18\right.$; $\left.\mathrm{N}_{\text {male calcaneal simple regression equations }}=9, \mathrm{~N}_{\text {female calcaneal simple regression equations }}=9\right)($ Table 4.9). Pvalues were obtained to determine whether a statistically significant relationship existed between documented living stature and the living stature of an individual as predicted by the corresponding calcaneal regression equation. A statistical significance level of 5\% error rate $(a=0.05)$ is used in this research and was adjusted by dividing the Bonferroni correction by the number of testable measurement variables $(a=0.05 / 10)$ to give a Bonferroni correction of $a=0.005$. Therefore, if the $p$-value is less than, or equal to, 0.005 $(\mathrm{p} \leq 0.005)$, a statistically significant relationship exists between documented living stature and predicted living stature for the Thai individual.

For Thai males, all calcaneal measurement variables showed a statistically significant positive correlation with documented living stature except the Dorsal Articular Facet Breadth (DAFB) and the Dorsal Articular Facet Length (DAFL) calcaneal measurement variables. In other words, DAFB and DAFL did not show statistically significant correlations with documented living stature when individual calcaneal measurement variables were analyzed using a Bonferroni correction of $a=0.005$. For the male calcaneal simple regression equations, the correlation coefficients ( $r$-values) ranged from 0.28 to 0.63 and the Standard Error of the Estimate (SEE) ranged from 5.95 to 7.36 (Table 4.9). The male Maximum Length (MAXL) calcaneal measurement variable 
showed the highest correlation with documented living stature $(r=0.63)$ and the lowest SEE (5.95) (Table 4.9), which indicates that this measurement variable is the most accurate predictor of stature for Thai males.

For Thai females, seven of the nine calcaneal measurement variables showed a significant positive correlation with documented living stature; the Cuboidal Facet Height (CFH) and Minimum Breadth (MINB) calcaneal measurement variables did not display a significant positive correlation with documented living stature. Overall, for the female calcaneal simple regression equations, the correlation coefficients ( $r$-values) range from 0.23 to 0.54 . The Standard Error of the Estimate (SEE) values range from 3.06 to 6.99, which is lower than the Standard Error of the Estimate (SEE) values observed in the male calcaneal simple regression equations. For the calcaneus, this indicates that the female simple regression equations provide a more accurate estimate of living stature than the male equations. Similar to males, the highest correlation with documented living stature was shown by the MAXL calcaneal measurement variable $(r=0.54)$. The Standard Error of the Estimate (SEE) for the female MAXL calcaneal simple regression equation however, is notably lower than that presented in the male MAXL calcaneal simple regression equation $(\mathrm{SEE}=3.06$ in females, $\mathrm{SEE}=5.95$ in males). This indicates that the female MAXL simple regression equation provides a more accurate estimate of living stature than the male MAXL simple regression equation.

Following Holland (1995), two simple regression equations were calculated using the Maximum Length of the Talus (MTAL) talar measurement variable. This was done separately for both males and females, which resulted in the generation of two talar simple regression equations for the estimation of living stature for the Thai population $\left(\mathrm{N}_{\text {total talar simple regression equations }}=2 ; \mathrm{N}_{\text {male talar simple regression equations }}=1, \mathrm{~N}_{\text {female talar simple regression }}\right.$ 
equations=1) (Table 4.9). As with the simple regression equations generated from the calcaneus, $p$-values were obtained and a Bonferonni correction of $a=0.005$ was utilized $(p=0.005)$. The MTAL talar measurement variable showed a significant positive correlation with documented living stature in both Thai males and Thai females. The male $(r=0.56)$ and female $(r=0.55)$ correlation coefficient values were similar. The Standard Error of the Estimate (SEE) was lower in Thai females $(\mathrm{SEE}=6.01)$ than in Thai males $(\mathrm{SEE}=6.34)$. This indicates that although males show a slightly higher correlation coefficient, the female talar simple regression equations may provide a more accurate estimate of living stature.

Multiple regression equations were also developed for living stature estimation in a Thai population. Using both direct and stepwise statistical methods, five multiple regression equations were developed for Thai males, and five multiple regression equations were developed for Thai females using calcaneal measurement variables, as well as a combination of calcaneal and talar measurement variables $\left(\mathrm{N}_{\text {total multiple regression }}\right.$ equations $\left.=10 ; \mathrm{N}_{\text {male multiple regression equations }}=5, \mathrm{~N}_{\text {female multiple regression equations }}=5\right)($ Table 4.10). All multiple regression equations show a significant positive correlation with documented living stature $(p<0.005)$ for the Thai population. For both males and females, the correlation coefficient values ( $r$-values) from the multiple regression equations range from 0.58 to 0.71 , which is higher than those obtained from the use of individual measurement variables (i.e. simple regression equations). This indicates that the multiple regression equations are more accurate predictors for stature estimation than the simple regression equations in the Thai population. Standard Error of the Estimate (SEE) values were also obtained for each multiple regression equation. The Standard Error of the Estimate (SEE), for each multiple regression equation, ranged from 5.70 to 5.90 for Thai 
males and 5.68 to 5.87 for Thai females. Overall, the Standard Error of the Estimate (SEE) values are lower than those obtained from the use of individual measurements. This indicates that the multiple regression equations are more accurate predictors of living stature than the simple regression equations in the Thai population.

Using a direct statistical method, a multiple regression equation was generated using all nine calcaneal measurement variables for males $(r=0.70, \mathrm{SEE}=5.74)$ and females $(r=0.63, \mathrm{SEE}=5.84)($ Table 4.10). Using a stepwise statistical method, a multiple regression equation was produced for males and for females using only calcaneal measurement variables. For males, the stepwise multiple regression equation (male multiple regression equation 5) was generated using the Maximum Length (MAXL) and Minimum Breadth $(\mathrm{MINB})$ calcaneal measurement variables $(r=0.66, \mathrm{SEE}=5.79)$ (Table 4.10). For females, the stepwise multiple regression equation (female multiple regression equation 5) was generated using the Maximum Length (MAXL) and Maximum Height $(\mathrm{MAXH})$ calcaneal measurement variables $(r=0.58, \mathrm{SEE}=5.87)$ (Table 4.10).

To determine whether the talus added to the utility of the regression equations (i.e. if using both the calcaneus and the talus produced more accurate regression equations for the estimation of living stature in a Thai population), comparisons were made between regression equations that utilized the MTAL talar variable and those that did not (Table 4.10). Using a direct statistical method, the MTAL talar measurement variable was added to the calcaneal simple regression equation that most accurately predicted living stature in the Thai population, for both males and females. The MAXL simple regression equation for the calcaneus demonstrated the highest correlation and lowest Standard Error of the Estimate (SEE) for both sexes and thus is the most accurate predictor of living stature for 
the Thai population (Table 4.9). When the male MTAL talar measurement variable was added to the male MAXL calcaneal simple regression equation, the correlation coefficient increased from 0.63 (Table 4.9) to 0.65 (Table 4.10), and the Standard Error of the Estimate (SEE) decreased from 5.95 (Table 4.9) to 5.90 (Table 4.10). When the female MTAL talar measurement variable was added to the female MAXL calcaneal simple regression equation, the correlation coefficient increased from 0.54 (Table 4.9) to 0.62 (Table 4.10), but the Standard Error of the Estimate (SEE) increased from 3.06 (Table 4.9) to 5.66 (Table 4.10). This indicates that calcaneal multiple regression equations, which include the MTAL talar measurement variable, may not be as accurate for the estimation of stature in a Thai population as the simple regression equations that utilize only the MAXL measurement of the calcaneus.

Using a direct statistical method, the MTAL talar measurement variable was also added to the multiple regression equations generated solely from calcaneal measurement variables to determine if the talus added to the utility of the multiple regression equations for a Thai population (Table 4.10). When the male MTAL talar measurement variable was added to the male multiple regression equation, which utilized all nine calcaneal variables, the correlation coefficient increased from 0.70 (male multiple regression equation 2) to 0.71 (male multiple regression equation 1) in the Thai population, and the Standard Error of the Estimate (SEE) decreased from 5.74 (male multiple regression equation 2) to 5.70 (male multiple regression equation 1). When the male MTAL talar measurement variable was added to the male multiple regression equation, which utilized the MAXL and MINB calcaneal measurement variables, the correlation coefficient ( $r$ value) increased from 0.66 (male multiple regression equation 5) to 0.67 (male multiple regression equation 3) in the Thai population, and the Standard Error of the Estimate 
(SEE) decreased from 5.79 (male multiple regression equation 5) to 5.74 (male multiple regression equation 3). When the female MTAL talar measurement variable was added to the female multiple regression equation, which utilized all nine calcaneal variables, the correlation coefficient values ( $r$-values) increased 0.63 (female multiple regression equation 2) to 0.66 (female multiple regression equation 1) in the Thai population, and the Standard Error of the Estimate (SEE) decreased from 5.84 (female multiple regression equation 2) to 5.68 (female multiple regression equation 1). When the female MTAL talar measurement variable was added to the female multiple regression equation, which utilized the MAXL and MAXH calcaneal measurement variables, the correlation coefficient ( $r$-value) increased to from 0.58 (female multiple regression equation 5) to 0.63 (female multiple regression equation 3) in the Thai population, and the Standard Error of the Estimate (SEE) decreased from 5.87 (female multiple regression equation 5) to 5.67 (female multiple regression equation 3).

Overall, for both males and females, regression equation correlation coefficients increased and Standard Error of the Estimate (SEE) values decreased when the MTAL talar measurement variable was added to the multiple regression equations (Table 4.10). The highest correlation coefficient ( $r$-value) and the lowest Standard Error of the Estimate (SEE) value for males was shown in multiple regression equation 1 that uses all calcaneal and talar measurement variables to predict living stature $(r=0.71$ for males, SEE $=5.70)$. This indicates a stronger relationship between documented living stature and predicted living stature for males in the Thai collection when the talus is utilized. It also shows that the inclusion of the talus provides a more accurate estimation of living stature. While multiple regression equation 1 (using all calcaneal and talar measurements) for females also shows the highest correlation coefficient $(r=0.68)$, this regression equation does not 
demonstrate the lowest Standard Error of the Estimate (SEE) value. The female multiple regression equation with the lowest Standard Error of the Estimate (SEE) (i.e. the female multiple regression equation which is the most accurate) is female multiple regression equation 4, which uses the MAXL and MTAL measurement variables (SEE = 5.66). However, this equation also utilizes the talar measurement variable, which indicates that, overall, the inclusion of the talus is beneficial for stature estimation.

\subsection{Cross-Validation of the Thai Regression Equations}

Sample 1 was used to derive calcaneal and talar simple and multiple regression equations for the Thai population. Sample 2 is an independent sample, which was used to test the accuracy of the calcaneal and talar simple and multiple regression equations from Sample 1. Only simple and multiple regression equations of the calcaneus and talus that showed a significant positive correlation with documented living stature $(p<0.005)$ in the Thai population were tested on Sample 2. The predicted living stature ranges, obtained from the calcaneal and talar simple and multiple regression equations from Sample 1, were correlated with documented living stature in the Thai population. P-values were calculated from the calcaneal and talar simple and multiple regression equations generated from Sample 1 to determine if there was a statistically significant relationship between documented living stature and the predicted living stature of a Thai individual. Correlation coefficients ( $r$-values) were also calculated. Following the protocol of Bidmos and Asala (2005) for the calcaneus and Holland (1995) for the talus, the accuracy of the estimation of living stature for each of the calcaneal and talar simple and multiple regression equations was assessed. This was done by calculating in what percent of cases, 
from Sample 2, did the Thai predicted living stature fall within 1 Standard Error of the Estimate (1SEE) and 2 Standard Errors of the Estimate (2SEE) of the Thai documented living stature. Table 4.11 shows the male and female simple regression equations derived from the calcaneus and the talus (tested on Sample 2) and the associated accuracy rates. The male and female multiple regression equations that were tested on Sample 2 and their associated accuracy rates are available in Table 4.12. 
Table 4.11 Cross validation results of calcaneal and talar simple regression equations for the Thai population.

\begin{tabular}{lcccc}
\hline Regression Equations & $\begin{array}{c}\text { Correlation } \\
(r \text {-value })\end{array}$ & $p$-value & 1SEE & 2SEE \\
\hline Calcaneus & & & & \\
\hline Male & & & & \\
& & & & \\
$1.35(\mathrm{LAL})+99.56$ & 0.56 & $0.001 *$ & $67 \%$ & $97 \%$ \\
$1.02(\mathrm{MAXL})+83.59$ & 0.55 & $0.002^{*}$ & $50 \%$ & $90 \%$ \\
$1.26(\mathrm{BH})+116.32$ & 0.52 & $0.003 *$ & $53 \%$ & $90 \%$ \\
$0.97(\mathrm{MAXH})+123.18$ & 0.34 & 0.063 & $53 \%$ & $93 \%$ \\
$1.87(\mathrm{MINB})+117.43$ & 0.29 & 0.122 & $53 \%$ & $90 \%$ \\
$1.42(\mathrm{MIDB})+107.33$ & 0.23 & 0.216 & $53 \%$ & $90 \%$ \\
$1.38(\mathrm{CFH})+128.53$ & 0.08 & 0.672 & $57 \%$ & $87 \%$
\end{tabular}

Female

$\begin{array}{lrrrr}1.55(\mathrm{DAFL})+113.08 & 0.52 & 0.003 * & 67 \% & 97 \% \\ 0.90(\mathrm{MAXL})+86.94 & 0.50 & 0.004^{*} & 33 \% & 67 \% \\ 1.19(\mathrm{BH})+112.27 & 0.38 & 0.036 & 70 \% & 93 \% \\ 1.53(\mathrm{LAL})+85.77 & 0.35 & 0.061 & 63 \% & 93 \% \\ 0.94(\mathrm{MAXH})+115.94 & 0.35 & 0.061 & 53 \% & 90 \% \\ 1.16(\mathrm{MIDB})+110.13 & 0.30 & 0.102 & 63 \% & 97 \% \\ 1.62(\mathrm{DAFB})+111.07 & 0.09 & 0.624 & 63 \% & 93 \%\end{array}$

\begin{tabular}{llccc}
\hline Talus & & & & \\
\hline Male & & & & \\
$1.22(\mathrm{MTAL})+96.73$ & 0.44 & 0.016 & $53 \%$ & $90 \%$ \\
Female & & & & \\
$1.25(\mathrm{MTAL})+90.62$ & 0.42 & 0.021 & $70 \%$ & $93 \%$
\end{tabular}

$* p<0.005$

$* *(\mathrm{y} /$ predicted living stature $=)$ 
Table 4.12 Cross validation results of calcaneal and talar multiple regression equations for the Thai population.

\begin{tabular}{|c|c|c|c|c|c|}
\hline \multicolumn{2}{|c|}{ Regression Equations** } & $\begin{array}{c}\text { Correlation } \\
(r \text {-value })\end{array}$ & $p$-value & 1SEE & 2SEE \\
\hline \multicolumn{6}{|c|}{ Male } \\
\hline \multicolumn{6}{|c|}{ Direct multiple regression equations } \\
\hline 1 & $\begin{array}{l}0.66(\mathrm{MAXL})-0.13(\mathrm{MAXH})+0.30 \\
(\mathrm{CFH})+0.68(\mathrm{BH})+1.10(\mathrm{MINB})-0.38 \\
(\mathrm{LAL})+0.12(\mathrm{MIDB})+0.27(\mathrm{DAFB})- \\
0.85(\mathrm{DAFL})+0.42(\mathrm{MTAL})+65.94\end{array}$ & 0.45 & 0.013 & $47 \%$ & $83 \%$ \\
\hline 2 & $\begin{array}{l}0.79(\mathrm{MAXL})-0.05(\mathrm{MAXH})+0.28 \\
(\mathrm{CFH})+0.61(\mathrm{BH})+1.04(\mathrm{MINB})-0.30 \\
(\mathrm{LAL})+0.24(\mathrm{MIDB})+0.30(\mathrm{DAFB})- \\
0.85(\mathrm{DAFL})+66.58\end{array}$ & 0.44 & 0.015 & $57 \%$ & $83 \%$ \\
\hline 3 & $\begin{array}{l}0.62(\mathrm{MAXL})+0.88(\mathrm{MINB})+0.42 \\
(\mathrm{MTAL})+68.92\end{array}$ & 0.59 & $0.001 *$ & $53 \%$ & $87 \%$ \\
\hline 4 & $0.77(\mathrm{MAXL})+0.44(\mathrm{MTAL})+78.56$ & 0.57 & $0.001 *$ & $57 \%$ & $87 \%$ \\
\hline & pwise multiple regression equations & & & & \\
\hline 5 & $0.86(\mathrm{MAXL})+0.90(\mathrm{MINB})+73.55$ & 0.57 & $0.001 *$ & $53 \%$ & $90 \%$ \\
\hline \multicolumn{6}{|c|}{ Female } \\
\hline \multicolumn{6}{|c|}{ Direct multiple regression equations } \\
\hline 1 & $\begin{array}{l}0.67(\mathrm{MAXL})+0.25(\mathrm{MAXH})-0.56 \\
(\mathrm{CFH})+0.21(\mathrm{BH})-0.22(\mathrm{MINB})+0.19 \\
(\mathrm{LAL})-0.61(\mathrm{MIDB})+0.63(\mathrm{DAFB})- \\
0.16(\mathrm{DAFL})+0.71(\mathrm{MTAL})+72.37\end{array}$ & 0.46 & 0.010 & $67 \%$ & $87 \%$ \\
\hline 2 & $\begin{array}{l}0.62(\mathrm{MAXL})+0.43(\mathrm{MAXH})-0.57 \\
(\mathrm{CFH})+0.28(\mathrm{BH})-0.14(\mathrm{MINB})+0.49 \\
(\mathrm{LAL})-0.50(\mathrm{MIDB})+0.83(\mathrm{DAFB})- \\
0.15(\mathrm{DAFL})+76.95\end{array}$ & 0.41 & 0.024 & $63 \%$ & $90 \%$ \\
\hline 3 & $\begin{array}{l}0.53(\mathrm{MAXL})+0.21(\mathrm{MAXH})+0.71 \\
(\mathrm{MTAL})+70.09\end{array}$ & 0.48 & 0.007 & $70 \%$ & $93 \%$ \\
\hline 4 & $\begin{array}{l}0.58(\mathrm{MAXL})+0.83(\mathrm{MTAL})+69.06 \\
\text { pwise multiple regression equations }\end{array}$ & 0.50 & $0.005^{*}$ & $63 \%$ & $93 \%$ \\
\hline 5 & $0.67(\mathrm{MAXL})+0.53(\mathrm{MAXH})+82.91$ & 0.48 & 0.015 & $67 \%$ & $93 \%$ \\
\hline
\end{tabular}


Simple scatterplots were created for each calcaneal and talar simple and multiple regression equation. A scatterplot is a figure that assists in the visualization of a relationship between two variables in two-dimensional space. These scatterplots illustrate the strength of the relationship between documented living stature and the predicted living stature in the Thai population. A strong relationship between variables is illustrated in a scatterplot as a tight cluster in which data points are close together and show a clear linear relationship. Figure 4.4 is an example of a simple scatterplot, illustrating the crossvalidation correlation for the Thai male Maximum Length (MAXL) calcaneal simple regression equation. Refer to Appendix E for the simple scatterplots for each Thai calcaneal and talar simple and multiple regression equation tested on Sample 2.

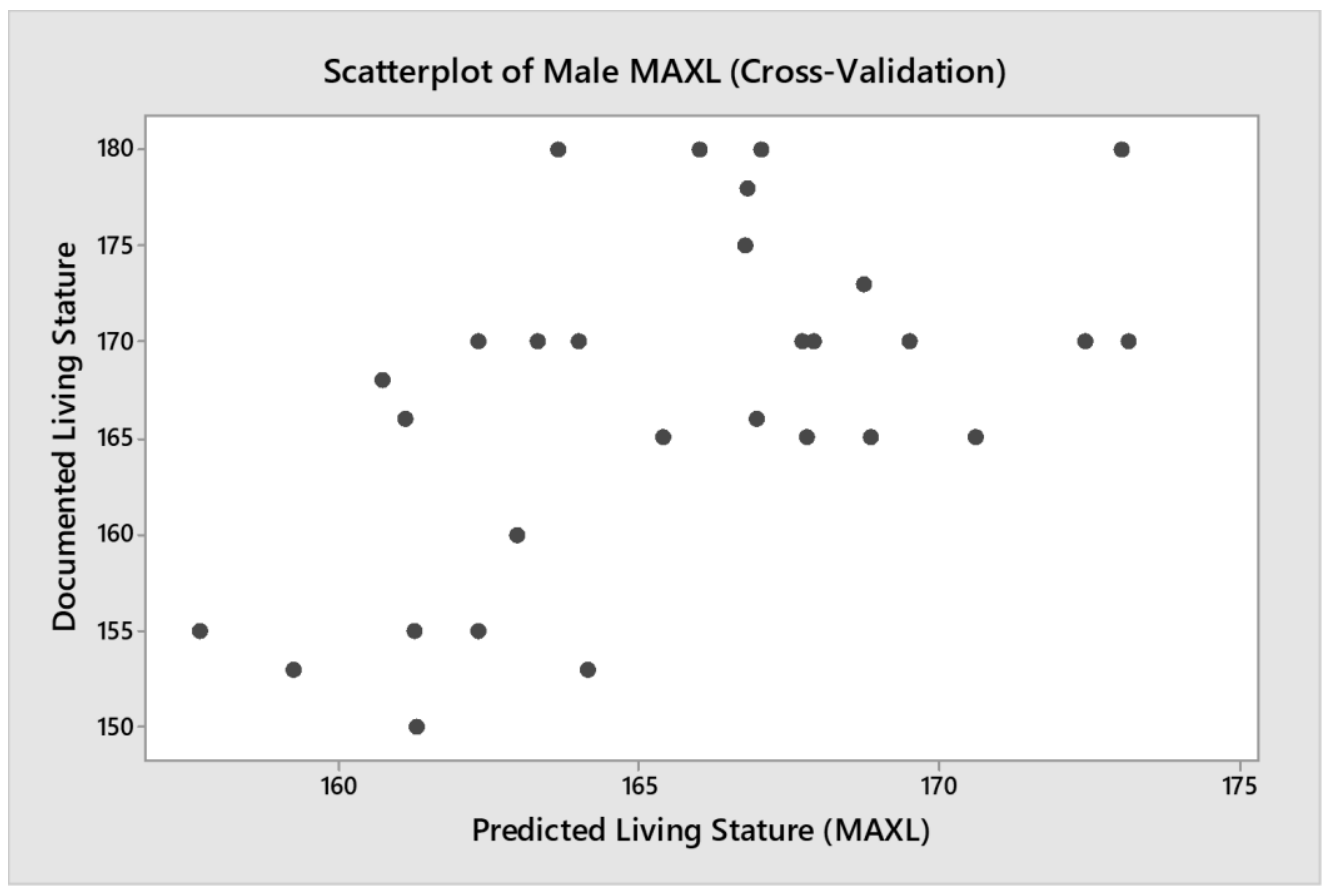

Figure 4.4 Simple scatterplot illustrating the correlation between documented and predicted living stature, in a Thai population, using the Maximum Length (MAXL) calcaneal simple regression equation. 
For the calcaneus, fourteen of the simple regression equations generated from Sample 1 were tested on Sample $2\left(\mathrm{~N}_{\text {total calcaneal simple regression equations }}=14 ; \mathrm{N}_{\text {male calcaneal simple }}\right.$ regression equations $=7, \mathrm{~N}_{\text {female calcaneal simple regression equations }}=7$ ). This was done for males and females separately. Only simple regression equations for the calcaneus that showed a significant positive correlation with documented living stature $(p<0.005)$ were tested on Sample 2. P-values were obtained to determine whether a statistically significant relationship existed between documented living stature and predicted living stature of a Thai individual. A statistical significance level of $5 \%$ error rate $(a=0.05)$ was used in this research and was adjusted by dividing the Bonferroni correction by the number of testable measurement variables $(a=0.05 / 10)$ to give a Bonferroni correction of $a=0.005$. Therefore, if the $\mathrm{p}$-value is less than, or equal to, $0.005(p \leq 0.005)$, a statistically significant relationship exists between documented living stature and predicted living stature.

For Thai males, only the Load Arm Length (LAL), Maximum Length (MAXL), and Body Height $(\mathrm{BH})$ calcaneal measurement variables showed a significant positive correlation with documented living stature. The highest correlation was shown by the LAL calcaneal measurement variable (Table 4.11). For Thai females, only the Dorsal Articular Facet Length (DAFL) and Maximum Length (MAXL) calcaneal measurement variables showed a significant positive correlation with documented living stature. The highest correlation was shown by the DAFL calcaneal measurement variable (Table 4.11). Table 4.11 also shows the results of the accuracy of stature estimation using calcaneal simple regression equations at 1 and 2 Standard Errors of the Estimate (SEE). In males, the estimated living stature fell within 1 SEE in $50 \%$ to $67 \%$ of cases while the 
accuracy was higher at $2 \operatorname{SEE}(87 \%$ to $97 \%)$. For Thai females, living stature estimates fell within 1SEE in $53 \%$ to $70 \%$ of cases and within 2 SEE in $90 \%$ to $97 \%$ of cases.

Two talar simple regression equations that were generated from Sample 1 were tested on Sample 2. This was done separately for both males and females $\left(\mathrm{N}_{\text {total talar simple }}\right.$ regression equations $=2 ; \mathrm{N}_{\text {male talar simple regression equations }}=1, \mathrm{~N}_{\text {female talar simple regression equations }}=1$ ) (Table 4.11). As with the calcaneal simple regression equations, p-values were obtained and a Bonferonni correction of $a=0.005$ was utilized. Neither the male or female talar simple regression equations demonstrated a significant positive correlation with documented living stature when tested on Sample 2. The accuracy of the living stature estimation for each of the talar simple regression equations was assessed by calculating in what percentage of cases did predicted living stature fall within 1 Standard Error of the Estimate (1SEE) and 2 Standard Errors of the Estimate (2SEE) of documented living stature in the Thai population. In males, the living stature estimate generated from the MTAL simple regression equation for the talus fell within 1SEE in 53\% of cases and within 2 SEE in $90 \%$ of cases. For Thai females, the living stature estimations generated from the MTAL simple regression equation for the talus fell within $1 \mathrm{SEE}$ in $70 \%$ of cases and within 2 SEE in $93 \%$ of cases.

Ten multiple regression equations were generated from Sample 1 using calcaneal measurement variables as well as a combination of calcaneal and talar measurement variables $\left(\mathrm{N}_{\text {total multiple regression equations }}=10 ; \mathrm{N}_{\text {male multiple regression equations }}=5, \mathrm{~N}_{\text {female multiple }}\right.$ regression equations $=5$ ). These equations were tested on Sample 2 (Table 4.12). In order to determine whether a statistically significant relationship existed between documented living stature and the predicted living stature of a Thai individual, p-values were obtained. A statistical significance level of $5 \%$ error rate $(a=0.05)$ is used in this research and was 
adjusted by dividing the Bonferroni correction by the number of testable variables $(\mathrm{a}=$ $0.05 / 10$ ) to give a Bonferroni correction of $a=0.005$. Therefore, if the $p$-value is less than, or equal to, $0.005(p \leq 0.005)$, a statistically significant relationship exists between documented living stature and predicted living stature.

For Thai males, multiple regression equations 3,4 , and 5 show a statistically significant positive correlation between living stature and predicted stature in the Thai population $(p=0.001)$. Multiple regression equations 1 and 2 do not show a statistically significant positive correlation between living stature and predicted stature in the Thai population. The highest correlation between documented living stature and predicted living stature is shown by the multiple regression equation 3 which uses the Maximum Length (MAXL), Minimum Breadth (MINB), and Maximum Length of the Talus (MTAL) calcaneal measurement variables (Table 4.12).

For Thai females, only multiple regression equation 4 shows a significant positive correlation between documented living stature and predicted living stature $(p=0.005)$. For females, the highest correlation between documented living stature and predicted living stature is also shown by multiple regression equation 4 . Multiple regression equation 4 was generated using the Maximum Length (MAXL) calcaneal measurement variable and the Maximum Length of the Talus (MTAL) talar measurement variable (Table 4.12).

The accuracy of living stature estimation for each of the multiple regression equations was assessed by calculating in what percent of cases did predicted living stature fall within 1 Standard Error of the Estimate (1SEE) and 2 Standard Errors of the Estimate (2SEE) of the documented living stature in the Thai population. For Thai males, the predicted living stature fell within 1 SEE in $47 \%$ to $57 \%$ of cases while the accuracy was 
higher at 2SEE ( $83 \%$ to $90 \%$ ). Thai females however, showed a higher percentage accuracy as the predicted living stature fell within 1SEE in $63 \%$ to $70 \%$ of cases and within 2 SEE in $87 \%$ to $93 \%$ of cases.

\subsection{Statistical Population Comparison}

The measurements from the Thai sample were compared to Black South African (Bidmos and Asala, 2005), White South African (Bidmos, 2006), and Black American (Holland, 1995) populations. Two-sample t-tests were completed in order to determine if there was a difference between the total number of individuals $(N)$, mean measurement values, and the standard deviation for each calcaneal and talar measurement variable. This was done for both males (Table 4.13) and females (Table 4.14). 
Table 4.13 Comparison of male Thai mean measurements to a Black South African (Bidmos and Asala, 2005), White South African (Bidmos, 2006), and Black American (Holland, 1995) population.

\begin{tabular}{|c|c|c|c|c|c|c|c|c|c|c|c|c|}
\hline & \multicolumn{3}{|c|}{ Current Study } & \multicolumn{3}{|c|}{$\begin{array}{l}\text { Bidmos and Asala } \\
\text { (2005) }\end{array}$} & \multicolumn{3}{|c|}{$\begin{array}{l}\text { Bidmos } \\
(2006)\end{array}$} & \multicolumn{3}{|c|}{$\begin{array}{l}\text { Holland } \\
\text { (1995) }\end{array}$} \\
\hline \multirow{2}{*}{$\begin{array}{l}\text { Measurement } \\
\text { variables }\end{array}$} & \multicolumn{3}{|c|}{ Thai } & \multicolumn{3}{|c|}{ Black South African } & \multicolumn{3}{|c|}{ White South African } & \multicolumn{3}{|c|}{ Black American } \\
\hline & $n$ & $\begin{array}{l}\text { Mean } \\
(\mathrm{mm})\end{array}$ & $\mathrm{SD}$ & $n$ & $\begin{array}{l}\text { Mean } \\
(\mathrm{mm})\end{array}$ & $\mathrm{SD}$ & $n$ & $\begin{array}{l}\text { Mean } \\
(\mathrm{mm})\end{array}$ & $\mathrm{SD}$ & $n$ & $\begin{array}{l}\text { Mean } \\
(\mathrm{mm})\end{array}$ & $\mathrm{SD}$ \\
\hline Male & & & & & & & & & & & & \\
\hline MAXL & 86 & 80.49 & 4.72 & 59 & 79.71 & 3.99 & 41 & $84.02 *$ & 5.40 & - & - & - \\
\hline MAXH & 86 & 43.62 & 3.56 & 60 & 43.71 & 2.86 & 41 & $47.67 *$ & 4.11 & - & - & - \\
\hline $\mathrm{CFH}$ & 86 & 26.79 & 2.19 & 54 & $23.91 *$ & 1.94 & 41 & $25.46^{*}$ & 2.21 & - & - & - \\
\hline $\mathrm{BH}$ & 86 & 39.06 & 2.91 & 60 & $37.18 *$ & 2.86 & 41 & 39.15 & 2.79 & - & - & - \\
\hline MINB & 86 & 25.71 & 1.88 & 58 & 25.48 & 3.17 & 41 & 26.56 & 2.23 & - & - & - \\
\hline LAL & 86 & 48.68 & 2.82 & 60 & $46.18 *$ & 3.36 & 41 & 47.20 & 3.80 & - & - & - \\
\hline MIDB & 86 & 40.97 & 2.21 & 59 & $42.61 *$ & 2.56 & 41 & 42.10 & 2.29 & - & - & - \\
\hline DAFB & 86 & 29.37 & 1.88 & 60 & $23.11 *$ & 1.85 & 41 & $23.50^{*}$ & 1.91 & - & - & - \\
\hline DAFL & 86 & 29.25 & 1.95 & 60 & 30.12 & 1.85 & 41 & $31.40 *$ & 2.63 & - & - & - \\
\hline MTAL & 86 & 56.38 & 3.52 & - & - & - & - & - & - & 25 & $61.96^{*}$ & 3.59 \\
\hline
\end{tabular}


Table 4.14 Comparison of female Thai mean measurements to a Black South African (Bidmos and Asala, 2005), White South African (Bidmos, 2005), and Black American (Holland, 1995) population.

\begin{tabular}{|c|c|c|c|c|c|c|c|c|c|c|c|c|}
\hline & \multicolumn{3}{|c|}{ Current Study } & \multicolumn{3}{|c|}{$\begin{array}{l}\text { Bidmos and Asala } \\
\text { (2005) }\end{array}$} & \multicolumn{3}{|c|}{$\begin{array}{l}\text { Bidmos } \\
(2006)\end{array}$} & \multicolumn{3}{|c|}{$\begin{array}{l}\text { Holland } \\
\text { (1995) }\end{array}$} \\
\hline \multirow{2}{*}{$\begin{array}{l}\text { Measurement } \\
\text { variables }\end{array}$} & \multicolumn{3}{|c|}{ Thai } & \multicolumn{3}{|c|}{ Black South African } & \multicolumn{3}{|c|}{ White South African } & \multicolumn{3}{|c|}{ Black American } \\
\hline & $n$ & $\begin{array}{l}\text { Mean } \\
(\mathrm{mm})\end{array}$ & SD & $n$ & $\begin{array}{l}\text { Mean } \\
(\mathrm{mm})\end{array}$ & $\mathrm{SD}$ & $n$ & $\begin{array}{l}\text { Mean } \\
(\mathrm{mm})\end{array}$ & $\mathrm{SD}$ & $n$ & $\begin{array}{l}\text { Mean } \\
(\mathrm{mm})\end{array}$ & $\mathrm{SD}$ \\
\hline \multicolumn{13}{|l|}{ Female } \\
\hline MAXL & 87 & 73.44 & 4.26 & 56 & 73.38 & 4.61 & 44 & 75.45 & 4.08 & - & - & - \\
\hline MAXH & 87 & 39.53 & 3.57 & 55 & 40.36 & 2.94 & 44 & $42.95^{*}$ & 3.37 & - & - & - \\
\hline $\mathrm{CFH}$ & 87 & 24.29 & 1.82 & 53 & $20.73 *$ & 1.79 & 44 & $22.88 *$ & 1.96 & - & - & - \\
\hline $\mathrm{BH}$ & 87 & 34.29 & 2.45 & 56 & 33.69 & 2.83 & 44 & 35.30 & 3.01 & - & - & - \\
\hline MINB & 87 & 23.48 & 1.90 & 56 & $21.18 *$ & 2.65 & 44 & 23.28 & 2.15 & - & - & - \\
\hline LAL & 87 & 44.15 & 2.82 & 56 & $41.65 *$ & 3.36 & 44 & $41.96 *$ & 2.82 & - & - & - \\
\hline MIDB & 87 & 37.08 & 2.16 & 55 & $39.00^{*}$ & 2.62 & 44 & 37.66 & 2.29 & - & - & - \\
\hline DAFB & 87 & 26.06 & 1.76 & 53 & $20.61 *$ & 1.60 & 44 & $19.96 *$ & 1.77 & - & - & - \\
\hline DAFL & 87 & 25.80 & 1.78 & 54 & $27.42 *$ & 2.24 & 44 & $27.31 *$ & 1.52 & - & - & - \\
\hline MTAL & 87 & 50.12 & 3.13 & - & - & - & - & - & - & 25 & $53.67 *$ & 3.09 \\
\hline
\end{tabular}


The study by Bidmos and Asala (2005) utilized 116 complete skeletons of Black South Africans $\left(\mathrm{N}_{\text {individuals }}=116 ; \mathrm{N}_{\text {male }}=60, \mathrm{~N}_{\text {female }}=56\right)$ from the Raymond A. Dart Collection of Human Skeletons. The Thai data were compared to the study by Bidmos and Asala (2005) in order to create regression equations for living stature estimation from the calcaneus. The mean values (in millimeters) of each calcaneal measurement variable were compared between the Thai population and the Black South African population (Bidmos and Asala, 2005) using descriptive statistics (Table 4.15). 
Table 4.15 Comparison of male and female Thai measurements of the calcaneus to a Black South African population (Bidmos and Asala, 2005).

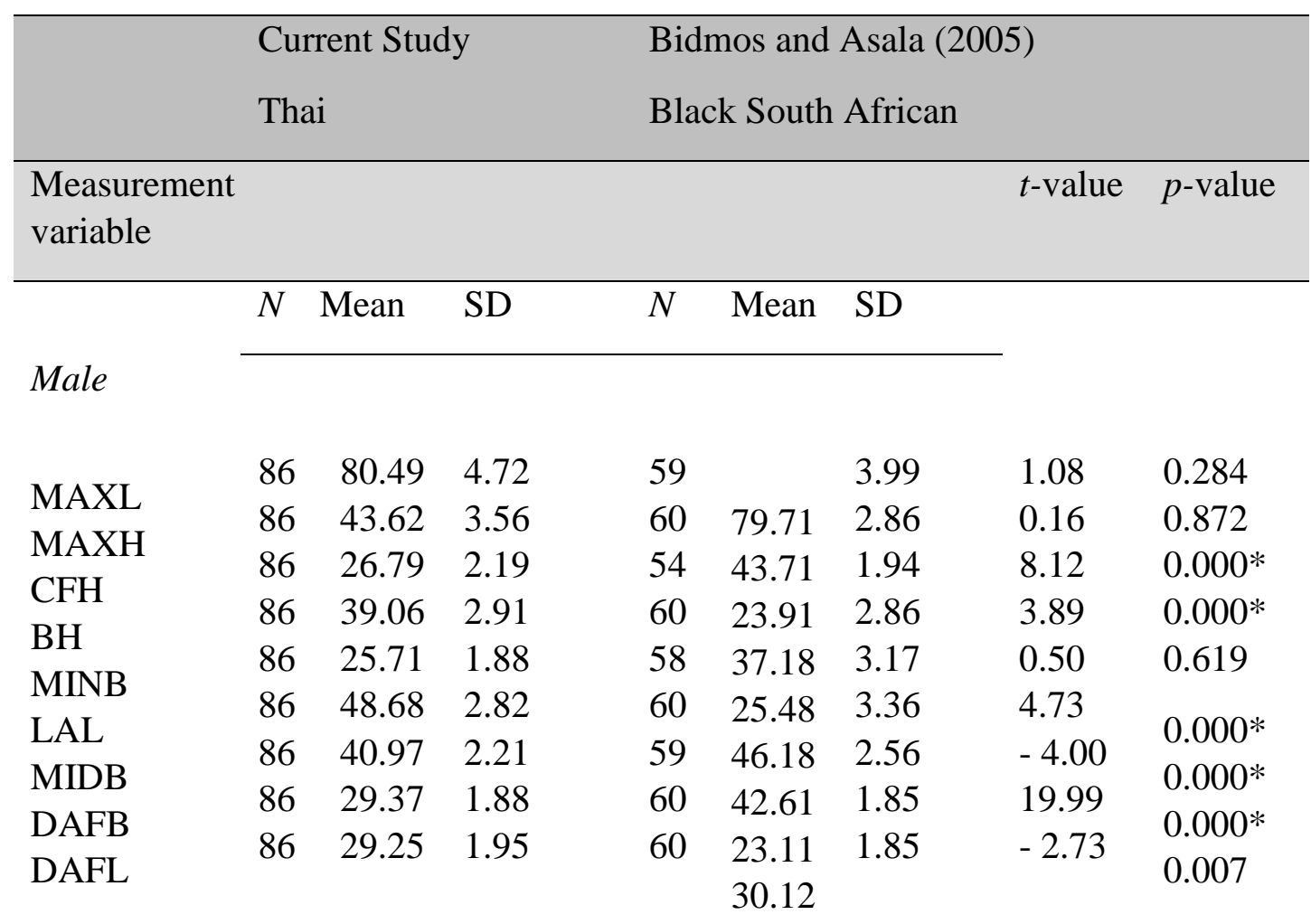

Female

$\begin{array}{lllllllll}\text { MAXL } & 87 & 73.44 & 4.26 & 56 & 73.38 & 4.61 & 0.08 & 0.939 \\ \text { MAXH } & 87 & 39.53 & 3.57 & 55 & 40.36 & 2.94 & -1.50 & 0.135 \\ \text { CFH } & 87 & 24.29 & 1.82 & 53 & 20.73 & 1.79 & 11.34 & 0.000^{*} \\ \text { BH } & 87 & 34.29 & 2.45 & 56 & 33.69 & 2.83 & 1.31 & 0.194 \\ \text { MINB } & 87 & 23.48 & 1.90 & 56 & 21.18 & 2.65 & 5.63 & 0.000^{*} \\ \text { LAL } & 87 & 44.15 & 2.82 & 56 & 41.65 & 3.36 & 4.62 & 0.000^{*} \\ \text { MIDB } & 87 & 37.08 & 2.16 & 55 & 39.00 & 2.62 & -4.55 & 0.000^{*} \\ \text { DAFB } & 87 & 26.02 & 1.76 & 53 & 20.61 & 1.60 & 18.66 & 0.000^{*} \\ \text { DAFL } & 87 & 25.80 & 1.78 & 54 & 27.42 & 2.24 & -4.52 & 0.000^{*}\end{array}$

* $p<0.005$

A statistical significance level of $5 \%$ error rate $(a=0.05)$ is used in this research and was adjusted by dividing the Bonferroni correction $(a=0.05)$ by the number of testable measurement variables to give a Bonferroni correction of $a=0.005$. A p-value 
that is less than, or equal to, $0.005(p \leq 0.005)$ indicated a statistically significant difference in the mean measurement values. A p-value greater than $0.005(p>0.005)$ indicated that there was no statistically significant difference in mean measurement variable values between populations.

For males, the means of all calcaneal measurement variables are larger in the Thai sample than in the Black South African sample (Bidmos and Asala, 2005) with the exception of Maximum Height (MAXH) and Middle Breadth (MIDB). The male Cuboidal Facet Height (CFH), Body Height (BH), Load Arm Length (LAL), Middle Breadth (MIDB), and Dorsal Articular Facet Breadth (DAFB) calcaneal measurement variables have $\mathrm{p}$-values of $0.000(p=0.000)$, which means that there is a statistically significant difference in these values between the Thai population and the Black South African population (Bidmos and Asala, 2005). In contrast, there is no statistically significant difference between the male Maximum Length (MAXL), Maximum Height (MAXH), and Minimum Breadth (MINB) calcaneal measurement variables. The p-value of the Dorsal Articular Facet Length (DAFL) calcaneal measurement variable was $\mathrm{p}=$ 0.007. While there was no "statistically significant" difference in the DAFL calcaneal measurement variable between Thai males and Black South African males (Bidmos and Asala, 2005), this measurement variable shows a higher degree of difference between these populations than the male MAXL, MAXH and MINB calcaneal measurement variables.

For females, the mean values for the Maximum Height (MAXH), Middle Breadth (MIDB), and Dorsal Articular Facet Length (DAFL) calcaneal measurement variables are larger in the Black South African population (Bidmos and Asala, 2005) than in the Thai 
population. The mean values for the Maximum Length (MAXL), Cuboidal Facet Height (CFH), Body Height (BH), Minimum Breadth (MINB), Load Arm Length (LAL), and Dorsal Articular Facet Breadth (DAFB) calcaneal measurement variables are larger in the Thai population than in the Black South African population (Bidmos and Asala, 2005). The MAXL, MAXH, and BH calcaneal measurement variables have p-values greater than $0.005(p>0.005)$, which means that there is no statistically significant difference between the Thai population and the Black South African population (Bidmos and Asala, 2005). All other female calcaneal measurement variables (Cuboidal Facet Height, Minimum Breadth, Load Arm Length, Middle Breadth, Dorsal Articular Facet Breadth, and Dorsal Articular Facet Length) have a p-value of 0.000, indicating a statistically significant difference in these calcaneal measurement variable values between the Thai population and the Black South African population (Bidmos and Asala, 2005) (Table 4.15).

The Thai measurements were also compared with a White South African population (Bidmos, 2006) using descriptive statistics. The study by Bidmos (2006) utilized 85 complete skeletons $\left(\mathrm{N}_{\text {individuals }}=85 ; \mathrm{N}_{\text {male }}=41, \mathrm{~N}_{\text {female }}=44\right)$ from the Raymond A. Dart Collection of Human Skeletons, in order to create regression equations for living stature estimation from the calcaneus. The mean values (in millimeters) of each calcaneal measurement variable were compared between the Thai population and a White South African population (Bidmos, 2006) (Table 4.16). 
Table 4.16 Comparison of male and female Thai measurements of the calcaneus to a White South African population (Bidmos, 2006).

\begin{tabular}{|c|c|c|c|c|c|c|c|c|}
\hline \multirow{2}{*}{$\begin{array}{l} \\
\text { Measurement } \\
\text { variable }\end{array}$} & \multicolumn{3}{|c|}{$\begin{array}{l}\text { Current Study } \\
\text { Thai }\end{array}$} & \multicolumn{3}{|c|}{$\begin{array}{l}\text { Bidmos (2006) } \\
\text { White South African }\end{array}$} & \multirow[b]{2}{*}{$t$-value } & \multirow[b]{2}{*}{$p$-value } \\
\hline & & & & & & & & \\
\hline \multirow[b]{2}{*}{ Male } & $N$ & Mean & SD & $N$ & Mean & SD & & \\
\hline & & & & & & & & \\
\hline \multirow{9}{*}{$\begin{array}{l}\text { MAXL } \\
\text { MAXH } \\
\text { CFH }\end{array}$} & 86 & 80.49 & 4.72 & 41 & & 5.40 & -3.58 & $0.001 *$ \\
\hline & 86 & 43.62 & 3.56 & 41 & 84.02 & 4.11 & -5.41 & $0.000 *$ \\
\hline & 86 & 26.79 & 2.19 & 41 & 47.67 & 2.21 & 3.17 & $0.002 *$ \\
\hline & 86 & 39.06 & 2.91 & 41 & 25.46 & 2.79 & -0.16 & 0.870 \\
\hline & 86 & 25.71 & 1.88 & 41 & 39.15 & 2.23 & -2.11 & 0.039 \\
\hline & 86 & 48.68 & 2.82 & 41 & 26.56 & 3.80 & 2.23 & 0.030 \\
\hline & 86 & 40.97 & 2.21 & 41 & 47.20 & 2.29 & -2.63 & 0.010 \\
\hline & 86 & 29.37 & 1.88 & 41 & 42.10 & 1.91 & 16.28 & $0.000 *$ \\
\hline & 86 & 29.25 & 1.95 & 41 & $\begin{array}{l}23.50 \\
31.40\end{array}$ & 2.63 & -4.66 & $0.000 *$ \\
\hline
\end{tabular}

Female

$\begin{array}{lllllllll}\text { MAXL } & 87 & 73.44 & 4.26 & 44 & 75.45 & 4.08 & -2.62 & 0.010 \\ \text { MAXH } & 87 & 39.53 & 3.57 & 44 & 42.95 & 3.37 & -5.37 & 0.000^{*} \\ \text { CFH } & 87 & 24.29 & 1.82 & 44 & 22.88 & 1.96 & 3.98 & 0.000^{*} \\ \text { BH } & 87 & 34.29 & 2.45 & 44 & 35.30 & 3.01 & -1.93 & 0.058 \\ \text { MINB } & 87 & 23.48 & 1.90 & 44 & 23.28 & 2.15 & 0.53 & 0.601 \\ \text { LAL } & 87 & 44.15 & 2.82 & 44 & 41.96 & 2.82 & 4.20 & 0.000^{*} \\ \text { MIDB } & 87 & 37.08 & 2.16 & 44 & 37.66 & 2.29 & -1.40 & 0.166 \\ \text { DAFB } & 87 & 26.02 & 1.76 & 44 & 19.96 & 1.77 & 20.40 & 0.000^{*} \\ \text { DAFL } & 87 & 25.80 & 1.78 & 44 & 27.31 & 1.52 & -4.62 & 0.000^{*}\end{array}$

* $p<0.005$

For males, the mean Maximum Length (MAXL), Maximum Height (MAXH), Body Height (BH), Minimum Breadth (MINB), Middle Breadth (MIDB), and Dorsal Articular Facet Length (DAFL) calcaneal measurement variables were larger in the White 
South African population (Bidmos, 2006) than in the Thai population. In contrast, the mean values of the Cuboidal Facet Height (CFH) Load Arm Length (LAL), and Dorsal Articular Facet Breadth (DAFB) calcaneal measurement variables were larger in the Thai population than in the White South African population (Bidmos, 2006). The male MAXL, MAXH, CFH, DAFB, and DAFL calcaneal measurement variables had p-values that were less than $0.005(p<0.005)$ which means that there is a statistically significant difference in these calcaneal measurement variable values between the Thai population and the White South African population (Bidmos, 2006). The male BH, MINB, and LAL calcaneal measurement variables had $p$-values that were greater than $0.005(p>0.005)$, which means that there is no statistically significant difference in these calcaneal measurement variable values between the Thai and White South African populations (Bidmos, 2006). The $\mathrm{p}$-value of the male MIDB calcaneal measurement variable was $\mathrm{p}=$ 0.010. While there was no "statistically significant" difference in the male MIDB calcaneal measurement variable between Thai males and White South African males (Bidmos, 2006), this measurement variable shows a higher degree of difference between these populations than the male $\mathrm{BH}, \mathrm{MINB}$, and LAL calcaneal measurement variables. For females, the mean values for the Maximum Length (MAXL), Maximum Height (MAXH), Body Height (BH), Middle Breadth (MIDB), and Dorsal Articular Length (DAFL) calcaneal measurement variables were larger in the White South African population (Bidmos, 2006) than in the Thai population. The Cuboidal Facet Height (CFH), Minimum Breadth (MINB), Load Arm Length (LAL), and Dorsal Articular Facet Breadth (DAFB) calcaneal measurement variable values were larger in the Thai population. The female MAXH, CFH, LAL, DAFB, and DAFL calcaneal measurement 
variables had $\mathrm{p}$-values of less than $0.005(p \leq 0.005)$ which means that there is a statistically significant difference in these calcaneal measurement variables between the Thai population and the White South African population (Bidmos, 2006). The female $\mathrm{BH}, \mathrm{MINB}$, and MIDB calcaneal measurement variables had $\mathrm{p}$-values that were greater than $0.005(p>0.005)$, which means there is no statistically significant difference in these calcaneal measurement variables between the Thai and White South African population (Bidmos, 2006) The p-value of the female MAXL calcaneal measurement variable was $p=0.010$. While there was no "statistically significant" difference in the female MAXL calcaneal measurement variable between Thai females and White South African females (Bidmos, 2006), this measurement variable shows a higher degree of difference between these populations than the female BH, MINB, and MIDB calcaneal measurement variables.

The Thai data were also compared to the results from Holland (1995), who utilized 100 tali $\left(\mathrm{N}_{\text {individuals }}=100\right)$ from the Hamann-Todd Collection at the Cleveland Museum of Natural History in order to derive regression equations for living stature estimation. The results of the study by Holland (1995) provide regression equations for Maximum Length of the Talus (MTAL) for Black males and Black females $\left(\mathrm{N}_{\text {individuals }}=\right.$ $50 ; \mathrm{N}_{\text {male }}=25, \mathrm{~N}_{\text {female }}=25$ ). The mean values (in millimeters) of each talar measurement variable were compared between the Thai population and the Black American population (Holland, 1995) using descriptive statistics (Table 4.17). 
Table 4.17 Comparison of male and female Thai measurements of the talus to a Black American population (Holland, 1995).

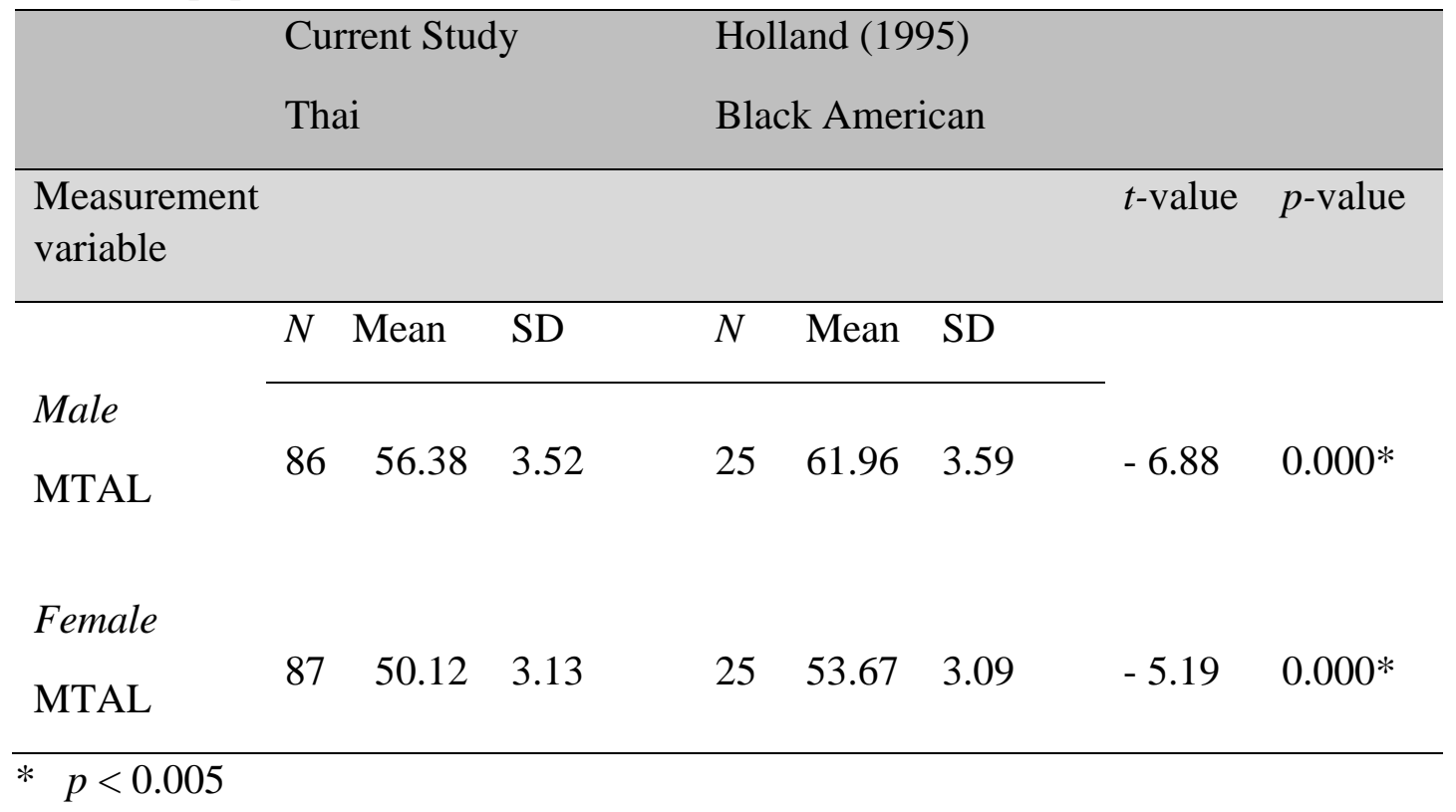

The mean MTAL talar measurement variable value was larger for both males and females in the Black American population (Holland, 1995) than in the Thai population. The p-values for both males and females was 0.000 , which indicates that there is a statistically significant difference in the mean MTAL talar measurement variable values between the Thai population and the Black American population (Holland, 1995) (Table 4.17). 


\section{CHAPTER 5: DISCUSSION}

\subsection{Context of the Current Research}

When unknown skeletal remains are recovered, the goal of the forensic anthropologist is to assist in the identification through the creation of a biological profile. Previous authors have shown that osteometric differences exist between different populations and have suggested that methods derived from one specific population should not be used for other populations (Bidmos, 2006; Bidmos and Asala, 2005; Holland, 1995; King et al., 1998; Lundy, 1983; Steyn and Işcan, 1997; Trotter and Gleser, 1958).

There is an urgent need for the development of population-specific osteometric methodologies for contemporary Thai populations. The impact of climate change is estimated to be particularly severe in Thailand; recent studies have shown that the effects of climate change will continue to impact Thailand as flooding and natural disasters will multiply in both frequency and severity, which will have negative impacts in terms of death and destruction (Arunrat and Pumijumnong, 2015; Jinsart et al., 2014; Jothityangkoon et al., 2013; Thavorntam and Tantemsapya, 2013; Wang et al., 2014). However, these methodologies must prove high levels of reliability, accuracy, and precision to be admissible in a court of law (Berg et al., 2007; Christensen and Crowder, 2009; Lesciotto, 2015).

Research has shown that preservationally favoured and/or complete skeletal elements, such as the calcaneus and the talus, are suitable for the identification of unknown human remains, and are often recovered at forensic scenes as they are protected inside shoes (Bidmos and Asala, 2003; Pickering, 1986; Saul and Saul, 2005; Scott et al., 2017). Studies have shown that the calcaneus and the talus can be used for the estimation sex 
(Abd-elaleem et al., 2012; Barrett et al., 2001; Bidmos, 2006; Bidmos and Asala 2003, 2004, 2005; Bidmos and Dayal, 2003, 2004; DiMichele and Spradley, 2012; Ekizoglu et al., 2017; Gualdi-Russo, 2007; Holland, 1995; Introna et al., 1997; Kim et al., 2013; Lee et al., 2012; Mahakkanukrauh et al., 2014; Moore et al., 2016; Murphy, 2002a,b, 2005; Nathena et al., 2017; Otong et al., 2016; Peckmann et al., 2015a,b; Riepert et al., 1996; Scott et al., 2017; Silva, 1995; Steele, 1976; Wilbur, 1998; Zakaria et al., 2010; Zhang et al., 2016). However, there are limited published studies, which investigate the accuracy of the calcaneus and the talus for the estimation of stature (Bidmos and Asala, 2005; Bidmos, 2006; Holland, 1995; Pablos et al., 2013). Thus far, the calcaneus has been used for living stature estimation in a Black South African population (Bidmos and Asala, 2005) and White South African population (Bidmos, 2006), and the talus has been used for living stature estimation in a Black American population (Holland, 1995). To date, neither the calcaneus nor the talus has been used to estimate stature in an Asian population, specifically a contemporary Thai population. The current research study aims to fill this gap in the literature.

The current research study focuses on nine measurements of the calcaneus (Maximum Length, Maximum Height, Cuboidal Facet Height, Body Height, Minimum Breadth, Load Arm Length, Middle Breadth, Dorsal Articular Facet Breadth, and Dorsal Articular Facet Length) and one measurement of the talus (Maximum Length of the Talus) to establish an accurate method for estimating living stature in a contemporary Thai population. The objectives of this research are to:

1. investigate whether regression equations for the calcaneus, which were developed on a Black South African population (Bidmos and Asala, 2005), and regression equations 
for the talus, which were developed on a Black American population (Holland, 1995), can accurately predict the living stature of individuals from a contemporary Thai population.

2. develop population-specific simple regression and multiple regression equations for the calcaneus and the talus of the contemporary Thai population should the Black South African (Bidmos and Asala, 2005) and Black American (Holland, 1995) equations not accurately predict the living stature of the Thai individuals.

3. compare the results from the contemporary Thai population to other populations.

\subsection{Analysis of Sexual Dimorphism in the Thai Population}

The results of the current research show that all measurement variables of the calcaneus and the talus exhibit sexual dimorphism in the contemporary Thai population. These results are consistent with those of other researchers who have shown that the calcaneus and the talus are sexually dimorphic (Abd-elaleem et al., 2012; Barrett et al., 2001; Bidmos, 2006; Bidmos and Asala 2003, 2004, 2005; Bidmos and Dayal, 2003, 2004; DiMichele and Spradley, 2012; Ekizoglu et al., 2017; Gualdi-Russo, 2007;

Holland, 1995; Introna et al., 1997; Kim et al., 2013; Lee et al., 2012; Mahakkanukrauh et al., 2014; Moore et al., 2016; Murphy, 2002a,b, 2005; Nathena et al., 2017; Otong et al., 2016; Peckmann et al., 2015a,b; Riepert et al., 1996; Scott et al., 2017; Silva, 1995; Steele, 1976; Wilbur, 1998; Zakaria et al., 2010; Zhang et al., 2016).

For the calcaneus, all measurement variables (i.e. MAXL, MAXH, BH, CFH, MINB, LAL, MIDB, DAFL, DAFB) were larger in contemporary Thai males than in contemporary Thai females (Table 4.2). In the current research, the most dimorphic 
measurement variable of the calcaneus was the MAXL measurement variable, with a mean difference of $7.05 \mathrm{~mm}$ between the maximum length of the contemporary Thai male calcanei and the contemporary Thai female calcanei. These results are consistent with the published literature (Bidmos, 2006; Bidmos and Asala 2003, 2004, 2005; Ekizoglu et al., 2017; Gualdi-Russo, 2007; Holland, 1995; Introna et al., 1997; Kim et al., 2013; Moore et al., 2016; Murphy, 2002a; Nathena et al., 2017; Peckmann et al., 2015a; Riepert et al., 1996; Scott et al., 2017; Silva, 1995; Steele, 1976; Wilbur, 1998; Zakaria et al., 2010; Zhang et al., 2016). Overall, the literature shows that the maximum length of the calcaneus (i.e. the MAXL measurement variable) exhibits more dimorphism than any other measurement variable of the calcaneus, with mean differences between males and females ranging from $5.60 \mathrm{~mm}$ to $10.67 \mathrm{~mm}$. Table 5.1 shows the mean differences between the male maximum length of the calcaneus (MAXL) measurement variable and the female maximum length of the calcaneus (MAXL) measurement variable for different studies, and is organized from most dimorphic to least dimorphic by the mean difference in relation to the size of the calcaneus (i.e. \% difference). DiMichele and Spradley (2012) however, found that the posterior circumference (PCF) of the calcaneus, a measurement variable newly introduced for their research study, was the most dimorphic measurement variable of the calcaneus and MAXL was the second most dimorphic measurement variable. 
Table 5.1 Comparison of the mean difference between the male maximum length of the calcaneus (MAXL) measurement variable and the female maximum length of the calcaneus (MAXL) measurement variable between different studies.

\begin{tabular}{|c|c|c|c|}
\hline Investigator & Population & $\%$ difference ${ }^{a}$ & $\begin{array}{l}\text { Mean difference } \\
(\mathrm{mm})\end{array}$ \\
\hline Zakaria et al. (2010) & Egyptian & 13.5 & 10.67 \\
\hline Ekizoglu et al. (2017) & Turkish & 12.1 & 9.79 \\
\hline Murphy (2002a) & $\begin{array}{l}\text { Prehistoric New } \\
\text { Zealand Polynesian }\end{array}$ & 11.9 & 8.99 \\
\hline Bidmos and Asala (2003) & White South African & 11.1 & 8.91 \\
\hline Bidmos (2006) & White South African & 10.8 & 8.57 \\
\hline Peckmann et al. (2015a) & Greek & 10.7 & 8.27 \\
\hline Wilbur (1998) & $\begin{array}{l}\text { Prehistoric West } \\
\text { Central Illinois }\end{array}$ & 10.6 & 7.90 \\
\hline Gualdi-Russo (2007) & Italian & 10.4 & 8.25 \\
\hline Zhang et al. (2016) & Chinese & 9.9 & 6.78 \\
\hline Moore et al. (2016) & Colombian & 9.4 & 6.78 \\
\hline Current research study & Thai & 9.2 & 7.05 \\
\hline Silva (1995) & Portuguese & 9.2 & 6.95 \\
\hline Riepert et al. (1996) & German & 9.1 & 7.80 \\
\hline Kim et al. (2013) & Korean & 8.9 & 6.70 \\
\hline Steele (1976) & Black American & 8.7 & 6.90 \\
\hline Introna et al. (1997) & Southern Italian & 8.7 & 6.60 \\
\hline Scott et al. (2017) & Thai & 8.4 & 6.47 \\
\hline Bidmos and Asala (2005) & Black South African & 8.3 & 6.33 \\
\hline Bidmos and Asala (2004) & Black South African & 8.0 & 6.14 \\
\hline Nathena et al. (2017) & Cretan & 7.8 & 6.05 \\
\hline Steele (1976) & White American & 7.2 & 5.60 \\
\hline
\end{tabular}

a The mean difference in relation to the size of the calcaneus 
For the talus, the MTAL measurement variable was larger in contemporary Thai males than in contemporary Thai females (Table 4.2). The mean difference in the MTAL measurement variable between contemporary Thai male tali and contemporary Thai female tali was $6.36 \mathrm{~mm}$. This result is consistent with the existing published literature (Abd-elaleem et al., 2012; Barrett et al., 2001; Bidmos and Dayal, 2003, 2004; GualdiRusso, 2007; Holland, 1995; Lee et al., 2012; Mahakkanukrauh et al., 2014; Murphy, 2002b; Otong et al., 2016; Peckmann et al., 2015b; Silva, 1995; Steele, 1976; Wilbur, 1998).

For the current research study, only the maximum length of the talus (i.e. MTAL measurement variable) was examined following the protocol of other stature estimation literature (i.e. Holland, 1995). However, an earlier study by Mahakkanukrauh et al. (2014) examined sexual dimorphism of the talus using the same skeletal collection as the current research study (i.e. the Chiang Mai Skeletal Collection). Mahakkanukrauh et al. (2014) examined ten talar measurements, including: maximum talar length (MaxLg), maximum talar breadth (MaxBr), maximum talar height (MaxHt), maximum trochlear length (MaxTrLg), maximum trochlear breadth (MaxTrBr), maximum length of the inferior articular surface (MaxIASLg), maximum breadth of the inferior articular surface (MaxIASBr), minimum inferior interarticular distance (MinIID), maximum lateral malleolar surface height (MaxLMSHt), and minimum interarticular distance across the neck (MinIDNk). Of these, Mahakkanukrauh and colleagues (2014) found that the maximum length of the talus was the most sexually dimorphism measurement variable. As Mahakkanukrauh and colleagues (2014) utilized the same skeletal collection as the current research study, it can be suggested that the maximum length of the talus (i.e. the 
MTAL measurement variable) is the most sexually dimorphic measurement variable of the talus in a contemporary Thai population. This is consistent with the existing published literature, which has consistently shown that the maximum length of the talus exhibits more dimorphism than other measurement variables of the talus, with mean differences between male and female maximum talar length ranging from $3.71 \mathrm{~mm}$ to $8.29 \mathrm{~mm}$. Table 5.2 shows the mean difference between the male maximum length of the talus (MTAL) measurement variable and the female maximum length of the talus (MTAL) measurement variable for different studies, and is organized from most dimorphic to least dimorphic by the mean difference in relation to the size of the talus (i.e. \% difference). Abd-elhakim and colleagues (2012) however, found that the neck length (NL) of the talus was the most dimorphic measurement variable of the talus in an Egyptian population. The NL measurement is defined as the distance between the most distant point of the navicular articular facet to the anterior end of the middle sagittal curvature of the trochlea (Abd-elhakim et al., 2012; Martin and Knußmann, 1988). Future studies should utilize the NL measurement variable when assessing sexual dimorphism of the talus in other populations. 
Table 5.2 Comparison of the mean difference between the male maximum length of the talus (MTAL) measurement variable and the female maximum length of the talus (MTAL) measurement variable between different studies.

\begin{tabular}{|c|c|c|c|}
\hline Investigator & Population & $\%$ difference $^{\mathrm{a}}$ & $\begin{array}{c}\text { Mean difference } \\
(\mathrm{mm})\end{array}$ \\
\hline Holland (1995) & Black American & 14.3 & 8.29 \\
\hline Peckmann et al. (2015b) & Greek & 12.4 & 7.10 \\
\hline Gualdi-Russo (2007) & Northern Italian & 13.0 & 6.85 \\
\hline Current research study & Thai & 11.9 & 6.36 \\
\hline $\begin{array}{l}\text { Mahakkanukrauh et al. } \\
\text { (2014) }\end{array}$ & Thai & 11.5 & 6.13 \\
\hline Steele (1976) & Black American & 11.5 & 6.00 \\
\hline Wilbur (1998) & $\begin{array}{l}\text { Prehistoric West } \\
\text { Central Illinois }\end{array}$ & 10.9 & 5.40 \\
\hline Steele (1976) & White American & 10.7 & 5.60 \\
\hline Barrett et al. (2001) & $\begin{array}{l}\text { Prehistoric Native } \\
\text { American }\end{array}$ & 10.6 & 5.77 \\
\hline Murphy (2002b) & $\begin{array}{l}\text { Prehistoric New } \\
\text { Zealand Polynesian }\end{array}$ & 10.4 & 5.24 \\
\hline Silva (1995) & Portuguese & 10.3 & 5.23 \\
\hline Bidmos and Dayal (2004) & Black South African & 9.3 & 4.61 \\
\hline Holland (1995) & White American & 9.2 & 5.42 \\
\hline Bidmos and Dayal (2003) & White South African & 8.4 & 4.50 \\
\hline Lee et al. (2012) & Korean & 6.9 & 3.71 \\
\hline
\end{tabular}

${ }^{\mathrm{a}}$ The mean difference in relation to the size of the talus.

The size and shape of the calcaneus and the talus may vary between sexes and populations due to differences in genetics, socioeconomic status, and habitual or occupational behaviours (Abd-elhakim, 2012; Ekizoglu et al., 2017; Kim et al., 2013; Lee et al., 2012; Mahakkanukrauh et al., 2014). These influences may explain why certain 
populations show larger mean differences, or exhibit more sexual dimorphism, in the MAXL and MTAL measurement variables than other populations.

Genetic factors and the genetically controlled release of hormones have been shown to influence pubertal timing, skeletal development, and growth rates in males and females (Bailey and Garn, 1986; Gajdos et al., 2009, 2010; Humphrey, 1998). Growth hormones (GH and IGH-1), and sex and adrenal steroids, are responsible for the timing of growth spurts and attainment of maturity. Females experience growth spurts and maturation, on average, two years prior to males (Eckes and Trautner, 2000; Humphrey, 1998; Loesch et al., 1995). The earlier commencement of puberty for females is associated with an earlier secretion of estrogen, the effect of which is to increase bone maturation at the cost of reducing pubertal gain in stature (Bourguignon, 1988). As a result, males reach a greater overall size prior to their final growth spurt in puberty than females (Loesch et al., 1995; Parent et al., 2003). Furthermore, the genetic regulation of pubertal timing, and thus skeletal development and growth rates, has been shown to vary among populations (Gajdos et al., 2009, 2010; Parent et al., 2003). For example, Stavrou and colleagues (2002) found that two polymorphisms of the estrogen receptor a gene were associated with delayed menarche in Greek adolescent females. However, in a study of Japanese adolescent girls, Gorai and colleagues (2003) found that menarcheal age was not associated with the estrogen receptor a gene, but rather was linked to the A2 polymorphism of CYP17 gene. These findings suggest that the involvement of genetics in controlling female pubertal timing is variable between populations.

The mean age at menarche in a contemporary Thai population is 11.8 years (Noipayak et al., 2017). As earlier commencement of menarche is associated with a 
reduction in female stature and thus an increase in dimorphism between the sexes, the current author suggests that populations that exhibit less sexual dimorphism of the calcaneus and the talus may demonstrate a mean age at menarche that is later than that which occurs in the contemporary Thai population. The mean age at menarche for populations that exhibit less sexual dimorphism of the calcaneus and the talus when compared to the contemporary Thai population (according to Table 5.1 and Table 5.2) is as follows: 11.86 years (Portuguese; Reis et al., 2016), 15.0 (historic Black American, Trussell and Steckle, 1978), 12.70 (Korean; Lee et al., 2016), 12.17 (Southern Italian; De Sanctis et al., 2014), 12.8 (German; Bau et al., 2009), 14.11 (Black South African; Anderson, 2015), and 12.50 (White American; Reagan et al., 2012). As these populations show a later mean age at menarche than the contemporary Thai population, it is possible that they did not experience the same reduction of pubertal gain in stature as shown in the contemporary Thai population.

Socioeconomic status has also been shown to influence skeletal development and overall growth in males and females, thus contributing to expressions of sexual dimorphism (Danubio and Sanna, 2008; Figueroa-Soto, 2012; Parent et al., 2003). Previous studies have demonstrated that populations exposed to environmental stressors, such as low socioeconomic status, will exhibit less sexual dimorphism than populations not exposed to such stressors (Figueroa-Soto, 2012; Hamilton, 1975; Malina et al., 1981; Rickland and Tobias, 1986; Stini, 1969, 1972). The Female Buffering Hypothesis for example, suggests that the growth and development of males in a low socioeconomic status is more affected than that of females, in the same environment, during times of nutritional shortage and poverty (Frayer and Wolpoff, 1985; Stinson, 1985). In 1951, 
Greulich found that food deprivation and poverty retarded the skeletal development of Guamanian males (i.e. low socioeconomic status) during the Japanese occupation, which resulted in females being larger and more robust than males in this population. These results are supported by Stini (1969) who also found that the long-term effects of malnutrition (i.e. growth retardation) in a Heliconia population were more pronounced in males than in females, thus reducing the degree of sexual dimorphism.

The Chiang Mai Skeletal Collection, utilized for the current research study, consists of individuals from Chiang Mai and adjacent villages in northern Thailand with recorded occupations consistent with middle and low socioeconomic status, including farmers and public servants (King et al., 1998; Traithepchanapai et al., 2016). While only $10.5 \%$ of the total contemporary Thai population live below national poverty lines, poverty in Thailand does exist as a mainly rural phenomenon. As of 2014 , over $80 \%$ of the country's poor live in rural areas, and pockets of poverty remain in the North and Northeast regions (World Bank, 2017). Therefore, the contemporary Thai population is of lower-middle-socioeconomic status. The current author suggests that populations with a higher socioeconomic status than the contemporary Thai population, will exhibit more sexual dimorphism.

The World Bank (2017) lists Italy, Greece, and Central Europe as high-income countries, and lists Turkey, South Africa, China, and Colombia as upper-middle-income countries. These populations are likely to have improved nutrition, clean water, and sanitation, increased economic activity (i.e. more job opportunities), and greater access to administrative structures and services such as health care and education. As a result, these populations will have better overall health, growth, and development, which may explain 
the higher expression of sexual dimorphism in these populations in comparison to populations of lower socioeconomic status, such as the contemporary Thai population (Figueroa-Soto, 2012; Hamilton, 1975; Leon, 2008; Malina et al., 1981; Paciorek et al., 2013; Rickland and Tobias, 1986; Stini, 1969, 1972).

The Egyptian population (Zakaria et al., 2010) is representative of an urban population. Research has shown that children living in cities are taller and have enhanced growth and development in comparison to their rural counterparts in many low-middleincome countries (Black et al., 2013; Fox and Heaton, 2012; Heaton and Forste, 2003; Paciorek et al., 2013; Panter-Brick et al., 1996; Peckmann et al., 2017; Smith et al., 2005). Panter-Brick et al. (1996) for example compared the heights of children from Nepal and Pakistan living in rural villages with those living in an urban environment, and found that children living in urban environments showed better overall growth and development than children living in rural villages. Overall, cities offer access to services (e.g. healthcare and education) and infrastructure (e.g. sanitation, clean water), which may increase the quality of life for those in a lower socioeconomic bracket (Fox and Heaton, 2012; Paciorek et al., 2013; Panter-Brick et al., 1996; Peckmann et al., 2017). Therefore, it is likely that the Egyptian population (Zakaria et al., 2010) had better overall health, growth, and development than the contemporary Thai population, which may explain the higher expression of sexual dimorphism in this population, despite the similarities in cited socioeconomic status (Figueroa-Soto, 2012; Hamilton, 1975; Malina et al., 1981; Rickland and Tobias, 1986; Stini, 1969, 1972).

Sexual dimorphism may also be influenced by divisions of labour. This hypothesis can be traced back to Darwin (1874), who suggested that sexual dimorphism 
evolves through natural selection in species where males and females occupy different ecological niches, or have different foraging strategies. Human societies are characterized by marked sexual divisions of labour in subsistence strategies, which is the equivalent of animal foraging strategies (Murdock and Provost, 1973). This hypothesis has been developed in paleoanthropology; Brace and Ryan (1980) and Frayer $(1980,1981)$ suggested that changes in male and female subsistence activities may explain the decline in sexual dimorphism since the Late Paleolithic. The results of more recent studies support this; Carlson and colleagues (2007) for example studied Australian aborigine hunter-gatherer groups and found that sexual dimorphism was greater in groups, which practiced a division of labour. Groups that had adopted agriculture, and in which both males and females were involved in similar food production and preparation tasks, exhibited a low degree of sexual dimorphism (Carlson et al., 2007). Holden and Mace (1999) conducted a similar study and found that when females were involved with food production and preparation, they were taller and more robust, and thus sexual dimorphism at the population level was reduced. Overall, the published literature has demonstrated that divisions of labour vary across populations, which may explain differences in the expression of sexual dimorphism between contemporary populations for the calcaneus and the talus (Abd-elhakim et al., 2012; Carlson et al., 2007; Ekizoglu et al., 2017; Holden and Mace, 1999; Nathena et al., 2017; Scott et al., 2017).

The individuals represented in the current research study are from the northern region of Thailand, an area in which rice farming is popular (Arunrat and Pumijumnong, 2015; Isvilanonda and Bunyasiri, 2009). Rice farming involves the navigation of uneven terrain and hard manual labour and is traditionally considered men's work. While women 
are involved in some aspects of rice production, their participation is limited to peak seasons of land preparation and they are often only assigned to production tasks when time constraints are imposed (e.g. by weather) or when the need is dire (Coyle and Kwong, 2000). Furthermore, women are often excluded when machines replace physical labour and the status of the job is raised. Coyle and Kwong (2000) cite that traditionally, women were responsible for grain transportation, but contemporary Thai men have since taken over this task with the introduction of the truck, which bestows prestige and mobility on the operator. Overall, contemporary Thai women are more involved in child rearing, housework, and entrepreneurial activities (e.g. selling surplus rice or vegetables at a local market or in their home) than contemporary Thai men, resulting in a division of labour between the sexes, and thus increasing sexual dimorphism at the population level. In contrast, Cretan females of rural regions are more involved in manual labour; Nathena and colleagues (2017) compared expressions of sexual dimorphism of the calcaneus between a Cretan population and a Greek population and found that the increased sexual dimorphism in the Greek population may be the result of differences in female activity patterns between the two populations. The Greek population can be described as "urban", whereas the Cretan population can be described as "rural". Cretan females of rural regions tend to be more involved in manual labour, thus increasing their bone size and decreasing the level of sexual dimorphism in the Cretan population (Nathena et al., 2017). Overall, divisions of labour between males and females are different between the contemporary Thai and Cretan (Nathena et al., 2017) populations, with the contemporary Thai population showing a more sexually dimorphic division of labour in which males are more involved with food production and manual labour than 
contemporary Thai females (Coyle and Kwong, 2000). Therefore, differences in divisions of labour may be responsible for differences in the expression of sexual dimorphism between the current research study and other population studies.

Lastly, some studies (Barrett et al., 2001; Murphy, 2002a,b, 2005; Wilbur, 1998) have utilized prehistoric skeletal samples to examine sexual dimorphism; thus, secular change may also be a reason for differences in the expression of sexual dimorphism between populations. Secular change in a non-genetic response to sociocultural and environmental pressures, which causes an increase in overall body dimensions and earlier onset of menarche over several decades or generations (Danubio and Sanna, 2008; Roche, 1979). Positive secular change (i.e. increases in body dimensions and earlier onset of menarche) can be attributed to enhanced population health (e.g. the creation of modern medicine or the advancement of medical technologies for treatment and diagnosis), improved nutrition, and an increase in quality of living conditions (Albanese, 2010; Chan, 2011; Danubio and Sanna, 2008). Malina (1981) for example, showed that improvements in sociocultural and environmental pressures such as the improvement of water and sanitation, or the elimination of infectious disease over time may lead to increased maturation/earlier onset of menarche, which has been shown to influence the expression of sexual dimorphism at the population level (Bourguignon, 1988; Gajdos et al., 2004; Loesch et al., 1995; Parent et al., 2003).

The individuals examined in the current research study represent a contemporary Thai population; these individuals lived during the $20^{\text {th }}$ and $21^{\text {st }}$ centuries and have had access to more contemporary diets and medical treatments. In contrast, Barrett and colleagues (2001) examined prehistoric Ohio Valley Native Americans from three Late 
Archaic hunting-gathering populations, Murphy (2002a,b) examined New Zealand Polynesian skeletal remains from the prehistoric (pre-European settlement) period, and Wilbur (1998) examined prehistoric Native Americans from West-Central Illinois, spanning the Middle Woodland to Mississippian periods (AD 1-1100). As prehistoric Native American populations began to develop and depend upon agriculture, populations would have aggregated into sedentary settlements (Boyd, 1996). Similarly, prehistoric New Zealand Polynesians were settling into transient, short-term villages and specialized sites for subsistence activities (Kinaston et al., 2013). These types of settlements would have had poor sanitation and waste disposal, factors that increased the frequency of infection and disease (e.g. diarrheal infections and iron deficiency anemia), which would have been exacerbated by the fact that prehistoric populations did not have access to modern medicine or medical technologies for treatment (Boyd, 1996; Lewis, 1998). As a result, these populations would have had poorer population health, higher susceptibility to disease and lack of modern medicine/medical care, higher nutritional stress, and lower quality of living conditions than the contemporary Thai population (Barrett et al., 2001; Murphy, 2002a,b; Wilbur, 1998). This may be one reason that the expression of sexual dimorphism observed in the prehistoric Native American (Barrett et al., 2001), prehistoric New Zealand Polynesian (Murphy, 2002b), and prehistoric West-Central Illinois (Wilbur, 1998) populations is lower than that observed in the contemporary Thai population.

Table 5.1 however, indicates that the amount of sexual dimorphism expressed in the calcaneus is higher in the prehistoric New Zealand Polynesian (Murphy, 2002b), and prehistoric West-Central Illinois (Wilbur, 1998) populations than in the contemporary Thai population. This may be because the calcaneus is a weight-bearing bone, and 
subsistence strategies associated with hunter-gatherer populations may have placed strain on this bone, causing it to become more robust. In most hunter-gatherer and foraging societies, males are often responsible for large game hunting that requires high terrestrial mobility and habitual loading associated with tracking, hunting, and carrying large game animals (Marlowe, 2007; Ruff, 2008; Stock and Macintosh, 2016). These activities are likely to have the greatest influence on the morphology of weight-bearing bones among prehistoric populations and may have resulted in larger male calcanei (Stock and Macintonish, 2016). Therefore, the subsistence strategies of the prehistoric New Zealand Polynesian (Murphy, 2002b) and prehistoric West-Central Illinois (Wilbur, 1998) populations may have caused a higher expression of sexual dimorphism in the weightbearing calcaneus than that exhibited in the contemporary Thai population, despite the influence of positive secular change.

\subsection{Analysis of Observer Error and Reproducibility}

In recent years, and particularly since the 1993 Supreme Court Daubert decision that addressed the standard of admissibility for expert testimony, the forensic community has become increasingly concerned with the reliability of methods from which scientific conclusions are drawn (Berg et al., 2007; Lesciotto, 2015). Reliability can be defined as "how frequently a method yields the same results between successive trials" (i.e. its repeatability) (Berg et al., 2007: 1020) In forensic anthropology, methods and measurements must be well defined so that future researchers are able to accurately repeat the measurements. Without accurate repeatability, methods and measurements are considered unreliable (Berg et al., 2007). To ensure high accuracy and repeatability of the 
calcaneus and the talus measurements, the current research study calculates intra-observer and inter-observer error rates using paired t-tests to evaluate error bias, following the protocol of Holland (1995), and also utilizes the concordance correlation of reproducibility method (Lin, 1989), following the protocol of Bidmos and Asala (2005).

For the current research study, intra-observer error was assessed by calculating the error associated with repeated measurements of calcaneal and talar measurement variables by the current author. When examining the calcaneal and talar measurements, the results showed that accuracy and repeatability were maintained for all variables when the current author remeasured a subsample at a different time, which indicates consistency between the first and second data sets. However, the male DAFB measurement variable and the female $\mathrm{CFH}, \mathrm{MINB}$, and DAFB measurement variables showed concordance correlation coefficient values below the internationally accepted standard range. This indicates that these measurement variables are not highly reproducible, and may not be accurately repeated in future studies. Therefore, these measurements should be used with caution during future studies and practical application.

Inter-observer error was assessed by calculating the error associated with repeated measurements of calcaneal and talar variables by two different observers (i.e. the current author and a research assistant). The results showed no statistically significant differences between the current author and the research assistant, which indicates that all measurement variables were accurately repeated between observers. However, the male DAFB and the female BH measurement variables showed concordance correlation coefficient values below the internationally accepted standard range, which suggests that these measurement variables are not highly repeatable. The research assistant had no 
previous experience or familiarity with the measurements of the current research study. Therefore, the low concordance correlation coefficient values may have resulted from inexperience or lack of familiarity with the DAFB and $\mathrm{BH}$ measurement variables. Previous studies have shown that investigator experience and familiarity with a method may be a potential source of observer error (Berg et al., 2007). To enhance the familiarity of a user with these measurement variables, and thus the repeatability of these measurement variables, training with theoretical discussions and practical activities is recommended prior to application (Lopez et al., 2017).

Overall, the results of intra-and-inter-observer error analyses demonstrate discrepancies within or between observers for the male DAFB measurement variable and the female CFH, MINB, DAFB, and BH measurement variables. This indicates that these measurements may not be accurately repeated in future studies and as such may be considered unreliable (Berg et al., 2007). Since the 1993 Supreme Court Daubert decision and the 1994 Canadian Supreme Court Mohan decision, the forensic community has emphasized the need for anthropological research and testimony to conform to certain standards; namely the methods and techniques used during forensic assessment must be based on reliable principles and methodologies (Berg et al., 2007; Christensen, 2004; Holobinko, 2012; Lesciotto, 2015; Williams and Rogers, 2006). If the techniques (i.e. the measurements) utilized during forensic assessment are considered unreliable or are not based on reliable principles and methodologies, the credibility of the expert witness and their professional reputation could be damaged, and testimony may be considered inadmissible (Williams and Rogers, 2006). Given the results of the current research study, the current author suggests that forensic practitioners utilize the male DAFB 
measurement variable and the female $\mathrm{CFH}, \mathrm{MINB}$, DAFB, and $\mathrm{BH}$ measurement variables during anthropological assessment only when other, more reliable (i.e. those with more accurate repeatability) measurement variables are unavailable for examination, in order to best satisfy admissibility criteria.

\subsection{Simple and Multiple Regression Equations}

Previous research has demonstrated that osteometric differences exist between populations and suggests that the methods and standards derived for victim identification be population-specific (Bidmos, 2006; Bidmos and Asala, 2005; Holland, 1995; King et al., 1998; Lundy, 1983; Steyn and Işcan, 1997; Trotter and Gleser, 1952a, b, 1958). The first objective of the current research study was to investigate whether regression equations for the calcaneus and the talus that were developed on a Black South African population (Bidmos and Asala, 2005) and a Black American population (Holland, 1995) could accurately classify individuals from a contemporary Thai population. The second objective of the current research study was to develop population-specific simple regression and multiple regression equations for the calcaneus and the talus of the contemporary Thai population should the Black South African (Bidmos and Asala, 2005) and Black American (Holland, 1995) equations not accurately predict the living stature of the Thai individuals.

\subsubsection{Accuracy of the Bidmos and Asala (2005) calcaneal regression equations}

Bidmos and Asala (2005) used nine measurements of the calcaneus (i.e. MAXL, MAXH, BH, CFH, MINB, LAL, MIDB, DAL, DAFB) to derive regression equations for 
estimation of living stature in a Black South African population. They presented correlation coefficients ( $r$-values) ranging from 0.27 to 0.47 between individual calcaneal measurements and living stature (i.e. simple regression analyses) for Black South African males. The range of values obtained for Black South African females was higher than that obtained from males (from 0.35 to 0.65 ). Combinations of variables (i.e. multiple regression analyses) showed correlation coefficients ( $r$-values) that were higher than those obtained from the use of individual variables, ranging from 0.52 to 0.60 in Black South African males and from 0.72 to 0.77 in Black South African females.

To determine whether regression equations developed by Bidmos and Asala (2005) would accurately estimate living stature for a contemporary Thai population, each of the simple and multiple regression equations developed by Bidmos and Asala (2005) were tested on the Thai data. Simple regression equations using the $\mathrm{CFH}$ calcaneal measurement variable could not be tested on the Thai sample data because Bidmos and Asala (2005) did not provide simple regression equations that used the CFH calcaneal measurement variable. Overall, when the male Black South African (Bidmos and Asala, 2005) simple regression equations were applied to the contemporary Thai population correlation coefficient values ( $r$-values) decreased. The lowered accuracy rates for the Bidmos and Asala (2005) calcaneal regression formulae when applied to the contemporary Thai population suggest that regression formulae are population-specific and that there is variation in skeletal features of the calcaneus between populations. This supports the observations of previous authors that forensic anthropologists should limit the application of regression equations to the population from which they were derived 
(Bidmos, 2006; Bidmos and Asala, 2005; Holland, 1995; King et al., 1998; Lundy, 1983;

Steyn and Işcan, 1997; Trotter and Gleser, 1952a,b, 1958).

\subsubsection{Accuracy of the Holland (1995) talar regression equations}

Holland (1995) used one measurement of the talus (i.e. MTAL) to derive a simple regression equation for estimation of living stature in a Black American population. He utilized Black American individuals from the Hamann-Todd Collection at the Cleveland Museum of Natural History. These individuals are representative of a mixed temporal population, i.e. an historic Black American population and a modern early $20^{\text {th }}$ century Black American population. The population shows birth dates ranging from 1823 to 1924 and death dates ranging from 1908 to 1939 (personal communication, LM Jellema, Collections Manager, Cleveland Museum of Natural History, January 2017). Holland (1995) presented a correlation coefficient ( $r$-value) of 0.723 between MTAL and living stature (i.e. simple regression analyses), though Holland (1995) does not specify if this correlation coefficient ( $r$-value) is the same for males and females, or if this value is the average of the subgroups. Correlation coefficients ( $r$-values) were not available for males and females separately, though male and female regression equations were presented individually (Holland, 1995).

To determine whether regression equation developed by Holland (1995) would accurately estimate living stature for a contemporary Thai population, the Black American simple regression equations developed by Holland (1995) were tested on the Thai sample data. Overall, correlation coefficient values ( $r$-values) decreased from when Black American (Holland, 1995) regression equations were applied to the contemporary 
Thai population. The lowered accuracy rates for Holland (1995) talar regression formulae when applied to the contemporary Thai population suggest that regression formulae are population-specific, and that there is variation in skeletal features of the talus between populations. These results are similar to those achieved in the current research study when testing the Black South African (Bidmos and Asala, 2005) regression equations on the contemporary Thai population, and thus support the notion that forensic anthropologists should limit the application of regression equations to the population from which they were derived (Bidmos, 2006; Bidmos and Asala, 2005; Holland, 1995; King et al., 1998; Lundy, 1983; Steyn and Işcan, 1997; Trotter and Gleser, 1952a,b, 1958).

\subsubsection{Accuracy of simple and multiple regression equations for the contemporary}

\section{Thai population}

The application of the Bidmos and Asala (2005) and Holland (1995) regression equations on the contemporary Thai population demonstrated that methods derived from a specific population should not be used for other populations. Although the calcaneus and the talus have been used for stature estimation in Black American, White American, Black South African, and South African populations (Bidmos, 2006; Bidmos and Asala, 2005; Holland, 1995; Pablos et al., 2013), these bones have not yet been used for stature estimation in Thailand. Therefore, the second objective of the current research study was to develop population-specific simple regression and multiple regression equations for the calcaneus and the talus of the contemporary Thai population. 
The accuracy of stature estimation from bones using regression equations is measured by the Standard Error of the Estimate (SEE); the lower the SEE as presented by a regression equation, the more accurate is the estimate of stature. For contemporary Thai males, the lowest SEE is observed when utilizing all ten measurement variables of the calcaneus and the talus. This suggests that all measurement variables of the calcaneus and the talus should be utilized when estimating stature for contemporary Thai males, in order to provide the most accurate results. For contemporary Thai females, the lowest SEE is observed when utilizing only the MAXL calcaneal measurement variable. This suggests that utilizing combinations of measurement variables, or including the talus in the analysis, does not increase the accuracy of stature estimation in a contemporary female Thai population. Therefore, when estimating stature for contemporary Thai females, only the MAXL measurement variable should be used in order to obtain the most accurate results.

To date, there has only been one study completed on stature estimation in a contemporary Thai population (Mahakkanukrauh et al., 2011). However, this research used long bones (i.e. humeri, radii, ulnae, femora, tibiae, fibulae) and not foot bones (Mahakkanukrauh et al., 2011). Therefore, in order to determine how accurately the calcaneus and the talus estimate stature in comparison to other skeletal elements, the SEE range from the current research study is compared to the SEE ranges from previous research (Table 5.3). For consistency, the SEE range from the current research study (i.e. 3.06 to 7.36 ) has been rounded to the nearest tenth (i.e. 3.1 to 7.4 ). 
Table 5.3 Comparison of Standard Error of the Estimate (SEE) ranges for the current research study and previous studies by different authors.

\begin{tabular}{|c|c|c|c|}
\hline Investigator & Population & Skeletal element & SEE \\
\hline Jason and Taylor (1995) & American* & Vertebrae & $2.6-7.1$ \\
\hline Trotter and Gleser (1952a) & American* & $\begin{array}{l}\text { Humerus, radius, ulna, } \\
\text { femur, tibia, fibula }\end{array}$ & $3.0-5.1$ \\
\hline Current research study & Thai & Calcaneus, talus & $3.1-7.4$ \\
\hline Torimitsu et al. (2017) & Japanese & Clavicle & $3.4-4.9$ \\
\hline Shintaku and Furuya (1990) & Japanese & Phalanges & $3.6-4.3$ \\
\hline Torimitsu et al. (2015) & Japanese & Scapula & $3.8-6.3$ \\
\hline Bidmos and Asala (2005) & $\begin{array}{l}\text { Black South } \\
\text { African }\end{array}$ & Calcaneus & $4.0-5.9$ \\
\hline Byers et al. (1989) & American* & Metatarsals & $4.0-7.6$ \\
\hline Menezes $(2009,2011)$ & South Indian & Sternum & $4.1-5.6$ \\
\hline Bidmos (2006) & $\begin{array}{l}\text { White South } \\
\text { African }\end{array}$ & Calcaneus & $4.2-5.5$ \\
\hline Holland (1995) & Black American & Talus & $4.7-5.5$ \\
\hline Mahakkanukrauh et al. (2011) & Thai & $\begin{array}{l}\text { Humerus, radius, ulna, } \\
\text { femur, tibia, fibula }\end{array}$ & $4.9-6.1$ \\
\hline Meadows and Jantz (1992) & White American & Metacarpals & $5.1-5.7$ \\
\hline Zhang et al. (2016) & Chinese & Calcaneus & $5.1-10.5$ \\
\hline Torimitsu et al. (2016) & Japanese & Skull & $5.4-9.1$ \\
\hline Simmons et al. (1990) & American* & Fragmentary femur & $5.5-7.2$ \\
\hline Torimitsu et al. (2014) & Japanese & Sacrum & $5.8-8.3$ \\
\hline
\end{tabular}

*White American and Black American populations (combined ancestral sample)

A comparison of the SEE range from the current research study and the SEE range from the study by Mahakkanukrauh et al. (2011), suggests that in a contemporary 
Thai population, the calcaneus and the talus are more accurate when compared to intact long bones (i.e. intact humeri, radii, ulnae, femora, tibiae, fibulae). This seems to indicate that when the calcaneus and the talus are recovered in a forensic context, these bones should be used for the estimation of stature instead intact humeri, radii, ulnae, femora, tibiae, fibulae, because these bones may provide a more accurate estimation of stature in a contemporary Thai population. However, if the SEE value from the female simple regression equation using the MAXL measurement variable (i.e. 3.06, Table 4.9) is excluded, the SEE range from the current research study becomes 5.95 to 7.36. This indicates that overall, long bones provide a more accurate estimate of stature than the calcaneus and the talus in the contemporary Thai population. Therefore, if available in a forensic context, intact humeri, radii, ulnae, femora, tibiae, and fibulae should be used for stature estimation.

Overall, the SEE range from the current research study (3.06 to 7.36) suggests that the calcaneus and the talus may be less accurate when compared to the vertebral column (Jason and Taylor, 1995) and intact long bones (i.e. humeri, radii, ulnae, femora, tibiae, fibulae) (Trotter and Gleser, 1952a). Furthermore, the SEE ranges from studies by Shintaku and Furuya (1990) and Torimitsu and colleagues (2015) are, overall, lower than the SEE range from the current research study. This suggests that the calcaneus and the talus may also be less accurate when compared to the clavicle (Torimitsu et al., 2015) and the phalanges (Shintaku and Furuya, 1990). However, as regression equations for the estimation of stature are population-specific, the SEE ranges are only accurate for stature estimation in the population from which they were derived (i.e. White American, Black American, and Japanese populations; Jason and Taylor, 1995; Shintaku and Furuya, 
1990; Torimitsu et al., 2015; Trotter and Gleser, 1952a). Future studies should investigate the accuracy of these skeletal elements, particularly the vertebrae, for stature estimation in a contemporary Thai population.

While the results of the current research study demonstrate that the calcaneus and the talus may be accurate predictors of stature in a Thai population, previous studies have found contrasting results in other populations. For example, Zhang and colleagues (2016) found a high SEE range (i.e. SEE values from 5.05 to 10.5) in their study of stature estimation from the calcaneus in a Han Chinese population. Based on these results, Zhang and colleagues (2016) concluded that the calcaneus is not an accurate predictor of stature in the Han Chinese population. This study further demonstrates the necessity for population-specific regression formulae for stature estimation. However, the data utilized in the study by Zhang et al. (2016) were obtained from computed radiography (CR) technology, and thus, the results may not be as accurate as those obtained directly from bone (as in the current research study).

Studies have shown that projection, landmark identification, and measurement errors may occur using radiographic imaging technologies (Guenoun et al., 2012; Lagravère et al., 2010). Radiographic projection errors may be the result of magnification or distortion. Magnification occurs because the x-ray beams originate from a source that is not parallel to all points of the object (i.e. the bone) being examined. Distortion may occur because of unequal magnifications between different planes; skeletal landmarks may be affected by distortion because of their locations at different depth fields. Landmark identification error is influenced by factors such as quality of the radiographic image, which may in turn be influenced by the density and sharpness of images, 
superimposition of bone and other soft tissues, or differences in specimen orientation/positioning (Berg et al., 2007; Guenoun et al., 2012; Lagravère et al., 2010; Major et al., 1994; Spoor et al., 2000). These factors may influence the accuracy and reliability of measurements and result in measurement error. These factors may explain why Zhang and colleagues (2016) received such high SEE values for the calcaneus.

\subsection{Statistical Population Comparison}

The current research study has identified the need for population-specific regression formulae for the estimation of stature in unknown Thai skeletal remains. The third objective of this research was to compare the results from the contemporary Thai population to other populations. The contemporary Thai descriptive statistics were compared to the descriptive statistics of: Black South African (Bidmos and Asala, 2005), White South African (Bidmos, 2006), and Black American (Holland, 1995) populations.

\subsubsection{Comparison of contemporary Thai and Black South African (Bidmos and Asala,} 2005) descriptive statistics

Bidmos and Asala (2005) found that the calcaneus could be used to accurately estimate stature in a Black South African population. The descriptive statistics from the Black South African (Bidmos and Asala, 2005) population and the contemporary Thai population were compared (Table 4.15). Overall, the mean values of the calcaneal measurement variables, for both males and females, were larger in the contemporary Thai population than in the Black South African (Bidmos and Asala, 2005) population, with the exception of the male and female MAXH, MIDB, and DAFL measurement variables. 
The larger overall calcanei of the contemporary Thais when compared to the Black South Africans may be related to differences in their socioeconomic status or psychosocial stress.

During the South African apartheid (1948-1994), individuals were identified as Black (predominately of Bantu ancestry), White (of European ancestry), or Coloured (a heterogenous group comprised of individuals of Khoesan, Bantu, European, South Indian, and/or East Indian ancestry). There was a strong association between 'race' classification and socioeconomic status, which has continued during the country's transition to democracy (1994 to present day) (Christopher, 2001; Das-Munshi et al., 2016; Pfeiffer et al., 2016). The apartheid regime (1948-1994) led to a 'racially' segregated society in which Black South Africans were at a socioeconomic disadvantage to Whites and Coloureds (Das-Munshi et al., 2016). Overall, Black South Africans have experienced dietary deficiencies, inadequate state-subsidized health services, unemployment, inadequate, and often crowded accommodation/shelter, under-education, and general poverty (De Beer, 1984; Kark, 1974; Turton and Chalmers, 1990). Overall, lower socioeconomic status, marked by factors such as nutritional deficiencies, higher incidence of disease, and inadequate access to medical care, is correlated with decreased skeletal growth and development (Inwood and Roberts, 2010). These factors may have had a negative impact on the health and growth of Black South Africans and, thus, may be responsible for the smaller calcaneal dimensions observed in the Black South African (Bidmos and Asala, 2005) population.

Dietary information for Black South Africans during the apartheid era is limited, although it has been suggested that approximately $30 \%$ (and up to $75 \%$ in rural areas) of 
all Black South African children were malnourished (World Health Organization, 2005). Stupar (2007) states that, traditionally, the rural Black South African diet was not diverse or nutrient dense; staples of the Black South African diet included maize, vegetables, such as sweet potato or cabbage, and few meat products. Overall, studies have suggested that the nutritional status of Black South Africans was poor, with inadequate intake of calcium, vitamin D, and protein (Karaand and Myburgh, 1992; Naude et al., 2012, Pfeiffer et al., 2016; Stupar, 2007). Studies have shown that inadequate intake of calcium and vitamin D may compromise bone (specifically calcaneal) growth and development (Babaroutsi et al., 2005; Nathena et al., 2017; Naude et al., 2012; Stini, 1969). Furthermore, studies have shown that excessive alcohol use is correlated with calcium and vitamin D deficiencies. Excessive alcohol consumption has been known to negatively affect bone health by disturbing osteoblast activity and decreasing bone mass and strength (Naude et al., 2012). During the apartheid era, alcohol abuse was a common issue in many impoverished regions, and in many Black South African townships, very heavy alcohol use is still reported today (Kalichman et al., 2007, 2008; Pfeiffer et al., 2016; Schneider et al., 2007; Suliman et al., 2010). Poor nutrition in combination with heavy alcohol use may have compromised bone growth and skeletal development in the Black South African (Bidmos and Asala, 2005) population. Therefore, this may be the reason for the smaller calcaneal measurements in the Black South African population when compared to the Thai population.

The prevalence of alcohol use is relatively low in Thailand (especially in women), with consumption rates of only $21.6 \%$ to $22.22 \%$ among those aged 15 to 24 years (The National Statistics Office, 2008). The overall low prevalence of alcohol use and abuse 
among the general Thai population is likely the result of religious affiliations.

Approximately 95\% of the population in Thailand declare themselves Buddhist, which is the state religion in Thailand; Buddhism has a significant influence on Thai lifestyle and culture (Assanangkornchai et al., 2000, 2002; Limaye et al., 2013). Buddhists are expected to conform to certain moral injunctions known as the Five Precepts (Silas Ha), one of which is "the avoidance of distilled and fermented intoxicants causing carelessness" (Assanangkornchai et al., 2002: 193.) As a result, the Thai population shows enhanced bone growth and skeletal development when compared to the Black South African (Bidmos and Asala, 2005) population, which may explain the smaller calcaneal dimensions in the Black South African (Bidmos and Asala, 2005) population.

Differences in calcaneal dimensions between the Black South African (Bidmos and Asala, 2005) population and the contemporary Thai population may also be the result of socioeconomic differences related to living conditions, exposure to infectious disease and access to medical care (Dearden et al., 2017; Doak et al., 2002; Hong et al., 2006; Inwood and Roberts, 2010; Magnani et al., 1993; Merchant et al., 2003; Pongou et al., 2006).

Black South Africans, in the apartheid and post-apartheid eras, lived in often unsanitary and crowded conditions, which may have increased exposure to toxins and pollutants and thus increased their risk of disease (Dearden et al., 2017; Doak et al., 2002; Hong et al., 2006; Inwood and Roberts, 2010; Magnani et al., 1993; Merchant et al., 2003; Pongou et al., 2006). Turok and Borel-Saladin (2016) describe the conditions of squatter settlements inhabited by Black South Africans, citing that shacks of one to two rooms were often built by residents from materials such as wood or pieces of zinc or tin, 
and had no outdoor toilets or indoor running water. This type of accommodation is typical of many of the larger squatter settlements and townships South Africa (De Beer, 1984; Kark, 1974; Turok and Borel-Saladin, 2015; Turton and Chalmers, 1990). Studies have shown that individuals in households with a lack of sewage connection and indoor water connection were more likely to contract infectious diseases such as diarrhea, polio, or cholera, and parasites, and thus suffer growth and development deficits (Checkley et al., 2004; Haque, 2007; Hathi et al., 2017). Disease exposure and incidence can retard growth by diverting energy to fighting disease or by causing inadequate absorption of the nutrients consumed (Dearden et al., 2017; Doak et al., 2002; Hong et al., 2006; Inwood and Roberts, 2010; Magnani et al., 1993; Merchant et al., 2003; Pongou et al., 2006).

The incidence of such infectious diseases may be enhanced by high population density, facilitated by crowded living conditions, which provides more opportunities for disease transmission (Haque, 2007; Hathi et al., 2017). Overcrowding was common in many Black South African communities in the apartheid and post-apartheid eras, with an average of 17 people per household (De Beer, 1984; Kark, 1974; Turton and Chalmers, 1990).

Disease incidence may also be exacerbated by inadequate access to medical care. Access to health care has been limited for Black South Africans (De Beer, 1984; Kark, 1974; Turton and Chalmers, 1990). According to the American Association for the Advancement of Science and Physicians for Human Rights Organization (1998), the South African health care system has not only limited access to health care for Black South Africans, but facilitated an environment in which refusal of emergency treatment, denial or limitation of access of ongoing medical care, and mistreatment of the ill occurs. 
Overall, the inadequate living conditions, exposure to disease, and lack of access to proper medical care for Black South Africans may explain their smaller calcaneal dimensions when compared to the Thai population.

Research has also demonstrated that psychosocial stress during life may cause a decline in growth rates (Gilmour and Skuse, 1999; Peckmann et al., 2017; Ranabir and Reetu, 2011; Talbot et al., 1947; Turton and Chalmers, 1990) and therefore may be related to the calcaneal size differences between the Black South African (Bidmos and Asala, 2005) and Thai populations. Psychosocial stress is defined as the relationship between a person and the environment that is appraised by the person as taxing or exceeding their resources and endangering their well being (Lazarus and Folkman, 1984:19). In response to stress, hormone levels change; reactions to stress are associated with defects in the secretion of hormones such as glucocorticoids, catecholamines, growth hormone and prolactin (Ranabir and Reetu, 2011).

Turton and Chalmers (1990) state that discrimination may act as a stimulant to psychosocial stress, and studies have shown that the systemic 'racial' discrimination experienced by Black South Africans during the apartheid and post-apartheid eras have contributed to psychosocial distress among Black South Africans (Das-Munshi et al., 2016; Turton and Chalmers, 1990). Black South Africans, during the apartheid and postapartheid eras, were made to carry 'race' classification documentation and endured prejudicial practices such as the banning of 'inter-racial' marriage, residential segregation, forced removal from White-only residential areas, and required to use separate railway carriages and separate entrances at stations, post offices, and other business establishments (Seekings, 2008; Turton and Chalmers, 1990). Such 
discrimination may have contributed to psychosocial stress among Black South Africans, and thus negatively impacted hormone production causing stunted bone growth in the contemporary Black South African population. Therefore, psychosocial stresses may be responsible for the smaller calcaneal measurements illustrated by the Black South African (Bidmos and Asala, 2005) population when compared to the Thai population.

\subsubsection{Comparison of contemporary Thai and White South African (Bidmos, 2006) descriptive statistics}

Bidmos (2006) found that the calcaneus could also accurately estimate stature in a White South African population. The descriptive statistics from the White South African (Bidmos, 2006) population and the contemporary Thai population were compared (Table 4.16). Overall, the mean values of the calcaneal measurement variables, for both males and females, were smaller in the contemporary Thai population than in the White South African (Bidmos, 2006) population, with the exception of the male and female CFH, LAL, and DAFB measurement variables, and the female MINB measurement variable. The larger calcaneal dimensions of the White South African population may be the result of differences in genotype, socioeconomic factors, and occupational stressors.

Studies have demonstrated the effect of genotype and genetic factors on skeletal growth; twin and family studies have suggested that as much as $90 \%$ of variation in human growth is genetically determined (Knight et al., 2005; Lettre et al., 2008; Macgregor et al., 2006; Perola et al., 2007; Preece, 1996; Rappold et al., 2007;

Silventoinen et al., 2000, 2001; Weedon et al., 2007, 2008; Zhao et al., 2010). There have been many genome wide association (GWA) studies that have identified a number of 
genes and genetic loci influencing skeletal growth (Carty et al., 2012; Estrada et al., 2009; Johansson et al., 2009; Lettre et al., 2008; Liu et al., 2010; Sanna et al., 2008; Soranzo et al., 2009; Tönjes et al., 2009; Weedon et al., 2007, 2008). Although, very few studies have been conducted in Asian populations. One study, however, by Lei and colleagues (2009) found that the genomic region containing the ZNP510 and ZNP782 genes is an "ethnic-specific" locus associated with bone growth in Asian individuals. Similarly, Hao and colleagues (2013) identified three novel loci for bone growth, which mapped in or near ZNF638 (rs12612930), MAML2 (rs11021504), and C18orf12 (rs11082671). These loci were found in a Han Chinese population but not identified individuals of other ancestral backgrounds, indicating that these loci may be specific to Asian individuals (Hao et al., 2013).

Contemporary Thai populations show genetic contributions from South Asians, Southeast Asians, Northwest Asians, and Polynesians (Peckmann et al., 2017; Xing et al., 2010). The genetic composition of the White South African (Bidmos, 2006) population group includes contributions from migrants of the Netherlands, United Kingdom, France, Germany, Portugal and other European groups, as well as admixture with local (i.e. South African) groups (Bidmos, 2006; Bidmos and Asala, 2003; Pfeiffer et al., 2016; Steyn and Işcan, 1997). As the genetic composition of the contemporary Thai population and the White South African (Bidmos, 2006) population is different, and given that previous authors have identified many Asian-specific loci associated with skeletal growth and development, genotype may be the reason for the mean calcaneal differences observed between the contemporary Thai and the White South African (Bidmos, 2006) population. 
Calcaneal growth and development may also be affected by socioeconomic conditions (Bogin et al., 2002; Dangour, 2001; Inwood and Roberts, 2010; Nyati et al., 2006). The individuals utilized for the current research study were from Chiang Mai and adjacent villages in northern Thailand and were of middle and low socioeconomic status (King et al., 1998; Traithepchanapai et al., 2016). Poverty in Thailand exists as a mainly rural phenomenon; as of 2014 , over $80 \%$ of the country's poor live in rural areas and pockets of poverty remain in the North and Northeast regions (World Bank, 2017). Many of the White South African individuals in the Bidmos (2006) study lived during the apartheid era (1948-1994) (Steyn and Işcan, 1997). During this time, White South Africans experienced a higher socioeconomic status when compared to other groups in the country, including better living conditions and limited overall poverty (Kon and Lackan, 2008; Turton and Chalmers, 1990; van der Berg et al., 2006). Therefore, the differences in living conditions may be responsible for the larger calcaneal dimensions observed in the White South African (Bidmos, 2006) population when compared to the Thai population.

Nutritional deficiencies may comprise bone growth and development (Babaroutsi et al., 2005; Nathena et al., 2017; Naude et al., 2012; Stini, 1969). Food intake and proper nutrition may be compromised when food is in short supply (in instances such as war or famine), or because the price of food groups important to growth, such as protein, increases above what some individuals are able to pay (Bogin, 1999; Inwood and Roberts, 2010). The Food and Agricultural Organization of the United Nations (2012) has reported that there is a high level of undernourishment in Thailand. In particular, studies have shown that the habitual diet among rural Northern Thai individuals does not meet 
recommended calcium, protein, zinc, vitamin A or vitamin C intake levels, thus resulting in stunted growth (Pongchaiyakul et al., 2008; Rojroongwasinkul et al., 2013). In a study by Pongchaiyakul and colleagues (2008), it was observed that $67 \%$ of contemporary Thai men and $87 \%$ of contemporary Thai women had a dietary calcium intake of less than half the recommended level ( $<400$ milligrams per day). As calcium is the dominant mineral in bone, the authors' note that such deficiencies may result in an acceleration of bone loss, increased risk of osteoporosis, increased risk of bone fracture, and overall impaired bone growth and development (Pongchaiyakul et al., 2008).

In contrast, the White South African population experienced adequate nutrition with minimal nutritional deficiencies or diet-related growth impairments (Kon and Lackan, 2008; Turton and Chalmers, 1990; van der Berg et al., 2006). Overall, studies have shown that White South Africans during the apartheid and post-apartheid eras were food secure (i.e. did not experience hunger) and experienced high dietary diversity (Kon and Lackan, 2008; Stupar, 2007; Turton and Chalmers, 1990; van der Berg et al., 2006). Dietary diversity can be used as a proxy measure of the nutritional quality of a population's diet (Kennedy et al., 2009). The high dietary diversity of the White South African population thus indicates adequate intake of essential nutrients. Therefore, the nutritional deficiencies and inadequate calcium intake in contemporary Thai populations may have caused impaired bone growth and development, which may explain the larger calcaneal dimensions observed in the White South African (Bidmos, 2006) population when compared to the Thai population.

Differences in the mean calcaneal dimensions between the White South African (Bidmos, 2006) population and the contemporary Thai population may also be the result 
of socioeconomic differences related to exposure to toxins and/or pollutants, risk of disease, and access to medical care (Dearden et al., 2017; Doak et al., 2002; Hong et al., 2006; Inwood and Roberts, 2010; Magnani et al., 1993; Merchant et al., 2003; Pongou et al., 2006). During apartheid, areas with higher living standards were designated "Whiteonly" (Turton and Chalmers, 1990). The high socioeconomic status of White South Africans (Bidmos, 2006) means that this group benefitted from more affluent living conditions, proper sanitation, clean water access, and low population density. These conditions may also reduce exposure to toxins or pollutants and reduce the risk of disease in the White South African (Bidmos, 2006) population (Dearden et al., 2017; Doak et al., 2002; Hong et al., 2006; Inwood and Roberts, 2010; Magnani et al., 1993; Merchant et al., 2003; Pongou et al., 2006). Therefore, greater socioeconomic conditions may be responsible for the larger calcaneal dimensions observed in the White South African (Bidmos, 2006) population when compared to the Thai population.

Finally, increases in the intensity or the volume of physical activity without increasing caloric intake may decrease the calories available for bone growth, thus retarding bone growth and influencing final bone size (Steckel, 2008). The individuals represented in the contemporary Thai population are from the northern region of Thailand, and area in which rice farming is popular (Arunrat and Pumijumnong, 2015; Isvilanonda and Bunyasiri, 2009). Rice farming involves the navigation of uneven terrain and hard manual labour, which requires high caloric expenditure. The diet of the contemporary Thai population has been shown to be nutritionally deficient, resulting in high levels of undernourishment in Thailand (Pongchaiyakul et al., 2008;

Rojroongwasinkul et al., 2013). The high intensity and volume of physical activity (i.e. 
the high caloric expenditure) experienced by the contemporary Thai population, in combination with the compromised caloric intake of contemporary Thais, would have decreased calories available for growth and may have contributed to deficiencies in bone growth in the contemporary Thai population.

The White South African (Bidmos, 2006) population is more urban than the contemporary Thai population, with higher levels of education and as a result, has enhanced economic and urban labour market opportunities (Kon and Lackan, 2008; Turton and Chalmers, 1990; van der Berg et al., 2006). Many of the White South African individuals in the Bidmos (2006) study lived during the apartheid era (1948-1994) (Steyn and Işcan, 1997). Employment data demonstrate that during this time, the majority of White South Africans during the apartheid were employed "skilled" occupations (i.e. managerial, professional, and technician positions), specifically in positions that are considered "white collar", such as administration, management, executive, and clerical positions (Mariotti, 2012; van Wyk, 1968). As a result of these labour activities, the White South African (Bidmos, 2006) population likely experienced low caloric expenditure, as the physical intensity of daily activities would have been low. Furthermore, as discussed earlier in this chapter, the White South African (Bidmos, 2006) population experienced adequate nutrition with minimal nutritional deficiencies and substantial caloric intake (Kon and Lackan, 2008; Turton and Chalmers, 1990; van der Berg et al., 2006). The low caloric expenditure and high caloric intake experienced by the White South African (Bidmos, 2006) population would have been conducive to bone growth. This may explain the larger calcaneal dimensions observed in the White South African (Bidmos, 2006) population in comparison to the contemporary Thai population. 
5.5.3 Comparison of contemporary Thai and Black American (Holland, 1995) descriptive statistics

Holland (1995) found that the talus may be used to accurately estimate stature in a Black American population. The descriptive statistics from the Black American population (Holland, 1995) and the contemporary Thai population were compared (Table 4.17). Overall, the mean values of the one talar measurement variable, for both males and females, were larger in the Black American (Holland, 1995) population than the contemporary Thai population.

The individuals utilized for the current research study were from Chiang Mai and adjacent villages in northern Thailand and were of middle and low socioeconomic status (King et al., 1998; Traithepchanapai et al., 2016). Holland (1995) utilized Black American individuals from the Hamann-Todd Collection at the Cleveland Museum of Natural History. These individuals are representative of a mixed temporal population, i.e. an historic Black American population and a modern early $20^{\text {th }}$ century Black American population. The population shows dates of birth ranging from 1823 to 1924 and dates of death ranging from 1908 to 1939 (personal communication, LM Jellema, Collections Manager, Cleveland Museum of Natural History, January 2017). As this population is representative of an enslaved African and early historic Black American population, this population was also of low socioeconomic status (Blonigen, 2004; Carson, 2010; Spradley, 2006). Overall, both the Black American (Holland, 1995) population and the contemporary Thai population were of depressed backgrounds, with poor nutrition, poor sanitation and high exposure to toxins and/or pollutants, increased risk of disease, and inadequate access to medical care. Therefore, differences in socioeconomic status cannot 
account for differences in talar dimensions observed between the Black American (Holland, 1995) population and the contemporary Thai population.

Furthermore, the Thai individuals are from the Northern region of Thailand, an area in which rice farming is popular (Arunrat and Pumijumnong, 2015; Isvilanonda and Bunyasiri, 2009). Rice farming involves the navigation of uneven terrain and hard manual labour. Specifically, rice farming requires individuals to bear large amounts of weight on the head, shoulders, or back during seed transplantation and harvesting (Barton, 1960). The individuals utilized by Holland (1995) (i.e. enslaved Africans and early historic Black Americans) were mainly unskilled workers involved in domestic or agricultural positions, which also required hard manual labour (Blonigen, 2004; Carson, 2010). Black Americans working on plantations, for example, were responsible for tasks such as chopping trees, repairing cabins, tending gardens, and harvesting, which may include specific tasks such as vigorous hoeing, weed pulling, thrashing, and sifting (Blonigen, 2004). As both populations were engaged in similar occupational activites, which required hard manual labour and intense physical activity, therefore differences in occupational activities cannot account for the differences in talar dimensions observed between the Black American (Holland, 1995) population and the contemporary Thai population.

Overall, the larger tali of Black Americans (Holland, 1995), in comparison to the contemporary Thai population, may be the result of differences in genotype. Studies have demonstrated the effect of genotype and genetic factors on skeletal growth (Knight et al., 2005; Lettre, 2008; Macgregor et al., 2006; Perola et al., 2007; Preece, 1996; Rappold et al., 2007; Silventoinen et al., 2000, 2001; Weedon et al., 2007, 2008; Zhao et al., 2010). 
As well, the published literature has demonstrated the existence of genetic variants associated with bone growth, which are specific to Asian individuals (Hao et al., 2013; Lei et al., 2009). For example, Hao and colleagues (2013) identified three novel loci that mapped in or near ZNF638 (rs12612930), MAML2 (rs11021504), and C18orf12 (rs11082671), which were found in a Han Chinese population but not identified individuals of other ancestral backgrounds. These results demonstrate that there are differences in the genetic variants associated with bone growth between populations of different ancestral backgrounds.

Contemporary Thai populations show genetic contributions from South Asians, Southeast Asians, Northwest Asians, and Polynesians (Peckmann et al., 2017; Xing et al., 2010). As discussed previously in this chapter, Holland (1995) utilized Black American individuals representative of a mixed temporal population, i.e. an historic Black American population and a modern early $20^{\text {th }}$ century Black American population. The genotype of this Black American (Holland, 1995) population was influenced by varying levels of African and White European ancestry (Spradley, 2006). As the genetic composition of the contemporary Thai population and the Black American (Holland, 1995) population is different, and given that previous authors have identified many Asian-specific loci associated with skeletal growth and development, genotype may be the reason for the mean talar differences observed between the contemporary Thai and the Black American (Holland, 1995) populations. 


\section{CHAPTER 6: CONCLUSIONS}

Within the current body of literature for stature estimation, Thai populations are underrepresented; prior to the current project, there has only been one study completed on stature estimation in a contemporary Thai population (Mahakkanukrauh et al., 2011). In Thailand, the 2004 tsunami disaster highlighted the lack of forensic anthropology expertise in the country and the lack of population-specific methods in the region (Traithepchanapai et al., 2016). The current research study addresses this need, and focuses on the calcaneus and the talus to establish an accurate method of living stature estimation for a contemporary Thai population. The objectives of this research are to:

1. investigate whether regression equations for the calcaneus, which were developed on a Black South African population (Bidmos and Asala, 2005), and regression equations for the talus, which were developed on a Black American population (Holland, 1995), can accurately predict the living stature of individuals from a contemporary Thai population.

2. develop population-specific simple regression and multiple regression equations for the calcaneus and the talus of the contemporary Thai population should the Black South African (Bidmos and Asala, 2005) and Black American (Holland, 1995) equations not accurately predict the living stature of the Thai individuals.

3. compare the results from the contemporary Thai population to other populations.

This study examined the calcaneus and the talus as they are small, compact bones with dense trabeculae and small surface area, and are often encased in socks and/or shoes, which serve as protection for the feet (Bidmos and Asala, 2005; Peckmann et al., 2017; 
Pickering, 1986; Saul and Saul, 2005). These factors result in resiliency to taphonomic stresses, leading to enhanced preservation for stature estimation in forensic cases.

In the current research study, the paired calcaneus and talus of 232 adult skeletonized individuals (115 males, 117 females) of Thai ancestry were examined for their potential in estimating stature. In total, 464 individual bones $\left(\mathrm{N}^{\text {total bones }}=464 ; \mathrm{N}^{\text {calcaneus }}=232\right.$, $\mathrm{N}^{\text {talus }}=232$ ) were examined from the Chiang Mai skeletal collection, which represents a contemporary population. Only calcanei and tali of skeletally adult individuals (between 19 years and 96 years of age) were studied to ensure that the calcaneus and the talus are skeletally mature and have reached their maximum size. Individuals were excluded from this study if both the calcaneus and the talus (from an individual pair) were not present for analysis, or if the bones exhibited any form of taphonomic damage, post mortem trauma, or extreme pathologic remodeling of the morphometric characteristics which would inhibit the collection of accurate measurements. The vast majority of skeletons in the Chiang Mai skeletal collection exhibited some osteoarthritic bone remodeling. Therefore, calcanei and tali exhibiting mild osteoarthritis, in which measured morphometric traits of the calcaneus and the talus were not affected, were included in this study because excluding these individuals would bias the sample from accurately representing the Thai population as a whole.

Following the protocol of Bidmos and Asala (2005), nine measurements (i.e. “measurement variables") were collected from the calcaneus: Maximum Length (MAXL), Maximum Height (MAXH), Cuboidal Facet Height (CFH), Body Height (BH), Minimum Breadth (MINB), Load Arm Length (LAL), Middle Breadth (MIDB), Dorsal Articular Facet Breadth (DAFB), and Dorsal Articular Facet Length (DAFL). Following 
the protocol of Holland (1995), one measurement variable was collected for the talus: Maximum Length of the Talus (MTAL). A total of ten measurement variables were collected for each individual, i.e. nine measurements of the calcaneus and one measurement of the talus.

Sexual dimorphism was statistically analyzed using two-sample t-tests. All calcaneal and talar measurement variables were found to be sexually dimorphic $(p<0.005)$, which indicates that these measurement variables have good predictive value for estimating living stature for both contemporary Thai males and contemporary Thai females. For the calcaneus, the MAXL measurement variable exhibited the greatest dimorphic variation between males and females. For the talus, the MTAL measurement variable exhibited the greatest dimorphic variation between males and females. The variations of sexually dimorphic expression exhibited in the MAXL and MTAL measurement variables, between populations, may be due to differences in genetics, socioeconomic status, and habitual or occupational behaviours.

Intra-observer and inter-observer error were statistically analyzed using paired t-tests and concordance correlation of reproducibility method (Lin, 1989). Intra-observer error showed no statistically significant difference between the original sample and the remeasured sub-sample $(p>0.005)$. However, when statistically analyzed using Lin's (1989) concordance correlation, four of the nine measurement variables (male DAFB, female $\mathrm{CFH}, \mathrm{BH}, \mathrm{MINB}$, and DAFB) did not fall within the internationally accepted standard range of 0.90 to 0.99 (Cameron, 1984; Lin, 1989). Inter-observer error also showed no statistically significant difference between the original sample and the remeasured sub-sample $(p>0.005)$. However, when statistically analyzed using Lin's 
(1989) concordance correlation, three of the nine measurement variables (male DAFB, female $\mathrm{BH}$ and MINB) did not fall within the internationally accepted standard range of 0.90 to 0.99 (Cameron, 1984; Lin, 1989). This indicates that these measurements may not be accurately repeated and as such caution must be taken when including these variables in future studies (Berg et al., 2007). Given the results of the current research study, the current author suggests that forensic practitioners utilize the male DAFB measurement variable and the female $\mathrm{CFH}, \mathrm{MINB}, \mathrm{DAFB}$, and $\mathrm{BH}$ measurement variables during anthropological assessment only when other, more reliable (i.e. those with more accurate repeatability) measurement variables are unavailable for examination, in order to best satisfy the Mohan and Daubert admissibility criteria.

To determine whether published regression equations for the calcaneus can accurately predict the living stature of individuals from a contemporary Thai population, each of the simple and multiple regression equations for the calcaneus and for the talus, that were generated from a Black South African (Bidmos and Asala, 2005) and Black American (Holland, 1995) population, respectively, were tested on the Thai sample data. Overall, correlation coefficient values ( $r$-values) decreased when Black South African (Bidmos and Asala, 2005) and Black American (Holland, 1995) regression equations were applied to the contemporary Thai population. The lowered accuracy rates for the Bidmos and Asala (2005) calcaneal regression formulae and the Holland (1995) talar regression formulae when applied to the contemporary Thai population suggest that regression formulae are population-specific and that there is variation in skeletal features of the calcaneus and the talus between populations. 
To date, no study has used the calcaneus and/or the talus for the estimation of stature in a contemporary Thai population. The calcaneus, the talus, and a combination of the two foot bones, were used to create simple and multiple regression equations for living stature estimation in the contemporary Thai population. A total of 18 simple regression equations were generated from the calcaneus $\left(\mathrm{N}^{\text {total calcaneal simple regression equations }}=18 ; \mathrm{N}^{\text {male }}\right.$ calcaneal simple regression equations $=9, \mathrm{~N}^{\text {female calcaneal simple regression equations }}=9$ ), a total of two simple regression equations were generated from the talus $\left(\mathrm{N}^{\text {total talar simple regression equations }}=2 ; \mathrm{N}^{\text {male }}\right.$ talar simple regression equations $=1, \mathrm{~N}^{\text {female talar simple regression equations }}=1$ ), and a total of 10 multiple regression equations were generated from a combination of calcaneal and talar measurement variables $\left(\mathrm{N}^{\text {total multiple regression equations }}=10 ; \mathrm{N}^{\text {male multiple regression equations }}=5\right.$, $\mathrm{N}^{\text {female multiple regression equations }}=5$ ).

The accuracy of stature estimation from bones using regression equations is measured by the Standard Error of the Estimate (SEE); the lower the SEE as presented by a regression equation, the more accurate is the estimate of stature. For contemporary Thai males, the lowest SEE is observed when utilizing all ten measurement variables of the calcaneus and the talus (Function 1; SEE = 5.70). This suggests that all measurement variables of the calcaneus and the talus should be utilized when estimating stature for contemporary Thai males, in order to provide the most accurate results. For contemporary Thai females, the lowest SEE is observed when utilizing only the MAXL calcaneal measurement variable $(\mathrm{SEE}=3.06)$. This suggests that utilizing combinations of measurement variables, or including the talus in the analysis, does not increase the accuracy of stature estimation in a contemporary female Thai population. Therefore, 
when estimating stature for contemporary Thai females, only the MAXL measurement variable should be used in order to obtain the most accurate results.

A cross-validation study evaluated the accuracy of the simple and multiple regression equations for the estimation of stature by testing the predictive performance of each simple and multiple regression equation on an independent sample (Sample 2), which was not used to generate the simple and multiple regression equations. Following the protocol of Bidmos and Asala (2005) for the calcaneus and Holland (1995) for the talus, the accuracy of the estimation of living stature for each of the calcaneal and talar simple and multiple regression equations was assessed by calculating in what percent of cases, from Sample 2, did the Thai predicted living stature fall within 1 Standard Error of the Estimate (1SEE) and 2 Standard Errors of the Estimate (2SEE) of the Thai documented living stature. Using calcaneal simple regression equations, the estimated living stature fell within 1 SEE in $50 \%$ to $67 \%$ of cases for contemporary Thai males, while the accuracy was higher at 2SEE (87\% to 97\%). For Thai females, living stature estimates fell within 1SEE in $53 \%$ to $70 \%$ of cases and within 2SEE in $90 \%$ to $97 \%$ of cases using calcaneal simple regression equations. Using talar simple regression equations, the estimated living stature fell within 1 SEE in $53 \%$ of cases and within 2SEE in $90 \%$ of cases for contemporary Thai males. For contemporary Thai females, the living stature estimations generated from the MTAL simple regression equation for the talus fell within 1 SEE in $70 \%$ of cases and within 2 SEE in $93 \%$ of cases. Using multiple regression equations, the predicted living stature fell within 1 SEE in $47 \%$ to $57 \%$ of cases for contemporary Thai males, while the accuracy was higher at 2SEE (83\% to 90\%). Contemporary Thai females however, showed a higher percentage accuracy as the 
predicted living stature fell within 1 SEE in $63 \%$ to $70 \%$ of cases and within 2 SEE in $87 \%$ to $93 \%$ of cases when utilizing multiple regression equations.

The contemporary Thai descriptive statistics were compared to the descriptive statistics of: Black South African (Bidmos and Asala, 2005), White South African (Bidmos, 2006) and Black American (Holland, 1995) populations. Statistical analyses indicated that that all measurement variables of the calcaneus show statistically significant differences between the contemporary Thai population and the Black South African population (Bidmos and Asala, 2005), except the male MAXL, MAXH, MINB and the female MAXL, MAXH, BH. Overall, the mean values of the calcaneal measurement variables, for both males and females, were larger in the contemporary Thai population than in the Black South African population (Bidmos and Asala, 2005) with the exception of the male and female MAXH, MIDB, and DAFL measurement variables. When compared to a White South African population (Bidmos, 2006), statistical analyses indicated that that all measurement variables of the calcaneus show statistically significant differences between the contemporary Thai population and the White South African population (Bidmos, 2006), except the male BH, MINB, LAL, and female BH, MINB, and MIDB measurement variables. Overall, the mean values of the calcaneal measurement variables, for both males and females, were smaller in the contemporary Thai population than in the White South African population (Bidmos, 2006), with the exception of the male and female CFH, LAL, and DAFB measurement variables, and the female MINB measurement variable. For the talus, statistical analyses indicated that all measurement variables of the talus showed a statistically significant difference between the contemporary Thai population and the Black American population (Holland, 1995). 
Overall, the mean values of the one talar measurement variable, for both males and females, were larger in the Black American population (Holland, 1995) than the contemporary Thai population. The variations in calcaneal and talar morphology between populations may be due to differences in socioeconomic status, psychosocial stress, genotype, or occupational stressors.

Overall, the results of the current research study demonstrate that regression equations for the calcaneus, which were developed on a Black South African population (Bidmos and Asala, 2005), and regression equations for the talus, which were developed on a Black American population (Holland, 1995), do not provide high enough accuracy rates for the estimation of stature in a contemporary Thai population. This finding, in addition to the comparison of descriptive statistics, further supports the notion that regression formulae for the estimation of stature are population-specific. The methods of stature estimation generated in the current research study should only be used for the Thai population and not be applied to other Southeast Asian populations.

Future research should continue to expand the data on available calcaneal and talar dimensions for the estimation of stature for contemporary Thais by collecting data across the country. The population-specific regression equations generated in the current research study would need to be tested using samples from different geographic regions in Thailand. It would be especially important to evaluate the regression equations generated in the current research study using an urban contemporary Thai population, in order to better examine the role of genetic, socioeconomic, and environmental factors on calcaneal and talar morphology. Furthermore, future studies should test the regression formulae generated in the current research study on geographically distinct populations of 
Thai ancestry, as well as other Southeast Asian populations; this would help determine if these data would accurately predict stature for other Southeast Asian populations.

Lastly, future studies should aim to generate regression formulae for the estimation of stature, in the Thai population, using other skeletal elements. To date, there has only been one study completed on stature estimation in a contemporary Thai population, which used long bones (i.e. humeri, radii, ulnae, femora, tibiae, fibulae) (Mahakkanukrauh et al., 2011). The creation of regression formulae from other skeletal elements will help to determine if there are other skeletal elements that may be more accurate for the estimation of stature in a contemporary Thai population. Given that climate change events such as flooding and natural disasters are predicted to increase in both frequency and severity in Thailand, resulting in a higher number of deaths, it is crucial that population-specific methods of victim identification be derived for a Thai population. Ultimately, the creation of new regression formulae for the estimation of stature will help forensic practitioners accurately identify the remains of contemporary Thais in mass disaster contexts, and also in cases of missing persons unrelated to mass disaster scenarios. 


\section{REFERENCES}

Abd-elaleem, S. A., M. Abd-elhameed, and A. A. Ewis

2012 Talus Measurements as a Diagnostic Tool for Sexual Dimorphism in Egyptian Population. Journal of Forensic and Legal Medicine 19(2): 70-76.

Adams, B. J.

2007 Forensic Anthropology. New York: Infobase Publishing.

Agarwal, A. K., P. Jeyasingh, S. C. Gupta, C. D. Gupta, and A. Sahai 1984 Peroneal Tubercle and Its Variations in the Indian Calcanei. Anatomischer Anzeiger 156(3): 241-244.

Agnihotri, A. K., S. Kachhwaha, K. Googoolye, and A. Allock 2011 Estimation of Stature from Cephalo-Facial Dimensions by Regression Analysis in Indo-Mauritian Population. Journal of Forensic and Legal Medicine 18(4): 167-172.

Ahmed, A, A., and S. Taha 2016 Cephalo-Facial Analysis to Estimate Stature in a Sudanese Population. Legal Medicine 20: 80-86.

Albanese, J.

2010 A Critical Review of the Methodology for the Study of Secular Change Using Skeletal Data. Ontario Archaeology Occassional Publication No.9.

Albanese, J., and S. R. Saunders 2006 Is it Possible to Escape Racial Typology in Forensic Identification? In Forensic Anthropology and Medicine: Complementary Sciences from Recovery to Cause of Death. Aurore Schmitt, Eugenia Cunha and Joao Pinheiro, eds. Pp. 281316. Totowa: Humana Press.

Allen, $\mathrm{T}$.

1994 The Invention of the White Race, vol.1. Haymarket Series. London: New York: Verso.

American Association for the Advancement of Science and Physicians for Human Rights 1998 Human Rights and Health: The Legacy of Apartheid. Accessed September 25, 2017.

http://shr.aaas.org/loa/contents.htm.

Ammann, P., and R. Rizzoli

2003 Bone Strength and Its Determinants. Osteoporosis International: A Journal Established as Result of Cooperation between the European Foundation for 
Osteoporosis and the National Osteoporosis Foundation of the USA 14 Suppl 3: S13-18.

Anderson, K. G.

2015 Father Absence, Childhood Stress, and Reproductive Maturation in South Africa. Human Nature 26(4): 401-425.

Anthes, R.A, R. W. Corell, G. Holland, J. W. Hurrell, M. C. MacCracken, and K. E. Trenberth 2006 Hurricanes and Global Warming - Potential Linkages and Consequences. Bulletin of The American Meteorological Society 87(5): 623-628.

Armelagos, G. J.

1998 Introduction: Sex, Gender and Health Status in Prehistoric and Contemporary Populations. In Sex and Gender in Paleopathological Perspective. A. L. Grauer and P. Stuart-Macadam, eds. Pp. 1-10. Cambridge: Cambridge University Press.

Arunrat, N., and N. Pumijumnong 2015 The Preliminary Study of Climate Change Impact on Rice Production and Economic in Thailand 11(15): 275-294.

Assanangkornchai, S., J. B. Saunders, and K. M. Conigrave 2000 Patterns of Drinking in Thai Men. Alcohol and Alcoholism 35(3): 263-269.

Assanangkornchai, S., K. M. Conigrave, and J. B. Saunders 2002 Religious Beliefs and Practice, and Alcohol Use in Thai Men. Alcohol and Alcoholism 37(2): 193-197.

Auerbach, B. M., and C. B. Ruff 2010 Stature Estimation Formulae for Indigenous North American Populations. American Journal of Physical Anthropology 141(2): 190-207.

Babaroutsi, E., F. Magkos, Y. Manios, and L. S. Sidossis 2005 Body Mass Index, Calcium Intake, and Physical Activity Affect Calcaneal Ultrasound in Healthy Greek Males in an Age-Dependent and Parameter-Specific Manner. Journal of Bone and Mineral Metabolism 23(2): 157-166.

Bailey, S. M., and S. M. Garn 1986 The Genetics of Maturation. In Human Growth. F. Falkner and J. M. Tanner, eds. 2nd edition. Pp. 169-195. New York: Plenum Press. 
Balvir, T. K., J. V. Deshpande, P. Badwaik, A. S., Rahule, A. Kasote, and M. S. M. Bashir 2012 Estimation of Stature from the Lenth of Clavicle in Vidarbha Region of Maharashtra. International Journal of Biological and Medical Research (3)3: 2254-2256.

Bamshad, M.

2005 Genetic Influences on Health: Does Race Matter? JAMA 294(8): 937-946.

Barnett, C. H.

1954 Squatting Facets on the European Talus. Journal of Anatomy 88(4): 509513.

Barrett, C. H., W. Cavallari, and P. W. Sciulli

2001 Estimation of Sex from the Talus in Prehistoric Native Americans. Collegium Antropologicum 25(1): 13-19.

Barth, F.

1998 Ethnic Groups and Boundaries: The Social Organization of Culture Difference. Prospect Heights, IL: Waveland Press.

Barton, T. F.

1960 Growing Rice in Thailand. Journal of Geography 59(4): 153-159.

Bau, A. M., A. Ernert, L. Schenk, S. Wiegand, P. Martus, A. Grüters, and H. Krude 2009 Is There a Further Acceleration in the Age at Onset of Menarche? A CrossSectional Study in 1840 School Children Focusing on Age and Bodyweight at the Onset of Menarche. European Journal of Endocrinology 160(1): 107-113.

Berenter, J. S., and F. D. Goldman 1989 Surgical Approach for Enlarged Peroneal Tubercles. Journal of the American Podiatric Medical Association 79(9): 451-454.

Berg, G. E., S. C. Ta'ala, E. J. Kontanis, and S. S. Leney 2007 Measuring the Intercondylar Shelf Angle Using Radiographs: Intra- and Inter-Observer Error Tests of Reliability. Journal of Forensic Sciences 52(5): $1020-1024$.

Bidmos, M. A.

2005 On the Non-Equivalence of Documented Cadaver Lengths to Living Stature Estimates Based on Fully's Method on Bones in the Raymond A. Dart Collection. Journal of Forensic Sciences 50(3): 501-6.

Bidmos, M. A. 2006 Adult Stature Reconstruction from the Calcaneus of South Africans of European Descent. Journal of Clinical Forensic Medicine 13(5): 247-252. 
Bidmos, M. A. 2008 Metatarsals in the Estimation of Stature in South Africans. Journal of Forensic and Legal Medicine 15(8): 505-509.

Bidmos, M. A., and M. R. Dayal 2003 Sex Determination from the Talus of South African Whites by Discriminant Function Analysis. The American Journal of Forensic Medicine and Pathology 24(4): 322-328.

Bidmos, M. A., and M. R. Dayal 2004 Further Evidence to Show Population Specificity of Discriminant Function Equations for Sex Determination Using the Talus of South African Blacks. Journal of Forensic Sciences 49(6): 1165-1170.

Bidmos, M. A, and S. Asala 2005 Calcaneal Measurement in Estimation of Stature of South African Blacks. American Journal of Physical Anthropology 126(3): 335-342.

Black, R. E., C. G. Victora, S. P. Walker, Z. A. Bhutta, P. Christian, M. de Onis, M. Ezzati, S. G. McGregor, J. Katz, R. Martorell, and R. Uauy 2013 Maternal and Child Undernutrition and Overweight in Low-Income and Middle-Income Countries. The Lancet 382(9890): 427-451.

Blau, S., and C. A. Briggs 2011 The Role of Forensic Anthropology in Disaster Victim Identification (DVI). Forensic Science International 205(1): 29-35.

Blonigen, B. 2004 A Re-Examination of the Slave Diet. Honours Thesis, College of St.Benedict/St.John's University.

Blumenbach, J. F, T. Bendyshe, K. F. H. Marx, P. Flourens, W. Rudolf, and J. D. Hunter 1865 The Anthropological Treatises of Johann Friedrich Blumenbach. London: Published for the Anthropological Society, by Longman, Green, Longman, Roberts, \& Green. http://archive.org/details/anthropologicalt00blumuoft, accessed February 25, 2017.

Boas, F.

1912 Changes in the Bodily Form of Descendants of Immigrants. American Anthropologist 14(3): 530-562.

Bogin, B., P. Smith, A. B. Orden, M. I. Varela Silva, and J. Loucky 2002 Rapid Change in Height and Body Proportions of Maya American Children. American Journal of Human Biology 14(6): 753-761. 
Bolin, B.

1986 The Greenhouse Effect, Climatic Change, and Ecosystems. SCOPE Report 29. Chichester (West Sussex); New York: Published on behalf of the Scientific Committee on the Problems of the Environment of the International Council of Scientific Unions by Wiley.

Bourguignon, J. P.

1988 Linear Growth as a Function of Age at Onset of Puberty and Sex Steroid Dosage: Therapeutic Implications. Endocrine Reviews 9(4): 467-488.

Boyd, D. C.

1996 Skeletal Correlates of Human Behavior in the Americas. Journal of Archaeological Method and Theory 3(3): 189-251.

Brace, C. L., and A. S. Ryan

1980 Sexual Dimorphism and Human Tooth Size Differences. Journal of Human Evolution 9(5): 417-435.

Brandt, E. T.

2009 Stature Wars: Which Stature Estimation Methods are Most Applicable to Modern Populations? Master's Thesis, Texas State University

Buikstra, J. E., and D. H. Ubelaker 1994 Standards for Data Collection from Human Skeletal Remains. Fayetteville, Ark.: Arkansas Archeological Survey.

Bunning, P. S., and C. H. Barnett 1965 A Comparison of Adult and Foetal Talocalcaneal Articulations. Journal of Anatomy 99: 71-6.

Byers, S. N. 2008 Introduction to Forensic Anthropology. 3rd edition. Boston: Pearson/Allyn and Bacon.

Byers, S. N., K. Akoshima, and B. Curran 1989 Determination of Adult Stature from Metatarsal Length. American Journal of Physical Anthropology 79(3): 275-279.

Cameron, $\mathrm{N}$.

1984 The Measurement of Human Growth. London: Croom Helm.

Campobasso, C. P., G. Di Vella, F. Introna

1998 Using Scapular Measurements in Regression Formulae for the Estimation of Stature. Using Scapular Measurements in Regression Formulae for the Estimation of Stature. 74(7-8): 75-82. 
Carlson, K. J., F. E. Grine, and O. M. Pearson

2007 Robusticity and Sexual Dimorphism in the Postcranium of Modern HunterGatherers from Australia. American Journal of Physical Anthropology 134(1): 923.

Carson, S. A.

2010 Institutional Change, Geography, and Insolation in Nineteenth Century African-American and White Statures in Southern States. Journal of Economic Issues 44(3): 737-755.

Carty, C. L., N. A. Johnson, C. M. Hutter, A. P. Reiner, U. Peters, H. Tang, and C. Kooperberg 2012 Genome-Wide Association Study of Body Height in African Americans: The Women's Health Initiative SNP Health Association Resource (SHARe). Human Molecular Genetics 21(3): 711-720.

Chan, W.

2011 Craniofacial Differences between Modern and Archaeological Asian Skeletal Populations. Ph.D. dissertation, Ohio State University.

Charlesworth, B.

1991 The Evolution of Sex Chromosomes. Science 251(4997): 1030-1033.

Checkley, W., R. H. Gilman, R. E. Black, L. D., Epstein, L. Cabrera, C. R. Sterling 2004 Effect of Water and Sanitation on Childhood Health in a Poor Peruvian Peri-Urban Community. The Lancet 363(9403): 112-118.

Chiba, M., and K. Terazawa

1998 Estimation of Stature from Somatometry of Skull. Forensic Science International 97(2): 87-92.

Christensen, A. M.

2004 The Impact of Daubert: Implications for Testimony and Research in Forensic Anthropology (and the Use of Frontal Sinuses in Personal Identification). Journal of Forensic Sciences 49(3): 427-30.

Christensen, A. M., and C. M. Crowder 2009 Evidentiary Standards for Forensic Anthropology. Journal of Forensic Sciences 54(6): 1211-1216.

Christopher, A.J. 2001 Urban Segregation in Post-Apartheid South Africa. Urban Studies 38(3): 449-466. 
Clasen, T. F., K. Bostoen, W.P. Schmidt, S. Boisson, I. C. Fung, M. W. Jenkins, B. Scott, S. Sugden, and S. Cairncross 2010 Interventions to Improve Disposal of Human Excreta for Preventing Diarrhoea. The Cochrane Database of Systematic Reviews (6): CD007180.

Cordeiro, C., J. I. Muñoz-Barús, S. Wasterlain, E. Cunha, and D. N. Vieira 2009 Predicting Adult Stature from Metatarsal Length in a Portuguese Population. Forensic Science International 193(1): 131.e1-131.e4.

Coyle, S., and J. Kwong 2000 Women's Work and Social Reproduction in Thailand. Journal of Contemporary Asia 30(4): 492-506.

Dangour, A. D. 2001 Growth of Upper- and Lower-Body Segments in Patamona and Wapishana Amerindian Children (Cross-Sectional Data). Annals of Human Biology 28(6): 649-663.

Danubio, M. E., and E. Sanna 2008 Secular Changes in Human Biological Variables in Western Countries: An Updated Review and Synthesis. Journal of Anthropological Sciences 86: 91-112.

Darwin, C. R.

1874 The Descent of Man, and Selection in Relation to Sex. $2^{\text {nd }}$ edition. London: John Murray.

Das-Munshi, J., C. Lund, C. Mathews, C. Clark, C. Rothon, and S. Stansfeld 2016 Mental Health Inequalities in Adolescents Growing Up in Post-Apartheid South Africa: Cross-Sectional Survey, SHaW Study. PLOS ONE 11(5): e0154478.

Daubert v. Merrell Dow Pharmaceuticals, Inc., 509 U.S. 579 (1993). Accessed June 19, 2017.

De Beer, C.

1984 The South African Disease: Apartheid, Health, and Health Services. Johannesburg: Southern African Research Service.

De Groote, I., and L. T. Humphrey

2011 Body Mass and Stature Estimation Based on the First Metatarsal in Humans. American Journal of Physical Anthropology 144(4): 625-32.

de Palma, L., A. Santucci, A. Ventura, and M. Marinelli 2003 Anatomy and Embryology of the Talocalcaneal Joint. Foot and Ankle Surgery 9(1): 7-18. 
De Sanctis, V., S. Bernasconi, L. Bianchin, G. Bona, M. Bozzola, F. Buzi, C. De Sanctis, F. Rigon, L. Tatò, G. Tonini, and E. Perissinotto 2014 Onset of Menstrual Cycle and Menses Features among Secondary School Girls in Italy: A Questionnaire Study on 3,783 Students. Indian Journal of Endocrinology and Metabolism 18(Suppl 1): S84-92.

Dearden, K.A., W. Schott, B. T. Crookston, D. L. Humphries, M. E. Penny, J. R. Behrman, and The Young Lives Determinants and Consequences of Child Growth Project Team 2017 Children with Access to Improved Sanitation but Not Improved Water Are at Lower Risk of Stunting Compared to Children without Access: A Cohort Study in Ethiopia, India, Peru, and Vietnam. BMC Public Health 17(110): 1-19.

DiMichele, D. L., and M. K. Spradley 2012 Sex Estimation in a Modern American Osteological Sample Using a Discriminant Function Analysis from the Calcaneus. Forensic Science International 221(1-3): 152.e1-5.

Dirkmaat, D. C., L. L. Cabo, S. D. Ousley, and S. A. Symes 2008 New Perspectives in Forensic Anthropology. American Journal of Physical Anthropology 137(S47): 33-52.

Doak, C., L. Adair, M. Bentley, Z. Fengying, and B. Popkin 2002 The Underweight/Overweight Household: An Exploration of Household Sociodemographic and Dietary Factors in China. Public Health Nutrition 5(1A): 215-221.

Doral, M. N., M. Alam, M. Bozkurt, E. Turhan, O. A. Atay, G. Dönmez, and N. Maffulli 2010 Functional Anatomy of the Achilles Tendon. Knee Surgery, Sports Traumatology, Arthroscopy 18(5): 638-643.

Dul, J., and G. E. Johnson 1985 A Kinematic Model of the Human Ankle. Journal of Biomedical Engineering 7(2): 137-143.

Dwight, T. 1890 Sternum as an Index of Sex, Height, and Age. Journal of Anatomy and Physiology 24(Pt 4): 527-35.

Dwight, T.

1894 Methods of Estimating the Height from Parts of the Skeleton. 46: 293-296.

Eckes, T., and H. M. Trauter 2000 The Development of Social Psychology of Gender. New York: Psychology Press, Taylor and Francis Group. 
Ekizoglu, O., E. Inci, F. B. Palabiyik, I. O. Can, A. Er, M. Bozdag, I. E. Kacmaz, and E. F. Kranioti

2017 Sex Estimation in a Contemporary Turkish Population Based on CT Scans of the Calcaneus. Forensic Science International 279: 310.e1-6.

Ember, C.R., and Ember, M.

1988 Anthropology. $5^{\text {th }}$ edition. Englewood Cliffs, New Jersey: Prentice Hall.

Eshed, V., A. Gopher, E. Galili, and I. Hershkovitz

2004 Musculoskeletal Stress Markers in Natufian Hunter-Gatherers and Neolithic Farmers in the Levant: The Upper Limb. American Journal of Physical Anthropology 123(4): 303-315.

Estrada, K., M. Krawczak, S. Schreiber, K. van Duijn, L. Stolk, J. B. van Meurs, F. Liu, B. W. Penninx, J. H. Smit, N. Vogelzangs, J. J. Hottenga, G. Willemsen, E. J. de Geus, M. Lorentzon, H. von Eller-Eberstein, P. Lips, N. Schoor, V. Pop, J. de Keijzer, A. Hofman, Y. S. Aulchenko, B.A. Oostra, C. Ohlsson, D. I. Boomsma, A. G. Uitterlinden, C. M. van Dujin, F. Rivadeneira, and M. Kayser 2009 A Genome-Wide Association Study of Northwestern Europeans Involves the C-Type Natriuretic Peptide Signaling Pathway in the Etiology of Human Height Variation. Human Molecular Genetics 18(18): 3516-3524.

Faller, A., M. Schünke, and G. Schünke 2004 The Human Body: An Introduction to Structure and Function. Stuttgart; New York: Georg Thieme.

Ferguson, E. and S. Black

2011 Forensic Anthropology 2000-2010. Boca Raton: Taylor and Francis.

Figueroa-Soto, C.

2012 Sexual Dimorphism and Socioeconomic Status: Comparison between Migrant and Non-Migrant Populations. Master's Thesis, Texas State University.

Food and Agriculture Organization of the United Nations

2012 The State of Food and Agriculture. Rome, Italy: Food and Agriculture Organization of the United Nations.

Fox, K., and T. B. Heaton

2012 Child Nutritional Status by Rural/Urban Residence: A Cross-National Analysis. The Journal of Rural Health: Official Journal of the American Rural Health Association and the National Rural Health Care Association 28(4): 380391.

Franklin, D., A. Cardini, A. Flavel, and A. Kuliukas 2013 Estimation of Sex from Cranial Measurements in a Western Australian Population. Forensic Science International 229(1-3): 158.e1-8. 
Frayer, D. W.

1980 Sexual Dimorphism and Cultural Evolution in the Late Pleistocene and Holocene of Europe. Journal of Human Evolution 9(5): 399-415.

Frayer, D.W.

1981 Body Size, Weapon Use, and Natural Selection in the European Upper Paleolithic and Mesolithic. American Anthropologist 83(1): 57-73.

Frayer, D. W, and M. H Wolpoff

1985 Sexual Dimorphism. Annual Review of Anthropology 14(1): 429-473.

Fritsch, H., and R. Eggers

1999 Ossification of the Calcaneus in the Normal Fetal Foot and in Clubfoot. Journal of Pediatric Orthopaedics 19(1): 22-26.

Fritsch, H., E. Brenner, and P. Debbage 2001 Ossification in the Human Calcaneus: A Model for Spatial Bone Development and Ossification. Journal of Anatomy 199(5): 609-616.

Frye v. United States, 54 App.D.C. 46, 293 F. 1013 1923. Accessed June 19, 2017.

Fully, G.

1956 Une Nouvelle Methode de Determination de La Taille. Annals De Medecine Legale 35: 266-273.

Gajdos, Z. K., J. N. Hirschhorn, and M. R. Palmert 2009 What Controls the Timing of Puberty? An Update on Progress from Genetic Investigation. Current Opinion in Endocrinology, Diabetes, and Obesity 16(1): $16-24$.

Gajdos, Z. K., K. D. Henderson, J. N. Hirschhorn, and M. R. Palmert 2010 Genetic Determinants of Pubertal Timing in the General Population. Molecular and Cellular Endocrinology 324(1-2): 21-29.

Gardner, E., R. O’Rahilly, and D. J. Gray 1959 The Prenatal Development of the Skeleton and Joints of the Human Foot. Journal of Bone and Joint Surgery 41(5): 847-76.

Giles, E., and Elliot, O. 1962 Race Identification from Cranial Measurements. Journal of Forensic Sciences 7 (2): 147-157.

Giles, E., and Klepinger, L.L. 1988 Confidence Intervals for Estimates Based on Linear Regression in Forensic Anthropology 33(5): 1218-1222. 
Gill, C. M., A. K. Taneja, M. A. Bredella, M. Torriani, and J. M. Desilva 2014 Osteogenic Relationship between the Lateral Plantar Process and the Peroneal Tubercle in the Human Calcaneus. Journal of Anatomy 224(2): 173179.

Gill, G. W.

1995 Challenge on the Frontier: Discerning American Indians from Whites Osteologically. Journal of Forensic Sciences 40(5): 783-8.

Gill, G.W., and Rhine, S. 1990 Skeletal Attribution of Race: Methods for Forensic Anthropology. Anthropological Papers, 4. Albuquerque, NM: Maxwell Museum of Anthropology.

Gilmour, J., and D. Skuse 1999 A Case-Comparison Study of the Characteristics of Children with a Short Stature Syndrome Induced by Stress (Hyperphagic Short Stature) and a Consecutive Series of Unaffected "Stressed" Children. Journal of Child Psychology and Psychiatry, and Allied Disciplines 40(6): 969-978.

Giroux, C. L., and D. J. Wescott 2008 Stature Estimation Based on Dimensions of the Bony Pelvis and Proximal Femur. Journal of Forensic Sciences 53(1): 65-68.

Giurazza, F., R. Del Vescovo, E. Schena, R. L. Cazzato, F. D’Agostino, R. F. Grasso, S. Silvestri, and B. B. Zobel 2013 Stature Estimation from Scapular Measurements by CT Scan Evaluation in an Italian Population. Legal Medicine 15(4): 202-208.

Giurazza, F., R. Del Vescovo, E. Schena, S. Battisti, R. L. Cazzato, F. R. Grasso, S. Silvestri, V. Denaro, and B. B. Zobel 2012 Determination of Stature from Skeletal and Skull Measurements by CT Scan Evaluation. Forensic Science International 222(1-3): 398.e1-398.e9.

Gocha, T. P., G. Vercellotti, L. E. Mccormick, and T. L. Van Deest 2013 Formulae for Estimating Skeletal Height in Modern South-East Asians. Journal of Forensic Sciences 58(5): 1279-1283.

González-Colmenares, G., C. S Medina, and L. C. Báez 2016 Estimation of Stature by Cephalometric Facial Dimensions in Skeletonized Bodies: Study from a Sample Modern Colombians Skeletal Remains. Forensic Science International 258: 101.e1-101.e6. 
Gorai, I., K. Tanaka, M. Inada, H. Morinaga, Y. Uchiyama, R. Kikuchi, O. Chaki, and F. Hirahara

2003 Estrogen-Metabolizing Gene Polymorphisms, But Not Estrogen Receptor- $\alpha$ Gene Polymorphisms, Are Associated with the Onset of Menarche in Healthy Postmenopausal Japanese Women. The Journal of Clinical Endocrinology \& Metabolism 88(2): 799-803.

Government of Canada

2014 Canada’s Missing. http://www.canadasmissing.ca, accessed April 3, 2017.

Graves, J. A. M.

1995 The Evolution of Mammalian Sex Chromosomes and the Origin of Sex Determining Genes [and Discussion]. Philosophical Transactions: Biological Sciences 350(1333): 305-312.

Graw, M., A. Czarnetzki, and H. T. Haffner 1999 The Form of the Supraorbital Margin as a Criterion in Identification of Sex from the Skull: Investigations Based on Modern Human Skulls. American Journal of Physical Anthropology 108(1): 91-6.

Greulich, W. W.

1951 The Growth and Developmental Status of Guamanian School Children in 1947. American Journal of Physical Anthropology 9(1): 55-70.

Gualdi Russo, E. 2007 Sex Determination from the Talus and Calcaneus Measurements. Forensic Science International 171(2-3): 151-156.

Guenoun, B., F. Zadegan, F. Aim, D. Hannouche, and R. Nizard 2012 Reliability of a New Method for Lower-Extremity Measurements Based on Stereoradiographic Three-Dimensional Reconstruction. Orthopaedics \& Traumatology, Surgery \& Research: OTSR 98(5): 506-513.

Habib, S. R., and N. N. Kamal 2010 Stature Estimation from Hand and Phalanges Lengths of Egyptians. Journal of Forensic and Legal Medicine 17(3): 156-160.

Hall R. L., and M. J. Shereff 1993 Anatomy of the Calcaneus. Clinical Orthopaedics and Related Research (290): 27-35.

Hamilton, M. E.

1975 Variation Among Five Groups of Amerindians in the Magnitude of Sexual Dimorphism of Skeletal Size. Ann Arbor: University Microfilms. 
Hannaford, I.

1996 Race: The History of an Idea in the West. Washington, D.C. : Woodrow Wilson Center Press; Baltimore, Maryland: Order from the Johns Hopkins University Press.

Hao, Y., X. Liu, X. Lu, X. Yang, L. Wang, S. Chen, H. Li, J. Li, J. Cao, J. Chen, Y. Li, L. Zhao, Y. Shi, C. Shen, W. Yan, J. He, J. Huang, and D. Gu 2013 Genome-Wide Association Study in Han Chinese Identifies Three Novel Loci for Human Height. Human Genetics 132(6): 681-689.

Haque, R.

2007 Human Intestinal Parasites. Journal of Health, Population, and Nutrition 25(4): 387-391.

Harrison, G. P. 2010 Race and Reality: What Everyone Should Know about Our Biological Diversity. Amherst, New York: Prometheus Books.

Hasselwander, A. 1903 Untersuchungen über die Ossification des Menschlichen Fussskelets. Zeitschrift für Morphologie und Anathropologie 5: 438-508.

Hathi, P., S. Haque, L. Pant, D. Coffey, and D. Spears 2017 Place and Child Health: The Interaction of Population Density and Sanitation in Developing Countries. Demography 54(1): 337-360.

Heaton, T. B., and R. Forste 2003 Rural/Urban Differences in Child Growth and Survival in Bolivia. Rural Sociology 68(3): 410-433.

Henke, W., and C. Reyher, 1874 Studien Uber Die Entwicklung der Extremitaten des Menschen, Insbesondere der Gelenkflachen. 70: 217-273.

Hicks, J. H.

1953 The Mechanics of the Foot. I. The Joints. Journal of Anatomy 87(4): 34557.

Hicks, J. H.

1954 The Mechanics of the Foot. II. The Plantar Aponeurosis and the Arch. Journal of Anatomy 88(1): 25-30.

Hintzsche, E. 1930 Beitrag Zur Entwicklung des Menschlichen Fersenbeins. 21: 531-551. 
Hofmeister, E. P., P. Juliano, and F. Lippert

1996 The Anatomical Configuration and Clinical Implications of the Peroneal Tubercle. The Foot 6(3): 138-142.

Holden, C., and R. Mace 1999 Sexual Dimorphism in Stature and Women's Work: A Phylogenetic CrossCultural Analysis. American Journal of Physical Anthropology 110(1): 27-45.

Holland, T. D. 1995 Estimation of Adult Stature from the Calcaneus and Talus. American Journal of Physical Anthropology 96(3): 315-320.

Holliday, T. W., and C. B. Ruff

1997 Ecogeographical Patterning and Stature Prediction in Fossil Hominids: Comment on M. R. Feldesman and R. L. Fountain, American Journal of Physical Anthropology (1996) 100:207-224. American Journal of Physical Anthropology 103(1): 137-140.

Holobinko, A.

2012 Forensic Human Identification in the United States and Canada: A Review of the Law, Admissible Techniques, and the Legal Implications of Their Application in Forensic Cases. Forensic Science International 222(1-3): 394.e1394.e13.

Hong, R., J. E. Banta, and J. A. Betancourt 2006 Relationship between Household Wealth Inequality and Chronic Childhood Under-Nutrition in Bangladesh. International Journal for Equity in Health 5: 15.

Humphrey, L. T. 1998 Growth Patterns in the Modern Human Skeleton. American Journal of Physical Anthropology 105(1): 57-72.

Hunt, L. M., and M. S. Megyesi 2008 The Ambiguous Meanings of the Racial/Ethnic Categories Routinely Used in Human Genetics Research. Social Science \& Medicine 66(2): 349-361.

Hyer, C. F., J. M. Dawson, T. M. Philbin, G. C. Berlet, and T. H. Lee 2005 The Peroneal Tubercle: Description, Classification, and Relevance to Peroneus Longus Tendon Pathology. Foot \& Ankle International 26(11): 947950.

Inkster, R. G.

1927 The Form of the Talus with Special Reference to That of the Australian Aborigine. Doctor of Medicine Thesis, University of Edinburgh. 
INTERPOL

2014 INTERPOL Disaster Victim Identification Guide. Accessed February 28, 2017. https://www.interpol.int/INTERPOL-expertise/Forensics/DVI

Introna, F., G. Di Vella, C. P. Campobasso, and M. Dragone 1997 Sex Determination by Discriminant Analysis of Calcanei Measurements. Journal of Forensic Sciences 42(4): 725-728.

Introna, F., G. Di Vella, and S. Petrachi 1993 Determination of Height in Life using Multiple Regression of Skull Parameters. Bollettino Della Societa Italiana Di Biologia Sperimentale 69(3): $153-160$.

Inwood, K., and E. Roberts 2010 Longitudinal Studies of Human Growth and Health: A Review of Recent Historical Research. Journal of Economic Surveys 24(5): 801-840

Intergovernmental Panel on Climate Change (IPCC) 2001 Climate Change 2001: The Scientific Basis. Contribution of Working Group I to the Third Assessment Report of the Intergovernmental Panel on Climate Change. J. T. Houghton, Y. Ding, D. J. Griggs, N. Noguer, P. J. van Der Linden, D. Xiaosu, K. Maskell and C. A. Johnson., eds. Cambridge., United Kingdom; New York, United States of America: Cambridge University Press

Intergovernmental Panel on Climate Change (IPCC) 2007 Climate Change 2007: Synthesis Report. Contribution of Working Groups I, II, and III to the Fourth Assessment Report of the Intergovernmental Panel on Climate Change. Core Writing Team, R. K. Pachauri, and A. Reisinger, eds. Geneva, Switzerland: IPCC.

Isman, R. E., and V. T. Inman 1969 Anthropometric Studies of the Human Foot and Ankle 11: 97-129.

Isvilanonda, S, and I Bunyasiri 2009 Food Security in Thailand: Status, Rural Poor Vunerability, and Some Policy Options. ARE Working Paper, 2552/1. Bangkok: Department of Agricultural and Resource Economics, Faculty of Economics, Kasetsart University.

Jacobs, P. A., and J. A. Strong 1959 A Case of Human Intersexuality Having a Possible XXY Sex-Determining Mechanism. Nature 183(4657): 302-303.

Jagadish Rao, P. P., J. Sowmya, K. Yoganarasimha, R. G. Menezes, T. Kanchan, and R. Aswinidutt 2009 Estimation of Stature from Cranial Sutures in a South Indian Male Population. International Journal of Legal Medicine 123(3): 271-276. 
James, $\mathrm{H}$.

2005 Thai Tsunami Victim Identification Overview to Date. The Journal of Forensic Odonto-Stomatology 23(1): 1-18.

Jarungrattanapong, R., and A. Manasboonphempool

2011 Adaptive Capacity of Households and Institutions in Dealing with Floods in Chiang Mai, Thailand. Economy and Environment Program for Southeast Asia (EEPSEA)

Report No. 2011-RR2. Singapore: Economy and Environment Program for Southeast Asia.

Jason, D. R., and K. Taylor 1995 Estimation of Stature from the Length of the Cervical, Thoracic, and Lumbar Segments of the Spine in American Whites and Blacks. Journal of Forensic Sciences 40(1): 59-62.

Jasuja, O. P., and Singh, G. 2004 Estimation of Stature from Hand and Phalange Length. JIAFM 26(3): 0971-0973.

Jee, S. C., and M. H. Yun 2015 Estimation of Stature from Diversified Hand Anthropometric Dimensions from Korean Population. Journal of Forensic and Legal Medicine 35: 9-14.

Jellema, L. M.

2017 Personal Communication, January 10, 2017.

Jinsart, W., and S. Thepanondh

2014 Effects of Climate Change on Heat Accumulation and Precipitation in Thailand. International Journal of Environmental Science and Development 5(4): 340-343.

Jit, I., and S. Singh

1956 Estimation of Stature from Clavicles. The Indian Journal of Medical Research 44(1): 137-155.

Johansson, A., F. Marroni, C. Hayward, C. S. Franklin, A. V. Kirichenko, I. Johansson, A. A. Hicks, V. Vitart, A. Isaacs, T. Axenovich, S. Campbell, M. G. Dunlop, J. Floyd, N. Hastie, A. Hofman, S. Knott, I. Kolcic, I. Pichler, O. Polasek, F. Rivadeneira, A. Tenesa, A. G. Uitterlinden, S. H. Wild, I. V. Zorkoltseva, T. Meitinger, J. F. Wilson, I. Rudan, H. Campbell, C. Pattaro, P. Pramstaller, B. A. Oostra, A. F. Wright, C. M. van Duijn, Y. S. Aulchenko, and U. Gyllensten 2009 Common Variants in the JAZF1 Gene Associated with Height Identified by Linkage and Genome-Wide Association Analysis. Human Molecular Genetics 18(2): 373-380. 
Jorde, L. B., and Wooding, S. P. 2004 Genetic Variation, Classification and "Race". Nature Genetics 36(11): S2833.

Jothityangkoon, C., C. Hirunteeyakul, K. Boonrawd, and M. Sivapalan 2013 Assessing the Impact of Climate and Land Use Changes on Extreme Floods in a Large Tropical Catchment. Journal of Hydrology 490: 88-105.

Kahana, T., and J. Hiss 2009 The Role of Forensic Anthropology in Mass Fatality Incidents Management. Forensic Science Policy and Management: An International Journal 1(3): 144-149.

Kalichman, S. C., L. C. Simbayi, M. Kaufman, D. Cain, and S. Jooste 2007 Alcohol Use and Sexual Risks for HIV/AIDS in Sub-Saharan Africa: Systematic Review of Empirical Findings. Prevention Science 8(2): 141-151.

Kalichman, S. C., L. C. Simbayi, R. Vermaak, S. Jooste, and D. Cain 2008 HIV/AIDS Risks among Men and Women Who Drink at Informal Alcohol Serving Establishments (Shebeens) in Cape Town, South Africa. Prevention Science 9(1): 55-62.

Karaan, A. S. M., and A. S. Myburgh 1992 Food Distribution Systems in the Urban Informal Markets: The Case of Red Meat Marketing in the Western Cape Townships and Informal Settlements. Agricultural Economics Research, Policy and Practice in Southern Africa 31(4): 289-293.

Karakas, H., O. Celbis, A. Harma, and B. Alicioglu 2011 Total Body Height Estimation Using Sacrum Height in Anatolian Caucasians: Multidetector Computed Tomography-Based Virtual Anthropometry. Skeletal Radiology 40(5): 623-630.

Kark, S. L.

1974 Epidemiology and Community Medicine. New York: Apple Century Crofts.

Keener, B. J., and J. A. Sizensky 2005 The Anatomy of the Calcaneus and Surrounding Structures. Foot and Ankle Clinics of North America 10(3): 413-424.

Kennedy, G., N. Fanou, C. Seghieri, and I. D. Brouwer 2009 Dietary Diversity as a Measure of the Micronutrient Adequacy of Women's Diets: Results from Bamako, Mali Site. FHI 360. Washington: DC: Food and Nutrition Technical Assistance II Project. 
Kennedy, K. A. R.

1989 Skeletal Markers of Occupational Stress. In Iscan MY, Kenneth AR, Editors. Reconstruction of Life from the Skeleton. Pp. 129-160. New York: Alan R. Liss.

Kennedy, K. A. R.

1995 But Professor, Why Teach Race Identification If Races Don't Exist? Journal of Forensic Science 40(5): 797-800.

Khanpetch, P., S. Prasitwattanseree, D. T. Case, and P. Mahakkanukrauh 2012 Determination of Sex from the Metacarpals in a Thai Population. Forensic Science International 217(1-3): 229.e1-229.e8.

Kim, D. I., Y. S. Kim, U. Y. Lee, and S. H. Han 2013 Sex Determination from Calcaneus in Korean Using Discriminant Analysis. Forensic Science International 228(1): 177.e1-177.e7.

Kimura, K.

1992 Estimation of Stature from Second Metacarpal Length in Japanese Children. Annals of Human Biology 19(3): 267-75.

Kinaston, R. L., R. K. Walter, C. Jacomb, E. Brooks, N. Tayles, S. E. Halcrow, C. Stirling, M. Reid, A. R. Gray, J. Spinks, B. Shaw, R. Fyfe, and H. R. Buckley 2013 The First New Zealanders: Patterns of Diet and Mobility Revealed through Isotope Analysis. PLOS ONE 8(5): e64580.

King, C. A., M. Y. Işcan, and S. R. Loth 1998 Metric and Comparative Analysis of Sexual Dimorphism in the Thai Femur. Journal of Forensic Sciences 43(5): 954-8.

King, K. A. 2004 A Test of the Fully Anatomical Method of Stature Estimation. American Journal of Physical Anthropology: 125-125.

Knight, B., B. M. Shields, M. Turner, R. J. Powell, C. S. Yajnik, and A. T. Hattersley 2005 Evidence of Genetic Regulation of Fetal Longitudinal Growth. Early Human Development 81(10): 823-831.

Komar, D. A., and C. Grivas 2008 Manufactured Populations: What Do Contemporary Reference Skeletal Collections Represent? A Comparative Study Using the Maxwell Museum Documented Collection. American Journal of Physical Anthropology 137(2): 224-233. 
Kon, Z. R., and N. Lackan

2008 Ethnic Disparities in Access to Care in Post-Apartheid South Africa.

American Journal of Public Health 98(12): 2272-2277.

Konigsberg, L. W., B. F. B. Algee-Hewitt, and D. W. Steadman

2009 Estimation and Evidence in Forensic Anthropology: Sex and Race.

American Journal of Physical Anthropology 139(1): 77-90.

Konigsberg, L. W., S. H. Hens

1998 Use of Ordinal Categorical Variables in Skeletal Assessment of Sex from the Cranium. American Journal of Physical Anthropology 107: 97-112.

Kottak, C. P.

2011 Anthropology: Appreciating Human Diversity. New York: McGraw-Hill.

Kranioti, E., and R. Paine

2011 Forensic Anthropology in Europe: An Assessment of Current Status and Application. Journal of Anthropological Sciences 89: 71-92.

Krishan, K.

2008 Estimation of Stature from Cephalo-Facial Anthropometry in North Indian Population. Forensic Science International 181(1): 52.e1-52.e6.

Krishan, K., and R. Kumar 2007 Determination of Stature from Cephalo-Facial Dimensions in a North Indian Population. Legal Medicine 9(3): 128-133.

Lagravère, M. O., C. Low, C. Flores-Mir, R. Chung, J. P. Carey, G. Heo, and P. W. Major 2010 Intraexaminer and Interexaminer Reliabilities of Landmark Identification on Digitized Lateral Cephalograms and Formatted 3-Dimensional Cone-Beam Computerized Tomography Images. American Journal of Orthodontics and Dentofacial Orthopedics 137(5): 598-604.

Langley N. R., and M. T. Tersigni-Tarrant 2017 Forensic Anthropology: An Introduction. Boca Raton, Florida: CRC Press.

Latimer, B., and C. O. Lovejoy 1989 The Calcaneus of Australopithecus Afarensis and Its Implications for the Evolution of Bipedality. American Journal of Physical Anthropology 78(3): 369386.

Lazarus, R. S., and S. Folkman 1984 Stress, Appraisal, and Coping. New York: Springer Publishing Company. 
Leardini, A., R. Stagni, and J. J. O’Connor 2001 Mobility of the Subtalar Joint in the Intact Ankle Complex. Journal of Biomechanics 34(6): 805-809.

Leboucq, $\mathrm{H}$. 1886 Sur la Morphologie du Carpe et du Tarse. Anatomischer Anzeiger 1: 17-21.

Lee, M. H, S. H. Kim, M. Oh, K. W. Lee, and M. J. Park 2016 Age at Menarche in Korean Adolescents: Trends and Influencing Factors. Reproductive Health 13(121): 1-7.

Lee, U. Y., S. H. Han, D. K. Park, Y. S. Kim, D. I. Kim, I. H. Chung, and M. H. Chun 2012 Sex Determination from the Talus of Koreans by Discriminant Function Analysis. Journal of Forensic Sciences 57(1): 166-171.

Lei, S. F., T. L. Yang, L. J. Tan, X. D. Chen, Y. Guo, L. Zhang, X. G. Liu, H. Yan, F. Pan, Z. X. Zhang, Y. M. Peng, Q. Zhou, L. N. He, X. Z. Zhu, J. Cheng, Y. Z. Liu, C. J. Papasian, and H. Q. Deng 2009 Genome-Wide Association Scan for Stature in Chinese: Evidence for Ethnic Specific Loci. Human Genetics 125(1): 1-9.

Leon, D. A.

2008 Cities, Urbanization and Health. International Journal of Epidemiology 37(1): 4-8.

Lesciotto, K. M. 2015 The Impact of Daubert on the Admissibility of Forensic Anthropology Expert Testimony. Journal of Forensic Sciences 60(3): 549-555.

Lettre, G., A. U. Jackson, C. Gieger, F. R. Schumacher, S. I. Berndt, S. Sanna, S. Eyheramendy, B. F. Voight, J. L. Butler, C. Guiducci, T. Illig, R. Hackett, I. M. Heid, K. B. Jacobs, V. Lyssenko, M. Uda, Diabetes Genetics Initiative, FUSION, KORA, Prostate, Lung, Colorectal and Ovarian Cancer Screening Trial, Nurses' Health Study, SardiNIA, M. Boehnke, S. J. Chanock, L. C. Groop, F. B. Hu, B. Isomaa, P. Kraft, L. Peltonen, V. Salomaa, D. Schlessinger, D. J. Hunter, R. B. Hayes, G. R. Abecasis, H. E. Wichmann, K. L. Mohlke, and J. N. Hirschhorn 2008 Identification of Ten Loci Associated with Height Highlights New Biological Pathways in Human Growth. Nature Genetics 40(5): 584-591.

Lewis, B. A. 1998 Prehistoric Juvenile Rheumatoid Arthritis in a Precontact Louisiana Native Population Reconsidered. American Journal of Physical Anthropology 106: 229248.

Lewontin, R. C. 1972 The Apportionment of Human Diversity. Evolutionary Biology 6: 381-398. 
Limaye, R. J., B. Srirojn, P. Lilleston, A. Aramrattana, N. Thomson, D. D. Celentano, and S. G. Sherman

2013 A Qualitative Exploration of the Effects of Increasing the Minimum

Purchase Age of Alcohol in Thailand. Drug and Alcohol Review 32(1): 100-105.

Lin, L. I.

1989 A Concordance Correlation Coefficient to Evaluate Reproducibility.

Biometrics 45(1): 255-268.

Little, W., and R. McGivern

2014 Introduction to Sociology. 1st Canadian Edition. OpenStax College: BC Open Textbook Project.

Liu, J. Z., S. E. Medland, M. J. Wright, A. K. Henders, A. C. Heath, P. A. Madden, A. Duncan, G. W. Montgomery, N. G. Martin, and A. F. McRae 2010 Genome-Wide Association Study of Height and Body Mass Index in Australian Twin Families. Twin Research and Human Genetics 13(2): 179-193.

Loesch, D. Z., J. L. Hopper, E. Rogucka, and R. M. Huggins 1995 Timing and Genetic Rapport between Growth in Skeletal Maturity and Height around Puberty: Similarities and Differences between Girls and Boys. American Journal of Human Genetics 56(3): 753-759.

Lopez, T. T., E. Michel-Crosato, E. de Novaes Benedicto, L. A. S. de Paiva, and M. G. H. Biazevic 2017 Accuracy of Mandibular Measurements of Sexual Dimorphism Using Stabilizer Equipment. Brazilian Oral Research 31: e1-11.

Lundy, J.K.

1983 Selected Aspects of Metrical and Morphological Infracranial Skeletal Variation in the South African Negro. Ph. D dissertation, University of the Witwatersrand.

Luong, T.V., O. Chanacharnmonkol, and T. Thatsanatheb 2010 (2000) Universal Sanitation - Thailand Experiences. In 26th WEDC Conference Proceedings: Water, Sanitation, and Hygiene: Challenges of the Millennium. Dhaka, Bangladesh.

Macgregor, S., B. K. Cornes, N. G. Martin, and P. M. Visscher 2006 Bias, Precision and Heritability of Self-Reported and Clinically Measured Height in Australian Twins. Human Genetics 120(4): 571-580.

Magnani, R. J., N. B. Mock, W. E. Bertrand, and D. C. Clay 1993 Breast-Feeding, Water and Sanitation, and Childhood Malnutrition in the Philippines. Journal of Biosocial Science 25(2): 195-211. 
Mahakkanukrauh, P., P. Khanpetch, S. Prasitwattanseree, K. Vichairat, and D. T. Case 2011 Stature Estimation from Long Bone Lengths in a Thai Population. Forensic Science International 210(1-3): 279.e1-279.e7.

Mahakkanukrauh, P., S. Praneatpolgrang, S. Ruengdit, P. Singsuwan, P. Duangto, and D. T. Case 2014 Sex Estimation from the Talus in a Thai Population. Forensic Science International. 240: 152.e1-152.e8.

Mahakkanukrauh, P., S. Ruengdit, S. M. Tun, D. T. Case, and A. Sinthubua 2017 Osteometric Sex Estimation from the Os Coxa in a Thai Population. Forensic Science International 271: 127.e1-127.e7.

Mahalakshmi, I. P., and P. M. David 2013 Determination of Sexual Dimorphism and Stature from Lateral Cephalogram. Journal of Indian Academy of Oral Medicine and Radiology 25(2): 116-120.

Mahato, N. K. 2011 Morphology of Sustentaculum Tali: Biomechanical Importance and Correlation with Angular Dimensions of the Talus. The Foot 21(4): 179-183.

Mahato, N. K., and S. N Murthy 2012 Articular and Angular Dimensions of the Talus: Inter-Relationship and Biomechanical Significance. The Foot 22(2): 85-89.

Major, P. W., D. E. Johnson, K. L. Hesse, and K. E. Glover 1994 Landmark Identification Error in Posterior Anterior Cephalometrics. The Angle Orthodontist 64(6): 447-454.

Malina, R. M., J. H. Himes, C. D. Stepick, F. G. Lopez, and P. H. Buschang 1981 Growth of Rural and Urban Children in the Valley of Oaxaca, Mexico. American Journal of Physical Anthropology 55(2): 269-280.

Manter, J. T.

1941 Movements of the Subtalar and Transverse Tarsal Joints. Anatomical Record 80(4): 397-410.

Marinho, L., D. Almeida, A. Santos, and H. F. V. Cardoso 2012 Is the Length of the Sternum Reliable for Estimating Adult Stature? A Pilot Study Using Fresh Sterna and a Test of Two Methods Using Dry Sterna. Forensic Science International 220(1-3): 292.e1-292.e4. 
Mariotti, M.

2012 Labour Markets during Apartheid in South Africa. The Economic History Review 65(3): 1100-1122.

Marks, D.

2011 Climate Change and Thailand: Impact and Response. Contemporary

Southeast Asia: A Journal of International and Strategic Affairs 33(2): 229-258.

Marlowe, F. W.

2007 Hunting and Gathering. Cross-Cultural Research 41(2): 170-195.

Martin, R, and R Knußmann

1988 Anthropologie: Handbuch Der Vergleichenden Biologie des Menschen.

Stuttgart: Gustav Fischer.

Martini, F., and J. L. Nath

2009 Fundamentals of Anatomy $\&$ Physiology. $8^{\text {th }}$ edition. San Francisco:

Pearson/Benjamin Cummings.

Masten, J., and J. J. Strzelczyk

2001 Admissibility of Scientific Evidence Post-Daubert. Health Physics 81(6):

678-82.

Meadows, L., and R. L. Jantz

1992 Estimation of Stature from Metacarpal Lengths. Journal of Forensic

Sciences 37(1): 147-54.

Meadows, L., and R. L. Jantz

1999 Secular Change in Long Bone Length and Proportion in the United States, 1800-1970. American Journal of Physical Anthropology 110: 57-67.

Meek, S.

2017 Personal Communication, August 1, 2017.

Meindl, R. S., C. O. Lovejoy, R. P. Mensforth, and L. D. Carlos 1985 Accuracy and Direction of Error in the Sexing of the Skeleton: Implications for Paleodemography. American Journal of Physical Anthropology 68(1): 79-85.

Menezes, R. G., T. Kanchan, G. P. Kumar, et al. 2009 Stature Estimation from the Length of the Sternum in South Indian Males: A Preliminary Study. Journal of Forensic and Legal Medicine 16(8): 441-443.

Menezes, R. G., K. R. Nagesh, F. N. P. Monteiro, G. P. Kumar, T. Kanchan, S. Uysal, P. P. Jagadish Rao, P. Rastogi, S. W. Lobo, and S. G. Kalthur 2011 Estimation of Stature from the Length of the Sternum in South Indian Females. Journal of Forensic and Legal Medicine 18(6): 242-245. 
Merchant, A. T., C. Jones, A. Kiure, R. Kupka, G. Fitzmaurice, M. G. Herrera, and W. W. Fawzi

2003 Water and Sanitation Associated with Improved Child Growth. European Journal of Clinical Nutrition 57(12): 1562-1568.

Meredith, $\mathrm{H}$.

1944 Human Foot Length from Embryo to Adult. Human Biology 16(4): 207282.

Molnar, S.

1983 Human Variation: Races, Types and Ethnic Groups. Englewood Cliffs, New Jersey: Prentice-Hall.

Montagu, A.

1997 Man's Most Dangerous Myth: The Fallacy of Race. $6^{\text {th }}$ edition. Walnut Creek, California: AltaMira Press.

Moore, M. K., E. A. DiGangi, F. P. N. Ruíz, O. J. H. Davila, and C. S. Medina 2016 Metric Sex Estimation from the Postcranial Skeleton for the Colombian Population. Forensic Science International 262: 286.e1-8.

Mukhopadhyay, C. C., and Y. T. Moses

1997 Re-establishing "Race" in Anthropological Discourse. American Anthropologist 99(3): 517-533.

Müller, G.

1935 Zur Bestimmung der Länge beschädigter Extremitätenknochen.

Anthropologischer Anzeiger 12: 70-72.

Mundorff, A.

2012 Integrating Forensic Anthropology into Disaster Victim Identification.

Forensic Science, Medicine, and Pathology 8(2): 131-139.

Murdock, G. P., and C. Provost

1973 Factors in the Division of Labor by Sex: A Cross-Cultural Analysis.

Ethnology 12(2): 203-225.

Murphy, A.M.C.

2002a The Calcaneus: Sex Assessment of Prehistoric New Zealand Polynesian

Skeletal Remains 1(29): 205-208.

Murphy, A.M.C.

2002b The Talus: Sex Assessment of Prehistoric New Zealand Polynesian Skeletal Remains. Forensic Science International 128(3): 155-158. 
Murphy, A.M.C.

2005 The Articular Surfaces of the Hindfoot: Sex Assessment of Prehistoric New Zealand Polynesian Skeletal Remains. Forensic Science International 151(1): 1922.

Musgrave, J. H., and N. K. Harneja

1978 The Estimation of Adult Stature from Metacarpal Bone Length. American Journal of Physical Anthropology 48(1): 113-119.

Myint tun, S., S. Das, S. Ruengdit, P. Singsuwan, and P. Mahakkanukrauh 2015 Sex Determination from Different Sternal Measurements: A Study in a Thai Population. Journal of the Anatomical Society of India 64(2): 155-161.

Nagesh, K. R., and P.G. Kumar 2006 Estimation of Stature from Vertebral Column Length in South Indians. Legal Medicine 8(5): 269-272.

Nathena, D., E. Michopoulou, and E. F. Kranioti 2017 Sexual Dimorphism of the Calcaneus in Contemporary Cretans. Forensic Science International 277: 260.e1-260.e8.

Naude, C. E., P. D. Carey, R. Laubscher, G. Fein, and M. Senekal 2012 Vitamin D and Calcium Status in South African Adolescents with Alcohol Use Disorders. Nutrients 4(8): 1076-1094.

Nicholas, S., and D. Oxley 1993 The Living Standards of Women during the Industrial Revolution, 17951820. The Economic History Review 46(4): 723-749.

Noipayak, P., P. Rawdaree, B. Supawattanabodee, and S. Manusirivitthaya 2017 Factors Associated with Early Age at Menarche among Thai Adolescents in Bangkok: A Cross-Sectional Study. BMC Women's Health 17(16): 1-6.

Norman, G., S. Pedley, and B. Takkouche 2010 Effects of Sewerage on Diarrhoea and Enteric Infections: A Systematic Review and Meta-Analysis. The Lancet. Infectious Diseases 10(8): 536-544.

Nyati, L. H., S. A. Norris, N. Cameron, and J. M. Pettifor 2006 Effect of Ethnicity and Sex on the Growth of the Axial and Appendicular Skeleton of Children Living in a Developing Country. American Journal of Physical Anthropology 130(1): 135-141.

Ocha, W.

2012 Transsexual Emergence: Gender Variant Identities in Thailand. Culture, Health \& Sexuality 14(5): 563-575. 
Otong, E. S, A. A. Rufai, D. S. Amaza, Z. Mustapha, T. W. Jacks, and M. Attah 2016 Estimation of Sex Using Antero-Posterior Dimension of Talus in North Eastern Nigerians. British Journal of Medicine and Medical Research 14(3): 1-8.

Ousley, S., Jantz, R., and Freid, D.

2009 Understanding Race and Human Variation: Why Forensic Anthropologists Are Good at Identifying Race. American Journal of Physical Anthropology 139(1): 68-76.

Oygucu, I. H., M. A. Kurt, I. Ikiz, T. Erem, and D. C. Davies 1998 Squatting Facets on the Neck of the Talus and Extensions of the Trochlear Surface of the Talus in Late Byzantine Males. Journal of Anatomy 192: 287-291.

Pablos, A., A. Gómez-Olivencia, A. García-Pérez, I. Martínez, C. Lorenzo, and J. L. Arsuaga 2013 From Toe to Head: Use of Robust Regression Methods in Stature Estimation Based on Foot Remains. Forensic Science International 226: 299.e1299.e7.

Paciorek, C. J., G. A. Stevens, M. M. Finucane, M. Ezzati, and Nutrition Impact Model Study Group 2013 Children's Height and Weight in Rural and Urban Populations in LowIncome and Middle-Income Countries: A Systematic Analysis of PopulationRepresentative Data. The Lancet. Global Health 1(5): e300-309.

Pandey, S. K., and S. Singh 1990 Study of Squatting Facet/Extension of Talus in Both Sexes. Medicine, Science and the Law 30(2): 159-164.

Panter-Brick, C., A. Todd, and R. Baker 1996 Growth Status of Homeless Nepali Boys: Do They Differ from Rural and Urban Controls? Social Science \& Medicine (1982) 43(4): 441-451.

Parent, A. S., G. Teilmann, A. Juul, N. E. Skakkebaek, J. Toppari, and J. P. Bourguignon 2003 The Timing of Normal Puberty and the Age Limits of Sexual Precocity: Variations around the World, Secular Trends, and Changes after Migration. Endocrine Reviews 24(5): 668-693.

Patil, K. R., and R. N. Mody 2005 Determination of Sex by Discriminant Function Analysis and Stature by Regression Analysis: A Lateral Cephalometric Study. Forensic Science International 147(2-3): 175-80.

Patriquin, M. L, M Steyn, and S. R Loth 2002 Metric Assessment of Race from the Pelvis in South Africans. Forensic Science International 127(1-2): 104-113. 
Peckmann, T. R., K. Orr, S. Meek, and S. K. Manolis 2015a Sex Determination from the Calcaneus in a 20th Century Greek Population Using Discriminant Function Analysis. Science \& Justice: Journal of the Forensic Science Society 55(6): 377-382.

Peckmann, T. R., K. Orr, S. Meek, and S. K. Manolis 2015b Sex Determination from the Talus in a Contemporary Greek Population Using Discriminant Function Analysis. Journal of Forensic and Legal Medicine 33: $14-19$.

Peckmann, T. R., S. Scott, S. Meek, P. Mahakkanukeauh 2017 Sex Estimation from the Scapula in a Contemporary Thai Population: Applications for Forensic Anthropology. Science and Justice 57: 270-275.

Pelin, C., I. Duyar, E. M. Kayahan, R. Zağyapan, A. M. Ağildere, and A. Erar 2005 Body Height Estimation Based on Dimensions of Sacral and Coccygeal Vertebrae. Journal of Forensic Sciences 50(2): 294-7.

Pelin, C., R. Zağyapan, C. Yazıc1, and A. Kürkçüoğlu 2010 Body Height Estimation from Head and Face Dimensions: A Different Method. Journal of Forensic Sciences 55(5): 1326-1330.

Perola, M., S. Sammalisto, T. Hiekkalinna, N. G. Martin, P. M. Visscher, G. W. Montgomery, J. R. Harris, D. Boomsma, G. Willemsen, J. J. Hottenga, K. Christensen, K. O. Kyvik, T. I. A. Sørensen, N. L. Pedersen, P. K. E. Magnusson, T. D. Spector, E. Widen, K. Silventoinen, J. Kaprio, A. Palotie, L. Peltonen, and GenomEUtwin Project 2007 Combined Genome Scans for Body Stature in 6,602 European Twins: Evidence for Common Caucasian Loci. PLOS Genetics 3(6): 1019.e97-1028.e97.

Pfeiffer, S., J. Heinrich, A. Beresheim, and M. Alblas 2016 Cortical Bone Histomorphology of Known-Age Skeletons from the Kirsten Collection, Stellenbosch University, South Africa. American Journal of Physical Anthropology 160(1): 137-147.

Phenice, T. W. 1969 A Newly Developed Visual Method of Sexing the Os Pubis. American Journal of Physical Anthropology 30(2): 297-301.

Piazza, S. J.

2005 Mechanics of the Subtalar Joint and Its Function During Walking. Foot and Ankle Clinics of North America 10(3): 425-442. 
Pickering, R. B.

1986 Population Differences in the Calcaneus as Determined by Discriminant Function Analysis. In Forensic Osteology: Advances in the Identification of Human Remains. K.J. Reichs, ed. Pp. 160-170. Springfield: Charles C. Thomas Ltd.

Pininski, M., and D. Brits 2014 Estimating Stature in South African Populations Using Various Measures of the Sacrum. Forensic Science International 234: 182.e1-182.e7.

Pongchaiyakul, C., S. Charoenkiatkul, V. Kosulwat, N. Rojroongwasinkul, and R. Rajatanavin 2008 Dietary Calcium Intake among Rural Thais in Northeastern Thailand. Journal of the Medical Association of Thailand 91(2): 153-158.

Pongou, R., M. Ezzati, and J. A. Salomon 2006 Household and Community Socioeconomic and Environmental Determinants of Child Nutritional Status in Cameroon. BMC Public Health 6(98): 1-19.

Prasada Rao, P. D. 1966 Squatting Facets on the Talus and Tibia in Australian Aborgines. Archaeology and Physical Anthropology in Oceania 1(1): 51-56.

Preece, M. A.

1996 The Genetic Contribution to Stature. Hormone Research in Paediatrics 45(2): 56-58.

Prins, H. E. L. 2002 The Mikmaq: Resistance, Accommodation, and Cultural Survival. Case Studies in Cultural Anthropology. Mason, Ohio: Cengage Learning.

Prinz, M., A. Carracedo, W. R. Mayr, et al. 2007 DNA Commission of the International Society for Forensic Genetics (ISFG): Recommendations Regarding the Role of Forensic Genetics for Disaster Victim Identification (DVI). Forensic Science International: Genetics 1(1): 3-12.

Pryor, J. W.

1923 Differences in the Time of Development of Centers of Ossification in the Male and Female Skeleton. The Anatomical Record 25(5): 257-273.

Rajesh, R.

2014 Determination of Stature and Sex from Human Clavicle. International Journal of Recent Trends in Science and Technology 10: 111-112. 
Ranabir, S., and K. Reetu

2011 Stress and Hormones. Indian Journal of Endocrinology and Metabolism 15(1): 18 .

Rani, Y., S.K. Naik, A.K. Singh, and Murari, A.

2011 Correlation of Stature of Adult with the Length of Clavicle. Indian Journal of Forensic and Medicine and Pathology 33(3): 194-196.

Rappold, G., W. F. Blum, E. P. Shavrikova, B. J. Crowe, R. Roeth, C. A. Quigley, J. L. Ross, and B. Niesler

2007 Genotypes and Phenotypes in Children with Short Stature: Clinical Indicators of SHOX Haploinsufficiency. Journal of Medical Genetics 44(5): 306-313.

Raxter, M. H., B. M. Auerbach, and C. B. Ruff

2006 Revision of the Fully Technique for Estimating Statures. American Journal of Physical Anthropology 130(3): 374-384.

Reagan, P. B., P. J. Salsberry, M. Z. Fang, W. P. Gardner, and K. Pajer 2012 African-American/White Differences in the Age of Menarche: Accounting for the Difference. Social Science \& Medicine (1982) 75(7): 1263-1270.

Regina v. Mohan, 2 S.C.R. 9 File No. 23063, 1994. Accessed June 19, 2017.

Reis, M., L. Ramiro, E. Godeau, M. Gaspar de Matos, and A. Social 2016 Age at Menarche in Portuguese Adolescents: A Cross-Sectional Study with HBSC Portuguese Survey. Global Advanced Research Journal of Educational Research and Review 5(4): 55-62.

Richard, S. A., R. E. Black, R. H. Gilman, R. L. Guerrant, G. Kang, C. F. Lanata, K. Mølbak, Z. A. Ramussen, R. B. Sack, P. Valentiner-Branth, and W. Checkley 2013 Diarrhea in Early Childhood: Short-Term Association with Weight and Long-Term Association with Length. American Journal of Epidemiology 178(7): 1129-1138.

Rickland, D. E., and P. V. Tobias 1986 Unusually Low Sexual Dimorphism of Endocranial Capacity in a Zulu Cranial Series. American Journal of Physical Anthropology 71: 285-293.

Riepert, T., T. Drechsler, H. Schild, B. Nafe, and R. Mattern 1996 Estimation of Sex on the Basis of Radiographs of the Calcaneus. Forensic Science International 77(3): 133-140.

Risman, B. J. 2004 Gender as a Social Structure: Theory Wrestling with Activism. Gender and Society 18(4): 429-450. 
Roche, A. F.

1979 Secular Trends in Human Growth, Maturation, and Development.

Monographs of the Society for Research in Child Development 44(3-4): 1-120.

Rodhe, $\mathrm{H}$.

1990 A Comparison of the Contribution of Various Gases to the Greenhouse Effect. Science 248(4960): 1217-1219.

Rodríguez, S., M. S. Rodríguez-Calvo, A. González, M. Febrero-Bande, and J. I. MuñozBarús

2016 Estimating Height from the First and Second Cervical Vertebrae in a Spanish Population. Legal Medicine 19: 88-92.

Rodríguez, S., X. Miguéns, M. S. Rodríguez-Calvo, M. Febrero-Bande, and J. I. MuñozBarús

2012 Estimating Adult Stature from Radiographically Determined Metatarsal

Length in a Spanish Population. Forensic Science International 226(1-3): 297.e1297.e4.

Rogers, T., and S. Saunders

1994 Accuracy of Sex Determination Using Morphological Traits of the Human Pelvis. Journal of Forensic Sciences 39(4): 1047-56.

Rojroongwasinkul, N, K. Kijboonchoo, W. Wimonpeerapattana, S. Purttiponthanee, U. Yamborisut, A. Boonpraderm, P. Kunapan, W. Thasanasuwan, and I. Khouw 2013 SEANUTS: The Nutritional Status and Dietary Intakes of 0.5-12-Year-Old Thai Children. The British Journal of Nutrition 110(3): S36-44.

Ruff, C. B.

1987 Sexual Dimorphism in Human Lower Limb Bone Structure: Relationship to Subsistence Strategy and Sexual Division of Labor. Journal of Human Evolution 16(5): 391-416.

Ruff, C. B.

2008 Biomechanical Analyses of Archaeological Human Skeletons. In M.A.

Katzenberg, S.R. Saunders, eds. Biological Anthropology of the Human Skeleton. $2^{\text {nd }}$ edition. Pp. 183-206. Hoboken: Wiley-Liss.

Ruff, C. B., B. M. Holt, M. Niskanen, V. Sladék, M. Berner, E. Garofalo, H. M. Garvin, M. Hora, H. Maijanen, S. Niinimäki, K. Salo, E. Schuplerová, and D. Tompkins 2012 Stature and Body Mass Estimation from Skeletal Remains in the European Holocene. American Journal of Physical Anthropology 148(4): 601-617.

Ruiz, J. R., R. A. Christman, and H. J. Hillstrom 1993 Anatomical Considerations of the Peroneal Tubercle. Journal of the American Podiatric Medical Association 83(10): 563-575. 
Ryan, I., and M. A. Bidmos

2007 Skeletal Height Reconstruction from Measurements of the Skull in

Indigenous South Africans. Forensic Science International 167(1): 16-21.

S. A. Montzka, E. J. Dlugokencky, and J. H. Butler

2011 Non-CO2 Greenhouse Gases and Climate Change. Nature 476(7358): $43-$ 50.

Saadeh, F. A., A. H. Faud, S. M. Mohmaud, and E. E. Marwan 2000 Patterns of Talar Articular Facets of Egyptian Calcanei. Journal of the Anatomical Society of India 49(1): 6-8.

Salathé, E. P., G. A. Arangio, and E. P. Salathé 1986 A Biomechanical Model of the Foot. Journal of Biomechanics 19(12): 9891001.

Sangvichien, S. J., T. Pomipak, and B. Kalapravitt 1950 Sex Differentiation by Preauricular Sulcus. Special Issue 60 Years Anniversary of Faculty of Medicine and Siriraj Hospital: Siriraj Medical Journal: 9-19.

Sankas, S. H. 1930 Relation of Cranial Module to Capacity. American Journal of Physical Anthropology 14(2): 305-315.

Sanna, S., A. U. Jackson, R. Nagaraja, et al. 2008 Common Variants in the GDF5-UQCC Region Are Associated with Variation in Human Height. Nature Genetics 40(2): 198-203.

Sarrafian, S. K.

1993 Anatomy of the Foot and Ankle: Descriptive, Topographic, Functional. 2nd ed. Philadelphia: J.B. Lippincott.

Satinoff, M. I. 1972 Study of the Squatting Facets of the Talus and Tibia in Ancient Egyptians. Journal of Human Evolution 1(2): 209-212.

Sauer, N. J.

1992 Forensic Anthropology and the Concept of Race: If Races Don't Exist, Why Are Forensic Anthropologists So Good at Identifying Them? Social Science \& Medicine 34(2): 107-111. 
Saul, J. M., and F. P. Saul 2005 "The Game Is Afoot! Feet Help Solve Forensic Puzzles in the United States and Overseas" In: Forensic Medicine of the Lower Extremity: Human Identification and Trauma Analyis of the Thigh, Leg, and Foot. J. Rich, D. E. Dean, R. H. Powers, eds. Pp. 359-374. New Jersey: Humana Press.

Scheuer, L. 2000 Developmental Juvenile Osteology. San Diego, California: Academic Press.

Scheuer, L., and S. Black 2004 The Juvenile Skeleton. San Diego, California: Academic Press.

Schneider, M., R. Norman, C. Parry, D. Bradshaw, A. Plüddemann, and the South African Comparative Risk Assessment Collaborating Group 2007 Estimating the Burden of Disease Attributable to Alcohol Use in South Africa in 2000. South African Medical Journal 97(8 Pt 2): 664-672.

Scott, S., S. Ruengdit, T. R. Peckmann, and P. Mahakkanukrauh 2017 Sex Estimation from Measurements of the Calcaneus: Applications for Personal Identification in Thailand. Forensic Science International 278: 405.e1405.e8.

Seekings, J. 2008 The Continuing Salience of Race: Discrimination and Diversity in South Africa. Journal of Contemporary African Studies 26(1): 1-25.

Sharada, R., K. Sneha, C. Gupta, S. Pai, and G. Rairam 2012 Non-Metrical Study of the Pattern of Talar Articular Facets in South Indian Dry Calcanei. Surgical and Radiologic Anatomy 34(6): 487-491.

Shibata, Y., E. Sakuma, Y. Yoshida, K. Wakabayashi, H. Iguchi, I. Sekiya, T. Otsuka, and I. Wada 2014 Morphometric Analysis of the Peroneal Tubercle Using a ThreeDimensional Computed Tomography Model. The Foot 24(4): 200-202.

Shintaku, K., and Y. Furuya 1990 Estimation of Stature Based on the Proximal Phalangeal Length of Japanese Women's Hands. Journal of UOEH (University of Occupational and Environmental Health) 12(2): 215-9.

Shrestha, R., P. K. Shrestha, H. Wasti, T. Kadel, T. Kanchan, and K. Krishan 2015 Craniometric Analysis for Estimation of Stature in Nepalese Population-A Study on an Autopsy Sample. Forensic Science International 248: 187.e1-187.e6. 
Shulin, P., and Fangwu, Z.

1983 Estimation of Stature from Skull, Clavicle, Scapula and Os Coxa of Male Adult of Southern Chinese. Acta Anthropologica Sinica 2: 253-259

Silva, A. M.

1995 Sex Assessment Using the Calcaneus and Talus. Anthropologia Portuguesa 13: $107-119$.

Silventoinen, K., J. Kaprio, and E. Lahelma 2000 Genetic and Environmental Contributions to the Association between Body Height and Educational Attainment: A Study of Adult Finnish Twins. Behavior Genetics 30(6): 477-485.

Silventoinen, K., J. Kaprio, E. Lahelma, R. J. Viken, and R. J. Rose 2001 Sex Differences in Genetic and Environmental Factors Contributing to Body-Height. Twin Research: The Official Journal of the International Society for Twin Studies 4(1): 25-29.

Simmons, T., R. L. Jantz, and W. M. Bass 1990 Stature Estimation from Fragmentary Femora: A Revision of the Steele Method. Journal of Forensic Sciences 35(3): 628-36.

Singh, B., and H. S. Sohal 1952 Estimation of Stature from Clavicle in Punjabis; a Preliminary Report. The Indian Journal of Medical Research 40(1): 67-71.

Singh, I.

1959 Squatting Facets on the Talus and Tibia in Indians. Journal of Anatomy 93(Pt.4): 540-550.

Singh, J., R. K. Pathak, and K. H. Chavali

2011 Skeletal Height Estimation from Regression Analysis of Sternal Lengths in a Northwest Indian Population of Chandigarh Region: A Postmortem Study. Forensic Science International 206(1): 211.e1-211.e8.

Smay, D., and G. Armelagos 2000 Galileo Wept: A Critical Assessment of the Use of Race in Forensic Anthropology. Transforming Anthropology 9(2): 19-29.

Smedley, A.

1998 "Race" and the Construction of Human Identity. American Anthropologist 100(3): 690-702.

Smedley, A., and B. D. Smedley 2005 Race as Biology Is Fiction, Racism as a Social Problem Is Real. American Psychologist 60(1): 16-26. 
Smith, L. C., M. T. Ruel, and A. Ndiaye 2005 Why Is Child Malnutrition Lower in Urban Than in Rural Areas? Evidence from 36 Developing Countries. World Development 33(8): 1285-1305.

Smith, R. J., G. H. Albrecht, J. Damuth, M. Di Bacco, M. Fortelius, P. D. Gingerich, L. R. Godfrey, M. R. Sutherland, W. L. Jungers, S. R. Leigh, M. D. Leney, R. A. Foley, W. R. Leonard, M. L. Robertson, W. Leutenegger, H. M. McHenry, R. D. Martin, D. Pilbeam, J. M. Plavcan, P. E. Wheeler, B. A. Wood, and M. Collard 1996 Biology and Body Size in Human Evolution: Statistical Inference Misapplied [and Comments and Reply]. Current Anthropology 37(3): 451-481.

Sokal, R. R., and F. J. Rohlf

1995 Biometry. $3^{\text {rd }}$ edition. New York: Freeman and Company.

Solomon, S., J. S. Daniel, T. J. Sanford, D. M. Murphy, G. K. Plattner, R. Knutti, and P. Friedlingstein 2010 Persistence of Climate Changes Due to a Range of Greenhouse Gases. Proceedings of The National Academy of Sciences of The United States of America 107(43): 18354-18359.

Soranzo, N., F. Rivadeneira, U. Chinappen-Horsley, I. Malkina, J. B. Richards, N. Hammond, L. Stolk, A. Nica, M. Inouye, A. Hofman, J. Stephens, E. Wheeler, P. Arp, R. Gwilliam, P. M. Jhamai, S. Potter, A. Chaney, M. J. R. Ghori, R. Ravindrarajah, S. Ermakov, K. Estrada, H. A. P. Pols, F. M. Williams, W. L. McArdle, J. B. van Meurs, R. J. F. Loos, E. T. Dermitzakis, K. R. Ahmadi, D. J. Hart, W. H. Ouwehand, N. J. Wareham, I. Barroso, M. S. Sandhu, D. P. Strachan, G. Livshits, T. D. Spector, A. G. Uitterlinden, and P. Deloukas 2009 Meta-Analysis of Genome-Wide Scans for Human Adult Stature Identifies Novel Loci and Associations with Measures of Skeletal Frame Size. PLoS Genetics 5(4): e1000445, 1-13.

Soren, A.

1993 Arthritis and Related Affections: Clinic, Pathology, and Treatment. Berlin; New York: Spring-Verlag.

Spoor, F., N. Jeffery, and F. Zonneveld 2000 Imaging Skeletal Growth and Evolution. In P. Higgins, M. Cohn, eds. Development Growth and Evolution: Implication for the Study of the Hominid Skeleton. Pp. 123-161. London: Academic Press.

Spradley, M. K. 2006 Biological Anthropological Aspects of the African Diaspora; Geographic Origins, Secular Trends, and Plastic Versus Genetic Influences Utilizing Craniometric Data. Ph.D. dissertation, University of Tennessee. 
Spradley, M. K., and R. L. Jantz 2011 Sex Estimation in Forensic Anthropology: Skull Versus Postcranial Elements. Journal of Forensic Sciences 56(2): 289-296.

Sribanditmongkol, P., P. Pongpanitanont, N. Porntrakulseree, M. Petju, S. Kunaratanapruk, P. Kitkailass, P. Ganjanarintr, and N. Somboonsub Forensic Aspect of Disaster Casualty Management Tsunami Victim Identification in Thailand, World Health Organisation. http://www.who.int/hac/events/tsunamiconf/presentations/ 2_16_forensic_pongruk_doc.pdf, accessed February 27, 2017.

Stavrou, I., C. Zois, J. P. A. Ioannidis, and A. Tsatsoulis 2002 Association of Polymorphisms of the Oestrogen Receptor $\alpha$ Gene with the Age of Menarche. Human Reproduction 17(4): 1101-1105.

Steckel, R.H. 2008 Biological Measures of the Standard of Living. Journal of Economic Perspectives 22(1): 129-152.

Steckley, J., and G. Kirby Letts 2013 Elements of Sociology: A Critical Canadian Introduction. $3^{\text {rd }}$ edition. Don Mills: Oxford University Press.

Steele, D. G. 1970 Estimation of Stature from Fragments of Long Limb Bones. In Personal Identification in Mass Disasters. T. D. Stewart, ed. Pp. 85-97. Washington: Smithsonian, National Museum of Natural History.

Steele, D. G.

1976 The Estimation of Sex on the Basis of the Talus and Calcaneus. American Journal of Physical Anthropology 45: 581-588.

Steele, D. G., and T. W. Mckern 1969 A Method for Assessment of Maximum Long Bone Length and Living Stature from Fragmentary Long Bones. American Journal of Physical Anthropology 31(2): 215-227.

Stewart, T. D. 1979 Essentials of Forensic Anthropology. Springfield, Illinois: Charles C Thomas.

Steyn, M., and M. L. Patriquin 2009 Osteometric Sex Determination from the Pelvis-Does Population Specificity Matter? Forensic Science International 191(1): 113.e1-113.e5. 
Steyn, M., and M. Y. İşcan 1997 Sex Determination from the Femur and Tibia in South African Whites. Forensic Science International 90(1-2): 111-119.

Stini, W. A.

1969 Nutritional Stress and Growth: Sex Difference in Adaptive Response. American Journal of Physical Anthropology 31(3): 417-426.

Stini, W.A.

1972 Reduced Sexual Dimorphism in Upper Arm Muscle Circumference Associated with Protein-Deficient Diet in a South American Population. American Journal of Physical Anthropology 36(3): 341-351.

Stinson, $\mathrm{S}$.

1985 Sex Differences in Environmental Sensitivity during Growth and Development. American Journal of Physical Anthropology 28(S6): 123-147.

Stock, J. T., and A. A. Macintosh 2016 Lower Limb Biomechanics and Habitual Mobility among Mid-Holocene Populations of the Cis-Baikal. Quaternary International 405(Part B). Bioarchaeology of NE Asia: 200-209.

Straus, W. L.

1927 Growth of the Human Foot and It's Evolutionary Significance. Contributions to Embryology 19 (101): 93-134.

Stupar, D.

2007 The Nutrition Transition and the Right to Adequate Food for Adolescents in South Africa. Master's Thesis, University of Oslo.

Sue, J. J.

1755 Sur les Proportions des Squelette de Homme, Examiné Depuis l'âge de Plus Tendre, Jusqu'a B Celui de Vingt Cinq, Soixante Ans, \& Audel. Académie des Sciences 2: 572-585.

Suliman, S., S. Seedat, D. R. Williams, and D. J. Stein 2010 Predictors of Transitions Across Stages of Alcohol Use and Alcohol-Use Disorders in South Africa. Journal of Studies on Alcohol and Drugs 71(5): 695703.

Susman, R. L. 1983 Evolution of the Human Foot: Evidence from Plio-Pleistocene Hominids. Foot \& Ankle 3(6): 365-76. 
Tai, C.C., and A. Williams

2007 Ligaments and Tendons. In Basic Orthopaedic Sciences: The Stanmore Guide. M. Ramachandran, ed. Pp. 71-79. London: Edward Arnold.

Talbot, N. B., E. H. Sobel, B. S. Burke, E. Lindemann, and S. B. Kaufman 1947 Dwarfism in healthy children: its possible relation to emotional, nutritional and endocrine disturbances. New England Journal of Medicine 236: 783-793.

Terazawa, K., H. Akabane, H. Gotouda, K. Mizukami, N. Nagao, and T. Takatori 1990 Estimating Stature from the Length of the Lumbar Part of the Spine in Japanese. Medicine, Science, and the Law 30(4): 354-7.

Thavorntam, W., and N. Tantemsapya 2013 Vegetation Greenness Modeling in Response to Climate Change for Northeast Thailand. Journal of Geographical Sciences 23(6): 1052-1068.

The National Statistics Office 2008 Tobacco and Alcohol Consumption Behaviours Among Thai People in the Year 2007. Bangkok, Thailand: The National Statistics Office.

Thomson, A. 1889 The Influence of Posture on the Form of the Articular Surfaces of the Tibia and Astragalus in the Different Races of Man and the Higher Apes. Journal of Anatomy and Physiology 23(Pt 4): 616-39.

Thomas, A.

1890 Additional Note on the Influence of Posture on the Form of the Articular Surfaces of the Tibia and Astragalus in the Different Races of Man and the Higher Apes. Journal of Anatomy and Physiology 24(Pt 2): 210-7.

Tibbetts, G. L.

1981 Estimation of Stature from the Vertebral Column in American Blacks. Journal of Forensic Sciences 26(4): 715-23.

Tönjes, A., M. Koriath, D. Schleinitz, K. Dietrich, Y. Böttcher, N. W. Rayner, P. Almgren, B. Enigk, O. Richter, S. Rohm, A. Fischer-Rosinsky, A. Pfeiffer, K. Hoffmann, K. Krohn, G. Aust, J. Spranger, L. Groop, M. Blüher, P. Kovacs, M. Stumvoll 2009 Genetic Variation in GPR133 Is Associated with Height: Genome Wide Association Study in the Self-Contained Population of Sorbs. Human Molecular Genetics 18(23): 4662-4668. 
Torimitsu, S., Y. Makino, H. Saitoh, A. Sakuma, N. Ishii, D. Yajima, G. Inokuchi, A. Motomura, F. Chiba, R. Yamaguchi, M. Hashimoto, Y. Hoshioka, and H. Iwase 2016 Stature Estimation from Skull Measurements Using Multidetector Computed Tomographic Images: A Japanese Forensic Sample. Legal Medicine 18: $75-80$.

Torimitsu, S., Y. Makino, H. Saitoh, A. Sakuma, N. Ishii, D. Yajima, G. Inokuchi, A. Motomura, F. Chiba, R. Yamaguchi, M. Hashimoto, Y. Hoshioka, and H. Iwase 2017 Stature Estimation in a Contemporary Japanese Population Based on Clavicular Measurements Using Multidetector Computed Tomography. Forensic Science International 275: 316.e1-316.e6.

Torimitsu, S., Y. Makino, H. Saitoh, A. Sakuma, N. Ishii, M. Hayakawa, G. Inokuchi, A. Motomura, F. Chiba, Y. Hoshioka, and H. Iwase 2015 Stature Estimation in Japanese Cadavers Based on Scapular Measurements Using Multidetector Computed Tomography. International Journal of Legal Medicine 129(1): 211-218.

Torimitsu, S., Y. Makino, H. Saitoh, N. Ishii, M. Hayakawa, D. Yajima, G. Inokuchi, A. Motomura, F. Chiba, and H. Iwase 2014 Stature Estimation in Japanese Cadavers Using the Sacral and Coccygeal Length Measured with Multidetector Computed Tomography. Legal Medicine 16(1): 14-19.

Tortora, G. J., and B. H. Derrickson 2014 Principles of Anatomy and Physiology, $14^{\text {th }}$ edition. New York: Wiley.

Traithepchanapai, P., P. Mahakkanukrauh, and E. F. Kranioti 2016 History, Research and Practice of Forensic Anthropology in Thailand. Forensic Science International 261: 167.e1-167.e6.

Trinkaus, E.

1975 Squatting among the Neandertals: A Problem in the Behavioral Interpretation of Skeletal Morphology. Journal of Archaeological Science 2(4): 327-351.

Troesken, W. 2004 Water, Race, and Disease. Cambridge: MIT Press.

Trotter, $\mathrm{M}$.

1970 Estimation of Stature from Intact Limb Bones. In Personal Identification in Mass Disasters. T. D. Stewart, ed. Pp. 71-84. Washington: Smithsonian, National Museum of Natural History.

Trotter, M., and G. C. Gleser 1952 Estimation of Stature from Long Bones of American Whites and Negroes. American Journal of Physical Anthropology 10(4): 463-514. 
Trotter, M., and G. C. Gleser

1958 A Re-Evaluation of Estimation of Stature Based on Measurements of Stature Taken during Life and of Long Bones after Death. American Journal of Physical Anthropology 16(1): 79-123.

Trussell, J., and R. Steckel

1978 The Age of Slaves at Menarche and Their First Birth. The Journal of Interdisciplinary History 8(3): 477-505.

Turok, I., and J. Borel-Saladin 2016 Backyard Shacks, Informality and the Urban Housing Crisis in South Africa: Stopgap or Prototype Solution? Housing Studies 31(4): 384-409.

Turton, R. W., and B. E. Chalmers 1990 Apartheid, Stress and Illness: The Demographic Context of Distress Reported by South African Africans. Social Science \& Medicine 31(11): 11911200.

Van Aalst, M. K.

2006 The Impacts of Climate Change on the Risk of Natural Disasters. Disasters 30(1): 5-18.

van der Berg, S., R. Burger, R. Burger, M. Louw, and D. Yu 2006 Trends in Poverty and Inequality Since the Political Transition. SSRN Scholarly Paper, ID 982093. Rochester, New York: Social Science Research Network.

van Wyk, S.

1968 Die Afrikaner in die Beroepslewe van die Stad. Pretoria: Academica.

Viladot, A., J. C. Lorenzo, J. Salazar, and A. Rodríguez

1984 The Subtalar Joint: Embryology and Morphology. Foot \& Ankle 5(2): 5466.

Wang, Y., B. He, S. Herath, S. Basnayake, and W. Huang 2014 Climate Change Scenarios Analysis in Coastal Region of Thailand. Journal of Coastal Research: 160-167.

Wankhede, K. P., V. P. Anjankar, M. P. Parchand, N. Y. Kamdi, and S. T. Patil 2015 Estimation of Stature from Head Length and Head Breadth in Central Indian Population: An Anthropometric Study. International Journal of Anatomy and Research 3(1): 954-957.

Webster, P. J., G. J. Holland, J. A. Curry, and H. R. Chang 2005 Changes in Tropical Cyclone Number, Duration, and Intensity in a Warming Environment. Science 309(5742): 1844-1846. 
Weedon, M. N., H. Lango, C. M. Lindgren, C. Wallace, D. M. Evans, M. Mangino, R. M. Freathy, J. R. Perry, S. Stevens, A. S. Hall, N. J. Samani, B. Shields, I. Prokopenko, M. Farrall, A. Dominiczak, Diabetes Genetics Initiative, Welcome Trust Case Control Consortium, T. Johnson, S. Bergmann, J. S. Beckmann, P. Vollenweider, D. M. Waterworth, V. Mooser, C. N. Palmer, A. D. Morris, W. H. Ouwehand, Cambridge GEM Consortium, J. H. Zhao, S. Li, R. J. Loos, I. Barroso, P. Deloukas, M. S. Sandhu, E. Wheeler, N. Soranzo, M. Inouye, N. J. Wareham, M. Caulfield, P. B. Munroe, A. T. Hattersley, M. I. McCarthy, and T. M. Frayling 2008 Genome-Wide Association Analysis Identifies 20 Loci That Influence Adult Height. Nature Genetics 40(5): 575-583.

Weedon, M. N., G. Lettre, R. M. Freathy, C. M. Lindgren, B. F. Voight, J. R. Perry, K. S. Elliott, R. Hackett, C. Guiducci, B. Shields, E. Zeggini, H. Lango, V. Lyssenko, N. J. Timpson, N. P. Burtt, N. W. Rayner, R. Saxena, K. Ardlie, J. H. Tobias, A. R. Ness, S. M. Ring, C. N. Palmer, A. D. Morris, L. Peltonen, V.

Salomaa, Diabetes Genetics Initiative, Welcome Trust Case Control Consortium, G. Davey Smith, L. C. Groop, A. T. Hattersley, M. I. McCarthy, J. N. Hirschhorn, and T. M. Frayling 2007 A Common Variant of HMGA2 Is Associated with Adult and Childhood Height in the General Population. Nature Genetics 39(10): 1245-1250.

Wilbur, A. K.

1998 The Utility of Hand and Foot Bones for the Determination of Sex and the Estimation of Stature in a Prehistoric Population from West-Central Illinois. International Journal of Osteoarchaeology 8(3): 180-191.

Williams, B. A., and T. L. Rogers 2006 Evaluating the Accuracy and Precision of Cranial Morphological Traits for Sex Determination. Journal of Forensic Sciences 51(4): 729-735.

Wilson, R. J., N. P. Herrmann, and L. Meadows Jantz 2010 Evaluation of Stature Estimation from the Database for Forensic Anthropology* $\dagger$. Journal of Forensic Sciences 55(3): 684-689.

Winant, $\mathrm{H}$. 2000 Race and Race Theory. Annual Review of Sociology 26: 169-185.

Witherspoon, D. J., S. Wooding, A. R. Rogers, E. E. Marchani, W. S. Watkins, M. A. Batzer, and L. B. Jorde 2007 Genetic Similarities within and between Human Populations. Genetics 176(1): 351-9.

World Bank. 2017. “China” Accessed October 2, 2017. https://data.worldbank.org/country/china?view=chart 
World Bank. 2017. "Colombia" Accessed October 2, 2017. https://data.worldbank.org/country/colombia?view=chart

World Bank. 2017. "Greece” Accessed October 2, 2017. https://data.worldbank.org/country/greece?view=chart

World Bank. 2017. "Germany” Accessed October 2, 2017. https://data.worldbank.org/country/germany?view=chart

World Bank. 2017. "Italy" Accessed October 2, 2017. https://data.worldbank.org/country/italy?view=chart

World Bank. 2017. "South Africa" Accessed October 2, 2017. https://data.worldbank.org/country/south-africa?view=chart

World Bank. 2017. “Turkey” Accessed October 2, 2017. https://data.worldbank.org/country/turkey?view=chart

World Bank. 2017. "World Bank in Thailand: Overview” Accessed October 2, 2017. http://www.worldbank.org/en/country/thailand/overview

World Health Organization (WHO) 2005 "Child Malnutrition" Accessed October 10, 2017. http://www.who.int/countries/zaf/en/

Wright, K., A. Mundorff, J. Chaseling, A. Forrest, C. Maguire, and D. I. Crane 2015 A New Disaster Victim Identification Management Strategy Targeting "near Identification-Threshold" Cases: Experiences from the Boxing Day Tsunami. Forensic Science International 250: 91-97.

Xing, J., W. S. Watkins, A. Shlien, E. Walker, C. D. Huff, D. J. Witherspoon, Y. Zhang, T. S. Simonson, R. B. Weiss, J. D. Schiffman, D. Malkin, S. R. Woodward, and L. B. Jorde 2010 Toward a More Uniform Sampling of Human Genetic Diversity: A Survey of Worldwide Populations by High-Density Genotyping. Genomics 96(4): 199210.

Yao, Y.G., L. Nie, H. Harpending, Y. X. Fu, Z. G. Yuan, and Y. P. Zhang 2002 Genetic Relationship of Chinese Ethnic Populations Revealed by MtDNA Sequence Diversity. American Journal of Physical Anthropology 118(1): 63-76.

Yonguc, G., A. Kurtulus, O. Bayazit, E. Adiguzel, I. Unal, S. Demir, and K. Acar 2015 Estimation of Stature and Sex from Sternal Lengths: An Autopsy Study. Anatomical Science International 90(2): 89-96. 
Zaher, J. F., N. F. M. El-Ameen, and A. E. Seedhom

2011 Stature Estimation Using Anthropometric Measurements from Computed Tomography of Metacarpal Bones among Egyptian Population. Egyptian Journal of Forensic Sciences 1(2): 103-108.

Zakaria, M. S., A. H. Mohammed, S. R. Habib, M. M. Hanna, and A. L. Fahiem 2010 Calcaneus Radiograph as a Diagnostic Tool for Sexual Dimorphism in Egyptians. Journal of Forensic and Legal Medicine 17(7): 378-382.

Zhang, K., J. H. Cui, Y. Z. Luo, F. Fan, M. Yang, X. H. Li, W. Zhang, and Z. H. Deng 2016 Estimation of Stature and Sex from Scapular Measurements by ThreeDimensional Volume-Rendering Technique Using in Chinese. Legal Medicine 21: 58-63.

Zhang, K., Y.Z. Luo, F. Fan, J. Q. Zheng, M. Yang, T. Li, T. Pang, J. Zhang, and Z. H. Deng 2015 Stature Estimation from Sternum Length Using Computed Tomographyvolume Rendering Technique Images of Western Chinese. Journal of Forensic and Legal Medicine 35: 40-44.

Zhao, J., M. Li, J. P. Bradfield, H. Zhang, F. D. Mentch, K. Wang, P. M. Sleiman, C. E. Kim, J. T. Glessner, C. Hou, B. J. Keating, K. A. Thomas, M. L. Garris, S. Deliard, E. E. C. Frackelton, F. G. Otieno, R. M. Chiavacci, R. I. Berkowitz, H. Hakonarson, and S. F. A. Grant 2010 The Role of Height-Associated Loci Identified in Genome Wide Association Studies in the Determination of Pediatric Stature. BMC Medical Genetics 11: 96. 
APPENDIX A:

\section{CHIANG MAI SKELETAL COLLECTION RAW DATA}




\begin{tabular}{|c|c|c|c|c|c|c|c|c|c|c|c|c|c|c|c|c|c|}
\hline $\begin{array}{l}\text { Catalogue } \\
\text { No. }\end{array}$ & ID & $\begin{array}{l}\text { Date of } \\
\text { Birth }\end{array}$ & $\begin{array}{l}\text { Date of } \\
\text { Death }\end{array}$ & $\begin{array}{l}\text { Age } \\
\text { (years) }\end{array}$ & $\begin{array}{l}\text { Age Group } \\
\text { (years) }\end{array}$ & Sex & $\begin{array}{l}\text { Living Height } \\
\text { (cm) }\end{array}$ & $\begin{array}{l}\text { MAXL } \\
(\mathbf{m m})\end{array}$ & $\begin{array}{l}\text { MAXH } \\
(\mathbf{m m})\end{array}$ & $\begin{array}{l}\text { CFH } \\
(\mathbf{m m})\end{array}$ & $\begin{array}{l}\text { BH } \\
(\mathbf{m m})\end{array}$ & $\begin{array}{l}\text { MINB } \\
(\mathbf{m m})\end{array}$ & $\begin{array}{l}\text { LAL } \\
(\mathbf{m m})\end{array}$ & $\begin{array}{l}\text { MIDB } \\
(\mathbf{m m})\end{array}$ & $\begin{array}{l}\text { DAFB } \\
(\mathbf{m m})\end{array}$ & $\begin{array}{l}\text { DAFL } \\
(\mathbf{m m})\end{array}$ & $\begin{array}{l}\text { MTAL } \\
(\mathbf{m m})\end{array}$ \\
\hline $110 / 57$ & 1 & 1995 & 2014 & 19 & $19-25$ & F & 160 & 74.47 & 41.91 & 23.75 & 35.53 & 23.45 & 47.9 & 36.8 & 26.08 & 28.48 & 55.09 \\
\hline $3 / 50$ & 2 & 1981 & 2007 & 26 & $26-30$ & F & 172 & 79.04 & 45.95 & 24.47 & 41.37 & 21.94 & 45.97 & 38.43 & 29.68 & 27.06 & 57.47 \\
\hline $81 / 54$ & 3 & 1989 & 2011 & 22 & $19-25$ & F & 150 & 66.47 & 39.11 & 23.35 & 36.08 & 22.9 & 42.66 & 36.17 & 25.62 & 25.03 & 47.57 \\
\hline $30 / 55$ & 4 & 1982 & 2012 & 30 & $26-30$ & F & 165 & 80.82 & 41.49 & 24.92 & 35.53 & 26.4 & 45.85 & 38.82 & 24.75 & 25.55 & 53.55 \\
\hline $342 / 56$ & 5 & 1983 & 2013 & 30 & $26-30$ & F & 150 & 65.18 & 35.87 & 22.97 & 31.2 & 22.96 & 43.62 & 37.86 & 24.86 & 24.44 & 49.05 \\
\hline $131 / 50$ & 6 & 1976 & 2007 & 31 & $31-35$ & F & 170 & 78.72 & 42.17 & 26.36 & 39.73 & 26.72 & 49.19 & 41.46 & 27.47 & 27.03 & 55.35 \\
\hline $28 / 56$ & 7 & 1977 & 2013 & 36 & $36-40$ & $\mathrm{~F}$ & 155 & 67.06 & 41.04 & 21.57 & 32.07 & 22.23 & 43.09 & 35.88 & 25.85 & 25.9 & 49.7 \\
\hline $312 / 56$ & 8 & 1976 & 2013 & 37 & $36-40$ & $\mathrm{~F}$ & 158 & 77.03 & 41.04 & 24.99 & 36.74 & 22.33 & 45.32 & 36.83 & 28.5 & 27.03 & 50.39 \\
\hline $122 / 51$ & 9 & 1968 & 2008 & 40 & $36-40$ & F & 150 & 66.76 & 39.51 & 21.55 & 30.09 & 21.59 & 38.16 & 34.77 & 23.49 & 21.68 & 46.44 \\
\hline $157 / 50$ & 10 & 1963 & 2007 & 44 & $41-45$ & F & 155 & 69.7 & 38.95 & 26.72 & 34.48 & 23.94 & 41.31 & 38.33 & 24.39 & 23.59 & 50.86 \\
\hline $50 / 50$ & 11 & 1961 & 2007 & 46 & $46-50$ & F & 165 & 75.33 & 41.78 & 24.85 & 33.94 & 22.62 & 46.15 & 38.46 & 28.32 & 25.5 & 52.35 \\
\hline $61 / 52$ & 12 & 1960 & 2009 & 49 & $46-50$ & F & 150 & 69.36 & 36.02 & 23 & 34.95 & 24.8 & 43.77 & 34.68 & 24.85 & 22.9 & 50.94 \\
\hline $23 / 50$ & 13 & 1957 & 2007 & 50 & $46-50$ & $\mathrm{~F}$ & 158 & 76.88 & 41.45 & 25.16 & 37.72 & 25.36 & 45.13 & 37.9 & 26.51 & 27.25 & 53.88 \\
\hline $108 / 49$ & 14 & 1955 & 2006 & 51 & $51-55$ & F & 167 & 77.57 & 40.34 & 23.62 & 34.59 & 23.55 & 48.2 & 37.63 & 26.53 & 26.76 & 54.1 \\
\hline $163 / 52$ & 15 & 1955 & 2009 & 54 & $51-55$ & F & 148 & 66.78 & 39.03 & 23.3 & 31.5 & 25.35 & 41.52 & 38.37 & 25.52 & 27.04 & 50.47 \\
\hline $119 / 49$ & 16 & 1949 & 2006 & 57 & $56-60$ & $\mathrm{~F}$ & 155 & 81.06 & 40.45 & 26.99 & 36.1 & 26.37 & 48.13 & 39.93 & 30.81 & 26.95 & 54.28 \\
\hline $60 / 52$ & 17 & 1950 & 2009 & 59 & $56-60$ & F & 155 & 73.75 & 44.58 & 24.5 & 38.74 & 23 & 47.1 & 37.61 & 27.33 & 25.79 & 51.02 \\
\hline $120 / 50$ & 18 & 1947 & 2007 & 60 & $56-60$ & F & 150 & 72.01 & 38.24 & 23.6 & 34.38 & 23.67 & 43.28 & 36.52 & 27.41 & 25.57 & 50.7 \\
\hline $90 / 50$ & 19 & 1945 & 2007 & 62 & $61-65$ & F & 150 & 69.53 & 41.52 & 23.68 & 33.41 & 25.29 & 41.58 & 34.18 & 24.26 & 23.82 & 48.84 \\
\hline $1 / 56$ & 20 & 1949 & 2013 & 64 & $61-65$ & F & 158 & 63.81 & 34.67 & 25.3 & 30.8 & 22.54 & 38.2 & 34.51 & 26.53 & 23.88 & 44.33 \\
\hline $166 / 52$ & 21 & 1943 & 2009 & 66 & $66-70$ & F & 150 & 68.5 & 37.2 & 22.37 & 33.49 & 22.65 & 40.97 & 34.75 & 25.67 & 24.79 & 51.49 \\
\hline $130 / 50$ & 22 & 1938 & 2007 & 69 & $66-70$ & F & 163 & 77.63 & 39.29 & 24.67 & 34.29 & 24.68 & 45.92 & 38.92 & 29.39 & 29.52 & 53.41 \\
\hline $17 / 50$ & 23 & 1937 & 2007 & 70 & $66-70$ & F & 153 & 74.36 & 44.76 & 24.85 & 33.48 & 23.32 & 46.06 & 37.55 & 25.91 & 28.33 & 51.37 \\
\hline $19 / 52$ & 24 & 1936 & 2009 & 73 & $71-75$ & F & 145 & 70.95 & 38.24 & 21.96 & 30.58 & 21.46 & 42.7 & 36.15 & 25.15 & 25.83 & 46.62 \\
\hline $1 / 53$ & 25 & 1936 & 2010 & 74 & $71-75$ & $\mathrm{~F}$ & 155 & 74.36 & 50.41 & 27.19 & 35.44 & 21.73 & 46.34 & 38.43 & 28.47 & 26.17 & 52.03 \\
\hline $91 / 51$ & 26 & 1930 & 2008 & 78 & $76-80$ & $\mathrm{~F}$ & 135 & 67.57 & 40.98 & 24.96 & 32.99 & 22.12 & 42.14 & 36.16 & 26.46 & 23.83 & 46.83 \\
\hline
\end{tabular}




\begin{tabular}{|c|c|c|c|c|c|c|c|c|c|c|c|c|c|c|c|c|c|}
\hline $\begin{array}{l}\text { Catalogue } \\
\text { No. }\end{array}$ & ID & $\begin{array}{l}\text { Date of } \\
\text { Birth }\end{array}$ & $\begin{array}{l}\text { Date of } \\
\text { Death }\end{array}$ & $\begin{array}{l}\text { Age } \\
\text { (years) }\end{array}$ & $\begin{array}{l}\text { Age Group } \\
\text { (years) }\end{array}$ & Sex & $\begin{array}{l}\text { Living } \\
\text { Height (cm) }\end{array}$ & $\begin{array}{l}\text { MAXL } \\
(\mathbf{m m})\end{array}$ & $\begin{array}{l}\text { MAXH } \\
(\mathrm{mm})\end{array}$ & $\begin{array}{l}\text { CFH } \\
(\mathbf{m m})\end{array}$ & $\begin{array}{l}\text { BH } \\
(\mathbf{m m})\end{array}$ & $\begin{array}{l}\text { MINB } \\
(\mathrm{mm})\end{array}$ & $\begin{array}{l}\text { LAL } \\
(\mathbf{m m})\end{array}$ & $\begin{array}{l}\text { MIDB } \\
(\mathrm{mm})\end{array}$ & $\begin{array}{l}\text { DAFB } \\
(\mathbf{m m})\end{array}$ & $\begin{array}{l}\text { DAFL } \\
(\mathbf{m m})\end{array}$ & $\begin{array}{l}\text { MTAL } \\
(\mathbf{m m})\end{array}$ \\
\hline $22 / 50$ & 27 & 1921 & 2007 & 86 & $86-90$ & $\mathrm{~F}$ & 150 & 66.54 & 38.04 & 24.87 & 33.16 & 21.79 & 43.3 & 35.83 & 26.09 & 24.51 & 54.22 \\
\hline $14 / 53$ & 28 & 1923 & 2010 & 87 & $86-90$ & $\mathrm{~F}$ & 150 & 75.87 & 43.57 & 28.61 & 32.49 & 26.82 & 44.86 & 39.78 & 28.52 & 26.47 & 53.46 \\
\hline 5408145 & 29 & 1922 & 2011 & 89 & $86-90$ & F & 150 & 70.81 & 38.76 & 21.67 & 34.39 & 21.77 & 39.82 & 34.61 & 24.71 & 24.88 & 50.08 \\
\hline $185 / 52$ & 30 & 1987 & 2009 & 22 & $19-25$ & M & 158 & 71.1 & 40.82 & 27.84 & 35.41 & 25.3 & 45.43 & 40.86 & 31.48 & 28.85 & 51.51 \\
\hline $142 / 57$ & 31 & 1986 & 2014 & 28 & $26-30$ & M & 180 & 78.49 & 44.55 & 27.46 & 38.9 & 24.08 & 49.01 & 42.11 & 31.1 & 30.02 & 55.71 \\
\hline $120 / 53$ & 32 & 1981 & 2010 & 29 & $26-30$ & M & 170 & 83.66 & 46.71 & 29.88 & 41.49 & 24.94 & 48.93 & 44.05 & 32.05 & 29.11 & 57.61 \\
\hline 5310183 & 33 & 1976 & 2010 & 34 & $31-35$ & M & 163 & 76.74 & 40.39 & 29.2 & 36.43 & 25.49 & 46.09 & 37.67 & 29.57 & 27.11 & 50.52 \\
\hline $122 / 50$ & 34 & 1971 & 2007 & 36 & $36-40$ & M & 180 & 87.02 & 48.12 & 29.13 & 40.5 & 26.23 & 50.14 & 45.12 & 30.01 & 29.47 & 59.12 \\
\hline $131 / 52$ & 35 & 1973 & 2009 & 36 & $36-40$ & M & 165 & 78.25 & 44.25 & 25.92 & 34.25 & 28.14 & 49.59 & 39.62 & 31.05 & 31.57 & 53.98 \\
\hline $184 / 53$ & 36 & 1974 & 2010 & 36 & $36-40$ & $\mathrm{M}$ & 190 & 90.87 & 47.52 & 31.86 & 49.14 & 29.75 & 57.35 & 45 & 32.84 & 31.24 & 63.17 \\
\hline $341 / 56$ & 37 & 1975 & 2013 & 38 & $36-40$ & M & 155 & 72.68 & 42.3 & 25.52 & 39.39 & 25.51 & 44.79 & 39.27 & 31.12 & 28.72 & 52.6 \\
\hline $299 / 56$ & 38 & 1973 & 2013 & 40 & $36-40$ & $\mathrm{M}$ & 160 & 77.84 & 44.47 & 24.5 & 36.84 & 23.94 & 47.91 & 38.08 & 30.14 & 29.25 & 53.48 \\
\hline $109 / 53$ & 39 & 1967 & 2010 & 43 & $41-45$ & M & 150 & 73.69 & 38.7 & 25.28 & 32.45 & 25.83 & 43.55 & 40.08 & 26.12 & 26.36 & 51.62 \\
\hline $1 / 50$ & 40 & 1963 & 2007 & 44 & $41-45$ & $\mathrm{M}$ & 156 & 80.81 & 44.5 & 24.89 & 38.02 & 25.21 & 49.06 & 39.23 & 27.51 & 26.43 & 59.92 \\
\hline $95 / 50$ & 41 & 1958 & 2007 & 49 & $46-50$ & M & 165 & 76.57 & 40.41 & 21.69 & 40.13 & 26.27 & 45.12 & 39.91 & 30.81 & 31.4 & 51.1 \\
\hline $35 / 52$ & 42 & 1954 & 2009 & 55 & $51-55$ & $\mathrm{M}$ & 170 & 87.48 & 42.25 & 28.61 & 40.77 & 28.29 & 54.01 & 44.37 & 30.05 & 31.96 & 62.38 \\
\hline $113 / 52$ & 43 & 1953 & 2009 & 56 & $56-60$ & M & 172 & 85.57 & 41.08 & 31.15 & 38.21 & 29.15 & 50.65 & 43.98 & 27.47 & 32.23 & 59.72 \\
\hline $69 / 50$ & 44 & 1950 & 2007 & 57 & $56-60$ & M & 170 & 82.49 & 41.63 & 27.47 & 39.7 & 25.56 & 50.91 & 43.55 & 31.2 & 30.08 & 57.66 \\
\hline $70 / 56$ & 45 & 1955 & 2013 & 58 & $56-60$ & M & 170 & 76.59 & 43.17 & 27.52 & 36.46 & 26.46 & 47.89 & 40.6 & 28.51 & 27.25 & 55.42 \\
\hline $182 / 52$ & 46 & 1950 & 2009 & 59 & $56-60$ & M & 160 & 83.63 & 47.12 & 25.58 & 41.7 & 29.1 & 51.43 & 41.04 & 30.91 & 28.95 & 54.64 \\
\hline $47 / 50$ & 47 & 1946 & 2007 & 61 & $61-65$ & M & 172 & 80.18 & 42.31 & 26.59 & 40.85 & 24.51 & 48.85 & 40.29 & 29.7 & 29.99 & 57.06 \\
\hline $43 / 51$ & 48 & 1946 & 2008 & 62 & $61-65$ & M & 155 & 76.44 & 42.64 & 24.42 & 41.65 & 23.23 & 47.15 & 38.19 & 29.6 & 27.76 & 57.05 \\
\hline $64 / 50$ & 49 & 1943 & 2007 & 64 & $61-65$ & M & 162 & 79.59 & 40.06 & 27.53 & 39.89 & 25.31 & 49.23 & 39.97 & 30.48 & 29.49 & 57.16 \\
\hline $21 / 52$ & 50 & 1943 & 2009 & 66 & $66-70$ & M & 165 & 84.4 & 43.58 & 28.66 & 42.24 & 27.67 & 47.27 & 41.61 & 27.17 & 32.25 & 57 \\
\hline $12 / 50$ & 51 & 1938 & 2007 & 69 & $66-70$ & $\mathrm{M}$ & 160 & 84.6 & 42.83 & 26.25 & 38.78 & 28.47 & 48.45 & 41.96 & 31.03 & 30.97 & 55.92 \\
\hline $17 / 52$ & 52 & 1939 & 2009 & 70 & $66-70$ & $\mathrm{M}$ & 166 & 76.03 & 44.66 & 27.41 & 37.57 & 26.92 & 49.73 & 39.95 & 28.4 & 31.16 & 57.18 \\
\hline
\end{tabular}




\begin{tabular}{|c|c|c|c|c|c|c|c|c|c|c|c|c|c|c|c|c|c|}
\hline $\begin{array}{l}\text { Catalogue } \\
\text { No. }\end{array}$ & ID & $\begin{array}{l}\text { Date of } \\
\text { Birth }\end{array}$ & $\begin{array}{l}\text { Date of } \\
\text { Death }\end{array}$ & $\begin{array}{l}\text { Age } \\
\text { (years) }\end{array}$ & $\begin{array}{l}\text { Age Group } \\
\text { (years) }\end{array}$ & Sex & $\begin{array}{l}\text { Living } \\
\text { Height (cm) }\end{array}$ & $\begin{array}{l}\text { MAXL } \\
(\mathbf{m m})\end{array}$ & $\begin{array}{l}\text { MAXH } \\
(\mathbf{m m})\end{array}$ & $\begin{array}{l}\text { CFH } \\
(\mathbf{m m})\end{array}$ & $\begin{array}{l}\text { BH } \\
(\mathbf{m m})\end{array}$ & $\begin{array}{l}\text { MINB } \\
(\mathrm{mm})\end{array}$ & $\begin{array}{l}\text { LAL } \\
(\mathbf{m m})\end{array}$ & $\begin{array}{l}\text { MIDB } \\
(\mathrm{mm})\end{array}$ & $\begin{array}{l}\text { DAFB } \\
(\mathrm{mm})\end{array}$ & $\begin{array}{l}\text { DAFL } \\
(\mathrm{mm})\end{array}$ & $\begin{array}{l}\text { MTAL } \\
(\mathrm{mm})\end{array}$ \\
\hline $186 / 55$ & 53 & 1936 & 2012 & 76 & $76-80$ & M & 157 & 75.03 & 40.33 & 24.92 & 37.85 & 24.96 & 47.67 & 42.67 & 29.93 & 27.96 & 50.24 \\
\hline $70 / 50$ & 54 & 1928 & 2007 & 79 & $76-80$ & M & 170 & 84.23 & 44.92 & 27.41 & 41.66 & 25.98 & 51 & 42.7 & 27.45 & 28.17 & 59.54 \\
\hline $33 / 50$ & 55 & 1927 & 2007 & 80 & $76-80$ & M & 173 & 83.5 & 49.47 & 29.1 & 39.64 & 26.39 & 48.11 & 41.1 & 30.3 & 30.23 & 56.43 \\
\hline $14 / 50$ & 56 & 1924 & 2007 & 83 & $81-85$ & M & 178 & 81.58 & 43.18 & 21.5 & 40.07 & 26.97 & 48.21 & 40.01 & 29.29 & 28.98 & 51.68 \\
\hline $140 / 57$ & 57 & 1925 & 2014 & 89 & $86-90$ & M & 155 & 74.83 & 39.18 & 25.85 & 36.57 & 22.13 & 47.01 & 36.9 & 27.74 & 28.09 & 52.34 \\
\hline $116 / 53$ & 58 & 1914 & 2010 & 96 & $96-100$ & M & 150 & 78.45 & 40.66 & 26.41 & 36.96 & 23.12 & 49.78 & 41.4 & 29.34 & 29.25 & 56.94 \\
\hline 5405075 & 59 & 1968 & 2011 & 43 & $41-45$ & $\mathrm{~F}$ & 160 & 78.17 & 42.35 & 27.42 & 36.62 & 21.78 & 48.03 & 39.4 & 27.69 & 26.64 & 50.66 \\
\hline 5405067 & 60 & 1968 & 2011 & 43 & $41-45$ & F & 157 & 72.32 & 38.41 & 24.43 & 34.19 & 23.57 & 42.77 & 35.43 & 26.39 & 23.1 & 48.93 \\
\hline 5408150 & 61 & 1966 & 2011 & 45 & $41-45$ & F & 170 & 79.5 & 50.16 & 25.34 & 37.47 & 26.38 & 49.26 & 41.13 & 27.6 & 28.64 & 57.32 \\
\hline $160 / 52$ & 62 & 1963 & 2009 & 46 & $46-50$ & $\mathrm{~F}$ & 165 & 79.39 & 44.7 & 25.37 & 35.14 & 23.17 & 45.86 & 36.5 & 24.01 & 25.81 & 54.68 \\
\hline $153 / 52$ & 63 & 1962 & 2009 & 47 & $46-50$ & $\mathrm{~F}$ & 165 & 79.33 & 38.67 & 26.31 & 33.06 & 23.09 & 46.27 & 37.87 & 28.16 & 25.94 & 48.34 \\
\hline $25 / 52$ & 64 & 1961 & 2009 & 48 & $46-50$ & $\mathrm{~F}$ & 155 & 73.22 & 42.12 & 22.14 & 38.5 & 20.9 & 45.69 & 35.8 & 57.5 & 26.14 & 51.78 \\
\hline $70 / 53$ & 65 & 1961 & 2010 & 49 & $46-50$ & $\mathrm{~F}$ & 140 & 64.27 & 36.13 & 22.9 & 34.99 & 22.6 & 40.88 & 36.16 & 29.62 & 23.28 & 44.19 \\
\hline $224 / 53$ & 66 & 1961 & 2010 & 49 & $46-50$ & $\mathrm{~F}$ & 155 & 74.29 & 43.53 & 27.63 & 38.15 & 22.83 & 43.17 & 34.56 & 27.44 & 24.83 & 48.35 \\
\hline 5305098 & 67 & 1960 & 2010 & 50 & $46-50$ & $\mathrm{~F}$ & 160 & 68.36 & 37.72 & 23.95 & 33.13 & 24.98 & 43.57 & 38.96 & 25.36 & 26.01 & 50.04 \\
\hline $9 / 54$ & 68 & 1961 & 2011 & 50 & $46-50$ & $\mathrm{~F}$ & 160 & 79.62 & 42.37 & 26.11 & 35.53 & 25.47 & 47.43 & 39.74 & 27.83 & 28.49 & 53.69 \\
\hline $138 / 57$ & 69 & 1963 & 2014 & 51 & $51-55$ & $\mathrm{~F}$ & 160 & 71.61 & 38.86 & 24.6 & 35.38 & 23.64 & 44.75 & 37.46 & 28.27 & 25.3 & 53.99 \\
\hline $109 / 57$ & 70 & 1964 & 2014 & 50 & $46-50$ & F & 150 & 67.52 & 37.48 & 23.36 & 34.24 & 20.37 & 43.11 & 35.89 & 23.36 & 25.05 & 49.08 \\
\hline $95 / 51$ & 71 & 1956 & 2008 & 52 & $51-55$ & $\mathrm{~F}$ & 150 & 68.72 & 39.91 & 24.51 & 31.38 & 23.96 & 42.23 & 34.75 & 25.55 & 24.51 & 47.1 \\
\hline $6 / 52$ & 72 & 1954 & 2009 & 55 & $51-55$ & F & 160 & 71.02 & 39.95 & 23.96 & 34.83 & 22.02 & 44.49 & 33.99 & 26.35 & 27.75 & 49.28 \\
\hline 5401017 & 73 & 1956 & 2011 & 55 & $51-55$ & $\mathrm{~F}$ & 155 & 70.99 & 39.21 & 24.09 & 34.11 & 24.16 & 42.76 & 36.29 & 21.83 & 23.92 & 45.13 \\
\hline $184 / 52$ & 74 & 1954 & 2009 & 55 & $51-55$ & $\mathrm{~F}$ & 159 & 78.01 & 44.15 & 22.79 & 34.9 & 26.13 & 44.87 & 37.69 & 27.73 & 25.22 & 51.64 \\
\hline 5407110 & 75 & 1955 & 2011 & 56 & $56-60$ & $\mathrm{~F}$ & 150 & 75.18 & 37.47 & 23.98 & 33.16 & 23.72 & 43.53 & 35.25 & 25.51 & 23.77 & 50.42 \\
\hline $38 / 52$ & 76 & 1951 & 2009 & 58 & $56-60$ & $\mathrm{~F}$ & 150 & 69.25 & 36.15 & 21.15 & 32.22 & 22.04 & 41.13 & 34.46 & 24.24 & 23.26 & 44.22 \\
\hline $289 / 55$ & 77 & 1953 & 2012 & 59 & $56-60$ & $\mathrm{~F}$ & 150 & 71.36 & 40.01 & 26.25 & 32.73 & 22.89 & 43.08 & 35.22 & 27.64 & 25.8 & 46.47 \\
\hline 5305094 & 78 & 1951 & 2010 & 59 & $56-60$ & $\mathrm{~F}$ & 155 & 72.81 & 35.44 & 23.66 & 31.63 & 21.06 & 46.47 & 35.05 & 26.82 & 27.61 & 51.49 \\
\hline
\end{tabular}




\begin{tabular}{|c|c|c|c|c|c|c|c|c|c|c|c|c|c|c|c|c|c|}
\hline $\begin{array}{l}\text { Catalogue } \\
\text { No. }\end{array}$ & ID & $\begin{array}{l}\text { Date of } \\
\text { Birth }\end{array}$ & $\begin{array}{l}\text { Date of } \\
\text { Death }\end{array}$ & $\begin{array}{l}\text { Age } \\
\text { (years) }\end{array}$ & $\begin{array}{l}\text { Age Group } \\
\text { (years) }\end{array}$ & Sex & $\begin{array}{l}\text { Living } \\
\text { Height (cm) }\end{array}$ & $\begin{array}{l}\text { MAXL } \\
(\mathbf{m m})\end{array}$ & $\begin{array}{l}\text { MAXH } \\
(\mathbf{m m})\end{array}$ & $\begin{array}{l}\text { CFH } \\
(\mathbf{m m})\end{array}$ & $\begin{array}{l}\text { BH } \\
(\mathbf{m m})\end{array}$ & $\begin{array}{l}\text { MINB } \\
(\mathrm{mm})\end{array}$ & $\begin{array}{l}\text { LAL } \\
(\mathbf{m m})\end{array}$ & $\begin{array}{l}\text { MIDB } \\
(\mathrm{mm})\end{array}$ & $\begin{array}{l}\text { DAFB } \\
(\mathrm{mm})\end{array}$ & $\begin{array}{l}\text { DAFL } \\
(\mathrm{mm})\end{array}$ & $\begin{array}{l}\text { MTAL } \\
(\mathrm{mm})\end{array}$ \\
\hline $48 / 53$ & 79 & 1949 & 2010 & 61 & $61-65$ & $\mathrm{~F}$ & 150 & 73.96 & 35.48 & 21.53 & 31.94 & 21.1 & 45.83 & 34.83 & 24.86 & 23.7 & 47.66 \\
\hline $271 / 56$ & 80 & 1952 & 2013 & 61 & $61-65$ & $\mathrm{~F}$ & 155 & 71.91 & 37.66 & 22.89 & 32.23 & 20.85 & 44.07 & 36.49 & 24.06 & 25.03 & 52.63 \\
\hline $74 / 52$ & 81 & 1947 & 2009 & 62 & $61-65$ & F & 155 & 70.79 & 40.81 & 21.21 & 31.19 & 23.84 & 42.7 & 38.45 & 27.42 & 24.48 & 48.01 \\
\hline $77 / 50$ & 82 & 1945 & 2007 & 62 & $61-65$ & $\mathrm{~F}$ & 160 & 78.34 & 41.07 & 23.31 & 32.94 & 23.05 & 47.02 & 36.21 & 24.89 & 26.3 & 49.64 \\
\hline 5407126 & 83 & 1949 & 2011 & 62 & $61-65$ & F & 150 & 73.66 & 36.69 & 25.49 & 29.7 & 22.89 & 43.97 & 34.86 & 23.78 & 25.47 & 47.7 \\
\hline 5402023 & 84 & 1958 & 2011 & 53 & $51-55$ & $\mathrm{~F}$ & 165 & 82.38 & 43.07 & 28.47 & 41.21 & 30.7 & 46.71 & 44.14 & 30.96 & 31.66 & 59.27 \\
\hline $133 / 52$ & 85 & 1947 & 2009 & 62 & $61-65$ & F & 150 & 72.38 & 37.48 & 22.53 & 32.56 & 22.68 & 41.65 & 37.62 & 26.01 & 22.6 & 44.55 \\
\hline $108 / 50$ & 86 & 1944 & 2007 & 63 & $61-65$ & F & 145 & 68.49 & 38.08 & 22.12 & 29.03 & 21.72 & 39.7 & 36.67 & 23.25 & 23.56 & 47.25 \\
\hline $40 / 51$ & 87 & 1945 & 2008 & 63 & $61-65$ & F & 150 & 76.55 & 40.88 & 27.27 & 38.18 & 26.86 & 47.06 & 38.19 & 26.79 & 28.35 & 56.49 \\
\hline 199/51 & 88 & 1945 & 2008 & 63 & $61-65$ & $\mathrm{~F}$ & 150 & 74.11 & 35.01 & 23.29 & 32.23 & 26.09 & 43.29 & 37.65 & 27.19 & 25.95 & 46.69 \\
\hline $25 / 56$ & 89 & 1950 & 2013 & 63 & $61-65$ & $\mathrm{~F}$ & 150 & 68.12 & 35.54 & 22.61 & 34.09 & 21.45 & 43.1 & 34.65 & 25.61 & 26.41 & 46.34 \\
\hline 5409174 & 90 & 1947 & 2011 & 64 & $61-65$ & $\mathrm{~F}$ & 160 & 69.93 & 39.84 & 24.55 & 36.85 & 20.88 & 43.72 & 36.24 & 26.61 & 24.42 & 46.58 \\
\hline $49 / 50$ & 91 & 1942 & 2007 & 65 & $61-65$ & F & 170 & 84.05 & 45.72 & 24.01 & 35.3 & 25.54 & 47.12 & 38.01 & 26.26 & 29.15 & 52.65 \\
\hline $31 / 52$ & 92 & 1944 & 2009 & 65 & $61-65$ & F & 146 & 69.9 & 40.3 & 23.58 & 34.52 & 24 & 42.38 & 35.9 & 22.5 & 24.96 & 49.07 \\
\hline $53 / 52$ & 93 & 1944 & 2009 & 65 & $61-65$ & $\mathrm{~F}$ & 155 & 69.97 & 37.25 & 26.34 & 34.57 & 22.99 & 42.42 & 38.41 & 30.59 & 26.59 & 51.31 \\
\hline $84 / 50$ & 94 & 1941 & 2007 & 66 & $66-70$ & $\mathrm{~F}$ & 160 & 73.14 & 43.16 & 25.13 & 30.5 & 20.24 & 45.27 & 37.63 & 25.42 & 25.65 & 51.53 \\
\hline $151 / 52$ & 95 & 1943 & 2009 & 66 & $66-70$ & F & 140 & 67.01 & 38.04 & 23.24 & 31.08 & 24.07 & 41.28 & 34.12 & 25.18 & 24.1 & 45.28 \\
\hline 5212188 & 96 & 1943 & 2009 & 66 & $66-70$ & F & 160 & 75.17 & 39.06 & 25.92 & 33.78 & 24.22 & 44.91 & 36.16 & 28.22 & 27.25 & 50.45 \\
\hline 5312238 & 97 & 1943 & 2010 & 67 & $66-70$ & $\mathrm{~F}$ & 150 & 72.05 & 35.13 & 24.41 & 35.72 & 22.9 & 43.58 & 35.55 & 25.13 & 24.27 & 49.46 \\
\hline 5402033 & 98 & 1944 & 2011 & 67 & $66-70$ & F & 150 & 76.66 & 38.42 & 28.05 & 33.18 & 21.5 & 43.48 & 36.26 & 26.54 & 25.72 & 48.17 \\
\hline 5308140 & 99 & 1943 & 2010 & 67 & $66-70$ & $\mathrm{~F}$ & 145 & 70.4 & 37.42 & 23.47 & 32.45 & 23.57 & 42.63 & 38.63 & 25.96 & 27.52 & 48.17 \\
\hline $101 / 51$ & 100 & 1940 & 2008 & 68 & $66-70$ & $\mathrm{~F}$ & 140 & 75.48 & 37.35 & 27.37 & 34.51 & 21.41 & 41.78 & 37.68 & 23.26 & 24.72 & 47.67 \\
\hline $159 / 52$ & 101 & 1940 & 2009 & 69 & $66-70$ & $\mathrm{~F}$ & 150 & 72.89 & 40.41 & 26.52 & 32.05 & 25.15 & 43.49 & 36.83 & 28.08 & 25.65 & 49.92 \\
\hline 5404056 & 102 & 1942 & 2011 & 69 & $66-70$ & $\mathrm{~F}$ & 150 & 70.67 & 36.64 & 19.86 & 31.39 & 21.62 & 45.08 & 38.72 & 25.06 & 26.46 & 50.81 \\
\hline $61 / 50$ & 103 & 1938 & 2007 & 69 & $66-70$ & $\mathrm{~F}$ & 158 & 73.83 & 36.85 & 25.85 & 37.74 & 22.64 & 45.37 & 36.55 & 25.42 & 28.27 & 45.69 \\
\hline $164 / 52$ & 104 & 1939 & 2009 & 70 & $66-70$ & F & 150 & 71.77 & 38.87 & 24.06 & 30.17 & 19.99 & 42.12 & 34.33 & 26.86 & 25.21 & 49.24 \\
\hline
\end{tabular}




\begin{tabular}{|c|c|c|c|c|c|c|c|c|c|c|c|c|c|c|c|c|c|}
\hline $\begin{array}{l}\text { Catalogue } \\
\text { No. }\end{array}$ & ID & $\begin{array}{l}\text { Date of } \\
\text { Birth }\end{array}$ & $\begin{array}{l}\text { Date of } \\
\text { Death }\end{array}$ & $\begin{array}{l}\text { Age } \\
\text { (years) }\end{array}$ & $\begin{array}{l}\text { Age Group } \\
\text { (years) }\end{array}$ & Sex & $\begin{array}{l}\text { Living } \\
\text { Height (cm) }\end{array}$ & $\begin{array}{l}\text { MAXL } \\
(\mathrm{mm})\end{array}$ & $\begin{array}{l}\text { MAXH } \\
(\mathbf{m m})\end{array}$ & $\begin{array}{l}\text { CFH } \\
(\mathbf{m m})\end{array}$ & $\begin{array}{l}\text { BH } \\
(\mathbf{m m})\end{array}$ & $\begin{array}{l}\text { MINB } \\
(\mathbf{m m})\end{array}$ & $\begin{array}{l}\text { LAL } \\
(\mathbf{m m})\end{array}$ & $\begin{array}{l}\text { MIDB } \\
(\mathrm{mm})\end{array}$ & $\begin{array}{l}\text { DAFB } \\
(\mathrm{mm})\end{array}$ & $\begin{array}{l}\text { DAFL } \\
(\mathbf{m m})\end{array}$ & $\begin{array}{l}\text { MTAL } \\
(\mathbf{m m})\end{array}$ \\
\hline $44 / 52$ & 105 & 1938 & 2009 & 71 & $71-75$ & $\mathrm{~F}$ & 155 & 78.14 & 38.06 & 25.89 & 34.52 & 24 & 46.37 & 38.53 & 27.91 & 26.52 & 49.38 \\
\hline 5409168 & 106 & 1940 & 2011 & 71 & $71-75$ & $\mathrm{~F}$ & 140 & 67.61 & 39.8 & 24.3 & 31.63 & 25.15 & 41.02 & 36.45 & 25.58 & 24.76 & 49.32 \\
\hline $103 / 50$ & 107 & 1935 & 2007 & 72 & $71-75$ & $\mathrm{~F}$ & 145 & 72.66 & 41.13 & 24.59 & 33.54 & 24.52 & 46.56 & 36.28 & 25.84 & 28.1 & 48.96 \\
\hline $149 / 52$ & 108 & 1937 & 2009 & 72 & $71-75$ & F & 150 & 70.53 & 39.257 & 23.2 & 35.71 & 22.94 & 43.66 & 36.26 & 25.35 & 25.38 & 51.72 \\
\hline $57 / 53$ & 109 & 1938 & 2010 & 72 & $71-75$ & $\mathrm{~F}$ & 150 & 72.76 & 37.04 & 23.09 & 34.13 & 23.26 & 42.99 & 37.08 & 23.1 & 24.96 & 48.59 \\
\hline $32 / 56$ & 110 & 1941 & 2013 & 72 & $71-75$ & $\mathrm{~F}$ & 150 & 74.62 & 40.27 & 24.11 & 35.2 & 22.29 & 43.8 & 37.23 & 25.41 & 26.72 & 48.01 \\
\hline $42 / 52$ & 111 & 1936 & 2009 & 73 & $71-75$ & $\mathrm{~F}$ & 155 & 76.23 & 38.37 & 24.01 & 31.77 & 25.8 & 44.93 & 37.85 & 26.84 & 26.39 & 50.09 \\
\hline $76 / 52$ & 112 & 1936 & 2009 & 73 & $71-75$ & F & 150 & 78.67 & 41.38 & 24.44 & 38.16 & 25.9 & 44.24 & 38.52 & 25.66 & 24.99 & 49.4 \\
\hline $160 / 57$ & 113 & 1941 & 2014 & 73 & $71-75$ & $\mathrm{~F}$ & 145 & 69.91 & 33.61 & 22.97 & 30.66 & 21.45 & 40.39 & 35.33 & 24.75 & 24.01 & 47.96 \\
\hline $279 / 56$ & 114 & 1939 & 2013 & 74 & $71-75$ & F & 150 & 81.13 & 44.53 & 25.89 & 36.88 & 25.99 & 45.21 & 37.94 & 30.58 & 28.09 & 54.05 \\
\hline $99 / 49$ & 115 & 1931 & 2006 & 75 & $71-75$ & $\mathrm{~F}$ & 155 & 73.51 & 36.4 & 22.68 & 34.94 & 21.96 & 42.16 & 35.74 & 24.44 & 25.07 & 48.85 \\
\hline $102 / 49$ & 116 & 1931 & 2006 & 75 & $71-75$ & F & 160 & 72.44 & 36.31 & 23.13 & 33.65 & 19.67 & 41.06 & 35.79 & 24.16 & 25.75 & 48.78 \\
\hline $57 / 51$ & 117 & 1933 & 2008 & 75 & $71-75$ & $\mathrm{~F}$ & 155 & 76.2 & 43.14 & 25.17 & 35.47 & 25.19 & 44.34 & 41.1 & 27.76 & 27.74 & 52.1 \\
\hline $78 / 56$ & 118 & 1937 & 2013 & 76 & $76-80$ & $\mathrm{~F}$ & 152 & 74.88 & 39.84 & 24.21 & 33.56 & 24.64 & 44.26 & 35.53 & 25.76 & 25.85 & 51.8 \\
\hline $23 / 56$ & 119 & 1936 & 2013 & 77 & $76-80$ & $\mathrm{~F}$ & 150 & 74.09 & 38.57 & 21.58 & 33.53 & 22.44 & 43.89 & 35.18 & 24.22 & 25.38 & 46.17 \\
\hline $99 / 50$ & 120 & 1930 & 2007 & 77 & $76-80$ & $\mathrm{~F}$ & 153 & 67.4 & 37.86 & 25.2 & 33.48 & 25.89 & 43.82 & 37.31 & 26.1 & 26.49 & 47.33 \\
\hline $42 / 51$ & 121 & 1931 & 2008 & 77 & $76-80$ & $\mathrm{~F}$ & 150 & 74.46 & 36.6 & 25.32 & 33.04 & 23.53 & 42.9 & 38.2 & 26.72 & 25.24 & 50.12 \\
\hline $11 / 53$ & 122 & 1932 & 2010 & 78 & $76-80$ & $\mathrm{~F}$ & 155 & 73.67 & 37.18 & 22.37 & 32.97 & 24.9 & 43.95 & 36.71 & 25.82 & 26.42 & 45.92 \\
\hline $111 / 56$ & 123 & 1935 & 2013 & 78 & $76-80$ & $\mathrm{~F}$ & 156 & 76.21 & 46.41 & 23.16 & 33.77 & 23.17 & 48.55 & 39.4 & 24.74 & 28.81 & 55.83 \\
\hline 5311203 & 124 & 1930 & 2010 & 80 & $76-80$ & $\mathrm{~F}$ & 150 & 73.32 & 39.4 & 24.31 & 34.1 & 24.32 & 43.11 & 36.92 & 27.01 & 24.43 & 51.51 \\
\hline 5402035 & 125 & 1931 & 2011 & 80 & $76-80$ & $\mathrm{~F}$ & 153 & 77.91 & 44.34 & 26.24 & 37.13 & 21.96 & 47.85 & 39.64 & 26.11 & 25.82 & 51.8 \\
\hline $46 / 53$ & 126 & 1928 & 2010 & 82 & $81-85$ & $\mathrm{~F}$ & 155 & 76.65 & 36.52 & 23.49 & 34.37 & 22.9 & 41.49 & 37.09 & 25.75 & 25.15 & 46.21 \\
\hline $229 / 54$ & 127 & 1929 & 2011 & 82 & $81-85$ & $\mathrm{~F}$ & 140 & 75.56 & 36.58 & 23.42 & 34.72 & 22.36 & 41.23 & 37.03 & 25.19 & 25.49 & 45.3 \\
\hline 5401021 & 128 & 1929 & 2011 & 82 & $81-85$ & $\mathrm{~F}$ & 150 & 78.4 & 42.93 & 27.13 & 35.91 & 22.94 & 48.83 & 37.97 & 29.31 & 29.27 & 54.31 \\
\hline $149 / 55$ & 129 & 1930 & 2012 & 82 & $81-85$ & $\mathrm{~F}$ & 140 & 78.49 & 45.93 & 28.71 & 39.34 & 25 & 49.4 & 41.71 & 28.3 & 27.95 & 55.06 \\
\hline $114 / 49$ & 130 & 1923 & 2006 & 83 & $81-85$ & $\mathrm{~F}$ & 150 & 70.71 & 41.99 & 22.96 & 37.25 & 24.89 & 46.95 & 36.28 & 26.59 & 26.75 & 51.02 \\
\hline
\end{tabular}




\begin{tabular}{|c|c|c|c|c|c|c|c|c|c|c|c|c|c|c|c|c|c|}
\hline $\begin{array}{l}\text { Catalogue } \\
\text { No. }\end{array}$ & ID & $\begin{array}{l}\text { Date of } \\
\text { Birth }\end{array}$ & $\begin{array}{l}\text { Date of } \\
\text { Death }\end{array}$ & $\begin{array}{l}\text { Age } \\
\text { (years) }\end{array}$ & $\begin{array}{l}\text { Age Group } \\
\text { (years) }\end{array}$ & Sex & $\begin{array}{l}\text { Living } \\
\text { Height (cm) }\end{array}$ & $\begin{array}{l}\text { MAXL } \\
(\mathbf{m m})\end{array}$ & $\begin{array}{l}\text { MAXH } \\
(\mathbf{m m})\end{array}$ & $\begin{array}{l}\text { CFH } \\
(\mathbf{m m})\end{array}$ & $\begin{array}{l}\text { BH } \\
(\mathbf{m m})\end{array}$ & $\begin{array}{l}\text { MINB } \\
(\mathrm{mm})\end{array}$ & $\begin{array}{l}\text { LAL } \\
(\mathbf{m m})\end{array}$ & $\begin{array}{l}\text { MIDB } \\
(\mathrm{mm})\end{array}$ & $\begin{array}{l}\text { DAFB } \\
(\mathrm{mm})\end{array}$ & $\begin{array}{l}\text { DAFL } \\
(\mathrm{mm})\end{array}$ & $\begin{array}{l}\text { MTAL } \\
(\mathrm{mm})\end{array}$ \\
\hline $42 / 53$ & 131 & 1927 & 2010 & 83 & $81-85$ & F & 166 & 80.17 & 43.55 & 23.85 & 39.52 & 25.54 & 45.28 & 40.46 & 28.07 & 28.03 & 52.62 \\
\hline 5401008 & 132 & 1927 & 2011 & 84 & $81-85$ & $\mathrm{~F}$ & 150 & 75.39 & 30.14 & 25.59 & 34.43 & 24.83 & 42.27 & 36.53 & 25.88 & 26.24 & 47.67 \\
\hline $88 / 56$ & 133 & 1929 & 2013 & 84 & $81-85$ & F & 160 & 74.3 & 37.88 & 24.72 & 36.6 & 25.02 & 41.4 & 36.15 & 24.2 & 27.24 & 51 \\
\hline $385 / 56$ & 134 & 1928 & 2013 & 85 & $81-85$ & F & 150 & 68.12 & 27.24 & 21.77 & 31.51 & 19.37 & 40.57 & 31.93 & 23.41 & 22.89 & 47.19 \\
\hline $115 / 49$ & 135 & 1921 & 2006 & 85 & $81-85$ & F & 150 & 77.06 & 44.01 & 22.73 & 35.16 & 24.66 & 46.73 & 36.99 & 25.35 & 27.27 & 51.26 \\
\hline 5401020 & 136 & 1926 & 2011 & 85 & $81-85$ & $\mathrm{~F}$ & 153 & 76.46 & 41.68 & 22.52 & 37.72 & 21.55 & 48.19 & 39.93 & 27.37 & 27.74 & 53.78 \\
\hline $197 / 51$ & 137 & 1922 & 2008 & 86 & $86-90$ & F & 145 & 67.18 & 33.47 & 21.28 & 32.27 & 21.98 & 39.31 & 34.93 & 25.6 & 21.54 & 47.58 \\
\hline $55 / 52$ & 138 & 1923 & 2009 & 86 & $86-90$ & F & 150 & 69.83 & 37.39 & 24.3 & 32.33 & 22.11 & 42.62 & 35.61 & 25.75 & 25.56 & 48.17 \\
\hline 5406103 & 139 & 1924 & 2011 & 87 & $86-90$ & F & 140 & 67.41 & 36.41 & 22.71 & 34.16 & 20.65 & 42.66 & 34.66 & 24.09 & 25.72 & 49.64 \\
\hline 5405072 & 140 & 1923 & 2011 & 88 & $86-90$ & $\mathrm{~F}$ & 140 & 69.64 & 33.78 & 23.89 & 32.16 & 24.55 & 41.09 & 37.19 & 24.69 & 24.13 & 45.67 \\
\hline $3 / 57$ & 141 & 1926 & 2014 & 88 & $86-90$ & $\mathrm{~F}$ & 145 & 69.5 & 36.13 & 21.36 & 32.24 & 23.57 & 41.73 & 35.51 & 25.59 & 23.76 & 47.73 \\
\hline $113 / 49$ & 142 & 1915 & 2006 & 91 & $91-95$ & $\mathrm{~F}$ & 150 & 77.85 & 39.59 & 24.89 & 34.21 & 23.83 & 45.54 & 41.71 & 27.88 & 28.21 & 48.14 \\
\hline $191 / 54$ & 143 & 1975 & 2011 & 36 & $36-40$ & M & 165 & 83.59 & 46.16 & 27.95 & 38.78 & 25.89 & 51.23 & 41.29 & 29.53 & 29.84 & 59.16 \\
\hline $61 / 56$ & 144 & 1977 & 2013 & 36 & $36-40$ & M & 162 & 79.31 & 46.22 & 25.55 & 43.12 & 25.08 & 51.16 & 42.94 & 31.5 & 28.95 & 60.5 \\
\hline $181 / 52$ & 145 & 1972 & 2009 & 37 & $36-40$ & M & 165 & 77.03 & 42.52 & 25.88 & 38.06 & 22.4 & 46.49 & 38.35 & 27.96 & 26.74 & 54.73 \\
\hline $165 / 52$ & 146 & 1970 & 2009 & 39 & $36-40$ & M & 165 & 82.55 & 42.53 & 26.88 & 38.08 & 26.72 & 48.76 & 41.23 & 28.01 & 28.43 & 58.03 \\
\hline $55 / 53$ & 147 & 1969 & 2010 & 41 & $41-45$ & M & 170 & 77.22 & 40.93 & 26.75 & 36.72 & 25.81 & 48.1 & 41.46 & 30.78 & 29.77 & 58.3 \\
\hline $49 / 53$ & 148 & 1968 & 2010 & 42 & $41-45$ & M & 165 & 75.75 & 42.49 & 24.88 & 39.12 & 21.55 & 45.27 & 37.35 & 28.49 & 27.51 & 52.94 \\
\hline $328 / 56$ & 149 & 1970 & 2013 & 43 & $41-45$ & M & 160 & 75.36 & 39.8 & 23.69 & 37.24 & 25.63 & 45.81 & 38.99 & 26.94 & 27.69 & 54.02 \\
\hline $369 / 56$ & 150 & 1970 & 2013 & 43 & $41-45$ & M & 175 & 81.57 & 43.82 & 22.86 & 37.41 & 25.8 & 47.46 & 39.94 & 27.03 & 28.15 & 54.81 \\
\hline $35 / 50$ & 151 & 1963 & 2007 & 44 & $41-45$ & M & 176 & 77.59 & 44.88 & 26.09 & 40.18 & 27.01 & 48.06 & 39.15 & 28.59 & 29.09 & 60.47 \\
\hline $53 / 50$ & 152 & 1963 & 2007 & 44 & $41-45$ & M & 168 & 86.68 & 49.58 & 29.34 & 39.64 & 27.16 & 52.11 & 40.57 & 30.95 & 31.25 & 59.2 \\
\hline $145 / 50$ & 153 & 1963 & 2007 & 44 & $41-45$ & M & 160 & 79.39 & 38.7 & 22.16 & 40.91 & 26.1 & 48.94 & 41.6 & 26.65 & 28.53 & 51.95 \\
\hline 5408137 & 154 & 1964 & 2011 & 47 & $46-50$ & M & 150 & 75.52 & 41.49 & 26.96 & 39.9 & 23.43 & 45.87 & 38.66 & 30.96 & 28.51 & 55.43 \\
\hline $67 / 55$ & 155 & 1964 & 2012 & 48 & $46-50$ & M & 170 & 87.09 & 47.15 & 26.41 & 39.73 & 23.44 & 52.42 & 41.4 & 32.49 & 30.9 & 60.14 \\
\hline $256 / 55$ & 156 & 1956 & 2012 & 56 & $56-60$ & M & 172 & 83.03 & 43.3 & 25.59 & 41.66 & 24.65 & 51.17 & 40.27 & 30.27 & 29.47 & 59.28 \\
\hline
\end{tabular}




\begin{tabular}{|c|c|c|c|c|c|c|c|c|c|c|c|c|c|c|c|c|c|}
\hline $\begin{array}{l}\text { Catalogue } \\
\text { No. }\end{array}$ & ID & $\begin{array}{l}\text { Date of } \\
\text { Birth }\end{array}$ & $\begin{array}{l}\text { Date of } \\
\text { Death }\end{array}$ & $\begin{array}{l}\text { Age } \\
\text { (years) }\end{array}$ & $\begin{array}{l}\text { Age Group } \\
\text { (years) }\end{array}$ & Sex & $\begin{array}{l}\text { Living } \\
\text { Height (cm) }\end{array}$ & $\begin{array}{l}\text { MAXL } \\
(\mathrm{mm})\end{array}$ & $\begin{array}{l}\text { MAXH } \\
(\mathbf{m m})\end{array}$ & $\begin{array}{l}\text { CFH } \\
(\mathbf{m m})\end{array}$ & $\begin{array}{l}\text { BH } \\
(\mathrm{mm})\end{array}$ & $\begin{array}{l}\text { MINB } \\
(\mathbf{m m})\end{array}$ & $\begin{array}{l}\text { LAL } \\
(\mathbf{m m})\end{array}$ & $\begin{array}{l}\text { MIDB } \\
(\mathrm{mm})\end{array}$ & $\begin{array}{l}\text { DAFB } \\
(\mathbf{m m})\end{array}$ & $\begin{array}{l}\text { DAFL } \\
(\mathbf{m m})\end{array}$ & $\begin{array}{l}\text { MTAL } \\
(\mathrm{mm})\end{array}$ \\
\hline $15 / 56$ & 157 & 1956 & 2013 & 57 & $56-60$ & M & 165 & 73.88 & 39.11 & 25.75 & 34.7 & 23.73 & 45.14 & 38.5 & 29.95 & 27.8 & 57.78 \\
\hline $150 / 57$ & 158 & 1956 & 2014 & 58 & $56-60$ & M & 176 & 88.37 & 44.89 & 28.36 & 43.01 & 26.46 & 50.02 & 40.47 & 30.73 & 30.6 & 58.52 \\
\hline $75 / 50$ & 159 & 1949 & 2007 & 58 & $56-60$ & M & 170 & 82.66 & 48.28 & 26.1 & 41.04 & 23.87 & 54.74 & 41.13 & 28.76 & 26.33 & 63.19 \\
\hline 5404051 & 160 & 1952 & 2011 & 59 & $56-60$ & M & 165 & 79.32 & 45.66 & 26.58 & 41.46 & 23.53 & 46.5 & 38.45 & 30.2 & 28.06 & 53.2 \\
\hline 5402024 & 161 & 1951 & 2011 & 60 & $56-60$ & M & 165 & 79.04 & 44.61 & 26.85 & 40.28 & 26.12 & 45.44 & 40.74 & 27.74 & 28.78 & 58.57 \\
\hline $54 / 50$ & 162 & 1946 & 2007 & 61 & $61-65$ & M & 180 & 84.07 & 47.95 & 25.1 & 41.9 & 27.64 & 49.41 & 44.18 & 30.01 & 27.74 & 60.89 \\
\hline $104 / 50$ & 163 & 1946 & 2007 & 61 & $61-65$ & M & 164 & 76.76 & 40.48 & 22.91 & 35.35 & 25.69 & 44.6 & 40.05 & 25.33 & 22.13 & 52.74 \\
\hline 5409159 & 164 & 1950 & 2011 & 61 & $61-65$ & M & 165 & 85.31 & 43.5 & 25.76 & 41.9 & 28.02 & 49.58 & 43.53 & 29.98 & 31.06 & 58.48 \\
\hline $185 / 57$ & 165 & 1953 & 2014 & 61 & $61-65$ & M & 170 & 78.63 & 41.29 & 25.55 & 37.58 & 28.95 & 49.12 & 41.51 & 29.95 & 32.48 & 53.73 \\
\hline $27 / 52$ & 166 & 1947 & 2009 & 62 & $61-65$ & M & 175 & 81.64 & 43.72 & 26.72 & 42.44 & 26.19 & 50.89 & 46.21 & 30.39 & 30.58 & 60.38 \\
\hline 5405084 & 167 & 1949 & 2011 & 62 & $61-65$ & M & 166 & 81.75 & 42.7 & 28.24 & 37.52 & 25.45 & 48.69 & 42.78 & 28.51 & 29.32 & 55.8 \\
\hline $266 / 55$ & 168 & 1950 & 2012 & 62 & $61-65$ & M & 180 & 80.81 & 45.23 & 25.73 & 37.87 & 24.95 & 50.85 & 41.96 & 27.74 & 29.81 & 61.47 \\
\hline $41 / 50$ & 169 & 1944 & 2007 & 63 & $61-65$ & M & 170 & 80.86 & 38.05 & 27.78 & 36.98 & 26.11 & 47.58 & 41.95 & 25.08 & 27.56 & 49.54 \\
\hline $65 / 51$ & 170 & 1945 & 2008 & 63 & $61-65$ & M & 170 & 86.29 & 43.25 & 25.92 & 36 & 23.39 & 50.32 & 40.64 & 28.41 & 27.57 & 59.94 \\
\hline $71 / 52$ & 171 & 1945 & 2009 & 64 & $61-65$ & M & 170 & 84.42 & 45.9 & 29.53 & 38.88 & 27.52 & 50.18 & 40.16 & 29.45 & 30.37 & 59.8 \\
\hline 5406097 & 172 & 1947 & 2011 & 64 & $61-65$ & M & 160 & 76.38 & 34.45 & 22 & 41.17 & 23.95 & 46.67 & 41.7 & 31.19 & 30.65 & 55.1 \\
\hline $101 / 52$ & 173 & 1945 & 2009 & 64 & $61-65$ & M & 164 & 82.07 & 41.81 & 28.88 & 39.02 & 25.78 & 49.03 & 41.27 & 30.37 & 30.58 & 58.03 \\
\hline $4 / 50$ & 174 & 1942 & 2007 & 65 & $61-65$ & M & 166 & 80.38 & 45.82 & 26.27 & 38.98 & 25.5 & 48.76 & 41.65 & 31.73 & 30.64 & 56.63 \\
\hline $185 / 55$ & 175 & 1947 & 2012 & 65 & $61-65$ & M & 153 & 78.97 & 41.77 & 27 & 34.88 & 26.17 & 45.11 & 40.15 & 28.97 & 26.55 & 51.8 \\
\hline $60 / 51$ & 176 & 1943 & 2008 & 65 & $61-65$ & $\mathrm{M}$ & 180 & 90.29 & 44.74 & 29.59 & 41.87 & 26.77 & 52.79 & 39.63 & 29.05 & 28.66 & 58.63 \\
\hline $66 / 56$ & 177 & 1948 & 2013 & 65 & $61-65$ & $\mathrm{M}$ & 170 & 80.65 & 46.32 & 27.64 & 37.85 & 23.53 & 48.47 & 40.53 & 29.8 & 29.75 & 54.06 \\
\hline $29 / 50$ & 178 & 1940 & 2007 & 67 & $66-70$ & $\mathrm{M}$ & 170 & 81.81 & 42.72 & 28.18 & 36.97 & 24.7 & 48.98 & 38.74 & 31.09 & 29.88 & 56.15 \\
\hline $83 / 51$ & 179 & 1941 & 2008 & 67 & $66-70$ & $\mathrm{M}$ & 180 & 87.7 & 48.78 & 29.64 & 42.18 & 27.36 & 50.06 & 41.32 & 34.44 & 30.89 & 58.62 \\
\hline $47 / 53$ & 180 & 1943 & 2010 & 67 & $66-70$ & $\mathrm{M}$ & 165 & 76.92 & 43.67 & 24.96 & 39.16 & 24.98 & 49.38 & 39.92 & 26.43 & 28.93 & 57.46 \\
\hline $78 / 53$ & 181 & 1942 & 2010 & 68 & $66-70$ & M & 161 & 77.31 & 43.38 & 25.71 & 38.28 & 27.97 & 48.41 & 40.93 & 30.62 & 29.4 & 54.13 \\
\hline $31 / 50$ & 182 & 1938 & 2007 & 69 & $66-70$ & $\mathrm{M}$ & 160 & 77.42 & 42.1 & 26.58 & 39.31 & 25.01 & 49.12 & 40.33 & 28.44 & 29.43 & 54.77 \\
\hline
\end{tabular}




\begin{tabular}{|c|c|c|c|c|c|c|c|c|c|c|c|c|c|c|c|c|c|}
\hline $\begin{array}{l}\text { Catalogue } \\
\text { No. }\end{array}$ & ID & $\begin{array}{l}\text { Date of } \\
\text { Birth }\end{array}$ & $\begin{array}{l}\text { Date of } \\
\text { Death }\end{array}$ & $\begin{array}{l}\text { Age } \\
\text { (years) }\end{array}$ & $\begin{array}{l}\text { Age Group } \\
\text { (years) }\end{array}$ & Sex & $\begin{array}{l}\text { Living } \\
\text { Height (cm) }\end{array}$ & $\begin{array}{l}\text { MAXL } \\
(\mathbf{m m})\end{array}$ & $\begin{array}{l}\text { MAXH } \\
(\mathbf{m m})\end{array}$ & $\begin{array}{l}\text { CFH } \\
(\mathbf{m m})\end{array}$ & $\begin{array}{l}\text { BH } \\
(\mathbf{m m})\end{array}$ & $\begin{array}{l}\text { MINB } \\
(\mathrm{mm})\end{array}$ & $\begin{array}{l}\text { LAL } \\
(\mathbf{m m})\end{array}$ & $\begin{array}{l}\text { MIDB } \\
(\mathrm{mm})\end{array}$ & $\begin{array}{l}\text { DAFB } \\
(\mathrm{mm})\end{array}$ & $\begin{array}{l}\text { DAFL } \\
(\mathrm{mm})\end{array}$ & $\begin{array}{l}\text { MTAL } \\
(\mathrm{mm})\end{array}$ \\
\hline $81 / 51$ & 183 & 1939 & 2008 & 69 & $66-70$ & M & 180 & 84.34 & 47.16 & 28.05 & 41.74 & 25.68 & 48.83 & 44.61 & 29.71 & 28.81 & 59.75 \\
\hline $18 / 53$ & 184 & 1941 & 2010 & 69 & $66-70$ & M & 180 & 91.86 & 51 & 31.57 & 42.39 & 30.26 & 54.26 & 42.38 & 32.06 & 33.07 & 67.08 \\
\hline $41 / 54$ & 185 & 1942 & 2011 & 69 & $66-70$ & M & 157 & 79.6 & 45.9 & 29.34 & 39.86 & 24.17 & 48.26 & 41.4 & 29.53 & 28.37 & 54.94 \\
\hline $110 / 49$ & 186 & 1935 & 2006 & 71 & $71-75$ & M & 165 & 83.88 & 45.18 & 25.5 & 41.35 & 25.71 & 48.88 & 42.66 & 27.59 & 26.11 & 59.61 \\
\hline $118 / 49$ & 187 & 1935 & 2006 & 71 & $71-75$ & M & 165 & 71.45 & 31.32 & 23.62 & 34.69 & 24.29 & 42.32 & 35.7 & 26.53 & 27.24 & 48.2 \\
\hline $13 / 50$ & 188 & 1935 & 2007 & 72 & $71-75$ & M & 170 & 82.14 & 48.67 & 24.78 & 39.03 & 25.62 & 49.08 & 42 & 32.89 & 29.6 & 55.31 \\
\hline $62 / 50$ & 189 & 1935 & 2007 & 72 & $71-75$ & M & 170 & 85.89 & 41.43 & 26.6 & 39.15 & 25.8 & 53.62 & 42.32 & 30.55 & 31.55 & 58.08 \\
\hline 5307124 & 190 & 1937 & 2010 & 73 & $71-75$ & M & 170 & 78.18 & 47.61 & 24.76 & 35.85 & 23.92 & 51.03 & 40.95 & 26.42 & 28.5 & 53.92 \\
\hline 5405079 & 191 & 1938 & 2011 & 73 & $71-75$ & M & 160 & 79.75 & 43.89 & 26.28 & 39.78 & 26.32 & 49.37 & 41.19 & 28.57 & 29.13 & 55.22 \\
\hline $54 / 56$ & 192 & 1940 & 2013 & 73 & $71-75$ & M & 165 & 87.3 & 47.69 & 28.61 & 42.88 & 28.07 & 53.63 & 42.32 & 31.84 & 33.9 & 58.22 \\
\hline $43 / 53$ & 193 & 1936 & 2010 & 74 & $71-75$ & M & 170 & 82.31 & 45.99 & 27.78 & 45.48 & 23.84 & 49.93 & 39.96 & 27.5 & 28.46 & 58.61 \\
\hline $8 / 53$ & 194 & 1935 & 2010 & 75 & $71-75$ & M & 150 & 76.18 & 41.15 & 22.17 & 36.06 & 24.8 & 45.2 & 39.23 & 25.43 & 26.42 & 51.09 \\
\hline $18 / 52$ & 195 & 1934 & 2009 & 75 & $71-75$ & M & 160 & 81.74 & 42.12 & 28.97 & 39.57 & 25.01 & 48.37 & 43.83 & 31.73 & 32.57 & 58.82 \\
\hline $192 / 52$ & 196 & 1934 & 2009 & 75 & $71-75$ & M & 166 & 81.24 & 44.14 & 27.46 & 37.58 & 22.1 & 49.89 & 41.6 & 24.56 & 28.61 & 59.59 \\
\hline 5304080 & 197 & 1934 & 2010 & 76 & $76-80$ & M & 153 & 74.19 & 45.62 & 27.22 & 35.56 & 24.08 & 47.76 & 39.11 & 25.77 & 29.15 & 55.19 \\
\hline $120 / 56$ & 198 & 1937 & 2013 & 76 & $76-80$ & M & 167 & 86.68 & 51.94 & 32.29 & 38.8 & 27.07 & 51.67 & 40.31 & 28.53 & 28.69 & 57.01 \\
\hline $63 / 50$ & 199 & 1929 & 2007 & 78 & $76-80$ & M & 165 & 78.32 & 43.42 & 28.35 & 34.33 & 27.45 & 53.58 & 46.57 & 32.31 & 31.95 & 57.01 \\
\hline $61 / 53$ & 200 & 1932 & 2010 & 78 & $76-80$ & M & 156 & 72.54 & 37.81 & 23.26 & 35.83 & 23.37 & 44.34 & 35.1 & 27.2 & 26.17 & 50.27 \\
\hline $134 / 50$ & 201 & 1927 & 2007 & 80 & $76-80$ & M & 160 & 86.42 & 47.81 & 28.89 & 41.21 & 24.14 & 52.78 & 40.56 & 29.62 & 30.64 & 56.67 \\
\hline 5404066 & 202 & 1930 & 2011 & 81 & $81-85$ & M & 155 & 72.84 & 43.01 & 26.37 & 32.88 & 25.26 & 45.87 & 37.59 & 26.8 & 27.29 & 50.57 \\
\hline 5401003 & 203 & 1930 & 2011 & 81 & $81-85$ & M & 165 & 80.22 & 43.18 & 25.69 & 38.81 & 24.77 & 48.6 & 37.34 & 27.73 & 28.02 & 56.85 \\
\hline $288 / 55$ & 204 & 1931 & 2012 & 81 & $81-85$ & M & 160 & 76.59 & 43.86 & 26.78 & 40.74 & 24.3 & 47.02 & 40.72 & 28.05 & 29.94 & 52.91 \\
\hline $2 / 53$ & 205 & 1928 & 2010 & 82 & $81-85$ & M & 160 & 78.7 & 43.98 & 25.1 & 38.08 & 25 & 45.82 & 39.11 & 27.5 & 31.21 & 54.12 \\
\hline $48 / 56$ & 206 & 1931 & 2013 & 82 & $81-85$ & M & 155 & 78.33 & 38.73 & 26.56 & 35.34 & 22.92 & 46.76 & 39.16 & 27.39 & 28.05 & 53.57 \\
\hline $76 / 50$ & 207 & 1924 & 2007 & 83 & $81-85$ & M & 165 & 75.97 & 41.58 & 27.28 & 36.03 & 26.57 & 46.67 & 41.15 & 31.19 & 29.79 & 54.78 \\
\hline $5 / 54$ & 208 & 1927 & 2011 & 84 & $81-85$ & M & 167 & 79.74 & 45.2 & 27.71 & 35.91 & 23.86 & 44.82 & 38.39 & 28.06 & 25.46 & 54.17 \\
\hline
\end{tabular}




\begin{tabular}{|c|c|c|c|c|c|c|c|c|c|c|c|c|c|c|c|c|c|}
\hline $\begin{array}{l}\text { Catalogue } \\
\text { No. }\end{array}$ & ID & $\begin{array}{l}\text { Date of } \\
\text { Birth }\end{array}$ & $\begin{array}{l}\text { Date of } \\
\text { Death }\end{array}$ & $\begin{array}{l}\text { Age } \\
\text { (years) }\end{array}$ & $\begin{array}{l}\text { Age Group } \\
\text { (years) }\end{array}$ & Sex & $\begin{array}{l}\text { Living } \\
\text { Height (cm) }\end{array}$ & $\begin{array}{l}\text { MAXL } \\
(\mathbf{m m})\end{array}$ & $\begin{array}{l}\text { MAXH } \\
(\mathbf{m m})\end{array}$ & $\begin{array}{l}\text { CFH } \\
(\mathbf{m m})\end{array}$ & $\begin{array}{l}\text { BH } \\
(\mathbf{m m})\end{array}$ & $\begin{array}{l}\text { MINB } \\
(\mathrm{mm})\end{array}$ & $\begin{array}{l}\text { LAL } \\
(\mathbf{m m})\end{array}$ & $\begin{array}{l}\text { MIDB } \\
(\mathrm{mm})\end{array}$ & $\begin{array}{l}\text { DAFB } \\
(\mathrm{mm})\end{array}$ & $\begin{array}{l}\text { DAFL } \\
(\mathrm{mm})\end{array}$ & $\begin{array}{l}\text { MTAL } \\
(\mathrm{mm})\end{array}$ \\
\hline $32 / 50$ & 209 & 1922 & 2007 & 85 & $81-85$ & $\mathrm{M}$ & 168 & 75.65 & 42.52 & 26.11 & 36.12 & 24.5 & 48.75 & 40.43 & 28.44 & 29.78 & 50.37 \\
\hline $24 / 53$ & 210 & 1925 & 2010 & 85 & $81-85$ & $\mathrm{M}$ & 167 & 80.53 & 46.33 & 24.53 & 34.81 & 26.81 & 47.86 & 44.21 & 31.58 & 29.05 & 60.93 \\
\hline $15 / 50$ & 211 & 1922 & 2007 & 85 & $81-85$ & M & 155 & 69.11 & 42.95 & 25.33 & 38.78 & 24.98 & 42.61 & 40.69 & 26.89 & 26.67 & 50.34 \\
\hline 5409160 & 212 & 1926 & 2011 & 85 & $81-85$ & $\mathrm{M}$ & 170 & 87.79 & 37.29 & 29.13 & 39.54 & 25.27 & 47.18 & 38.97 & 26.82 & 27.42 & 57.33 \\
\hline $171 / 55$ & 213 & 1927 & 2012 & 85 & $81-85$ & M & 180 & 81.8 & 42.17 & 27.6 & 38.82 & 27.47 & 49.26 & 37.05 & 23.4 & 31.06 & 53.46 \\
\hline $347 / 56$ & 214 & 1928 & 2013 & 85 & $81-85$ & $\mathrm{M}$ & 155 & 77.22 & 41.07 & 29.62 & 35.54 & 24.77 & 45.85 & 40.57 & 26.12 & 27.25 & 53.79 \\
\hline $9 / 52$ & 215 & 1923 & 2009 & 86 & $86-90$ & M & 170 & 78.85 & 41.44 & 26.27 & 37.89 & 29.99 & 47.56 & 45.28 & 29.88 & 27.67 & 57.71 \\
\hline $62 / 53$ & 216 & 1924 & 2010 & 86 & $86-90$ & $\mathrm{M}$ & 155 & 76.16 & 40.12 & 26.67 & 37.5 & 23.43 & 45.23 & 40.33 & 24.47 & 29.49 & 48.98 \\
\hline $55 / 56$ & 217 & 1927 & 2013 & 86 & $86-90$ & M & 160 & 81.5 & 44.74 & 27.17 & 38.4 & 27.18 & 50.28 & 42.12 & 30.66 & 29.22 & 55.29 \\
\hline $143 / 50$ & 218 & 1920 & 2007 & 87 & $86-90$ & $\mathrm{M}$ & 160 & 80.23 & 46.47 & 26.97 & 41.06 & 24.26 & 48.24 & 40.02 & 30.02 & 30.94 & 57.46 \\
\hline $178 / 51$ & 219 & 1921 & 2008 & 87 & $86-90$ & M & 175 & 85.67 & 46.11 & 31.84 & 39.59 & 28.17 & 48.99 & 42.88 & 29.92 & 30.34 & 58.15 \\
\hline 5406096 & 220 & 1923 & 2011 & 88 & $86-90$ & M & 165 & 84.84 & 47.18 & 27.06 & 39.34 & 24.63 & 53.24 & 40.71 & 28.53 & 29.06 & 59.95 \\
\hline 5409180 & 221 & 1923 & 2011 & 88 & $86-90$ & M & 160 & 82.06 & 43.55 & 25.63 & 39.29 & 25.9 & 49.49 & 39.23 & 27.04 & 29.87 & 55.38 \\
\hline $25 / 51$ & 222 & 1917 & 2008 & 91 & $91-95$ & M & 170 & 83.51 & 43.66 & 25.68 & 43.03 & 27.28 & 52.53 & 44.67 & 31.19 & 31 & 59.24 \\
\hline $21 / 53$ & 223 & 1919 & 2010 & 91 & $91-95$ & M & 178 & 82.41 & 48.57 & 23.02 & 43.64 & 25.91 & 51.82 & 42.02 & 30.31 & 28.72 & 57.7 \\
\hline $5 / 50$ & 224 & 1913 & 2007 & 94 & $91-95$ & M & 160 & 78.38 & 41.59 & 25.04 & 35.82 & 26.54 & 46.22 & 43.92 & 30.66 & 28.91 & 56.54 \\
\hline $230 / 54$ & 225 & 1917 & 2011 & 94 & $91-95$ & M & 175 & 85.58 & 51.98 & 28.69 & 43.24 & 27.32 & 48.84 & 40.08 & 29.4 & 32.25 & 60.36 \\
\hline 5312243 & 226 & 1929 & 2010 & 81 & $81-85$ & F & 148 & 68.58 & 41.12 & 22.69 & 36.8 & 22.99 & 41.96 & 35.72 & 25.3 & 25.22 & 48.74 \\
\hline $41 / 56$ & 227 & 1940 & 2013 & 73 & $71-75$ & M & 170 & 72.54 & 38.84 & 26.33 & 36.05 & 27.77 & 43.83 & 39.65 & 27.95 & 26.1 & 51.56 \\
\hline 5407117 & 228 & 1951 & 2011 & 60 & $56-60$ & F & 150 & 67.87 & 39.74 & 23.9 & 34.24 & 22.39 & 42.98 & 37.35 & 24.8 & 25.28 & 48.39 \\
\hline $89 / 50$ & 229 & 1936 & 2007 & 71 & $71-75$ & $\mathrm{~F}$ & 150 & 79.74 & 39.9 & 28.11 & 33.92 & 25.6 & 49.4 & 41.93 & 27.16 & 28.79 & 51.52 \\
\hline 5406095 & 230 & 1927 & 2011 & 84 & $81-85$ & M & 160 & 82.08 & 44.61 & 27.44 & 35.64 & 22.58 & 46.93 & 41.39 & 28.2 & 29.32 & 55.93 \\
\hline $39 / 50$ & 231 & 1951 & 2007 & 56 & $56-60$ & M & 170 & 84.63 & 47.75 & 27.52 & 37.24 & 28.91 & 49.83 & 43.33 & 32.43 & 30.62 & 62.1 \\
\hline $186 / 52$ & 232 & 1926 & 2009 & 83 & $81-85$ & $\mathrm{~F}$ & 153 & 76.84 & 38.97 & 24.3 & 33.86 & 23.42 & 45.62 & 37.35 & 28.24 & 27.6 & 50.54 \\
\hline $121 / 50$ & 233 & 1938 & 2007 & 69 & $65-70$ & M & 170 & 82.66 & 46.7 & 28.27 & 40.19 & 27.1 & 47.78 & 40.16 & 28.36 & 28.82 & 54.78 \\
\hline
\end{tabular}


APPENDIX B:

EXAMPLES OF CALCANEAL AND TALAR PATHOLOGIES 


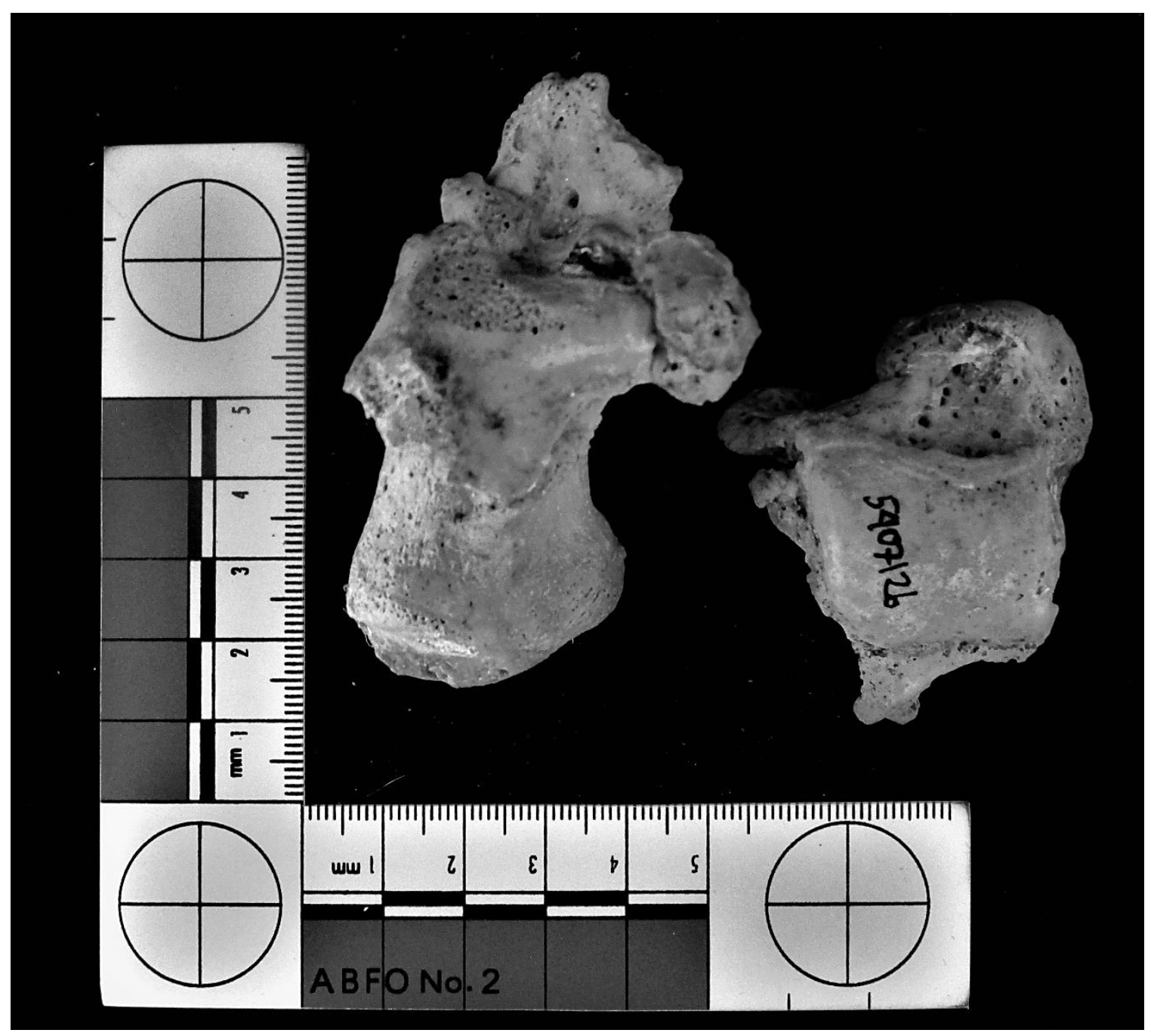

Appendix B1: Example \#1 of pathological left calcaneus and left talus, excluded from analyses.

(Catalogue number: 5407126) (Photo by: Shelby Scott) 


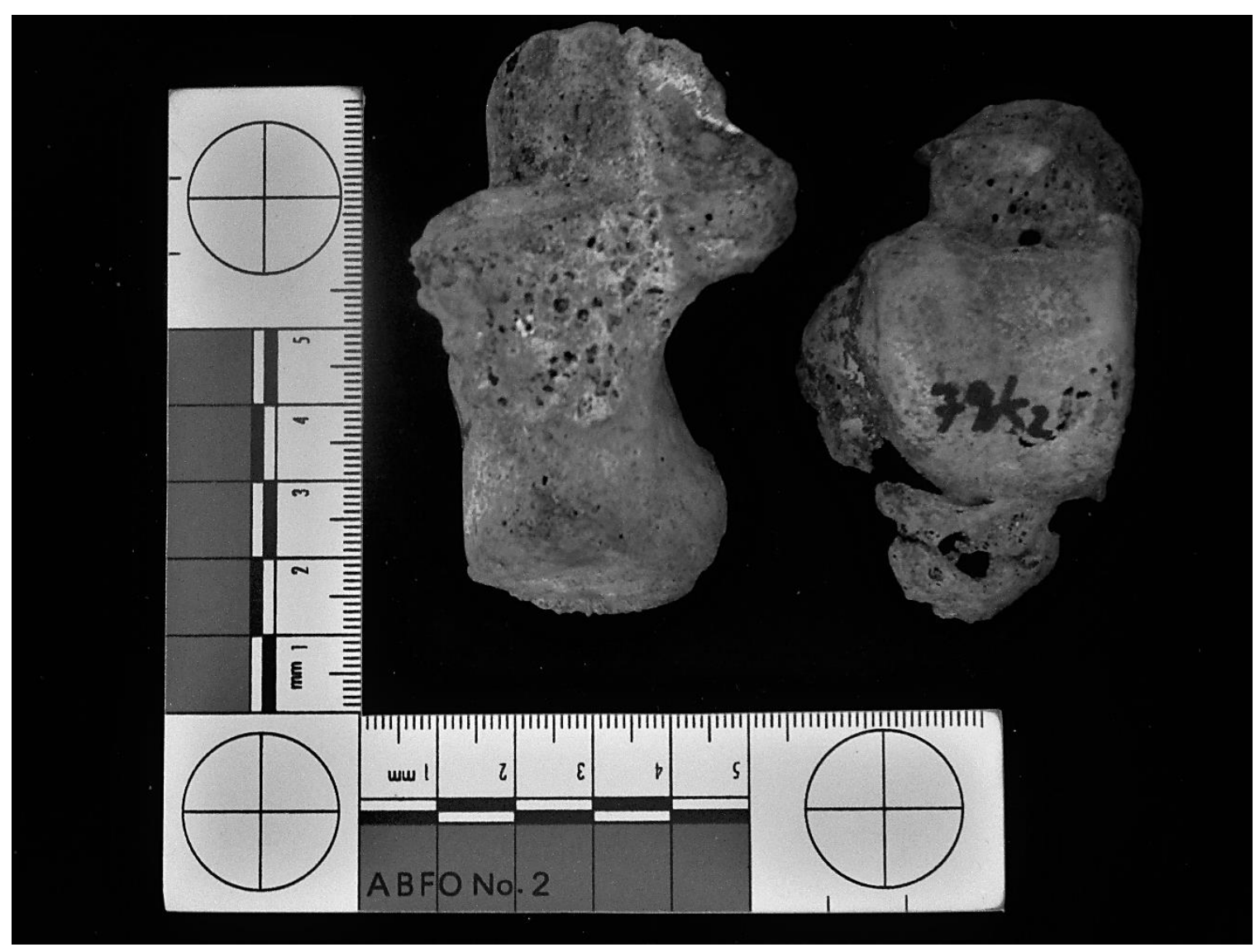

Appendix B2: Example \#2 of pathological left calcaneus and left talus, excluded from analyses.

(Catalogue number: 79-52) (Photo by: Shelby Scott) 


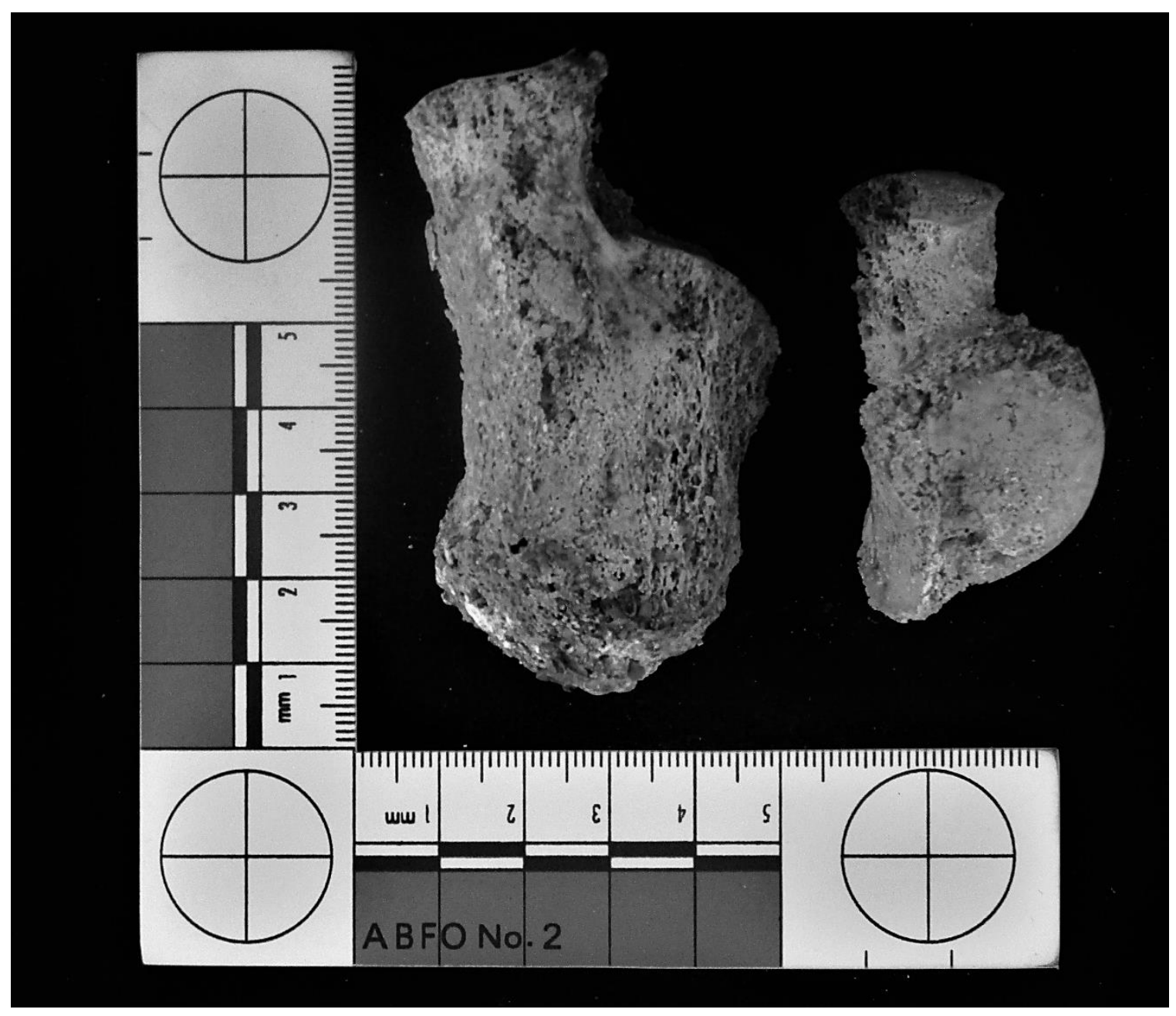

Appendix B3: Example of degraded left calcaneus and left talus, excluded from analyses.

(Catalogue number: 271-56) (Photo by: Shelby Scott) 


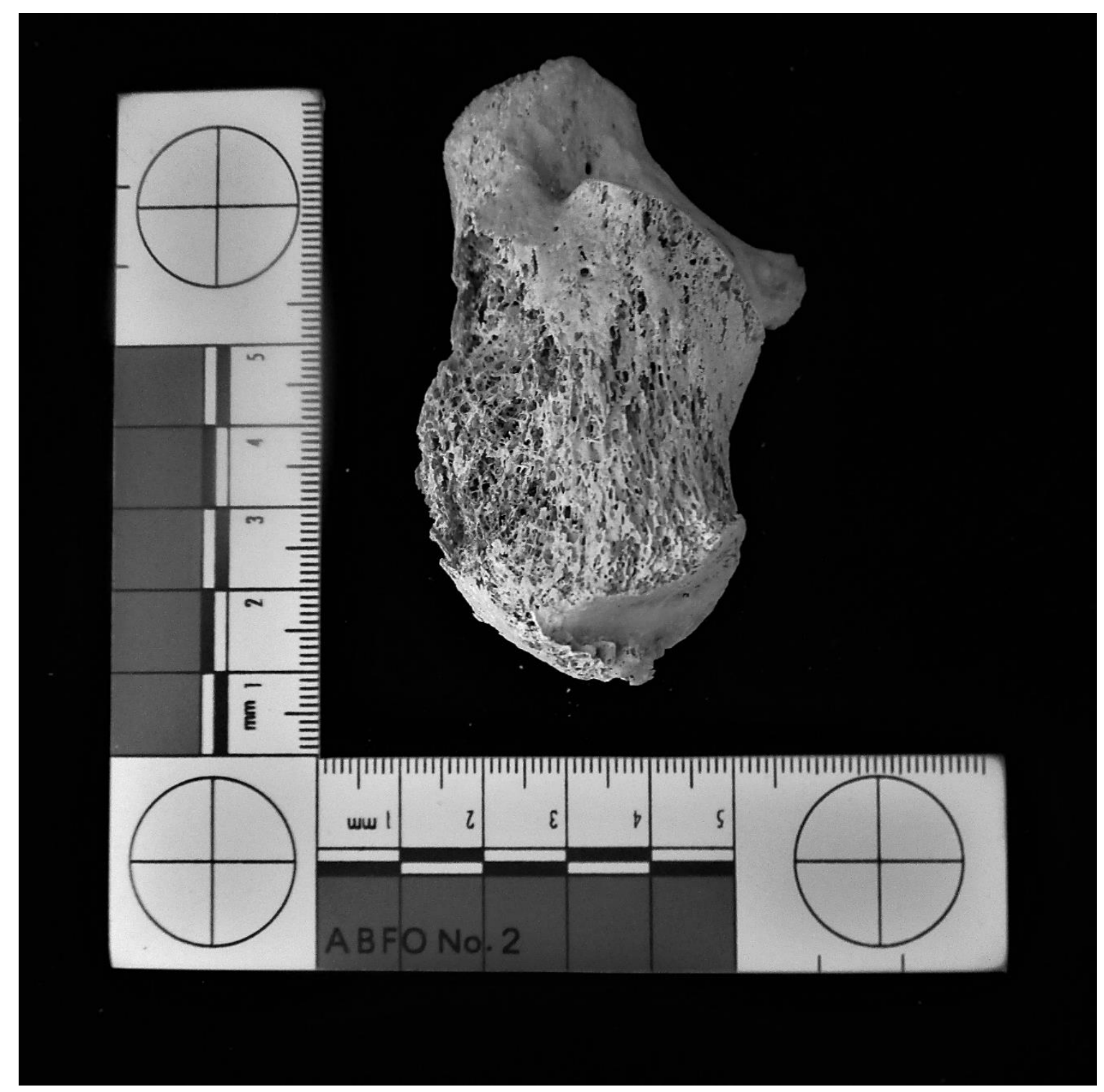

Appendix B4: Example of degraded left calcaneus, excluded from analyses. (Catalogue number: 5301017) (Photo by: Shelby Scott) 
APPENDIX C:

NORMALITY TESTS 


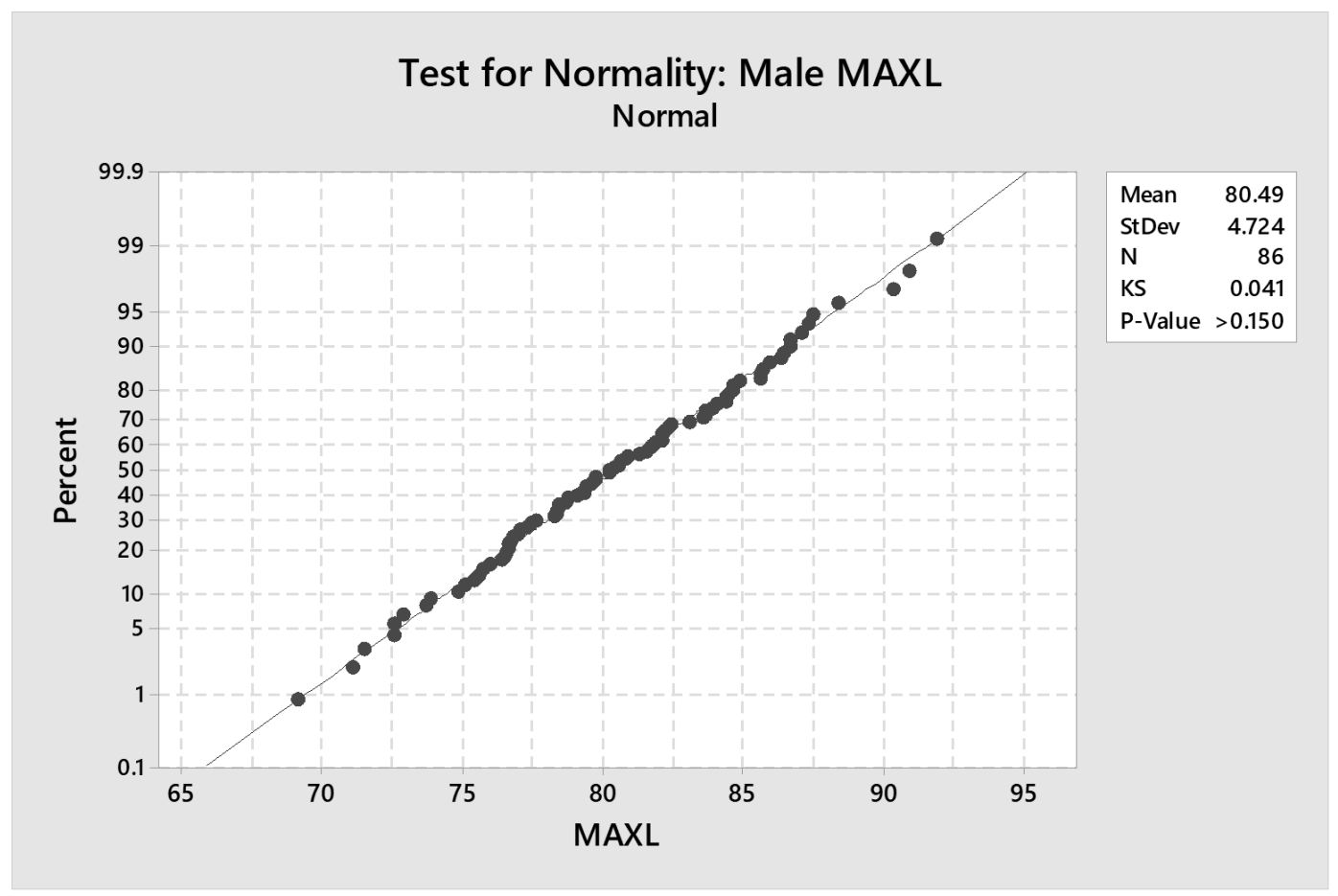

Appendix C1: Normality probability plot for male MAXL (Maximum Length) calcaneal measurements.

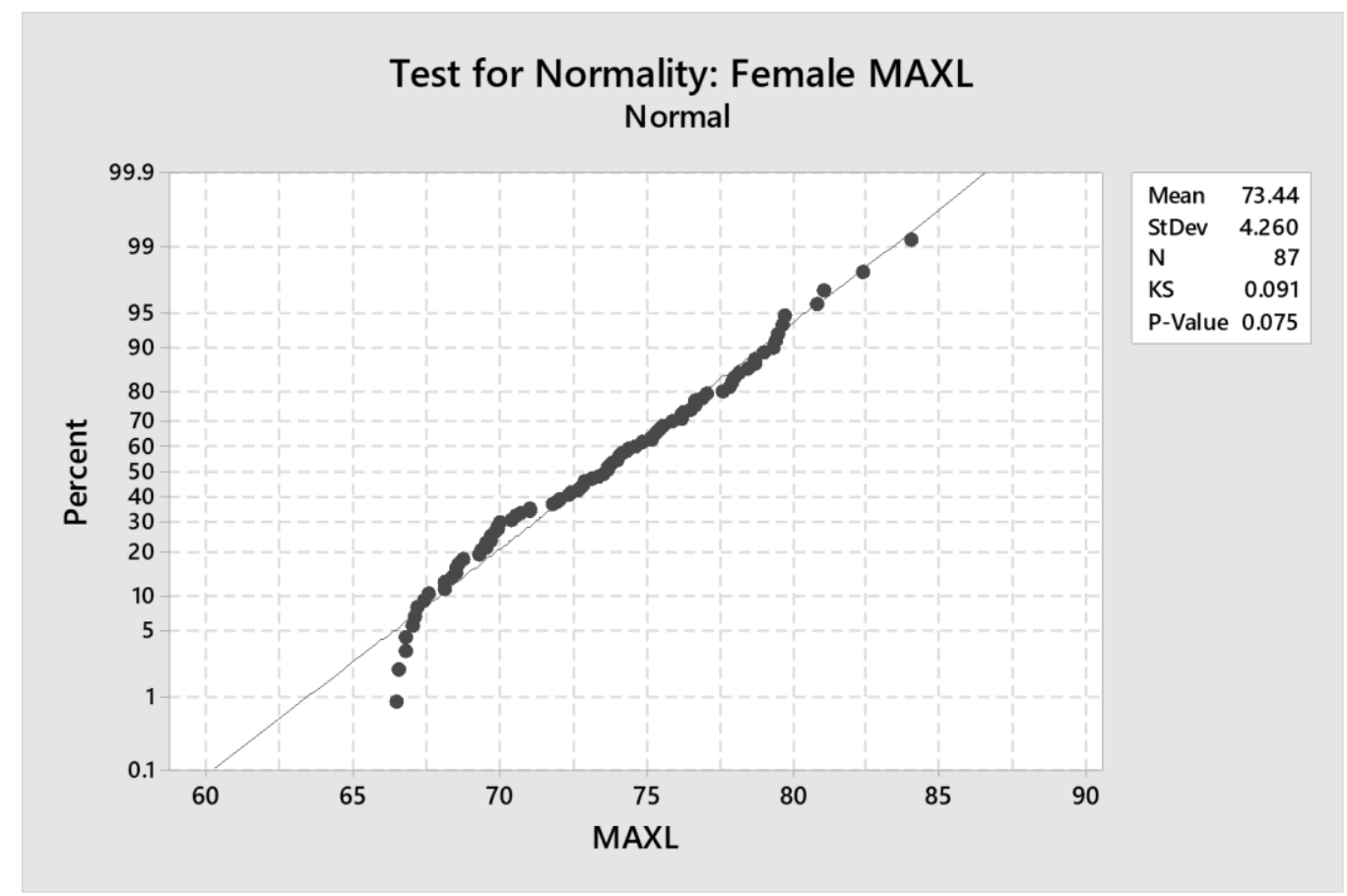

Appendix C2: Normality probability plot for female MAXL (Maximum Length) calcaneal measurements. 


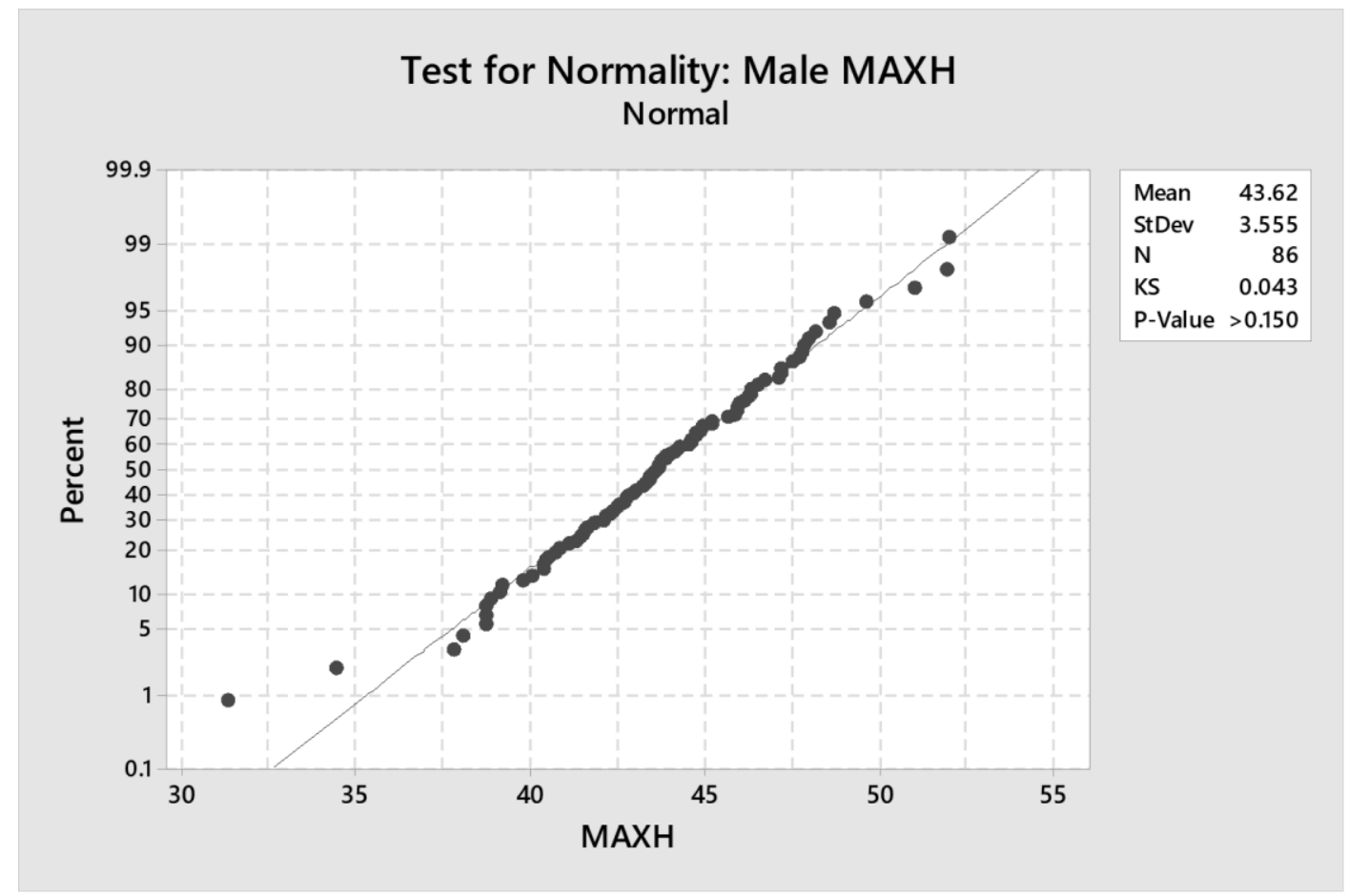

Appendix C3: Normality probability plot for male MAXH (Maximum Height) calcaneal measurements

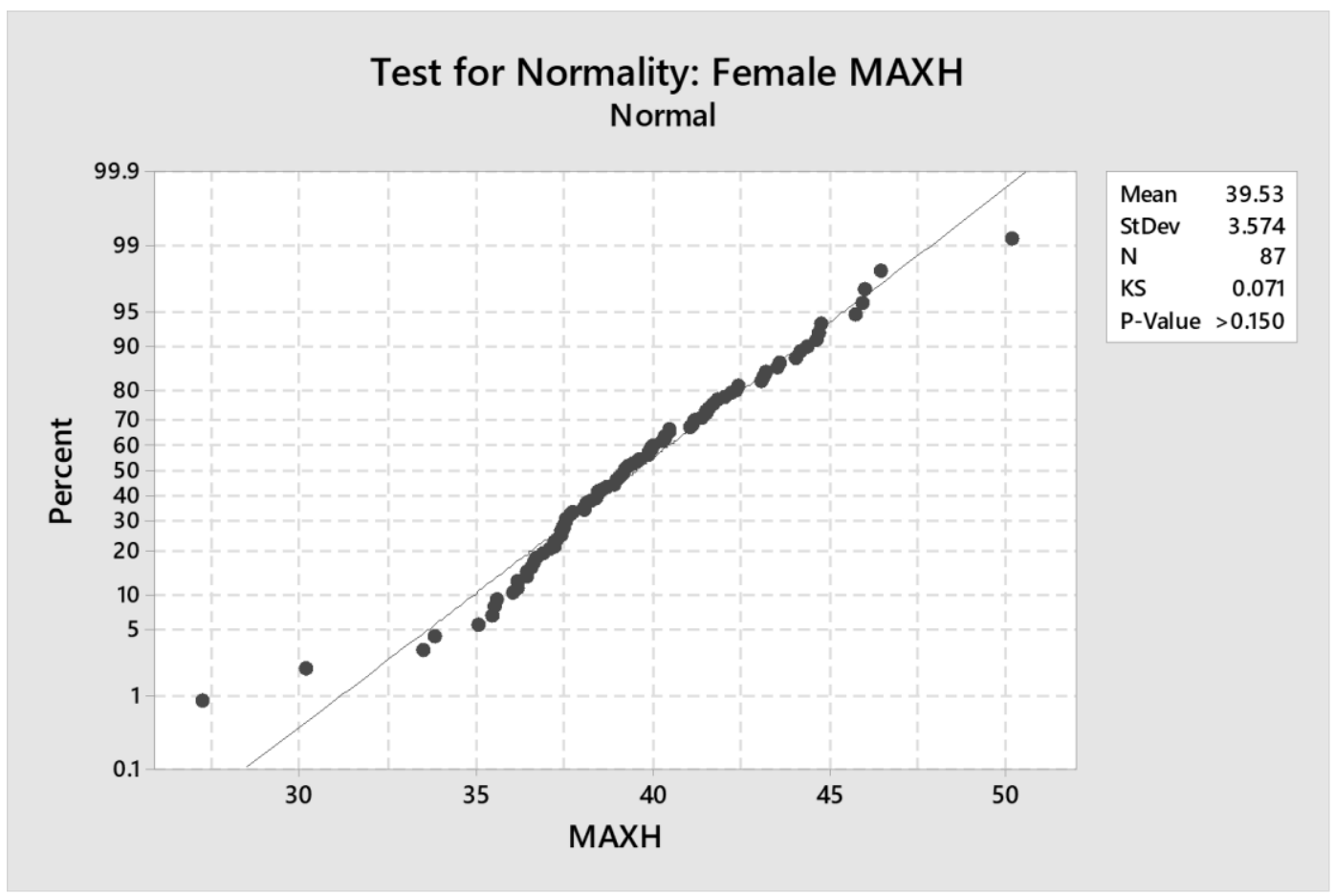

Appendix C4: Normality probability plot for female MAXH (Maximum Height) calcaneal measurements. 


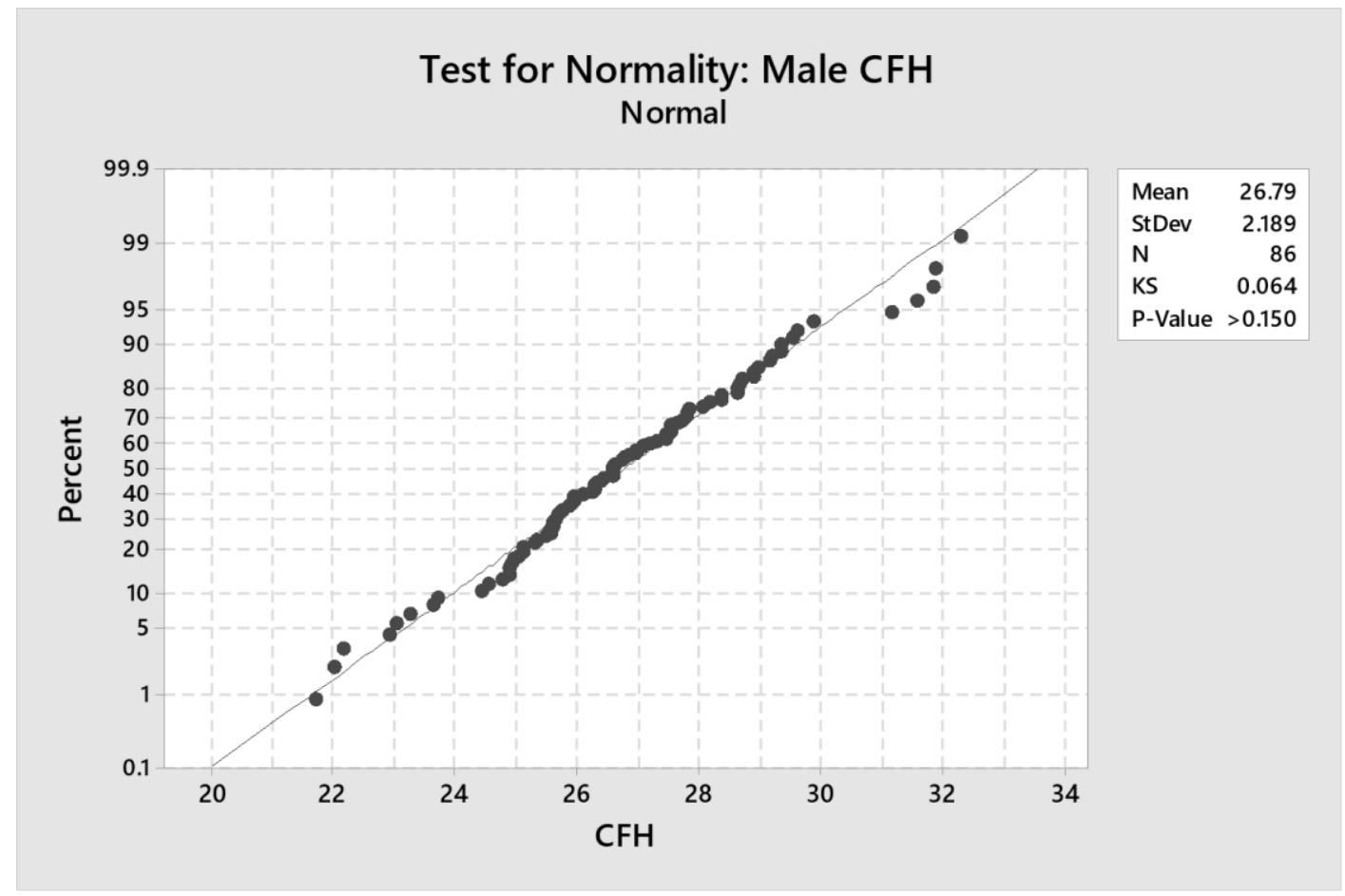

Appendix C5: Normality probability plot for male CFH (Cuboidal Facet Height) calcaneal measurements.

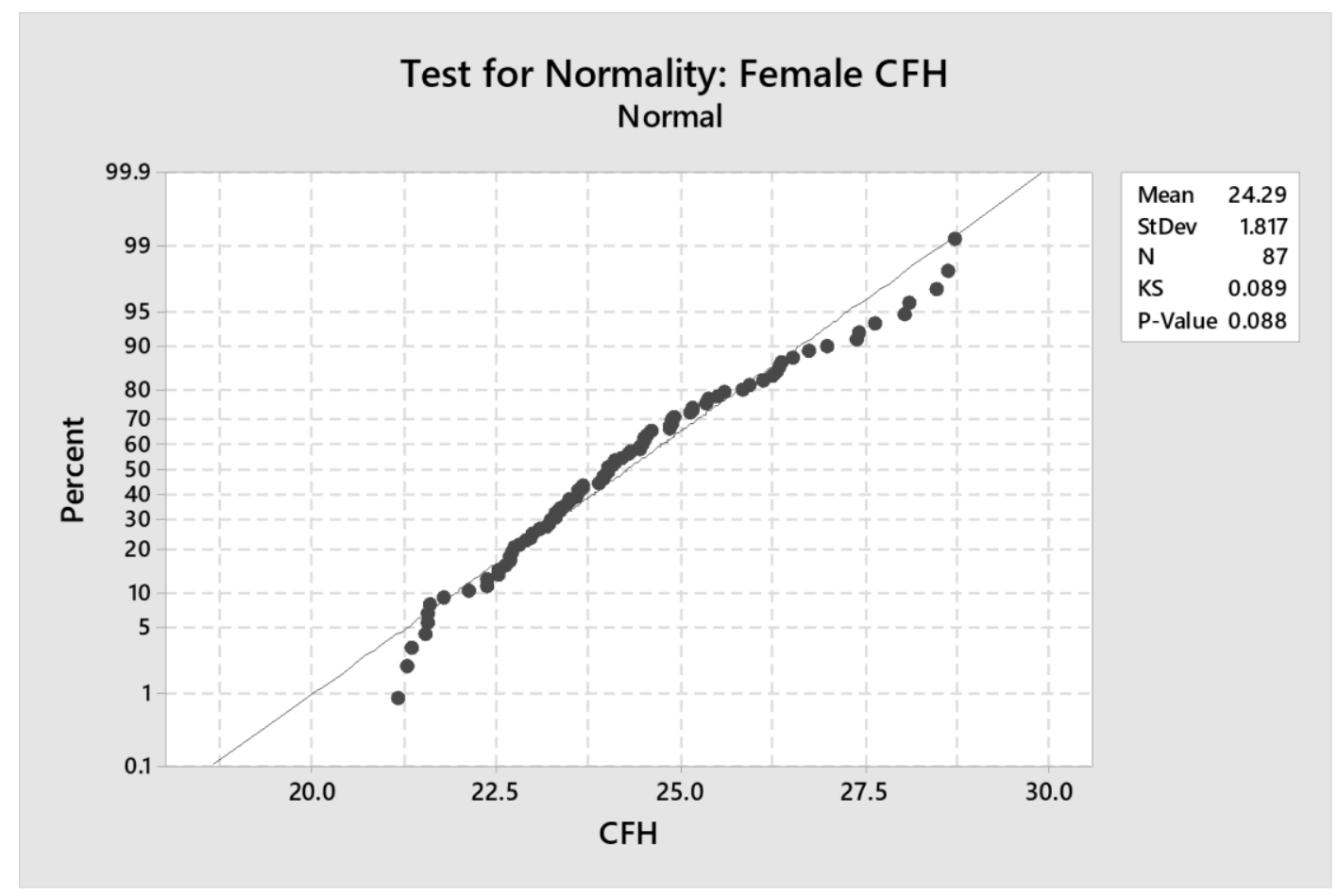

Appendix C6: Normality probability plot for female CFH (Cuboidal Facet Height) calcaneal measurements. 


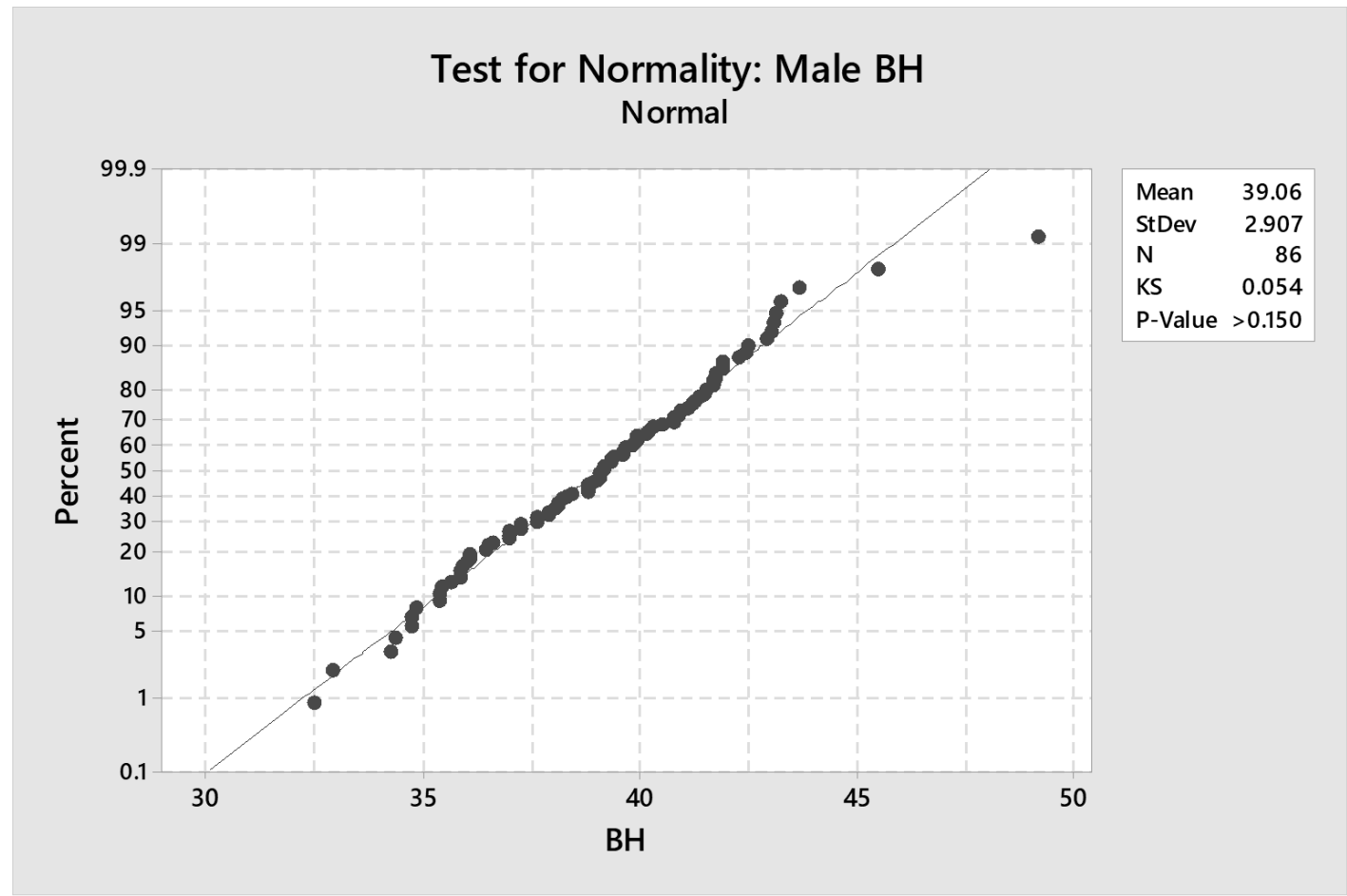

Appendix C7: Normality probability plot for male BH (Body Height) calcaneal measurements

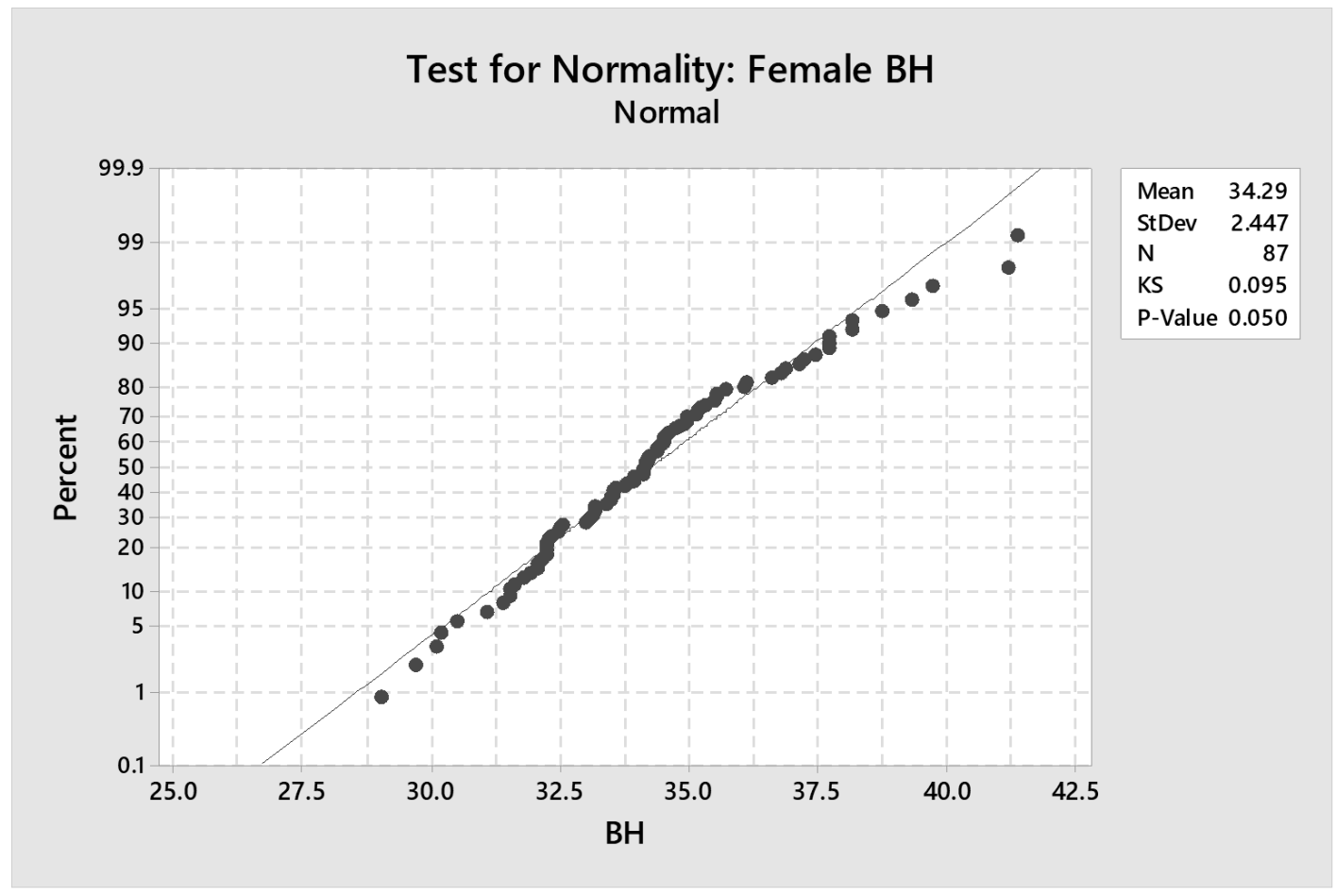

Appendix C8: Normality probability plot for female BH (Body Height) calcaneal measurements 


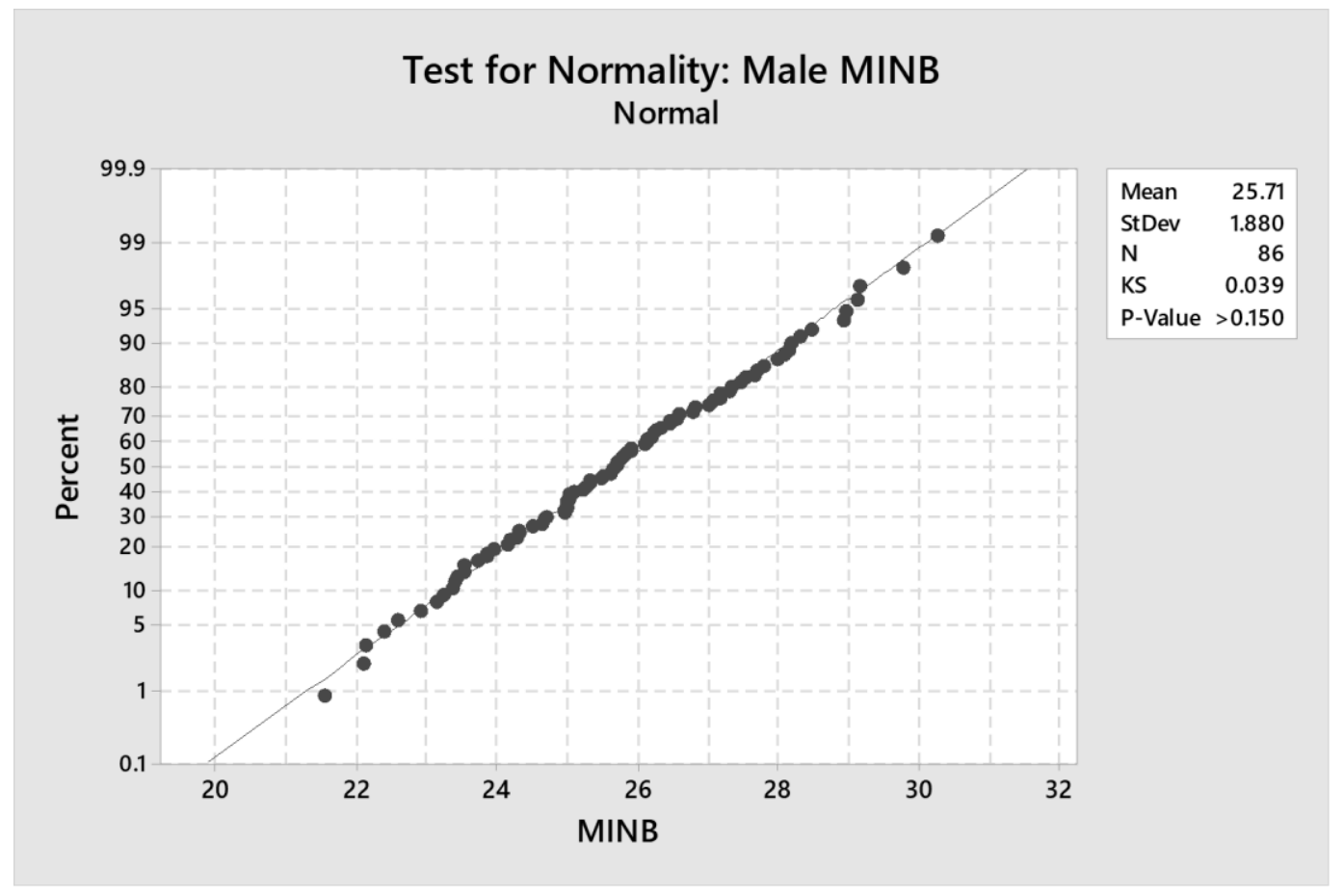

Appendix C9: Normality probability plot for male MINB (Minimum Breadth) calcaneal measurements

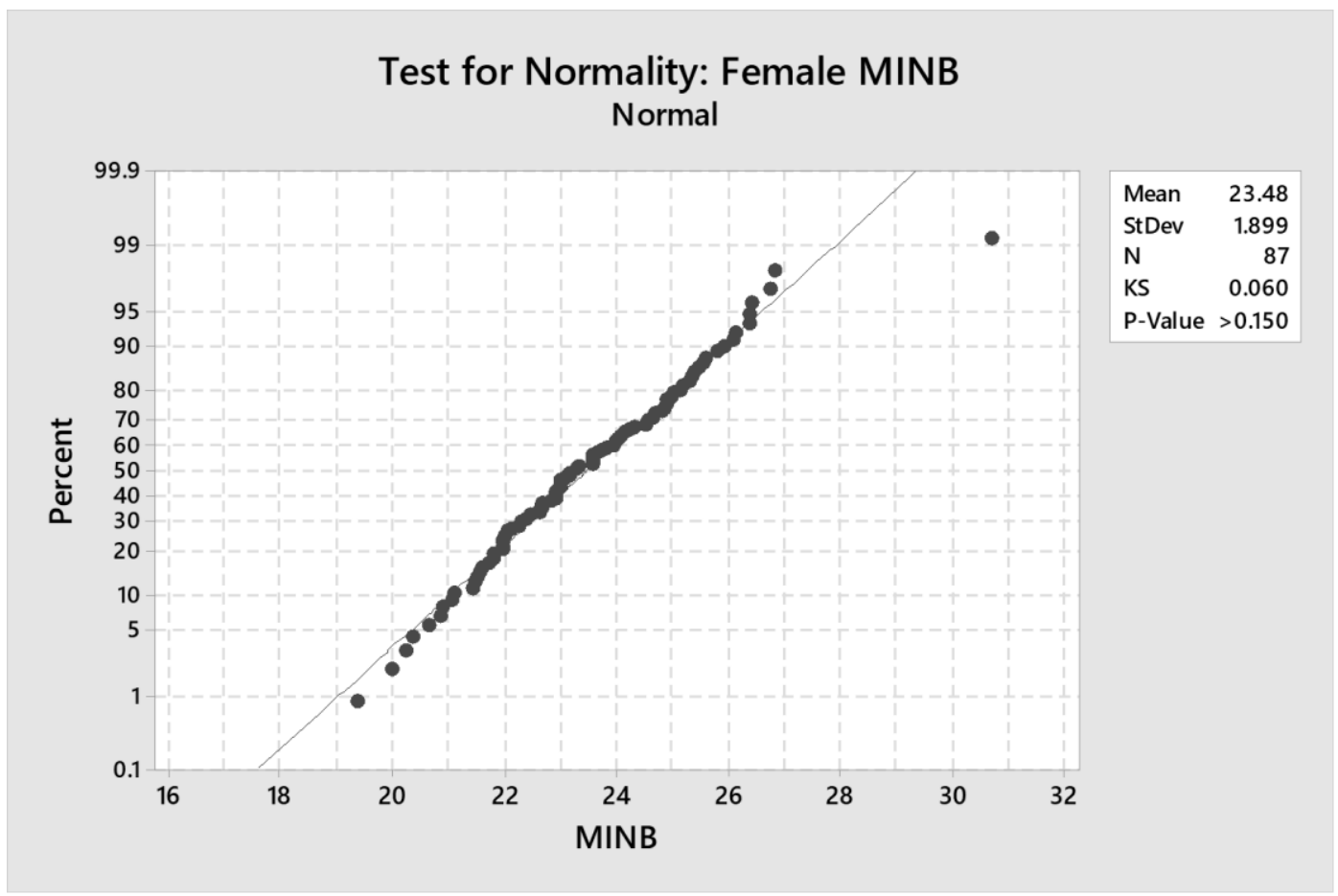

Appendix C10: Normality probability plot for female MINB (Minimum Breadth) calcaneal measurements 


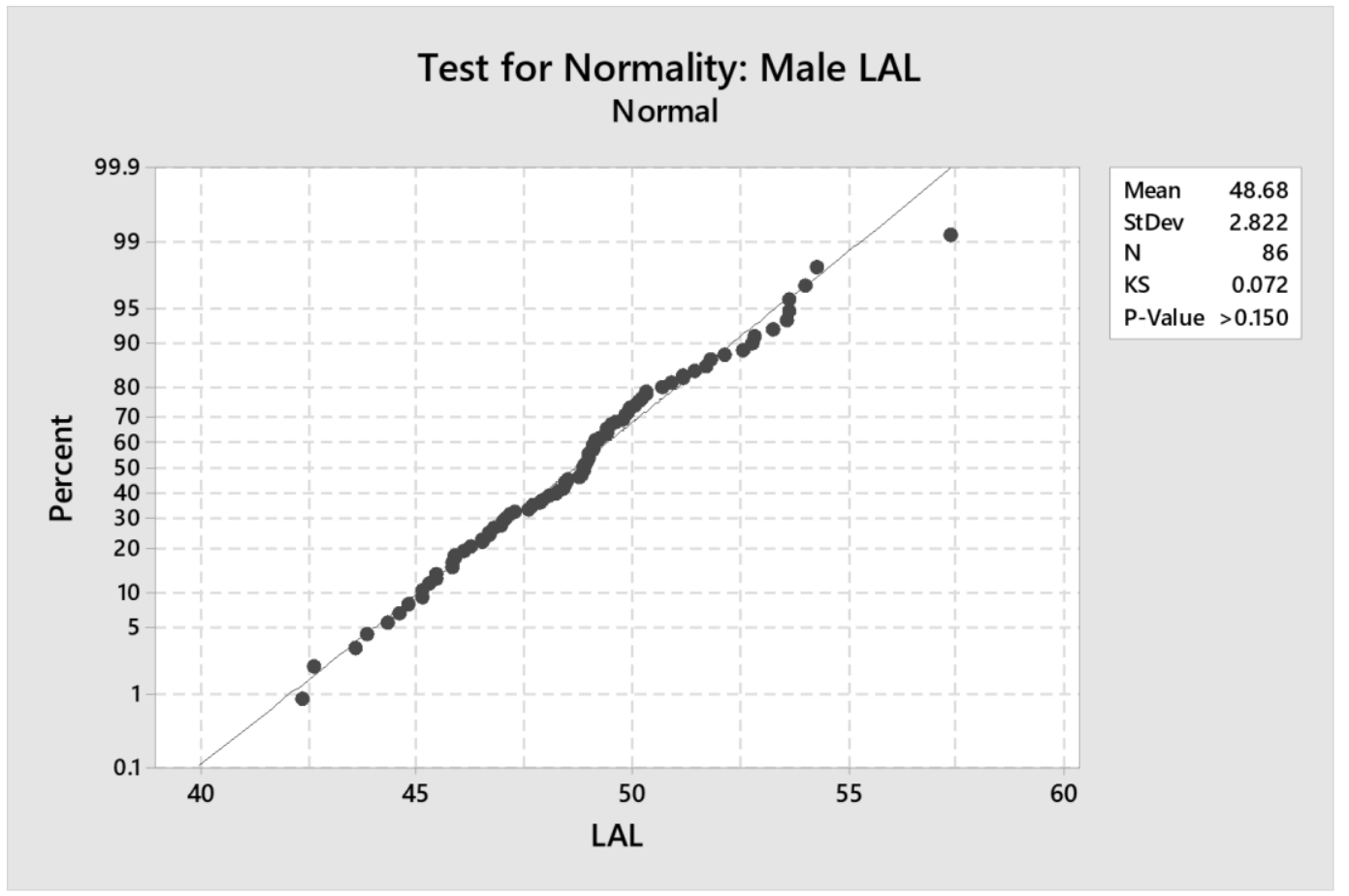

Appendix C11: Normality probability plot for male LAL (Load Arm Length) calcaneal measurements

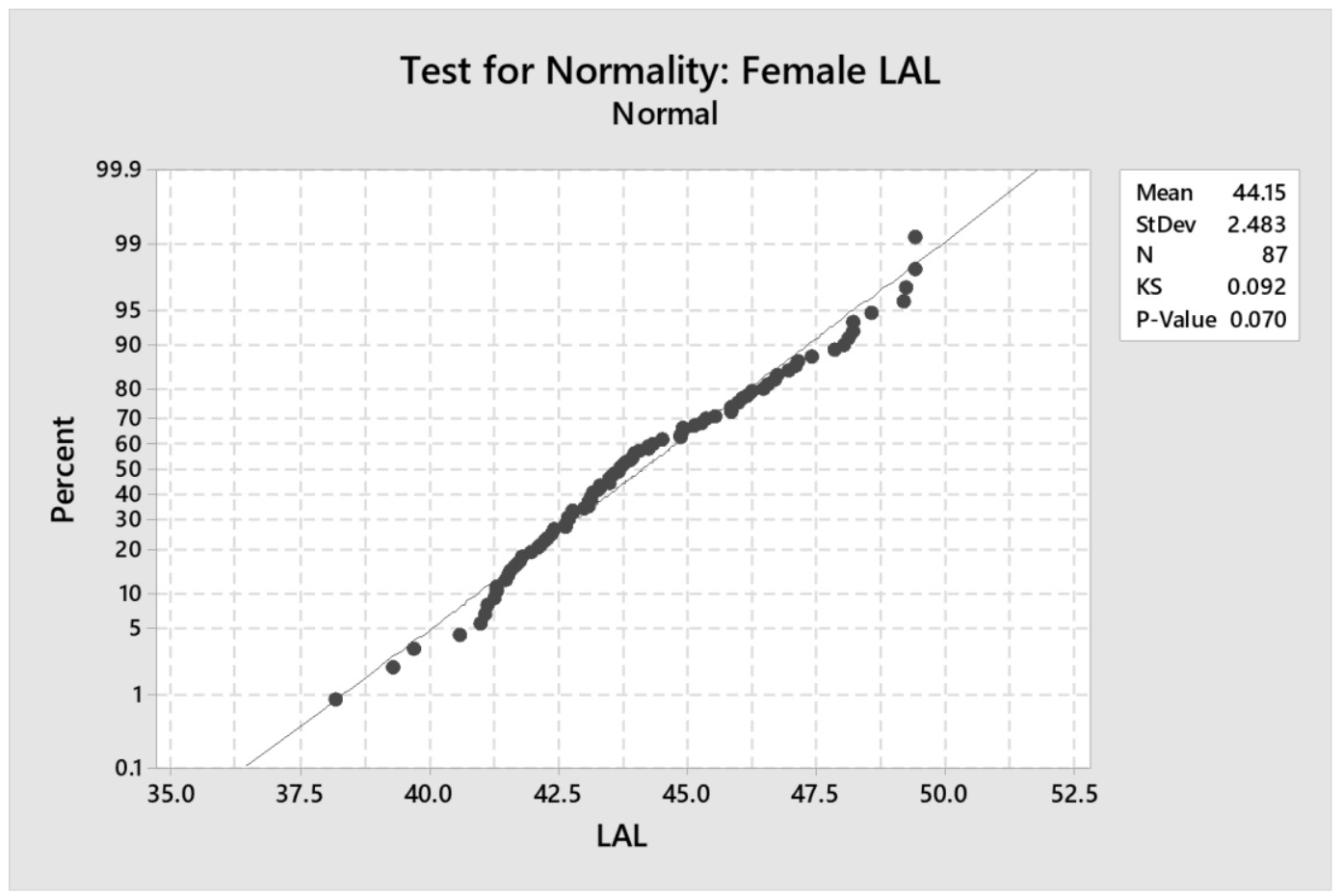

Appendix C12: Normality probability plot for female LAL (Load Arm Length) calcaneal measurements 


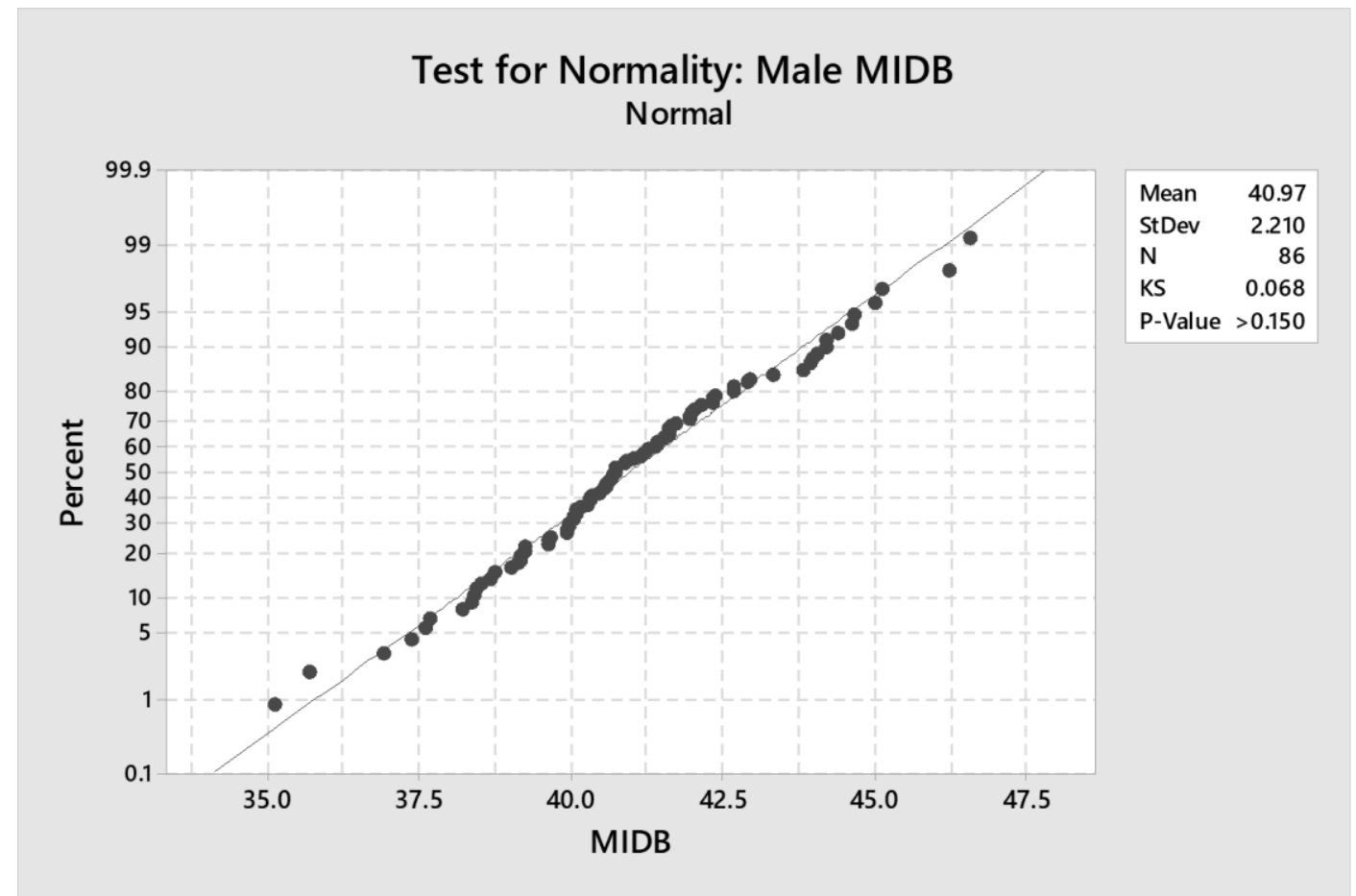

Appendix C13: Normality probability plot for male MIDB (Middle Breadth) calcaneal measurements

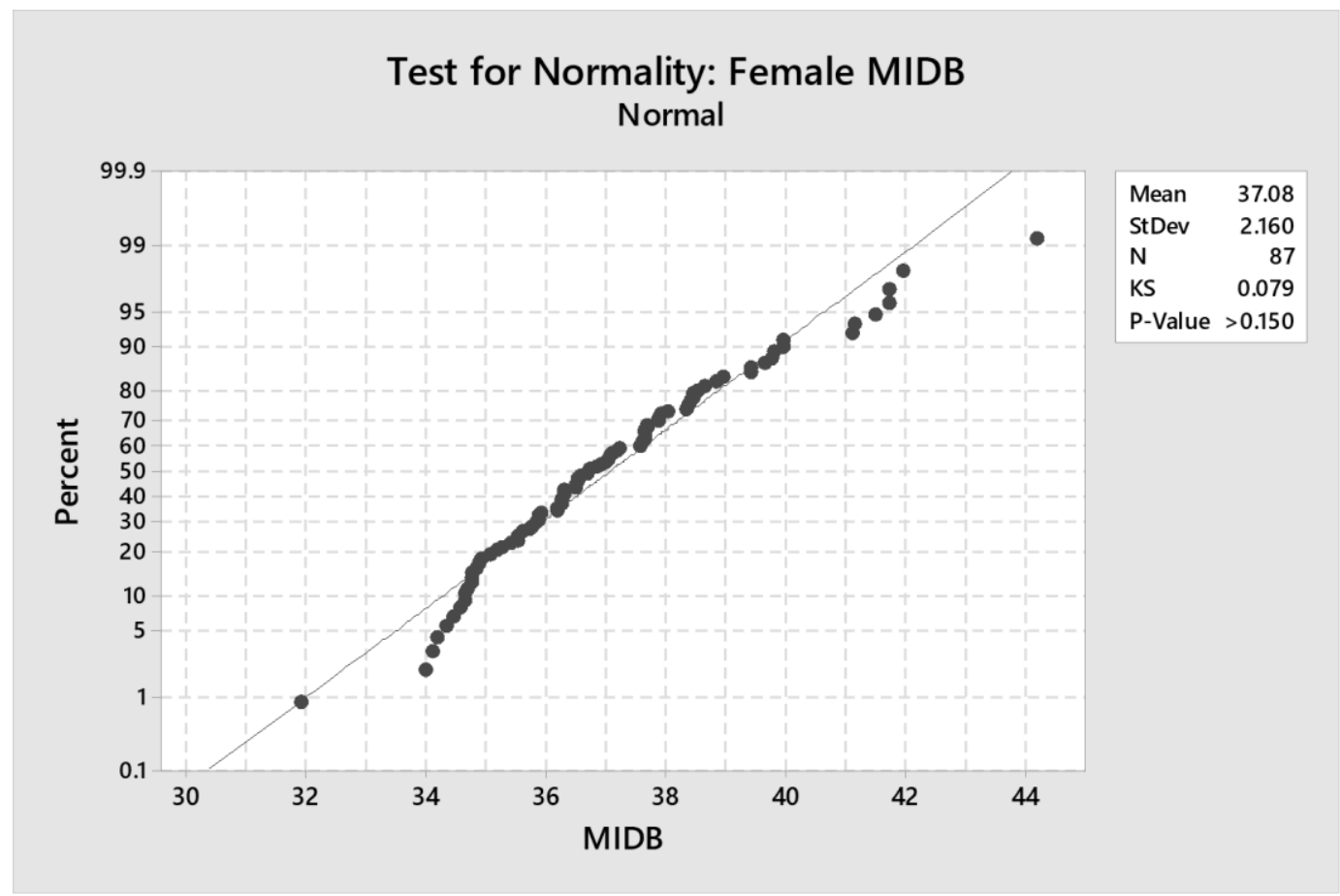

Appendix C14: Normality probability plot for female MIDB (Middle Breadth) calcaneal measurements 


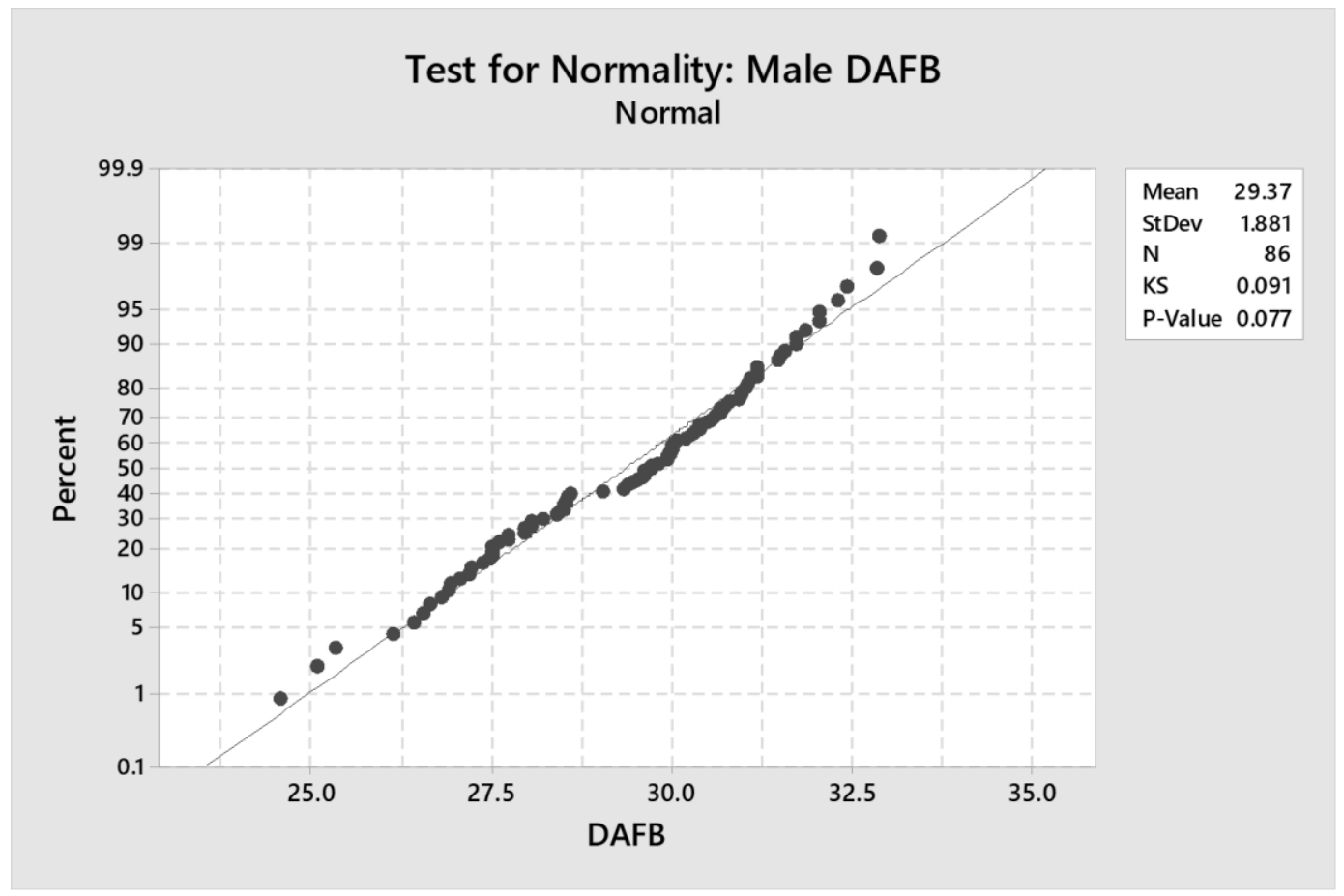

Appendix C15: Normality probability plot for male DAFB (Dorsal Articular Facet Breadth) calcaneal measurements

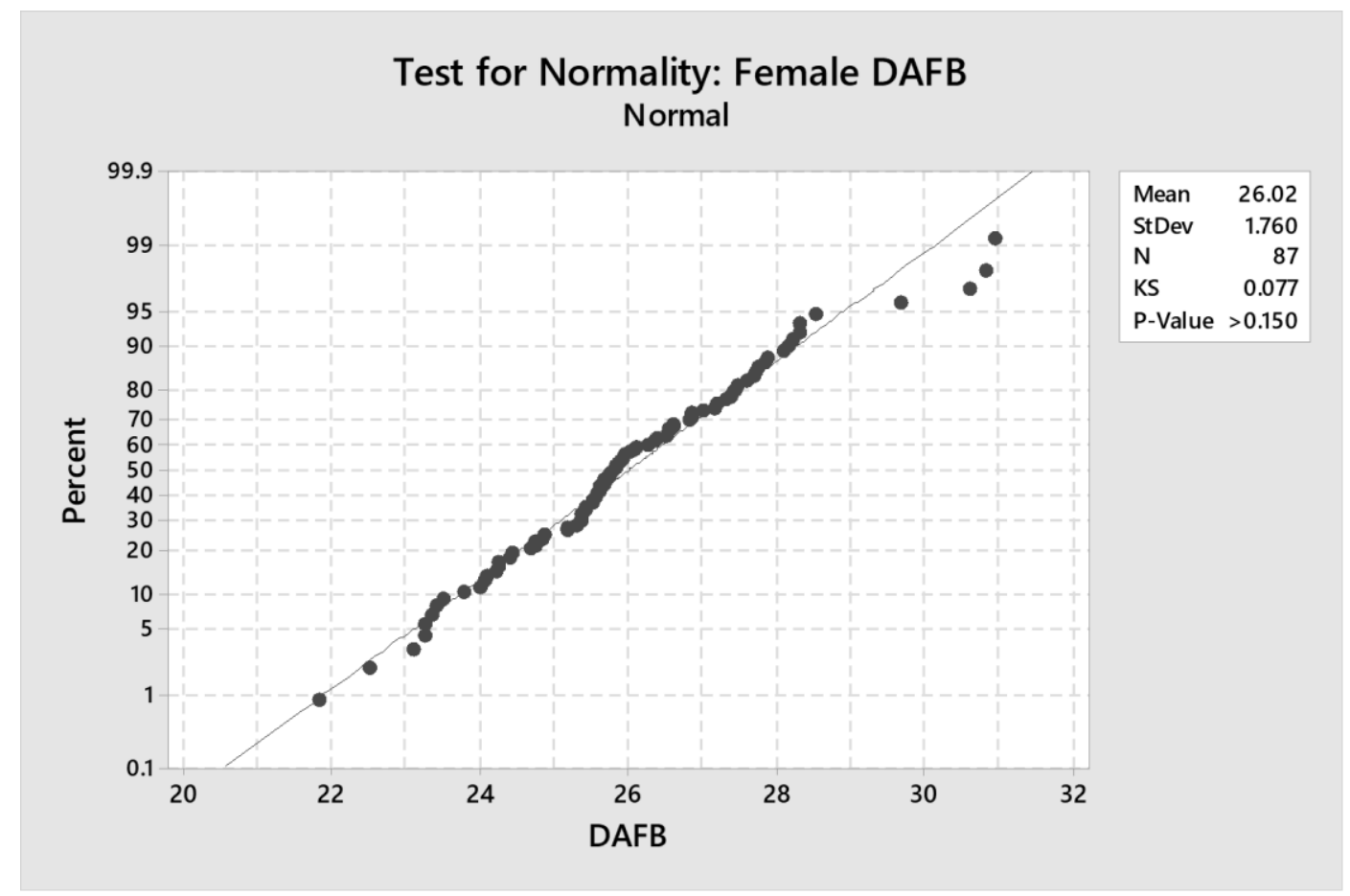

Appendix C16: Normality probability plot for female DAFB (Dorsal Articular Facet Breadth) calcaneal measurements 


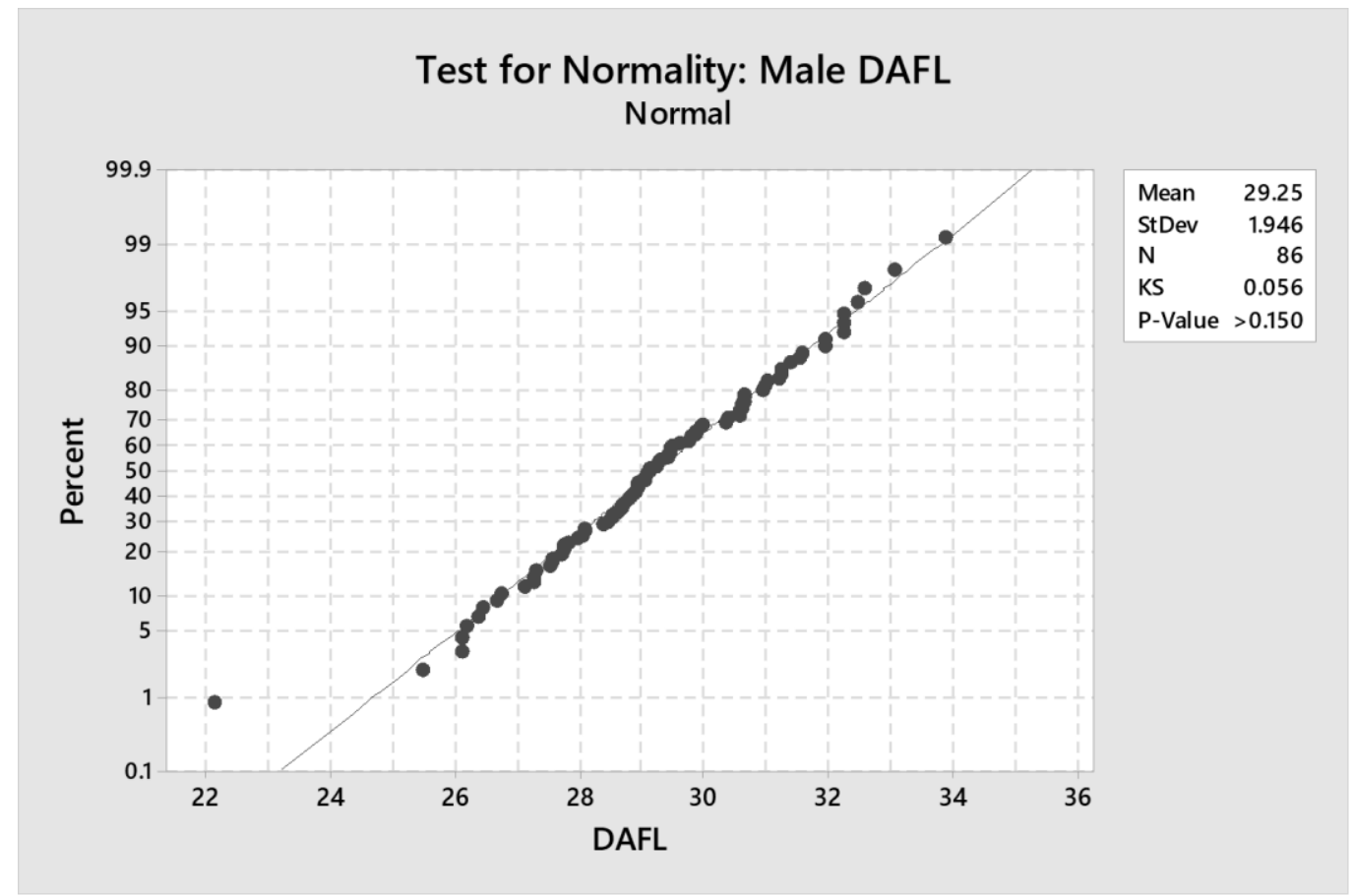

Appendix C17: Normality probability plot for male DAFL (Dorsal Articular Facet Length) calcaneal measurements

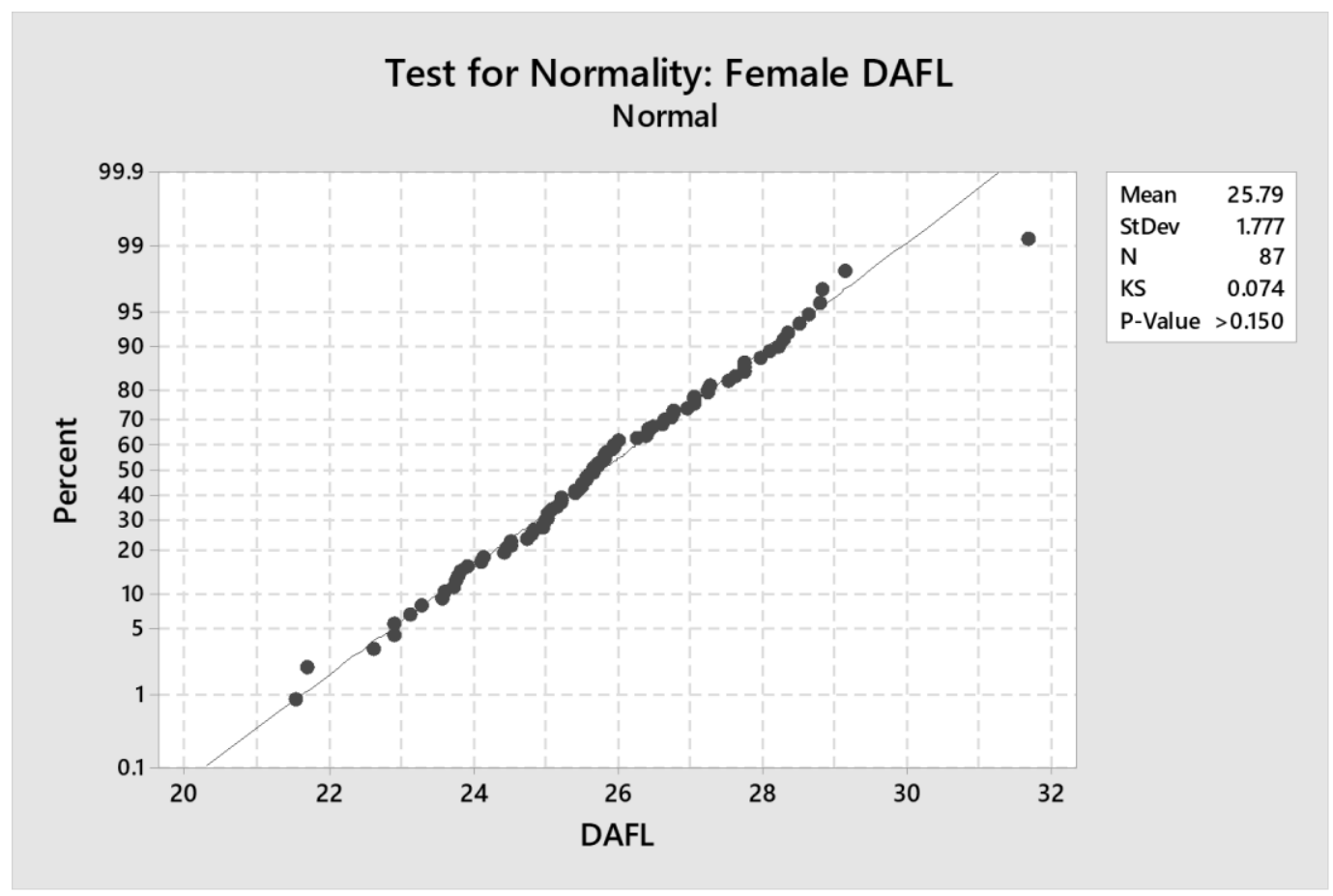

Appendix C18: Normality probability plot for female DAFL (Dorsal Articular Facet Length) calcaneal measurements 


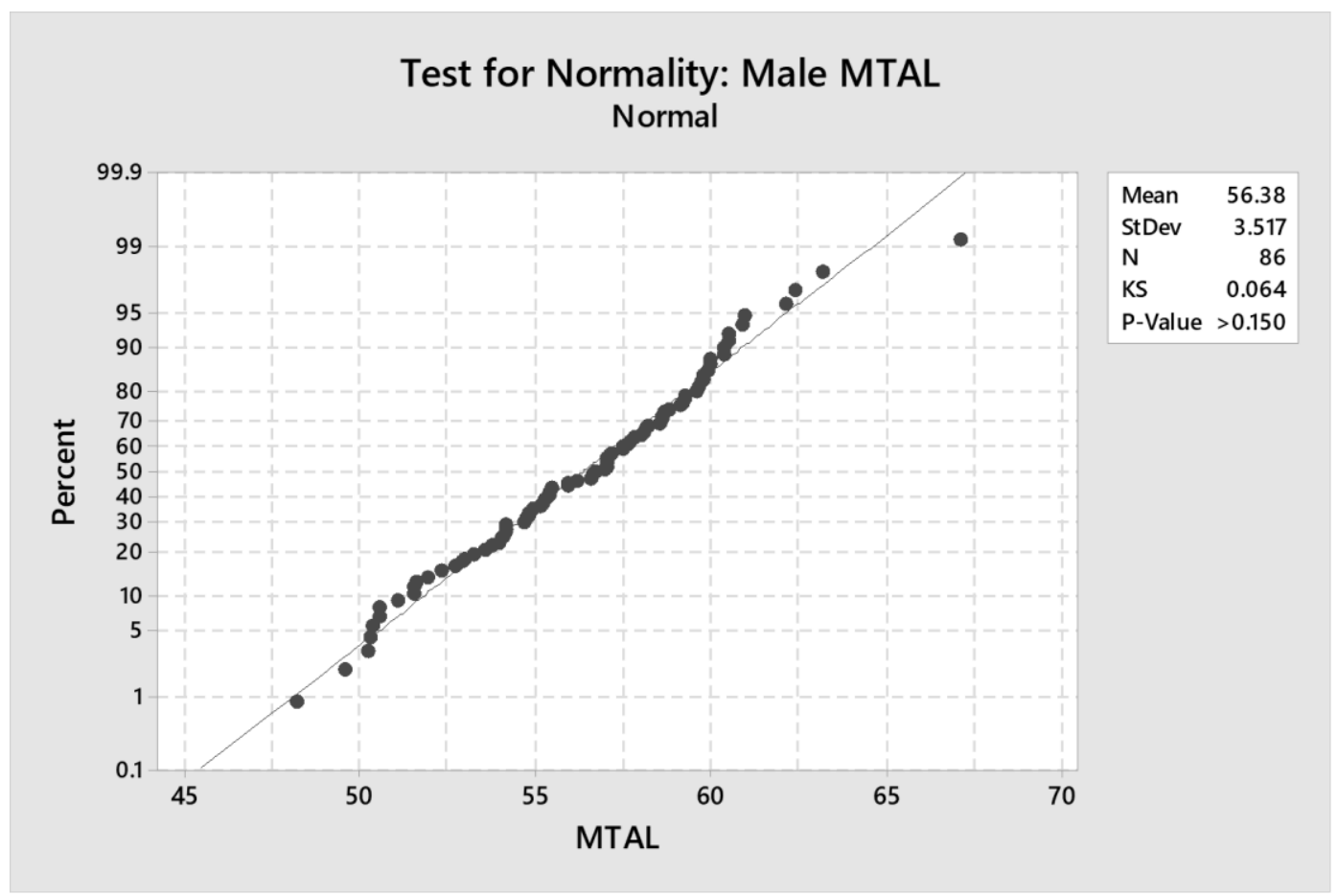

Appendix C19: Normality probability plot for male MTAL (Maximum Length of the Talus) talar measurements

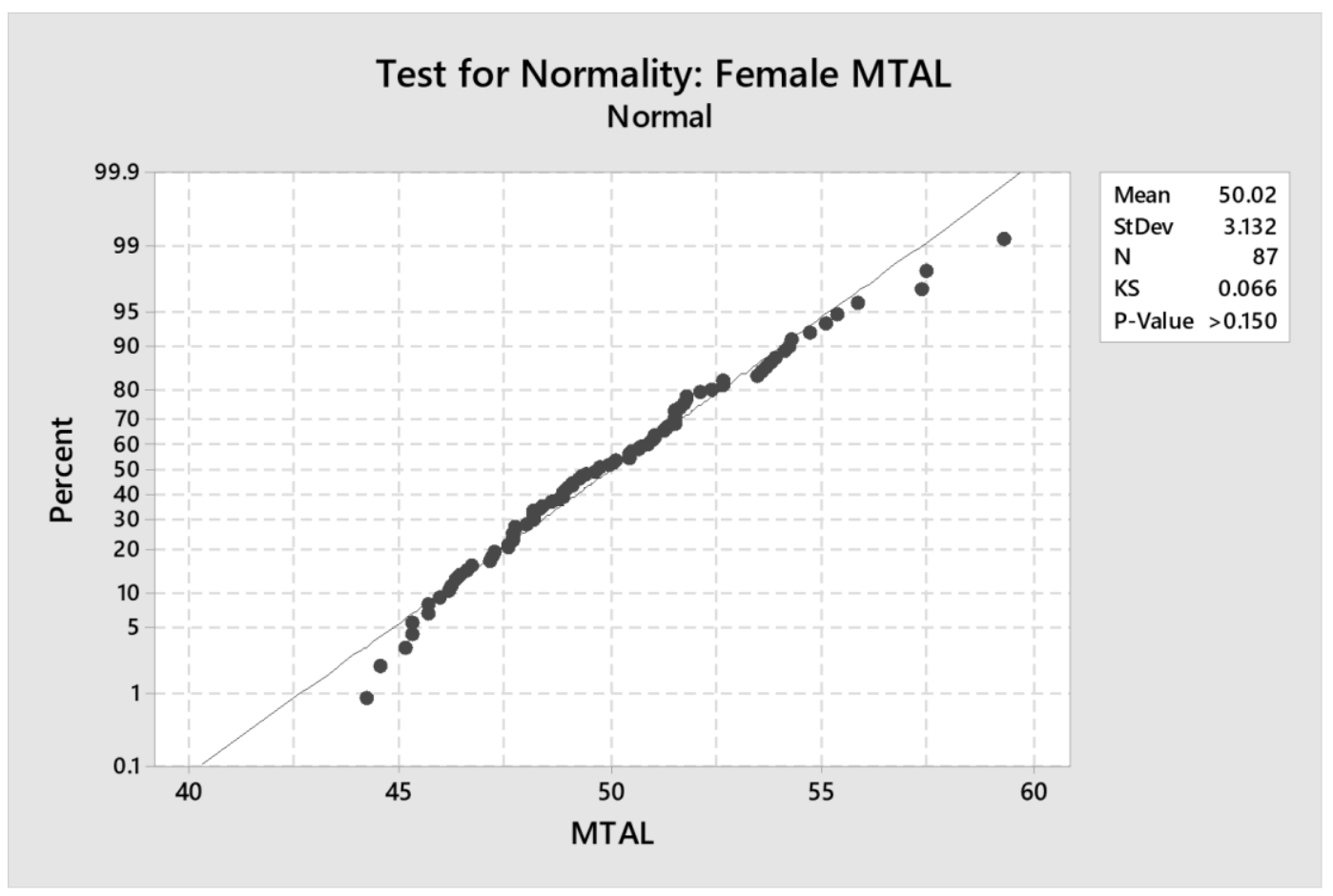

Appendix C20: Normality probability plot for female MTAL (Maximum Length of the Talus) talar measurements 
APPENDIX D:

ACCURACIES OF OTHER POPULATIONS SIMPLE AND MULTIPLE REGRESSION EQUATIONS WHEN APPLIED TO THE THAI POPULATION 


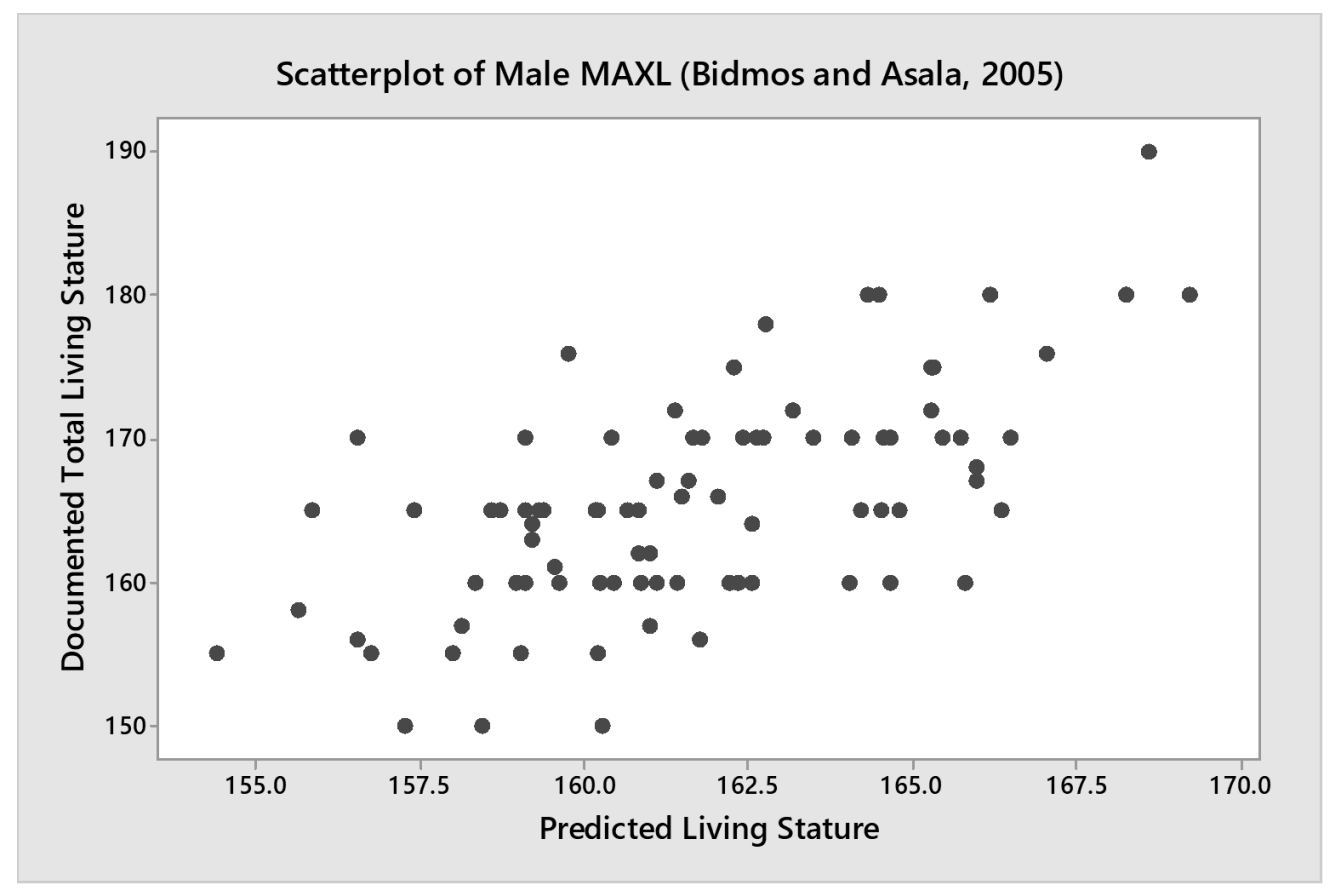

Appendix D1: Simple scatterplot showing the correlation between documented living stature and the predicted living stature, using the male Black South African (Bidmos and Asala, 2005) MAXL simple regression equation.

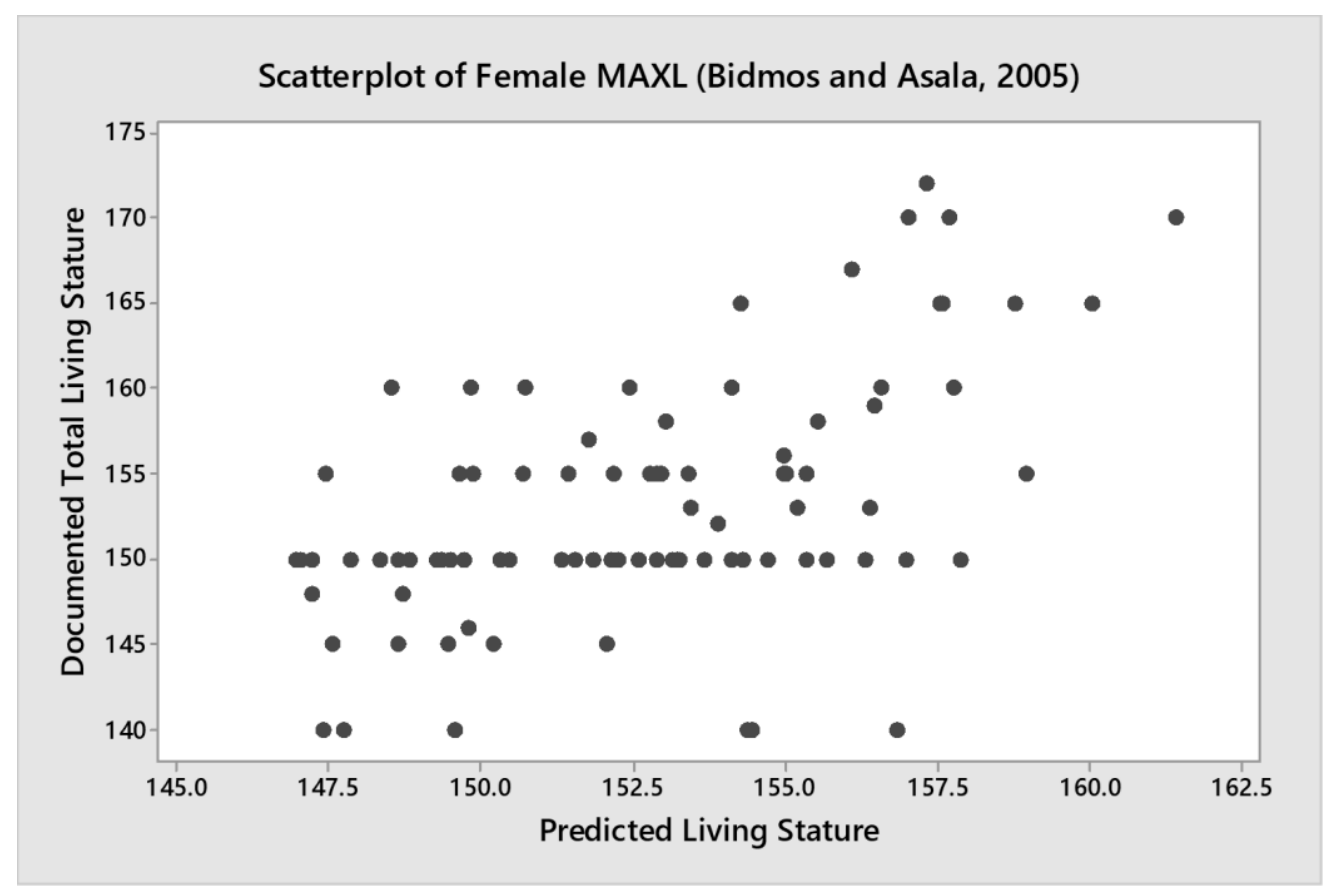

Appendix D2: Simple scatterplot showing the correlation between documented living stature and the predicted living stature, using the female Black South African (Bidmos and Asala, 2005) MAXL simple regression equation. 


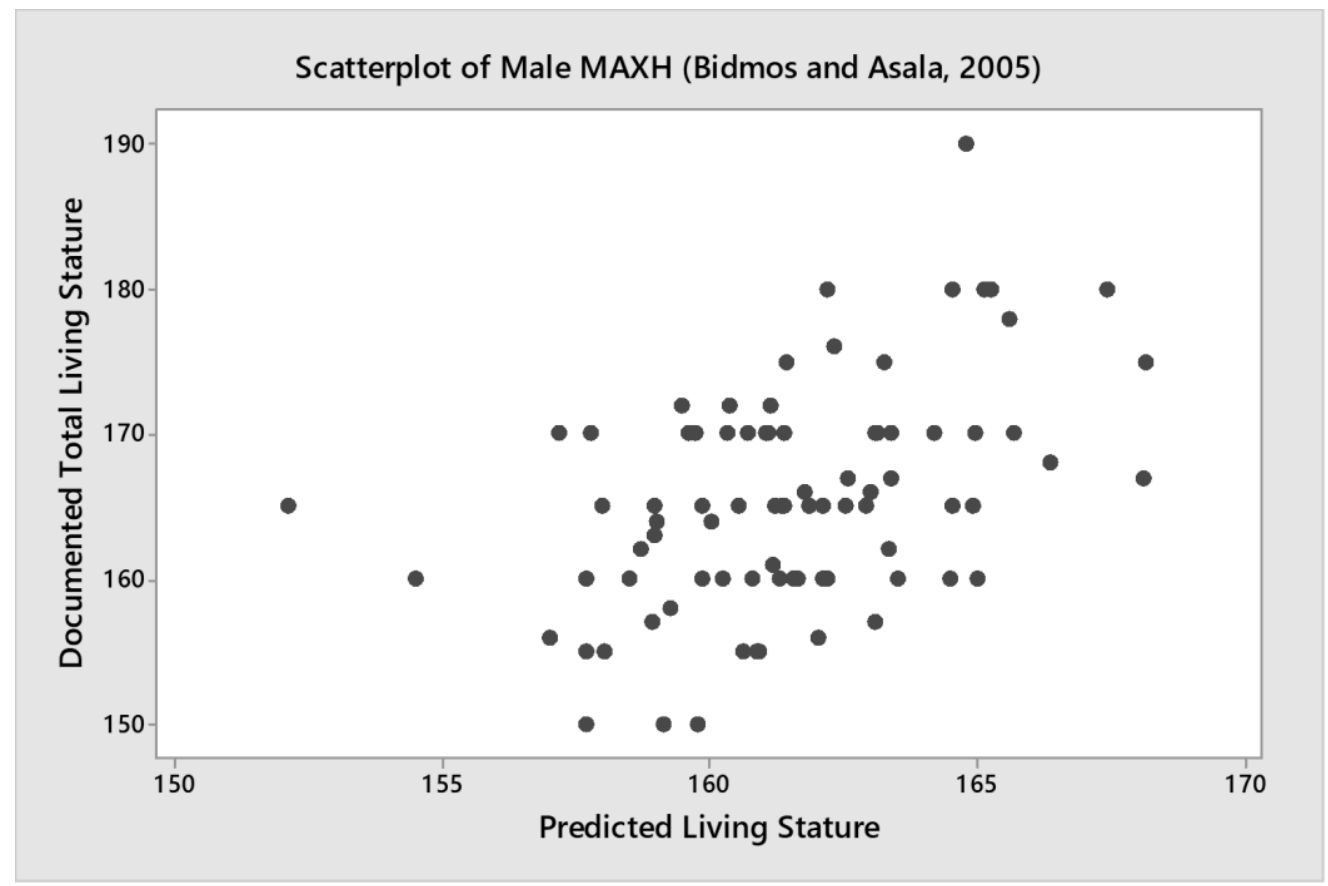

Appendix D3: Simple scatterplot showing the correlation between documented living stature and the predicted living stature, using the male Black South African (Bidmos and Asala, 2005) MAXH simple regression equation.

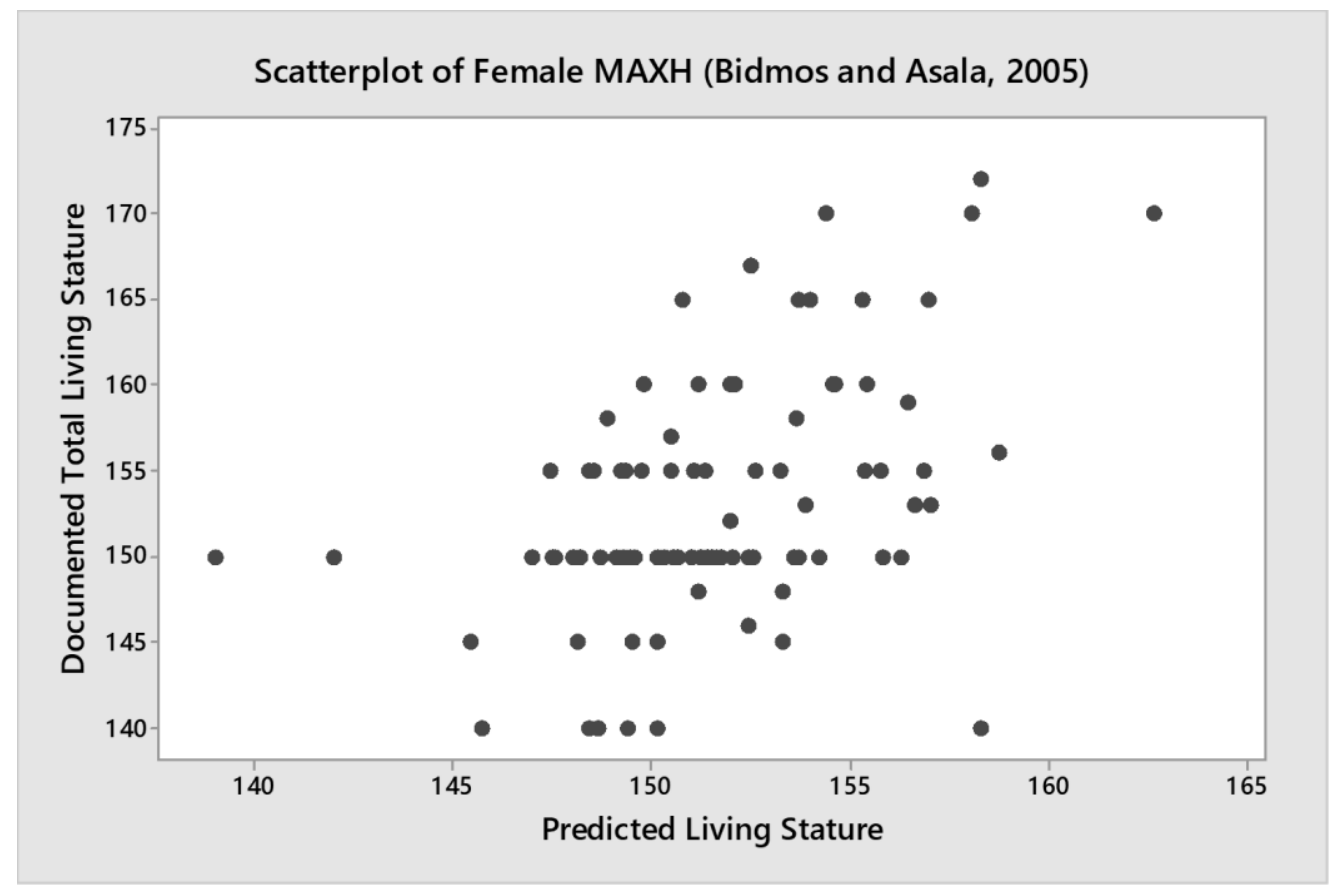

Appendix D4: Simple scatterplot showing the correlation between documented living stature and the predicted living stature, using the female Black South African (Bidmos and Asala, 2005) MAXH simple regression equation. 


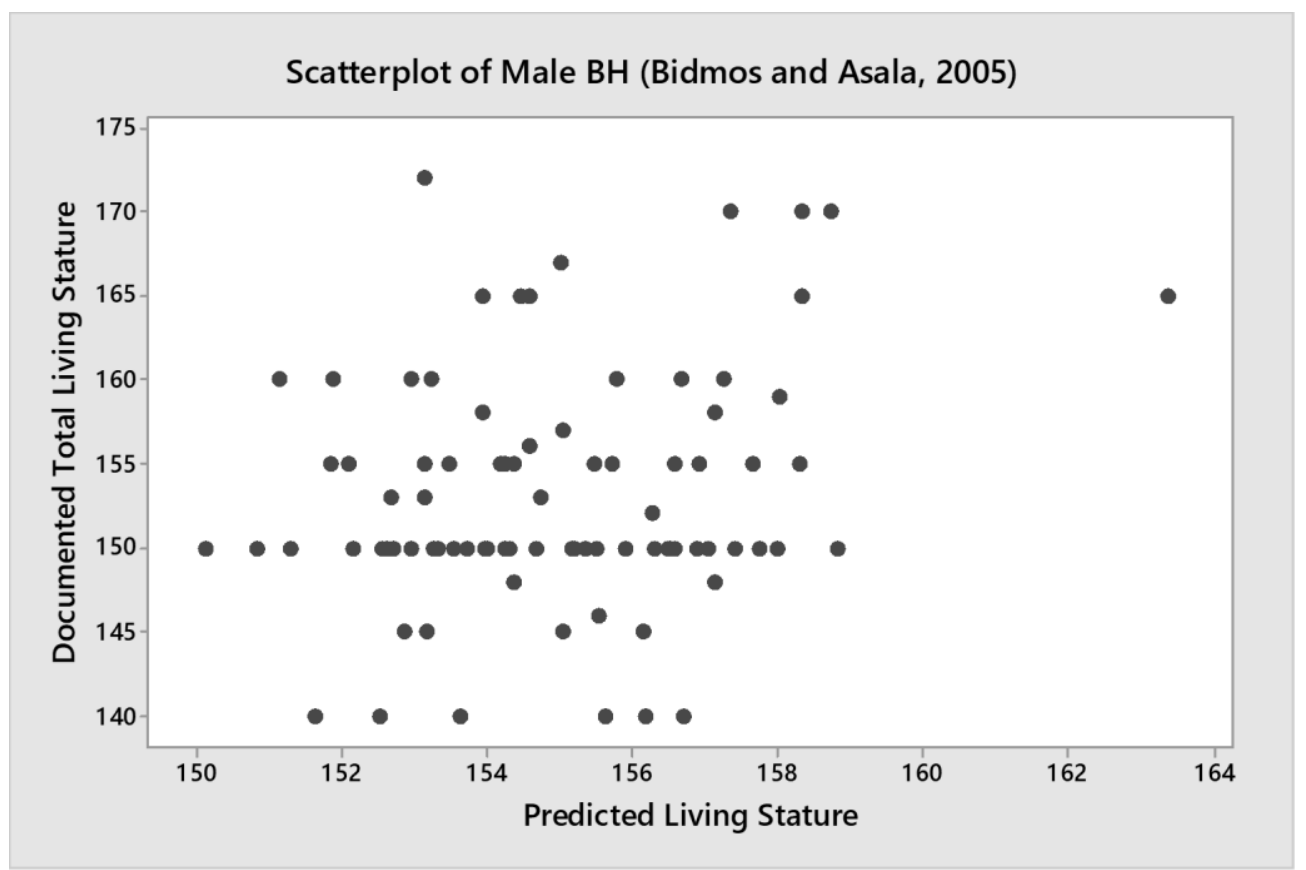

Appendix D5: Simple scatterplot showing the correlation between documented living stature and the predicted living stature, using the male Black South African (Bidmos and Asala, 2005) BH simple regression equation.

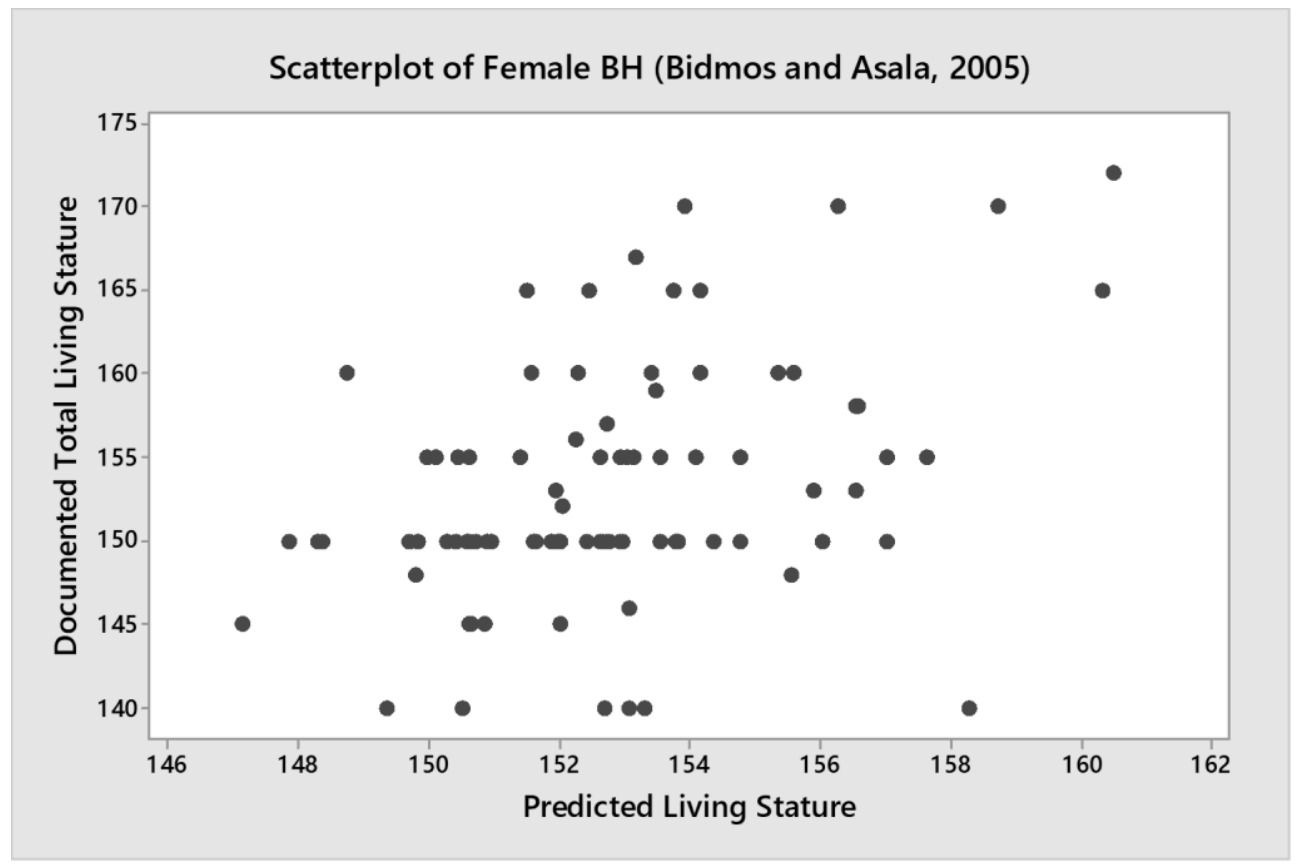

Appendix D6: Simple scatterplot showing the correlation between documented living stature and the predicted living stature, using the female Black South African (Bidmos and Asala, 2005) BH simple regression equation. 


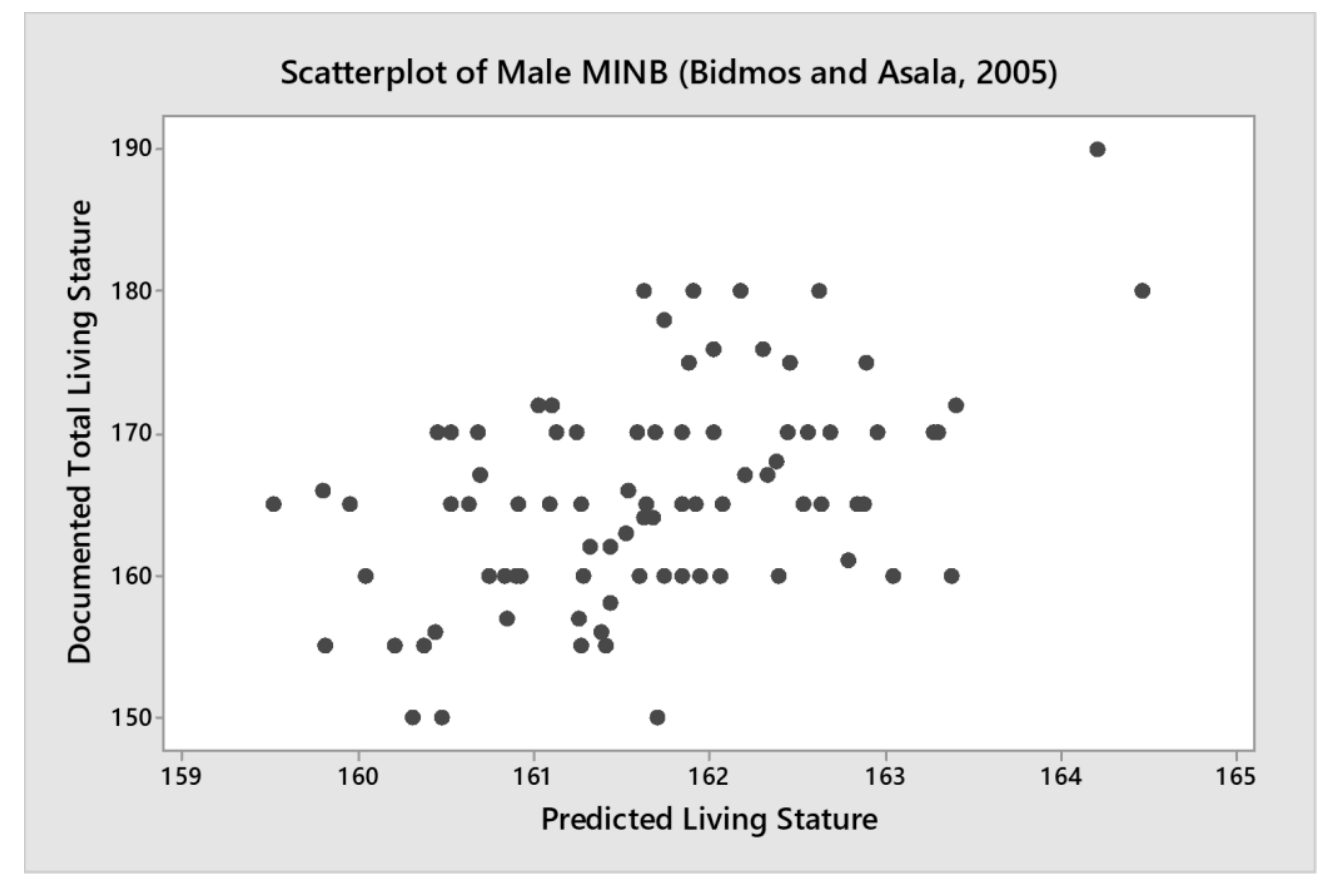

Appendix D7: Simple scatterplot showing the correlation between documented living stature and the predicted living stature, using the male Black South African (Bidmos and Asala, 2005) MINB simple regression equation.

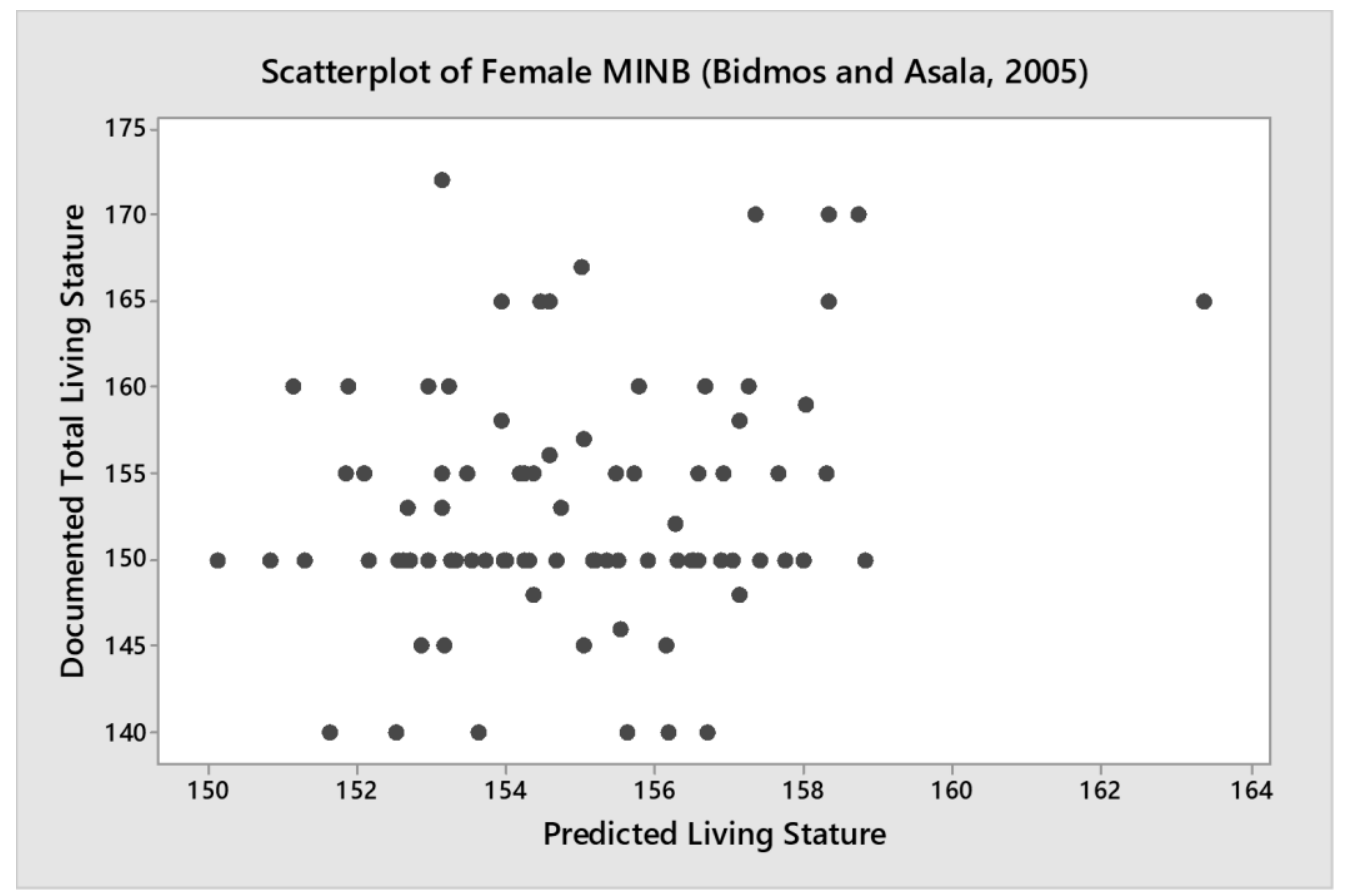

Appendix D8: Simple scatterplot showing the correlation between documented living stature and the predicted living stature, using the female Black South African (Bidmos and Asala, 2005) MINB simple regression equation. 


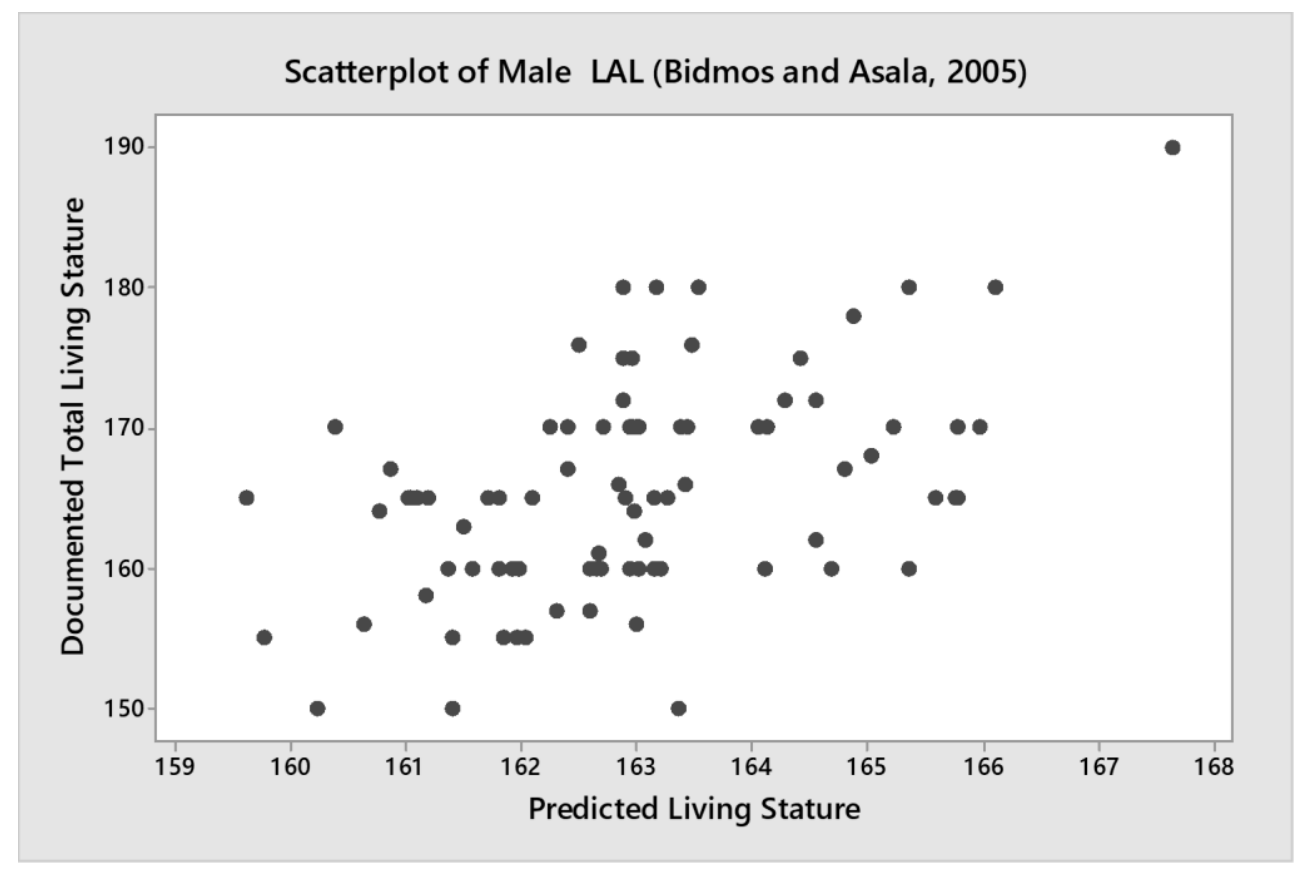

Appendix D9: Simple scatterplot showing the correlation between documented living stature and the predicted living stature, using the male Black South African (Bidmos and Asala, 2005) LAL simple regression equation.

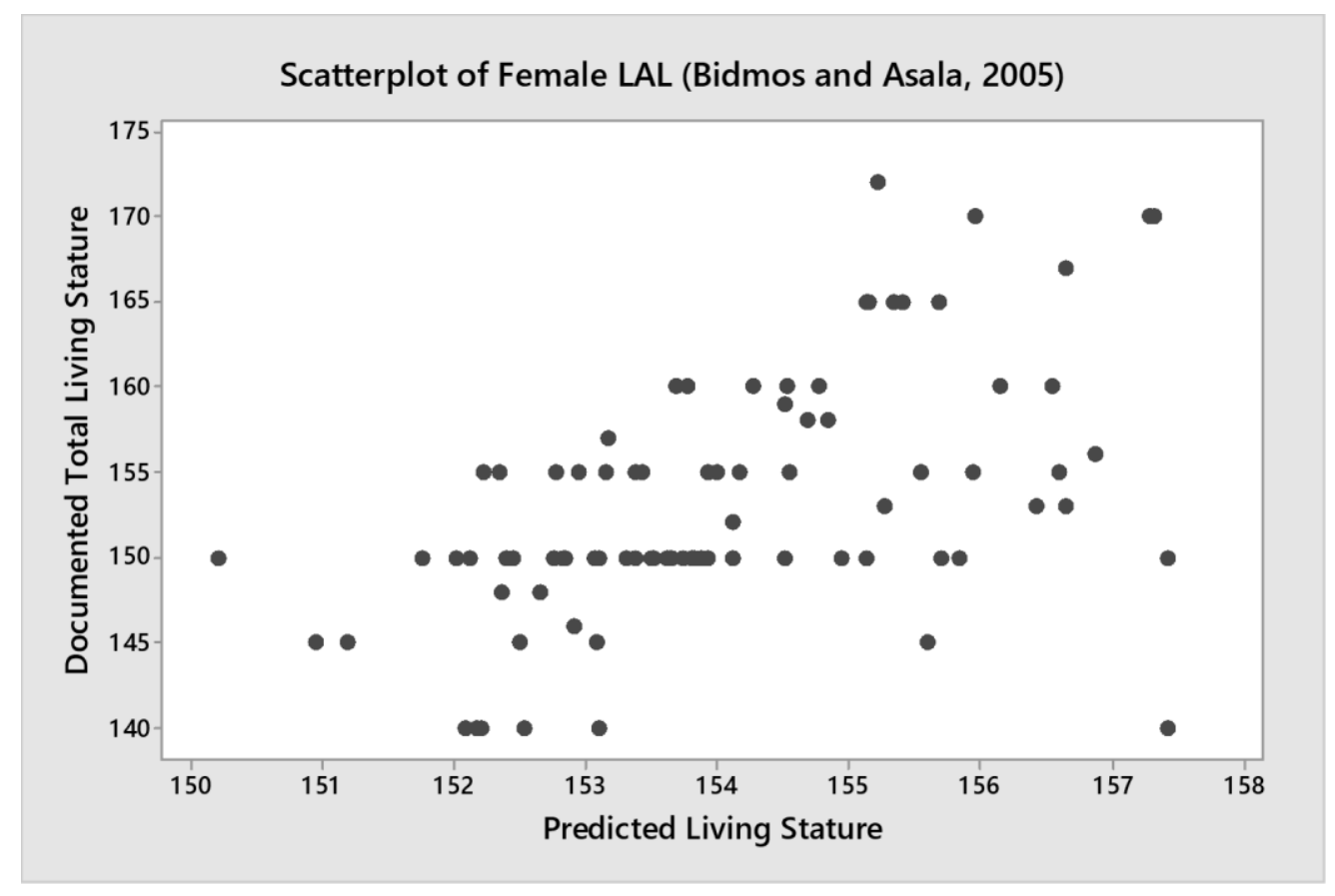

Appendix D10: Simple scatterplot showing the correlation between documented living stature and the predicted living stature, using the female Black South African (Bidmos and Asala, 2005) LAL simple regression equation. 


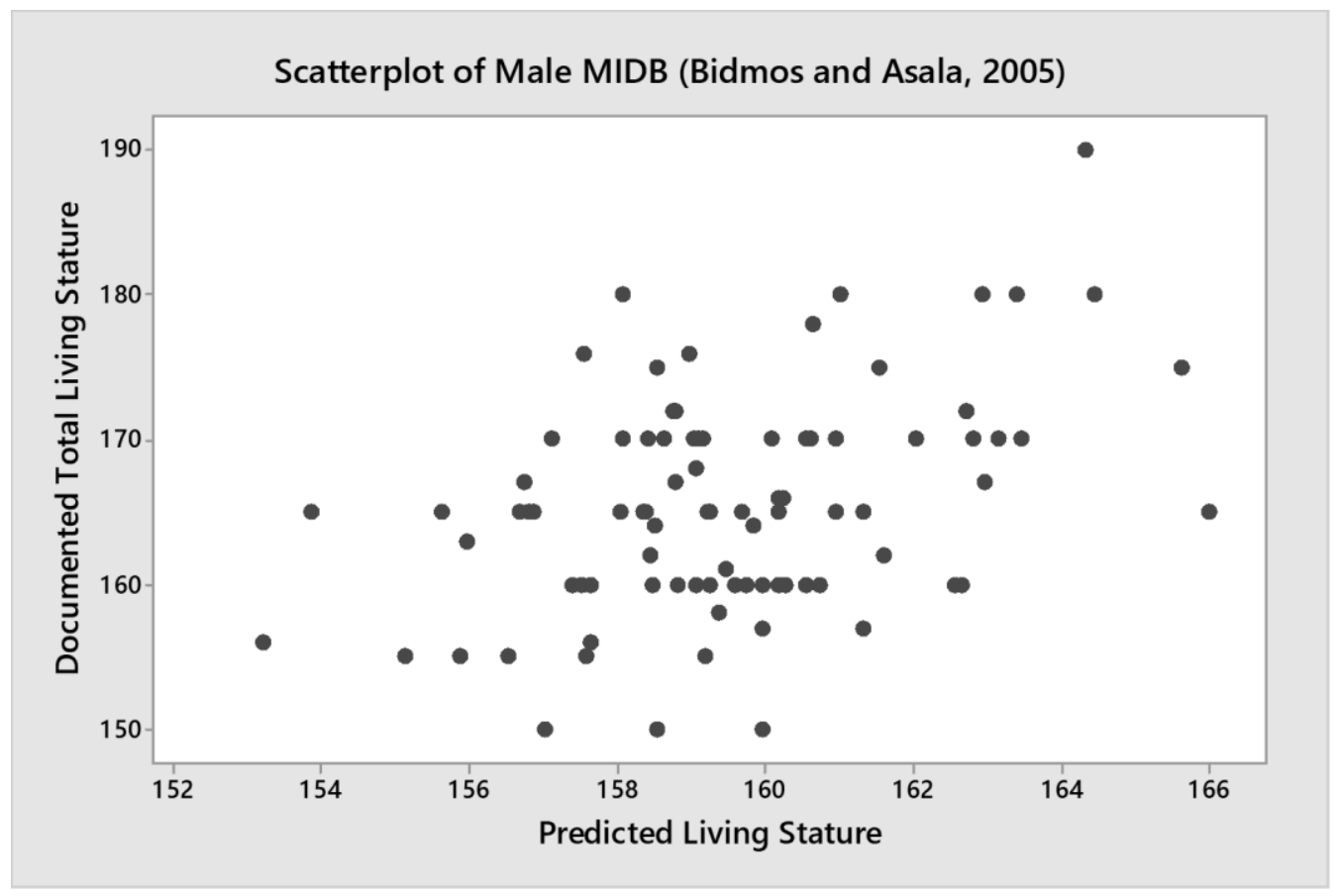

Appendix D11: Simple scatterplot showing the correlation between documented living stature and the predicted living stature, using the male Black South African (Bidmos and Asala, 2005) MIDB simple regression equation.

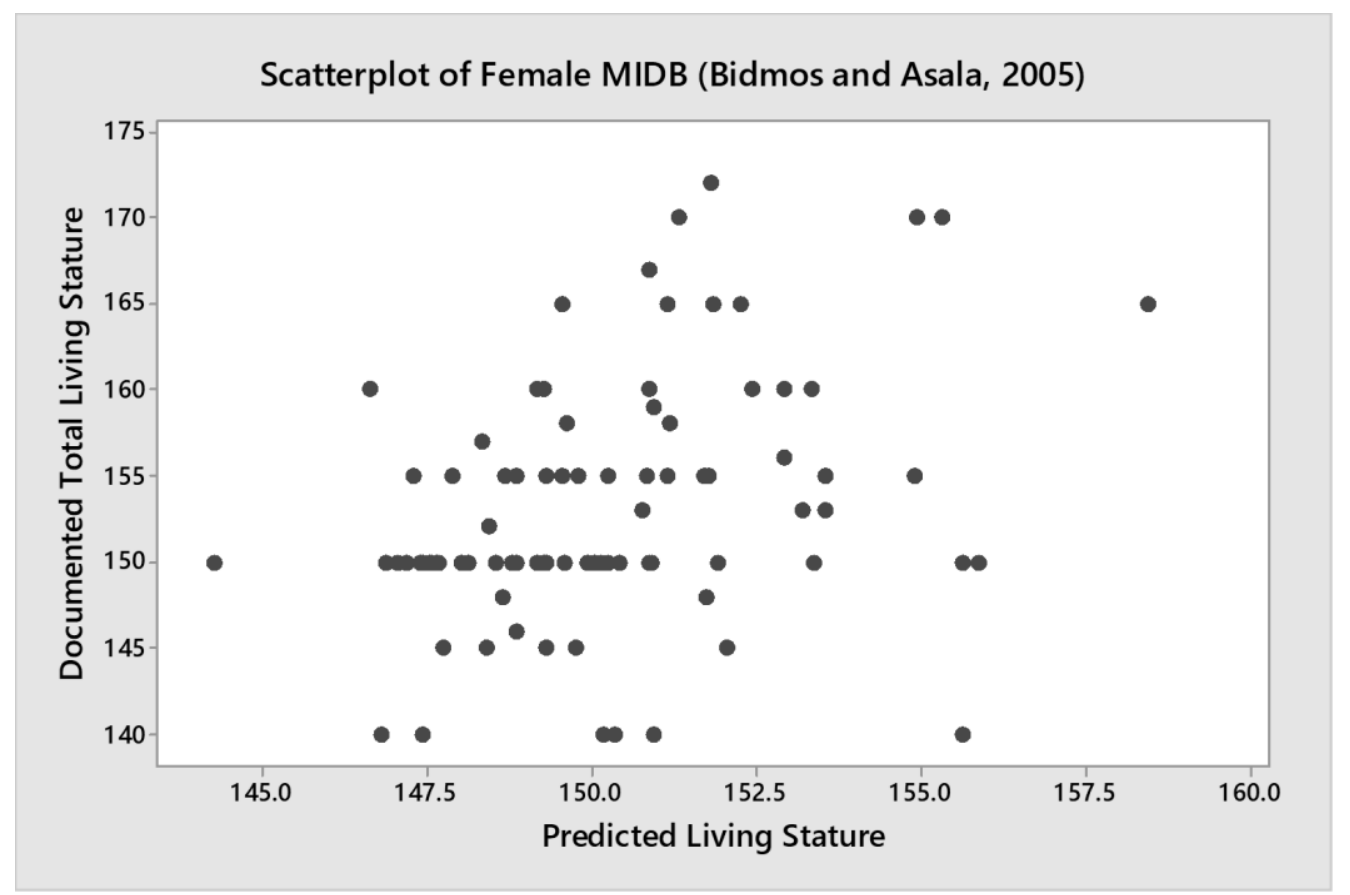

Appendix D12: Simple scatterplot showing the correlation between documented living stature and the predicted living stature, using the female Black South African (Bidmos and Asala, 2005) MIDB simple regression equation. 


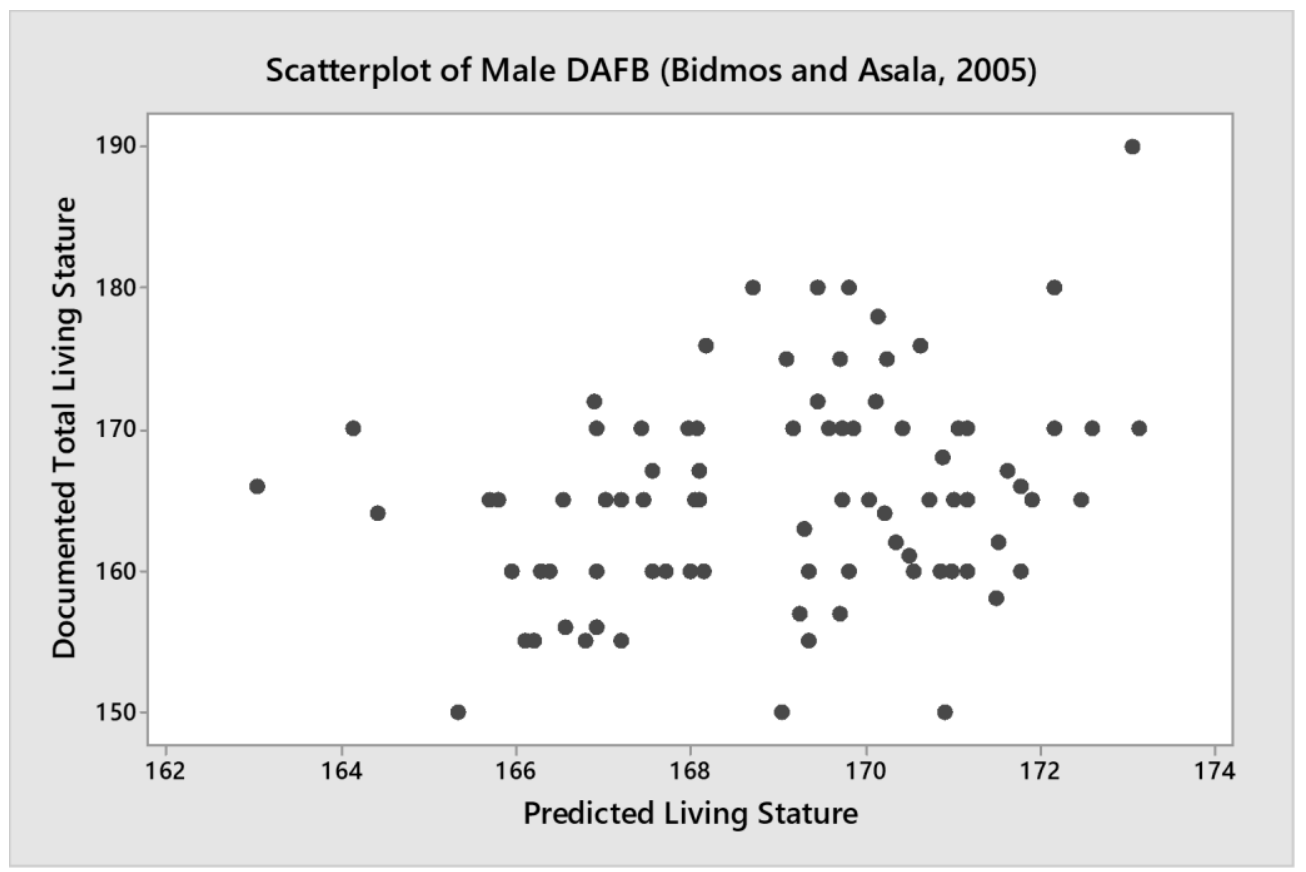

Appendix D13: Simple scatterplot showing the correlation between documented living stature and the predicted living stature, using the male Black South African (Bidmos and Asala, 2005) DAFB simple regression equation.

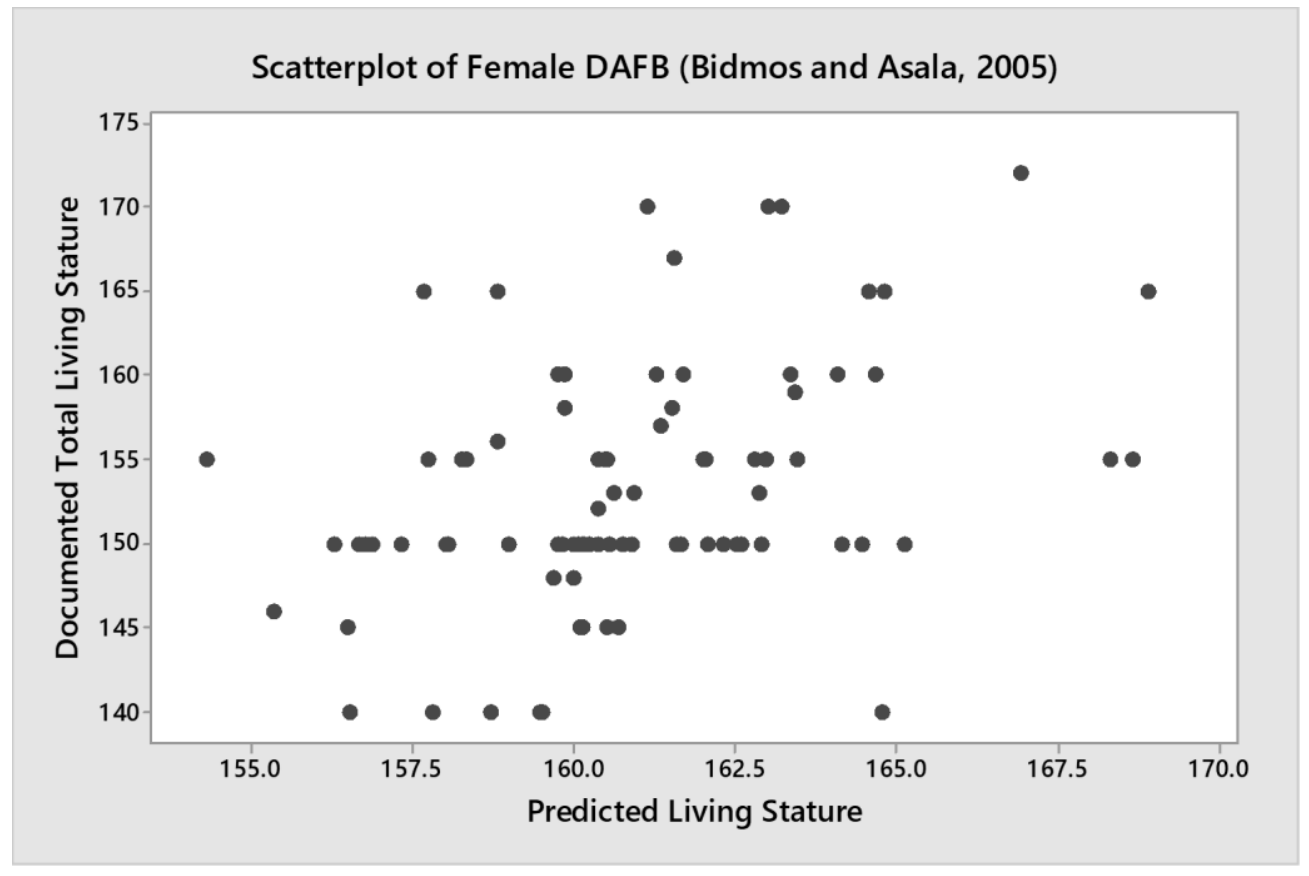

Appendix D14: Simple scatterplot showing the correlation between documented living stature and the predicted living stature, using the female Black South African (Bidmos and Asala, 2005) DAFB simple regression equation. 


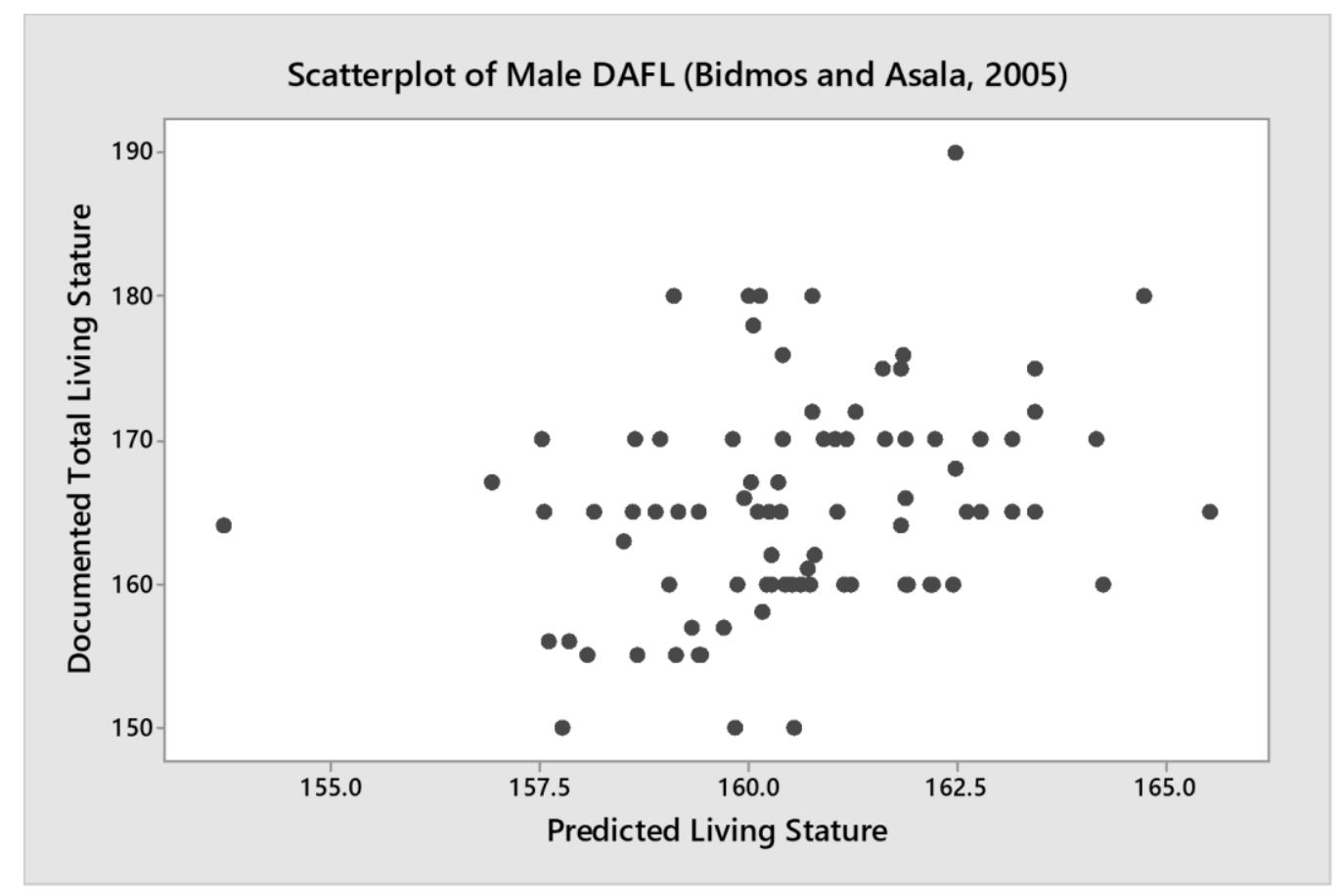

Appendix D15: Simple scatterplot showing the correlation between documented living stature and the predicted living stature, using the male Black South African (Bidmos and Asala, 2005) DAFL simple regression equation.

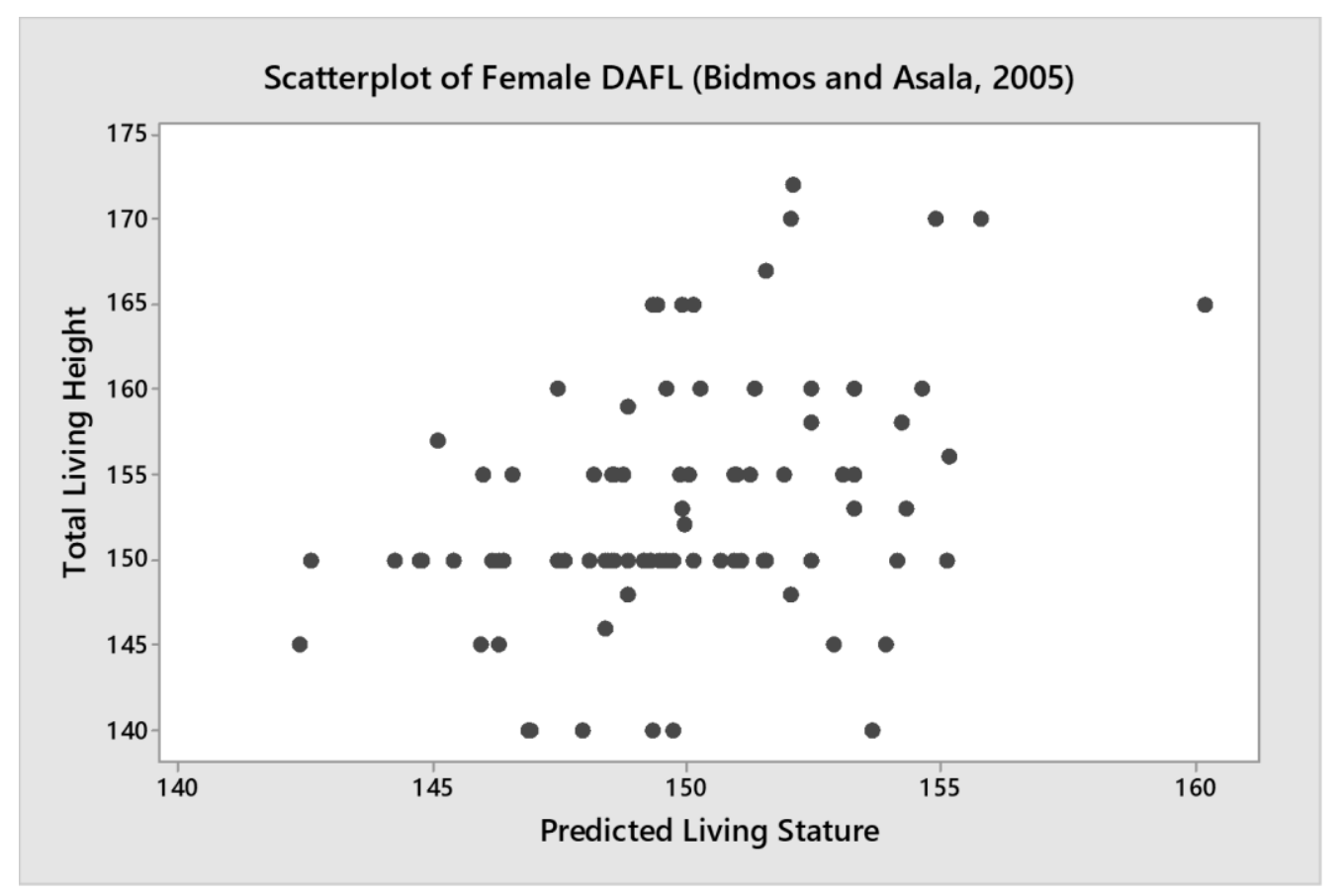

Appendix D16: Simple scatterplot showing the correlation between documented living stature and the predicted living stature, using the female Black South African (Bidmos and Asala, 2005) DAFL simple regression equation. 


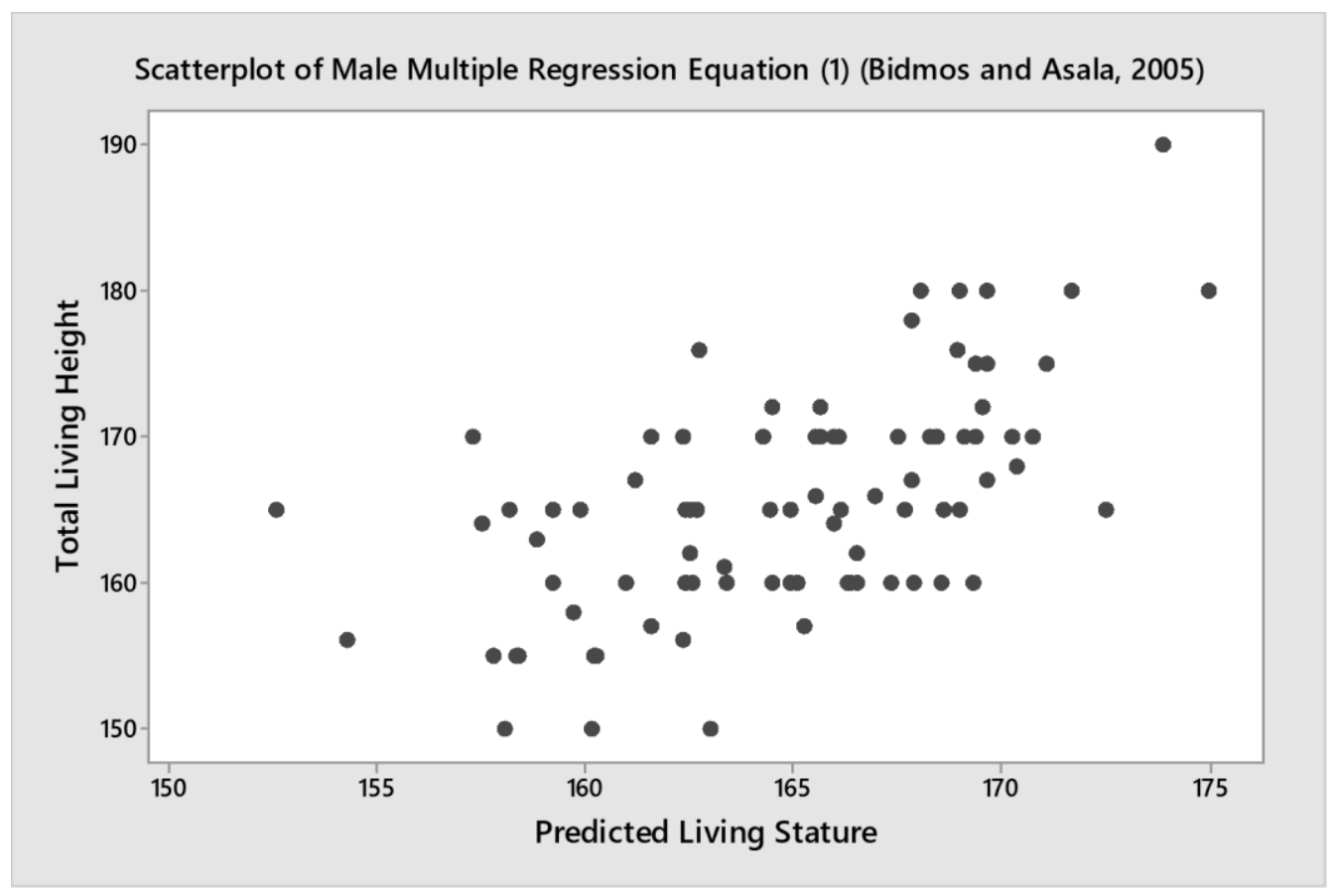

Appendix D17: Simple scatterplot showing the correlation between documented living stature and the predicted living stature, using the male Black South African (Bidmos and Asala, 2005) multiple regression equation 1 (MAXL, MAXH, MIDB, and DAFB).

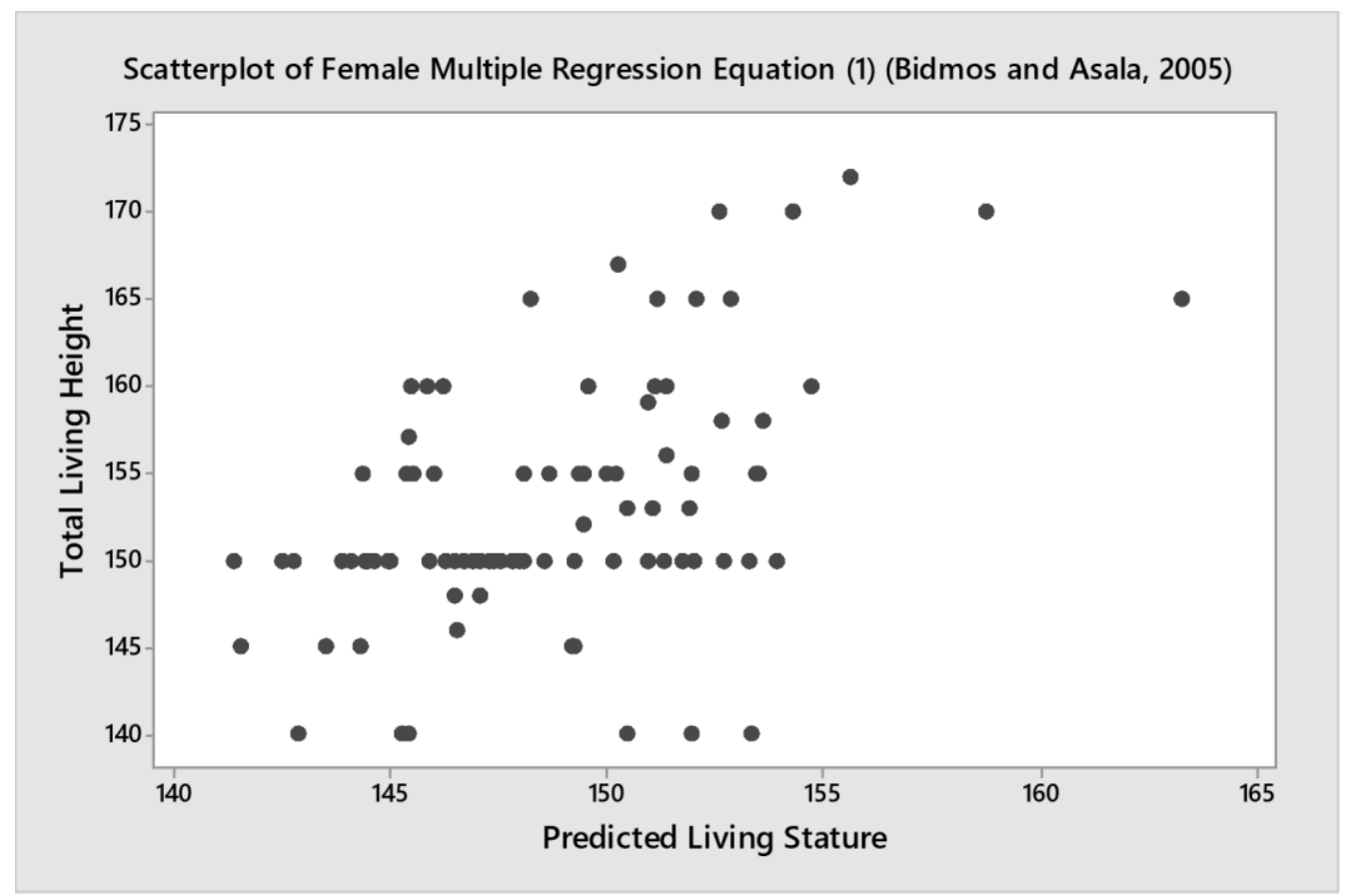

Appendix D18: Simple scatterplot showing the correlation between documented living stature and the predicted living stature, using the female Black South African (Bidmos and Asala, 2005) multiple regression equation 1 (MAXL, LAL, BH, and DAFL). 


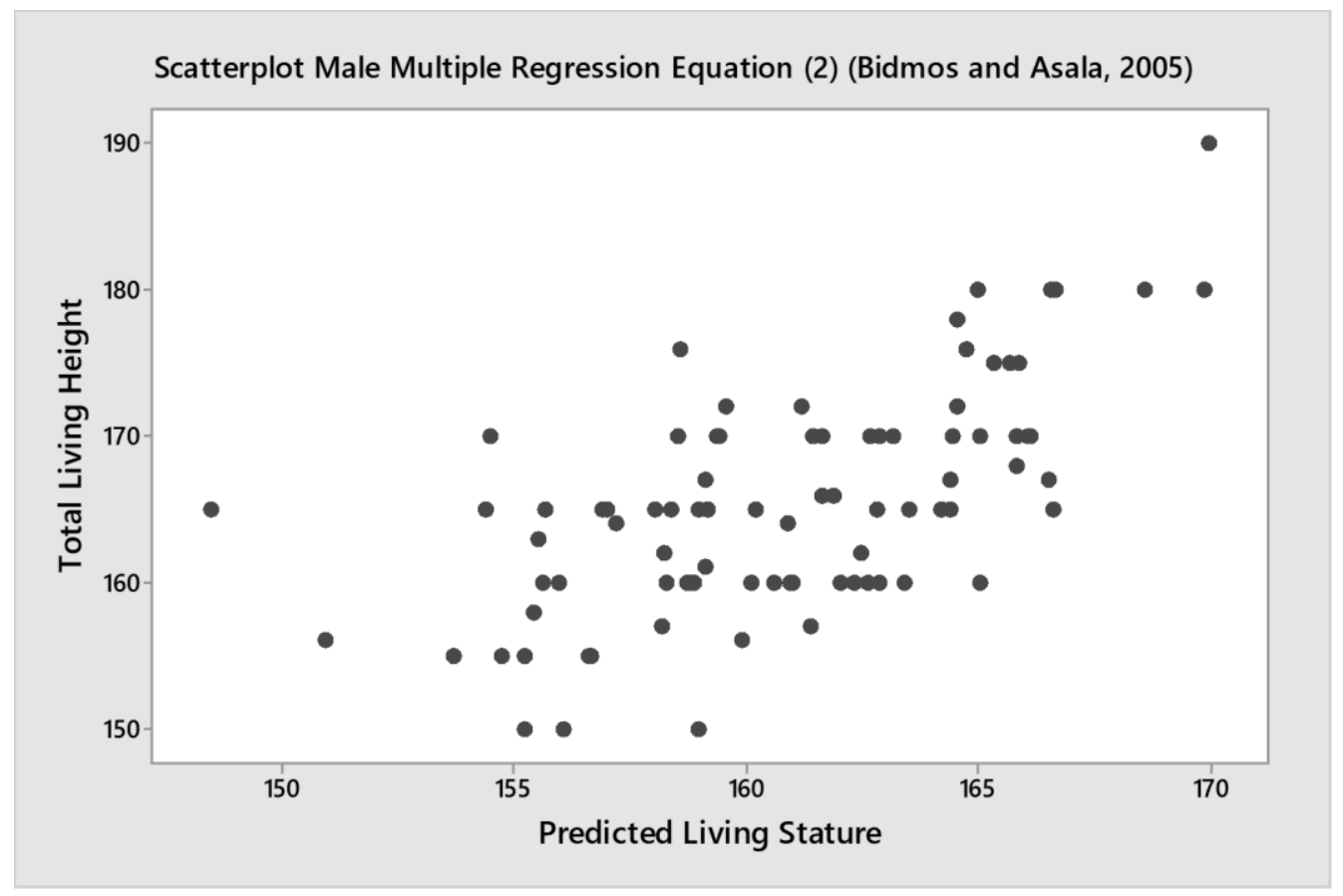

Appendix D19: Simple scatterplot showing the correlation between documented living stature and the predicted living stature, using the male Black South African (Bidmos and Asala, 2005) multiple regression equation 2 (MAXL, MAXH, and MIDB).

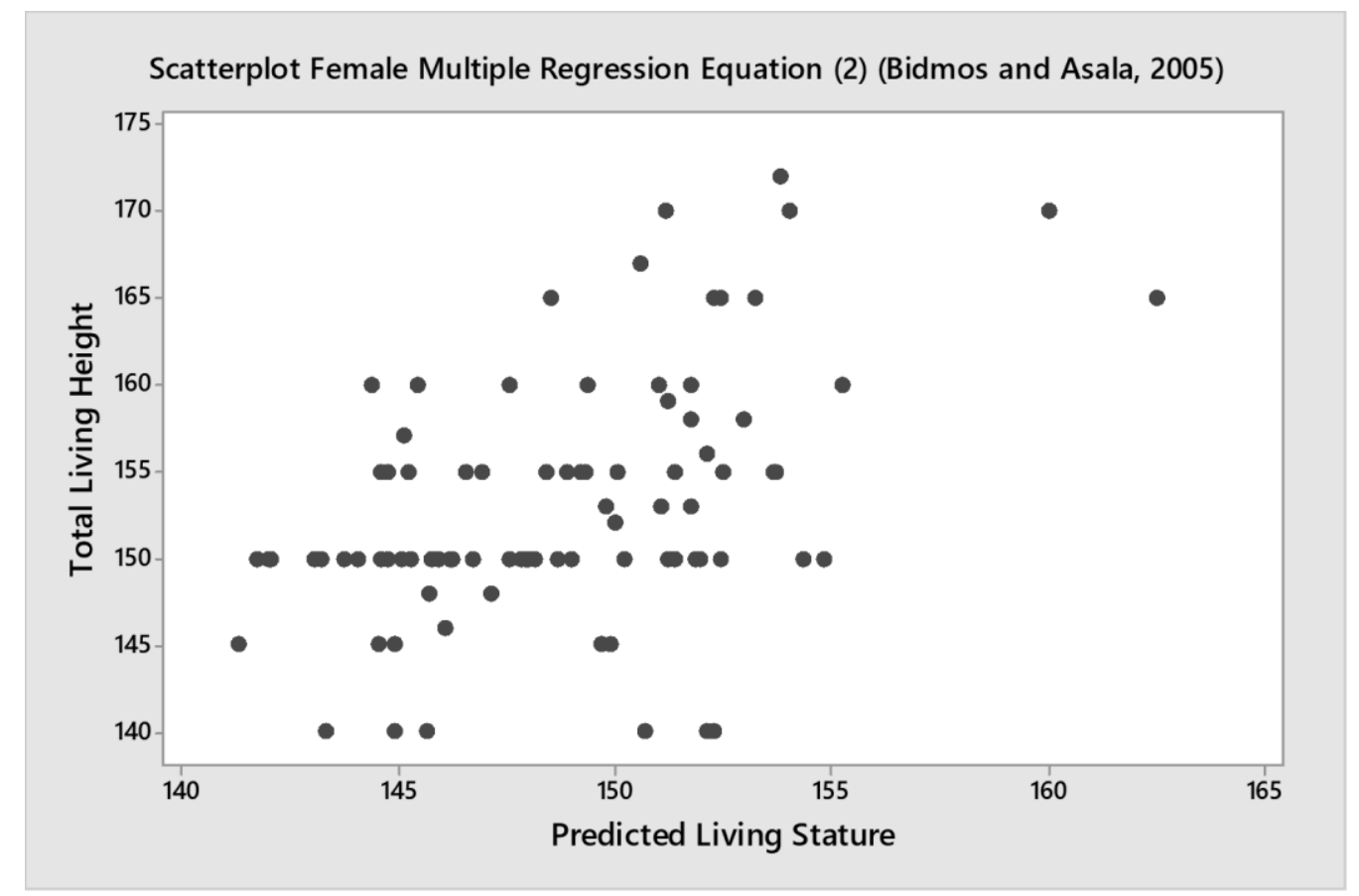

Appendix D20: Simple scatterplot showing the correlation between documented living stature and the predicted living stature, using the female Black South African (Bidmos and Asala, 2005) multiple regression equation 2 (MAXL, LAL, and DAFL). 


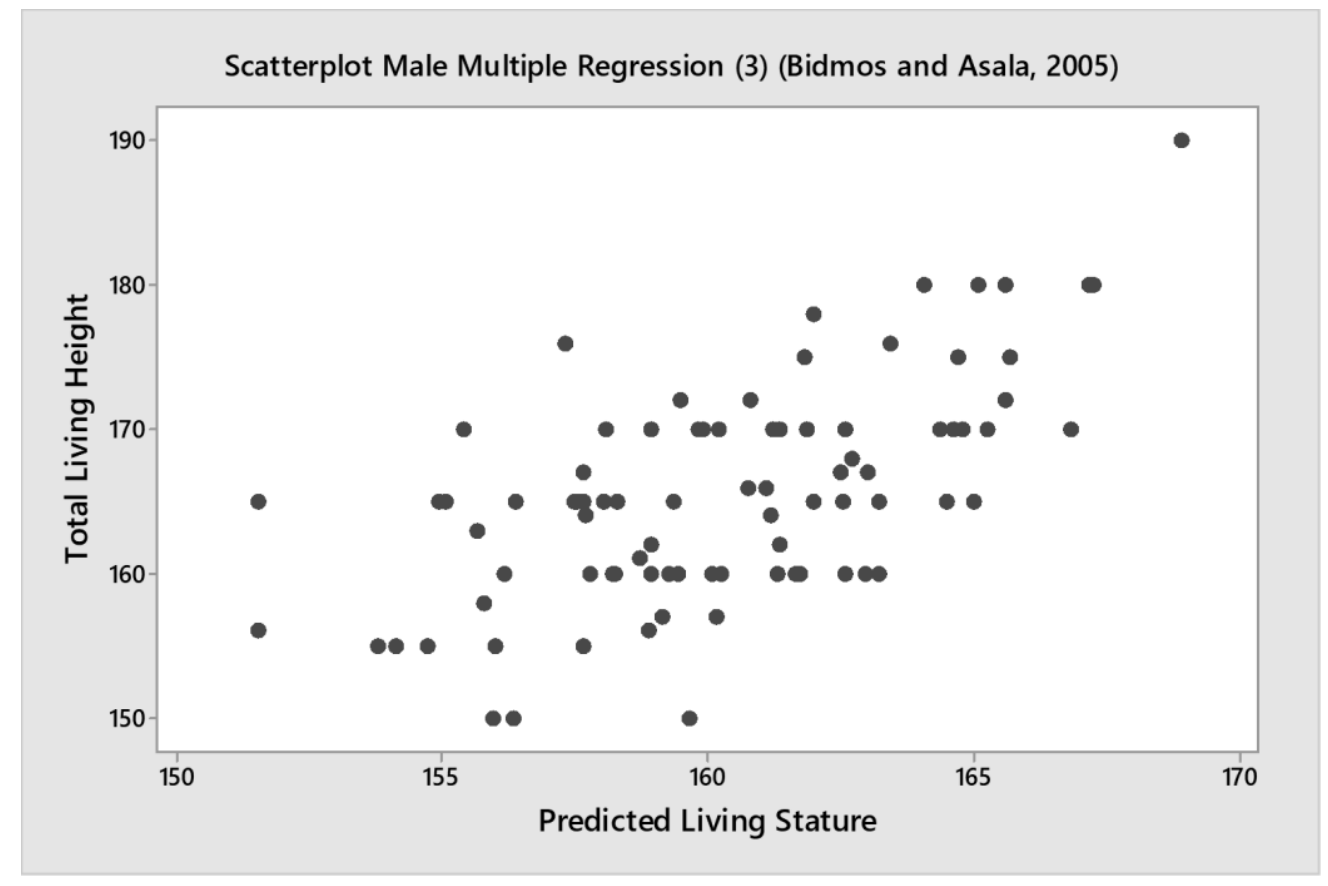

Appendix D21: Simple scatterplot showing the correlation between documented living stature and the predicted living stature, using the male Black South African (Bidmos and Asala, 2005) multiple regression equation 3 (MAXL and MIDB).

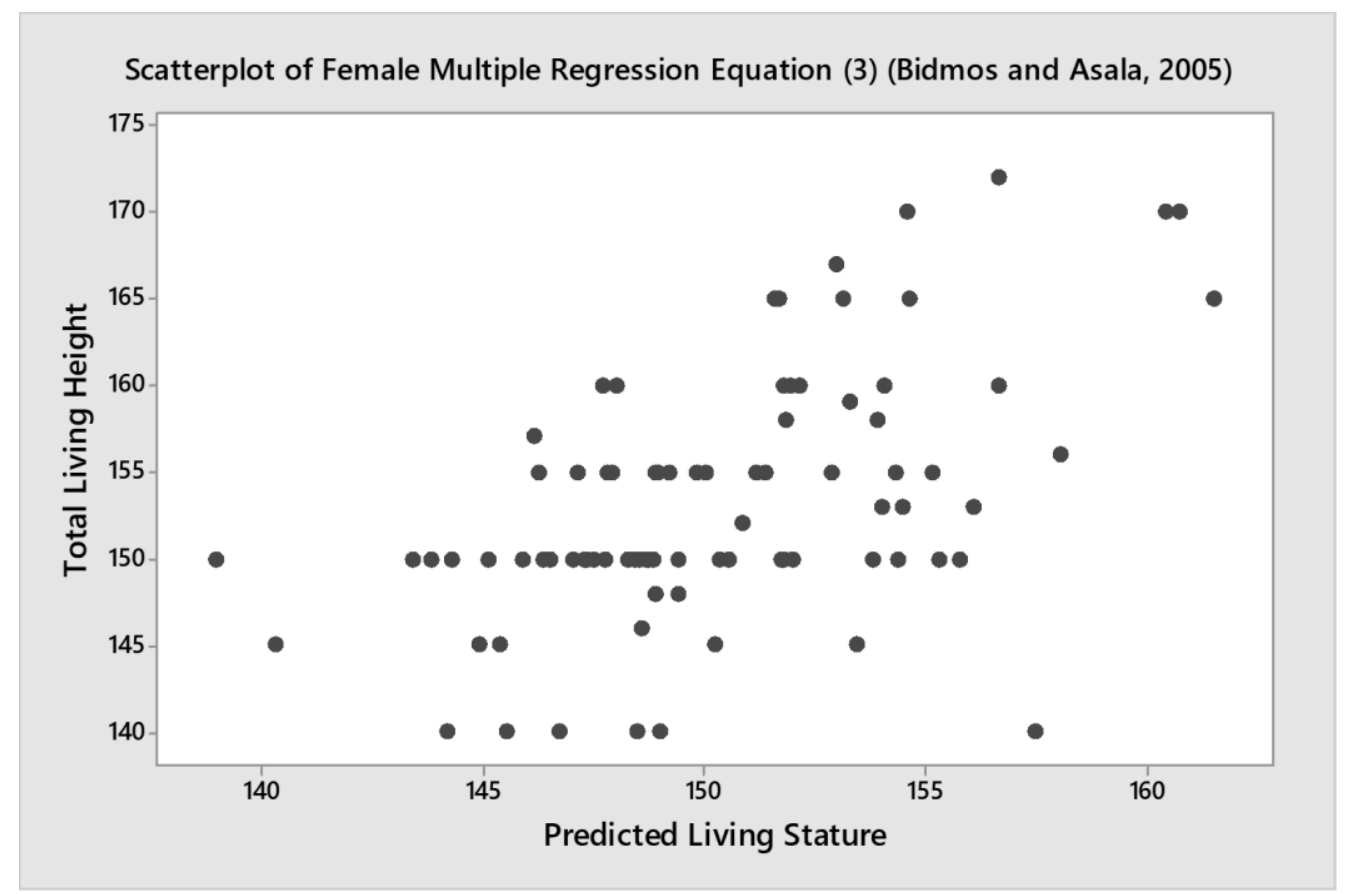

Appendix D22: Simple scatterplot showing the correlation between documented living stature and the predicted living stature, using the female Black South African (Bidmos and Asala, 2005) multiple regression equation 3 (MAXL, MAXH, and DAFL). 


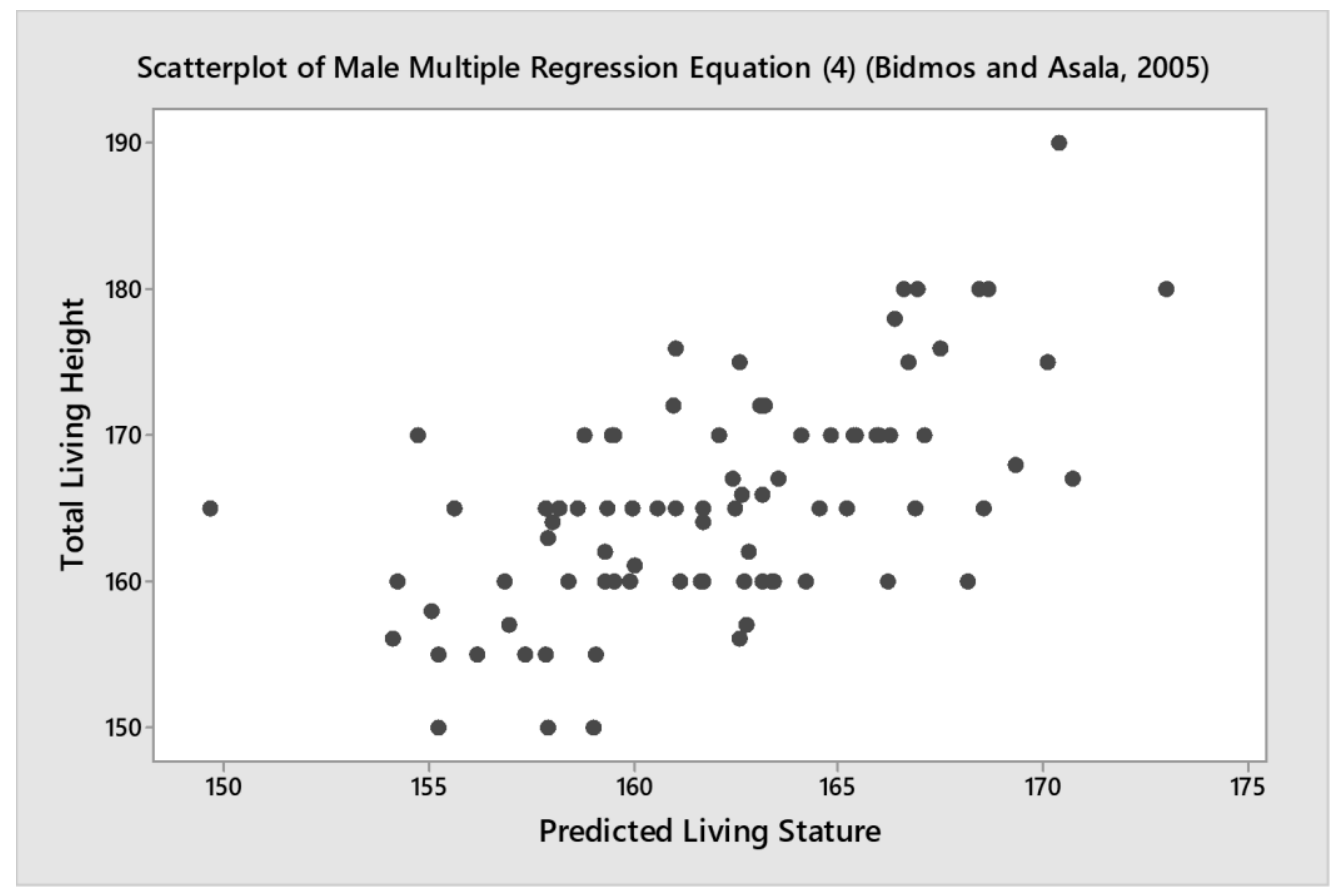

Appendix D23: Simple scatterplot showing the correlation between documented living stature and the predicted living stature, using the male Black South African (Bidmos and Asala, 2005) multiple regression equation 4 (MAXL and MAXH).

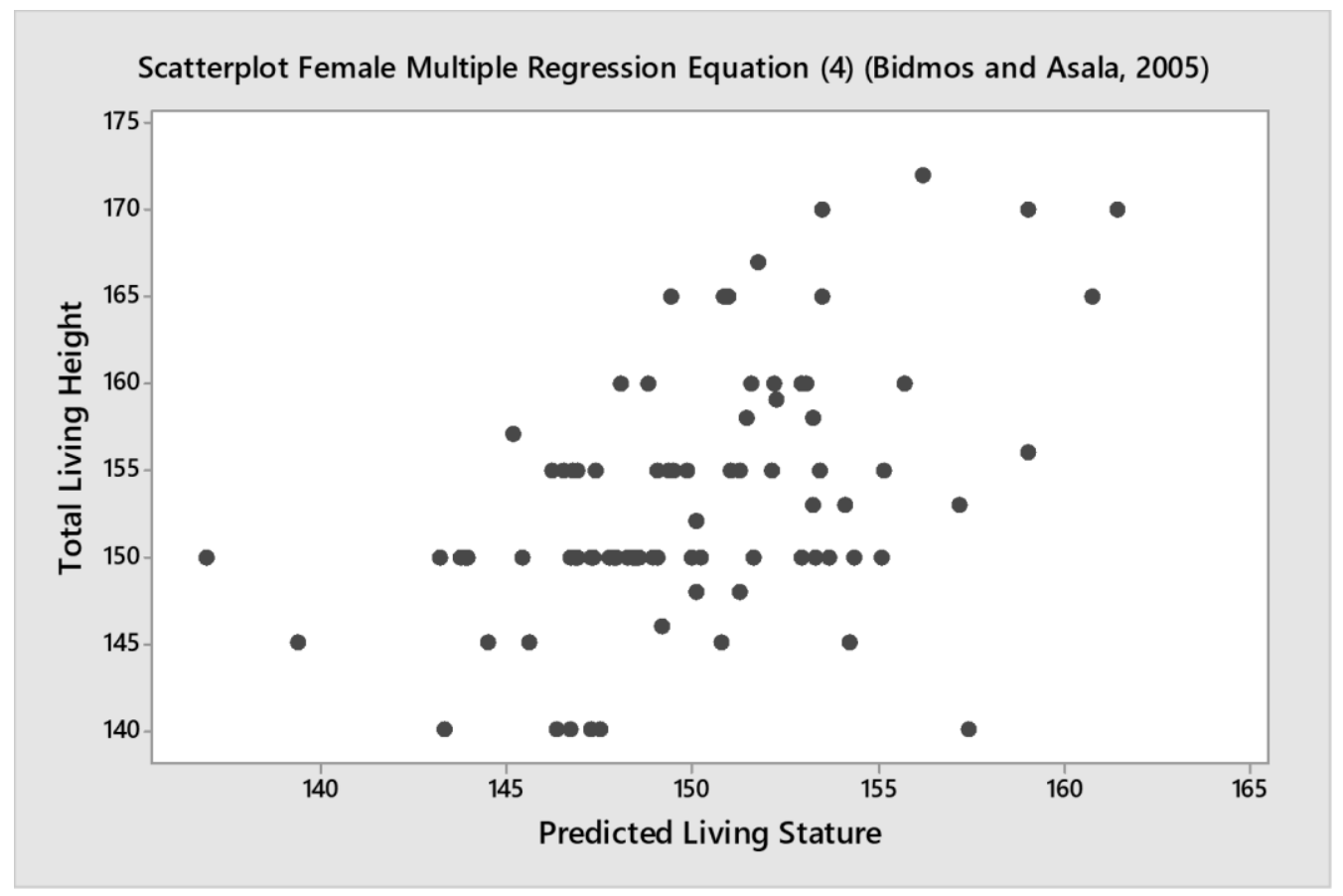

Appendix D24: Simple scatterplot showing the correlation between documented living stature and the predicted living stature, using the female Black South African (Bidmos and Asala, 2005) multiple regression equation 4 (MAXH and DAFL). 


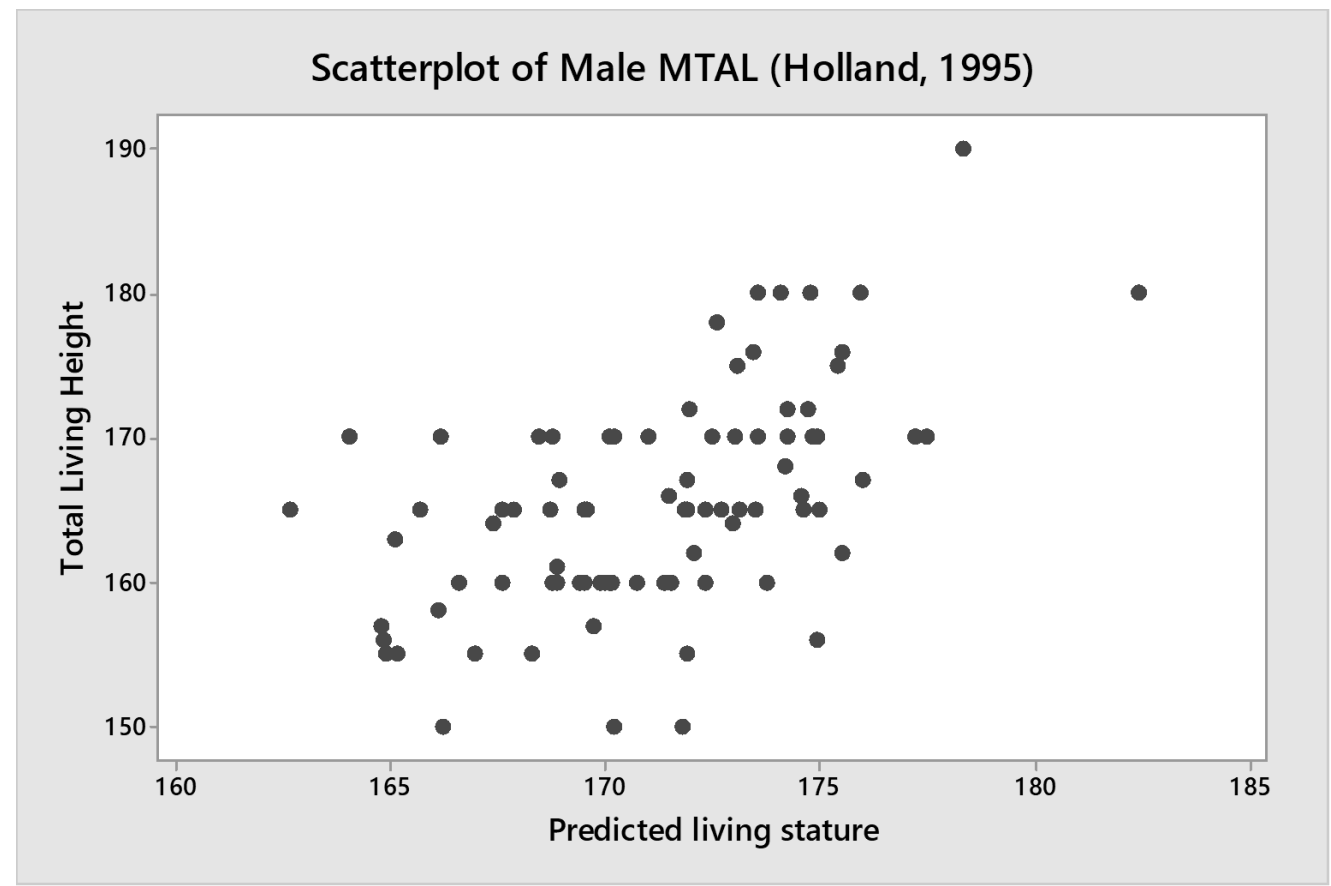

Appendix D25: Simple scatterplot showing the correlation between documented living stature and the predicted living stature, using the male Black American (Holland, 1995) MTAL simple regression equation.

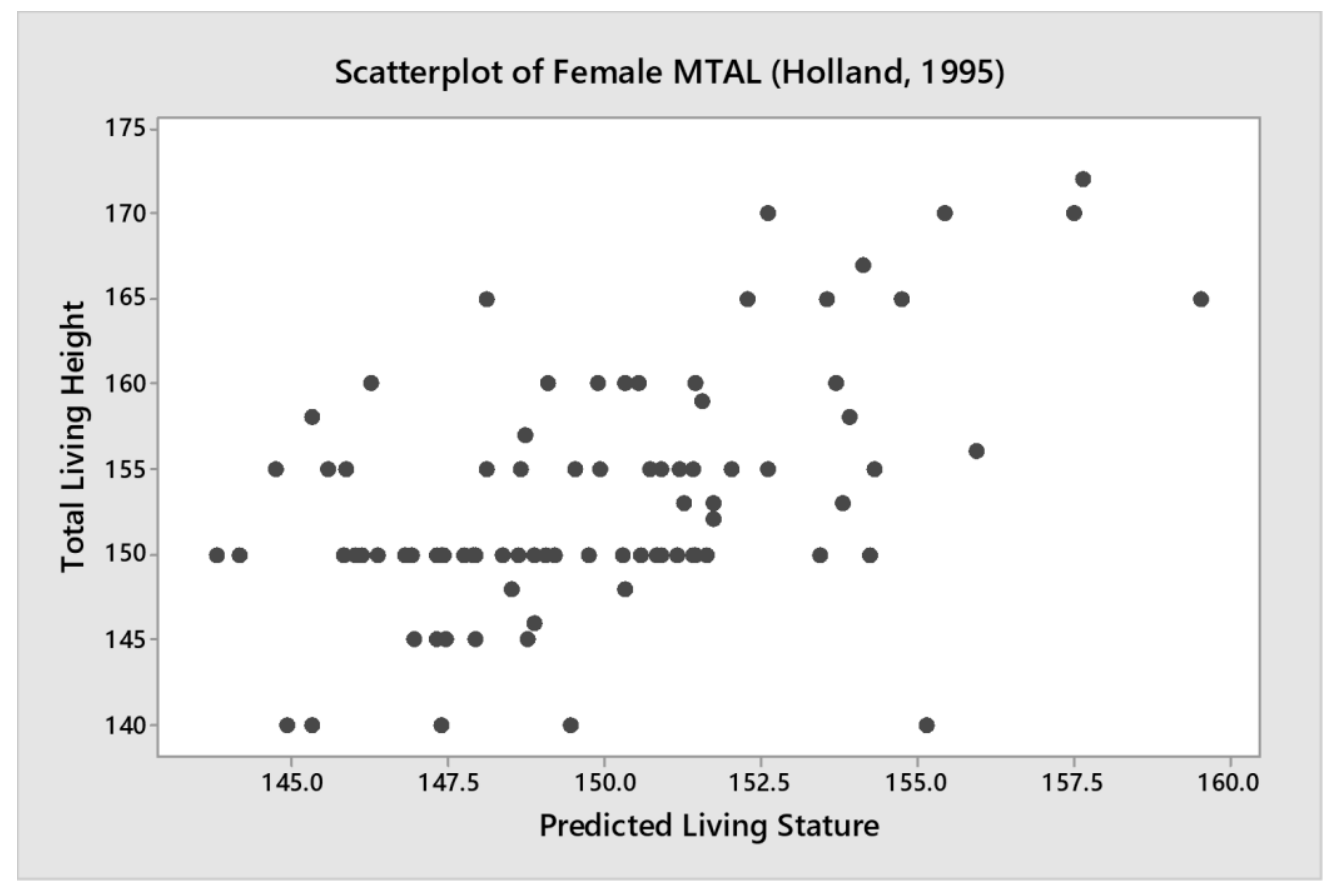

Appendix D26: Simple scatterplot showing the correlation between documented living stature and the predicted living stature, using the female Black American (Holland, 1995) MTAL simple regression equation. 
APPENDIX E:

ACCURACIES OF CROSS-VALIDATION 


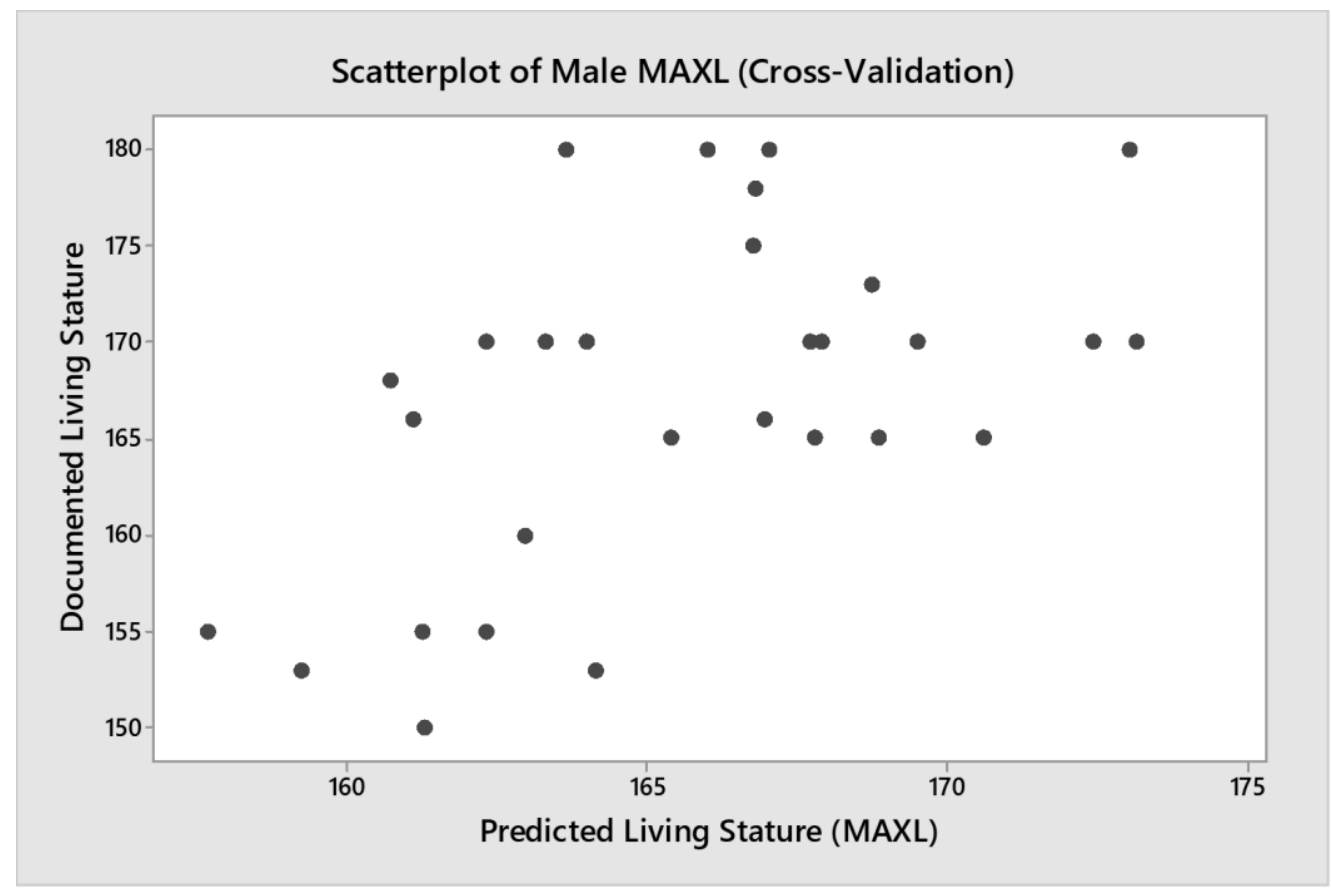

Appendix E1: Simple scatterplot illustrating the correlation between documented and predicted living stature, in a Thai population, using the male MAXL calcaneal simple regression equation.

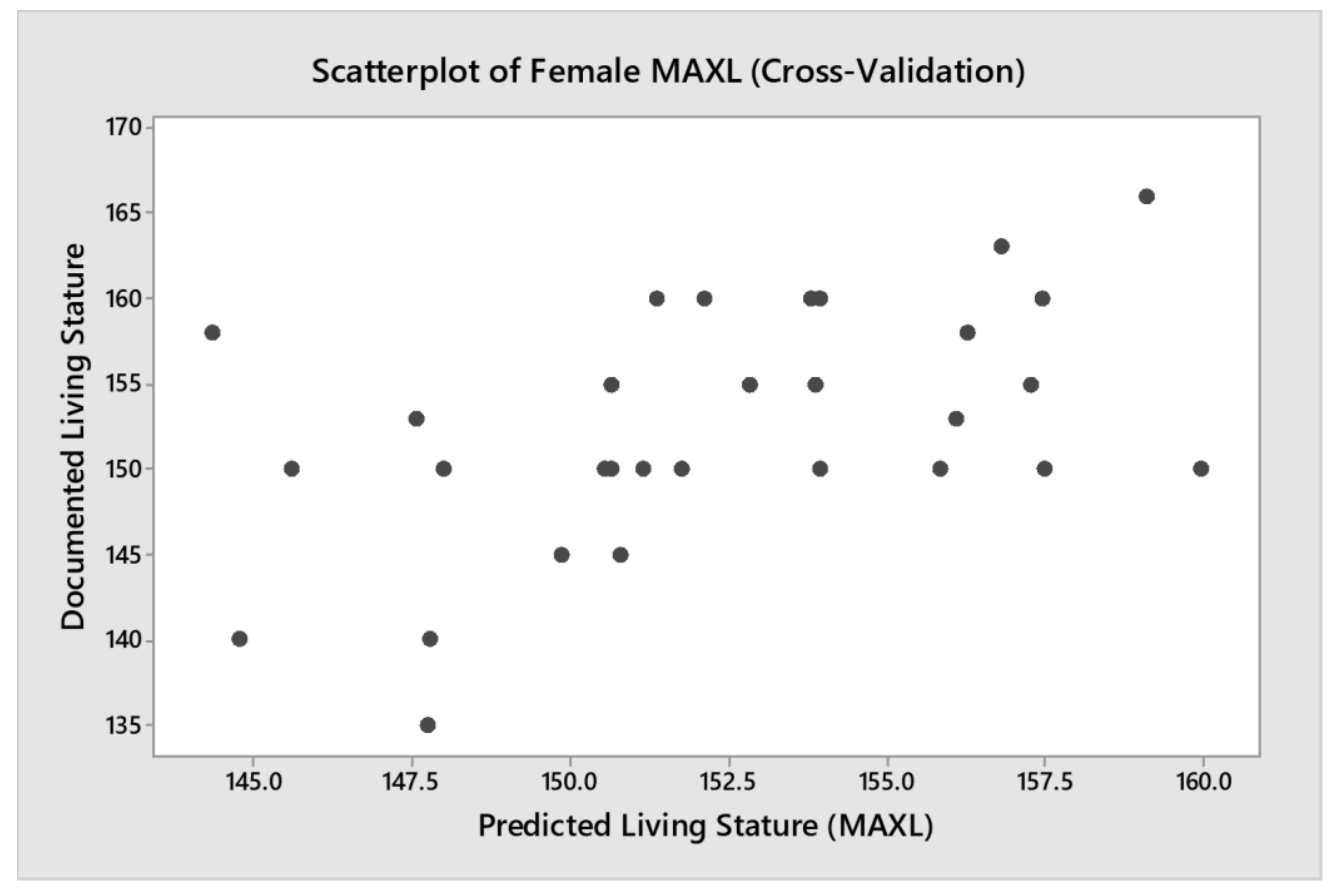

Appendix E2: Simple scatterplot illustrating the correlation between documented and predicted living stature, in a Thai population, using the female MAXL calcaneal simple regression equation. 


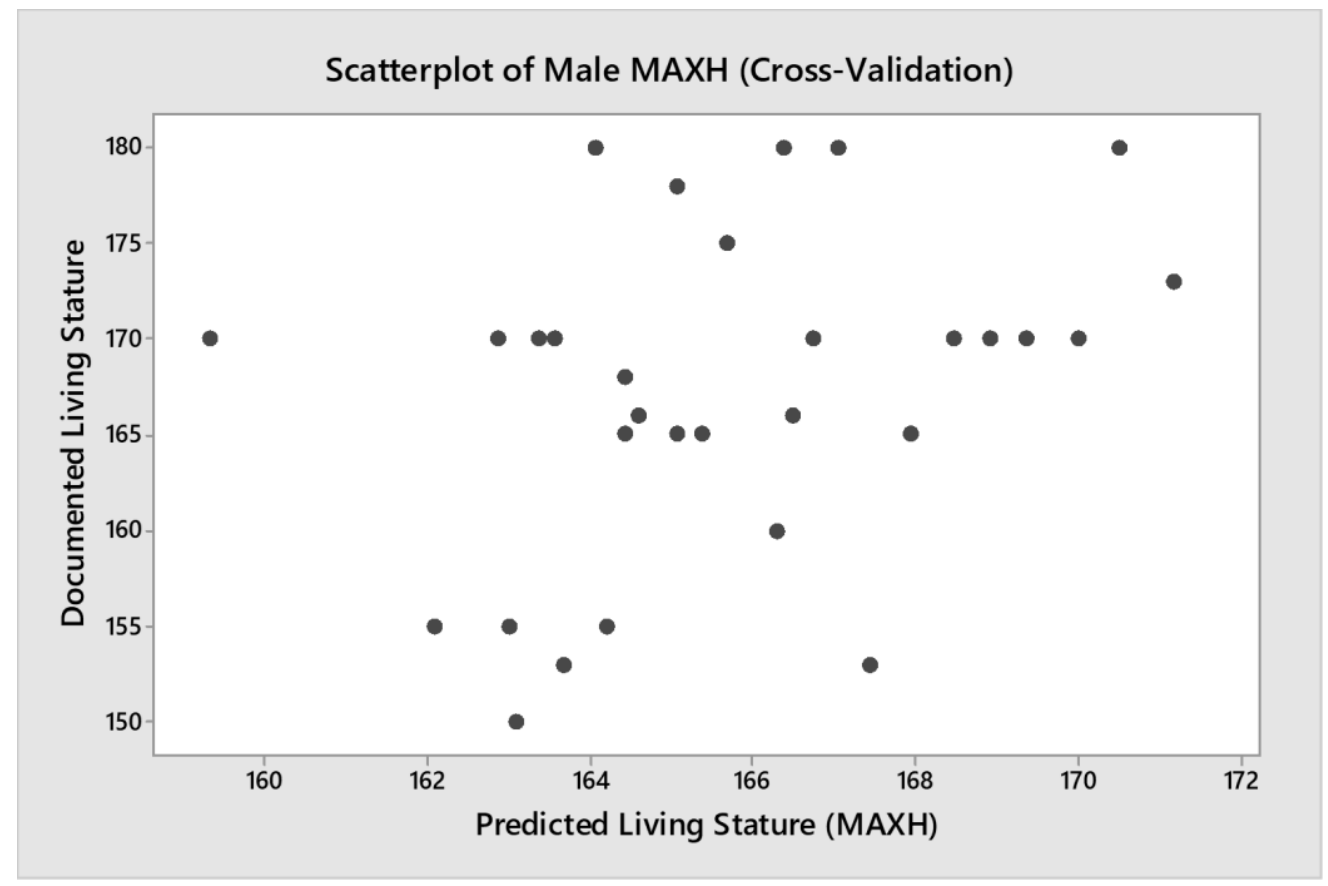

Appendix E3: Simple scatterplot illustrating the correlation between documented and predicted living stature, in a Thai population, using the male MAXH calcaneal simple regression equation.

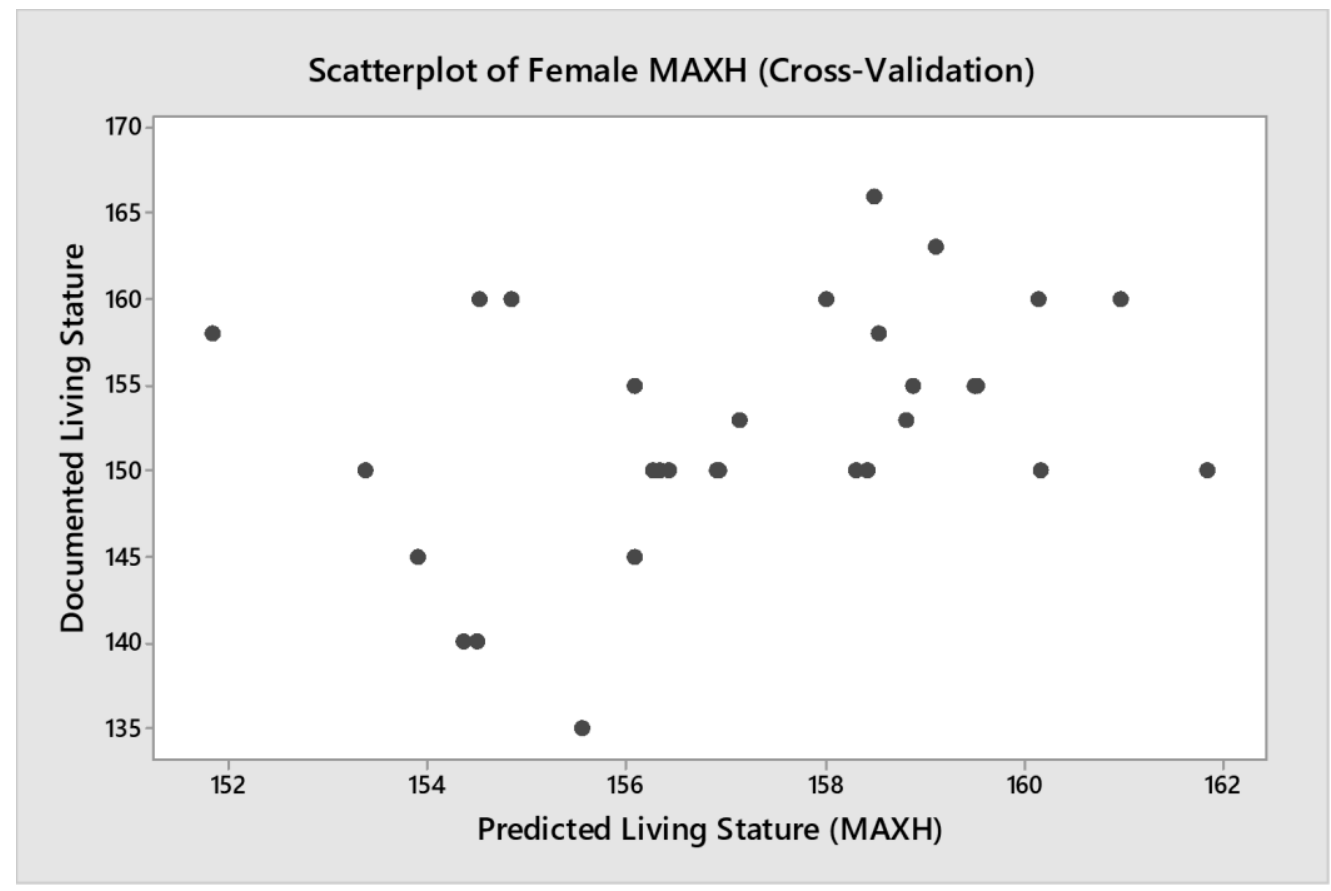

Appendix E4: Simple scatterplot illustrating the correlation between documented and predicted living stature, in a Thai population, using the female MAXH calcaneal simple regression equation. 


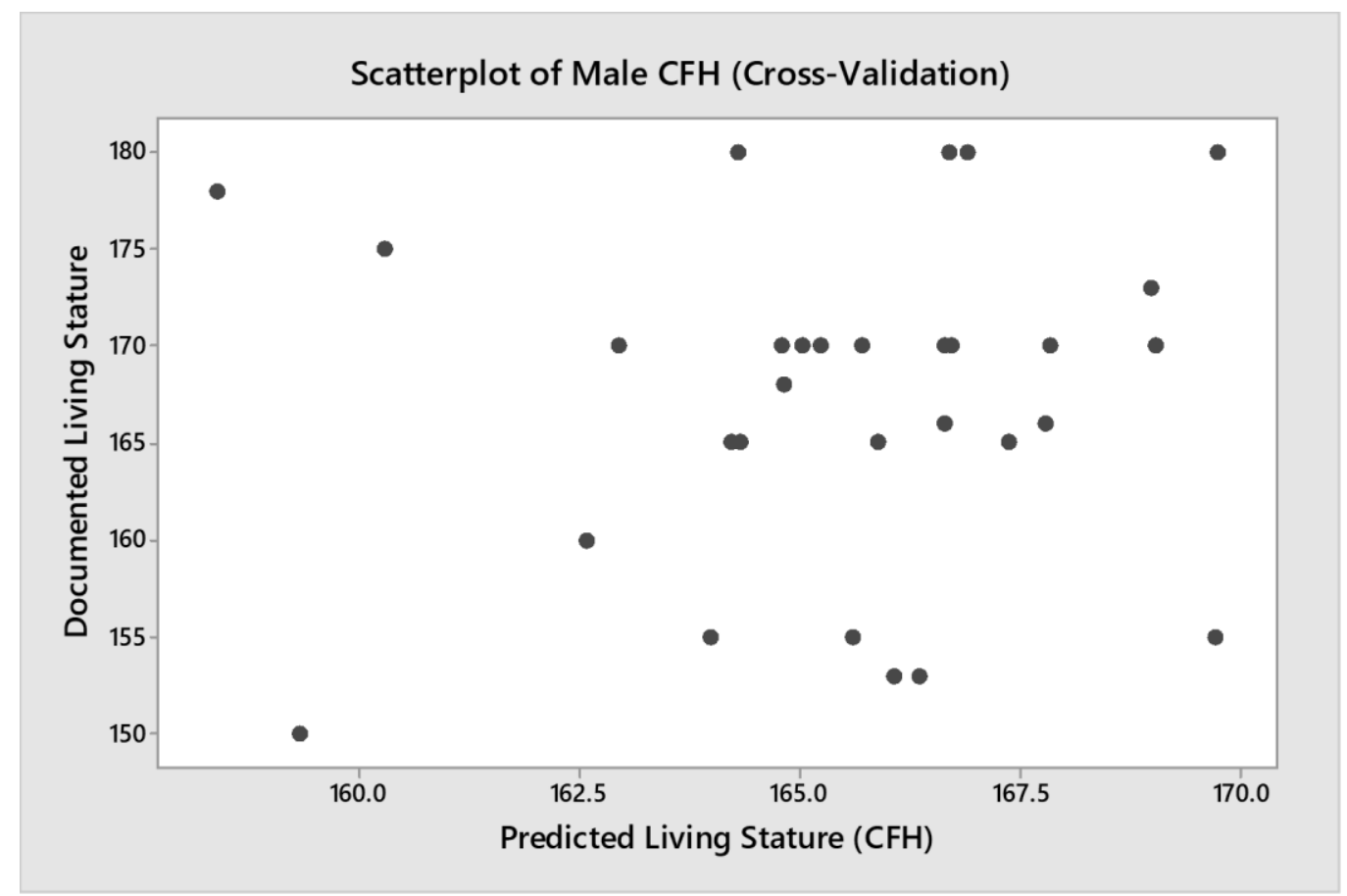

Appendix E5: Simple scatterplot illustrating the correlation between documented and predicted living stature, in a Thai population, using the male $\mathrm{CFH}$ calcaneal simple regression equation. 


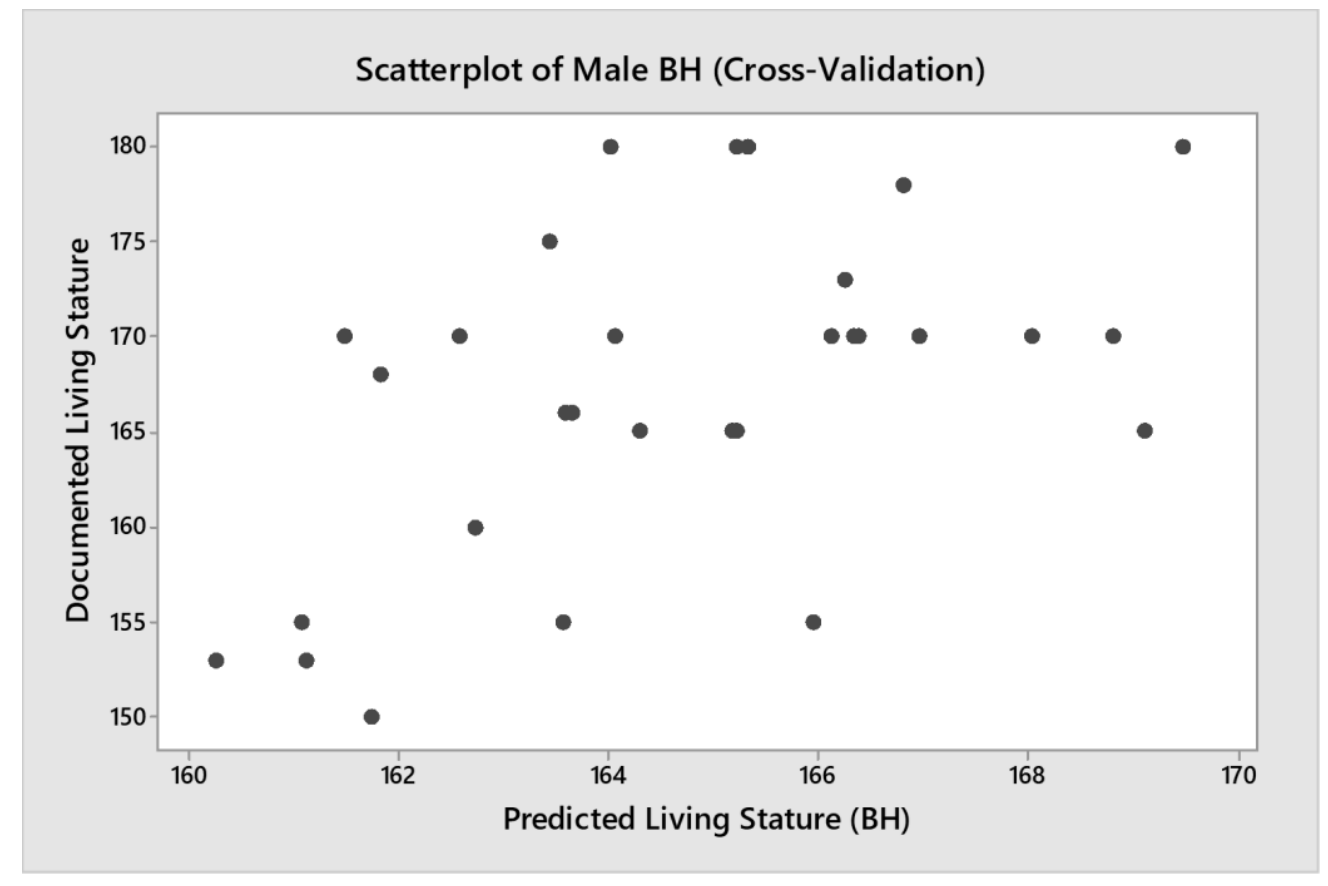

Appendix E6: Simple scatterplot illustrating the correlation between documented and predicted living stature, in a Thai population, using the male $\mathrm{BH}$ calcaneal simple regression equation.

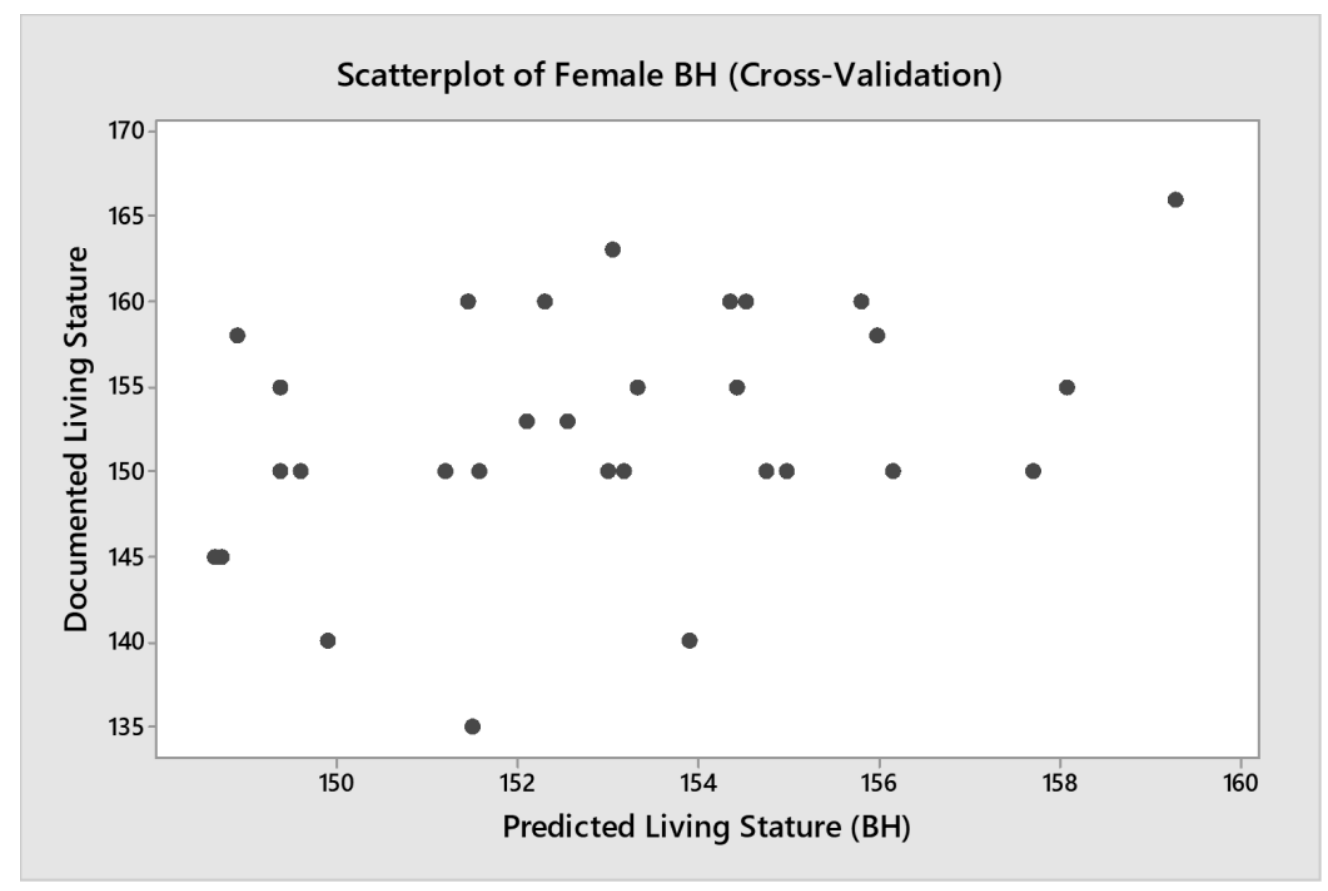

Appendix E7: Simple scatterplot illustrating the correlation between documented and predicted living stature, in a Thai population, using the female $\mathrm{BH}$ calcaneal simple regression equation. 


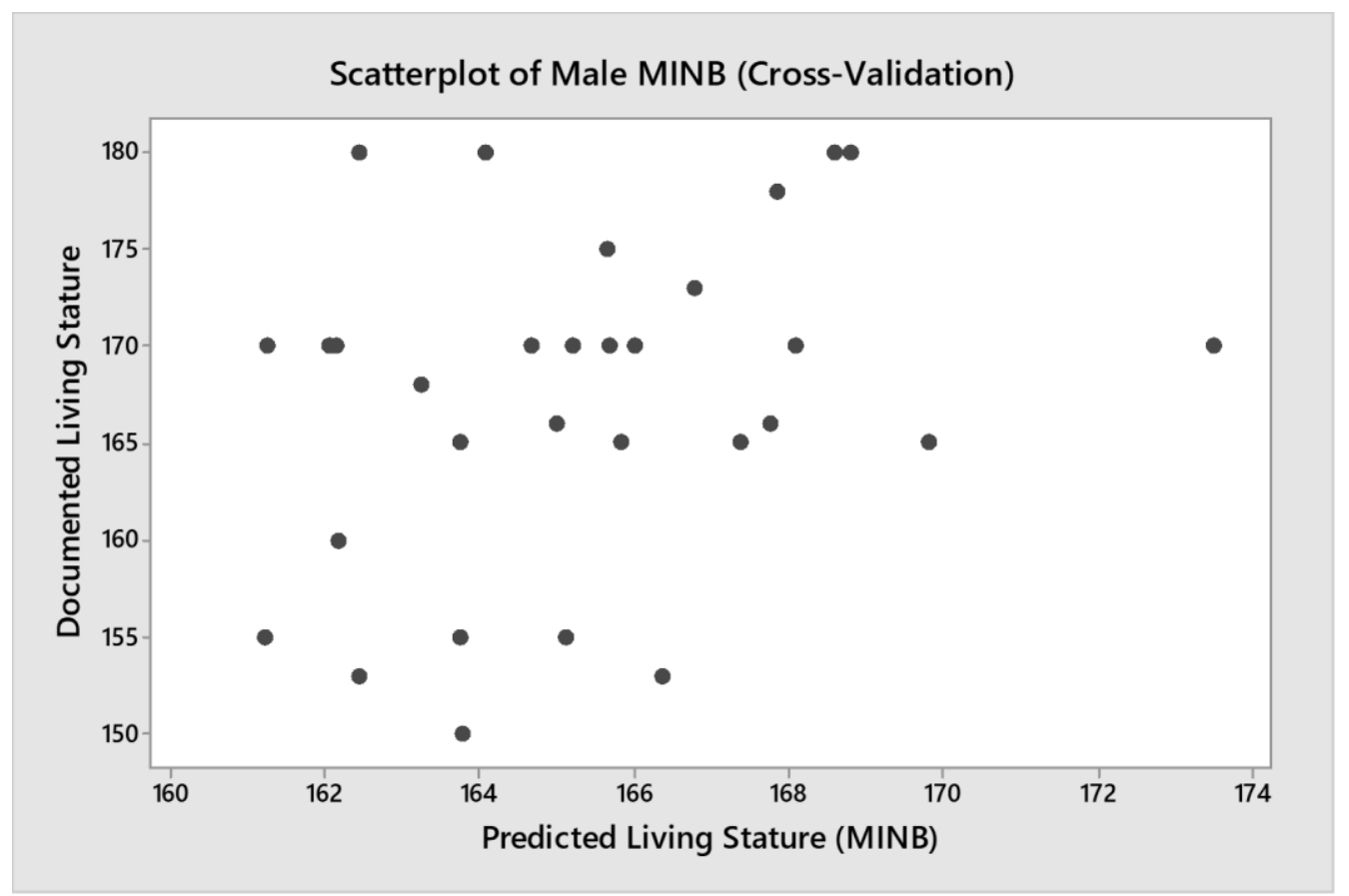

Appendix E8: Simple scatterplot illustrating the correlation between documented and predicted living stature, in a Thai population, using the male MINB calcaneal simple regression equation. 


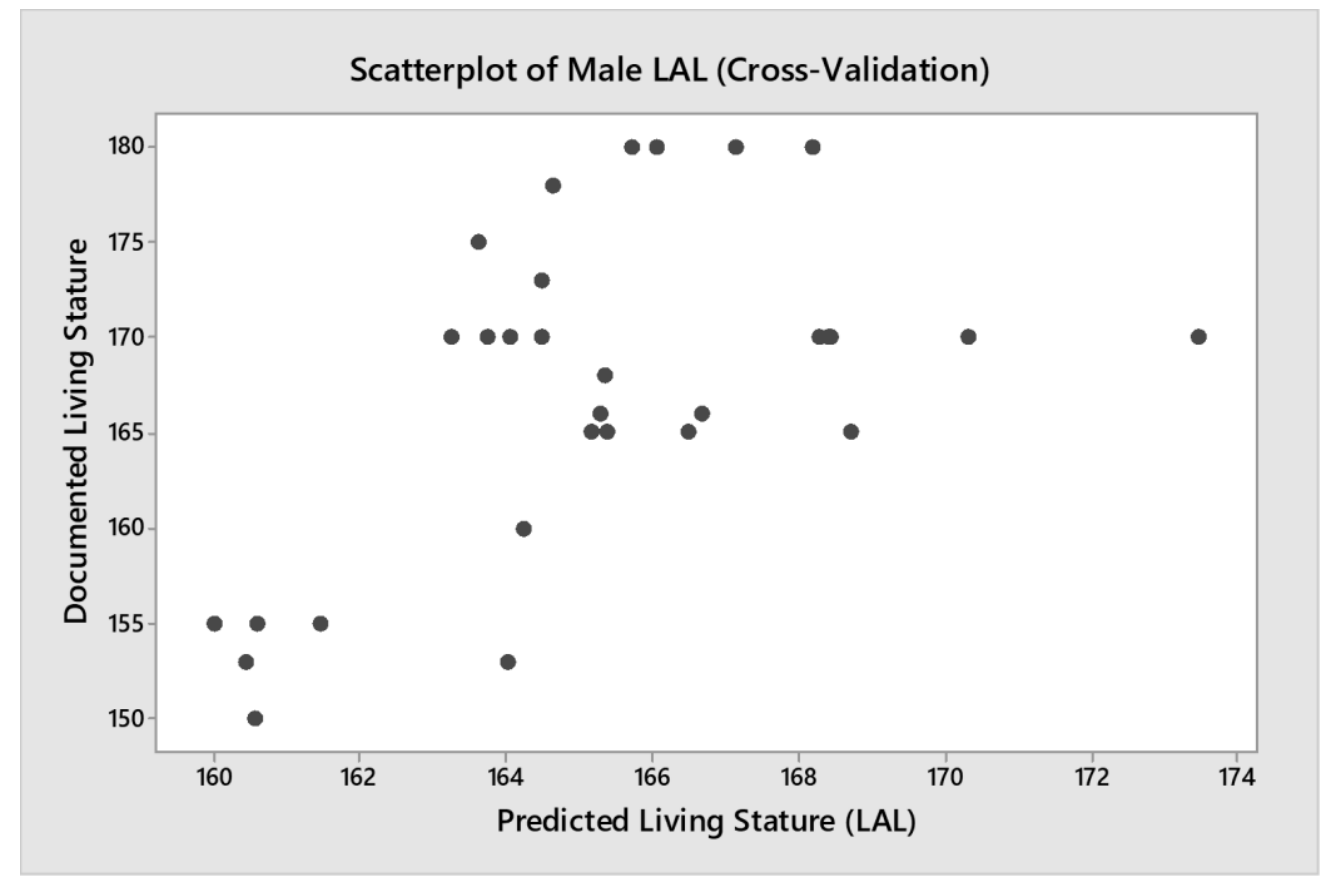

Appendix E9: Simple scatterplot illustrating the correlation between documented and predicted living stature, in a Thai population, using the male LAL calcaneal simple regression equation.

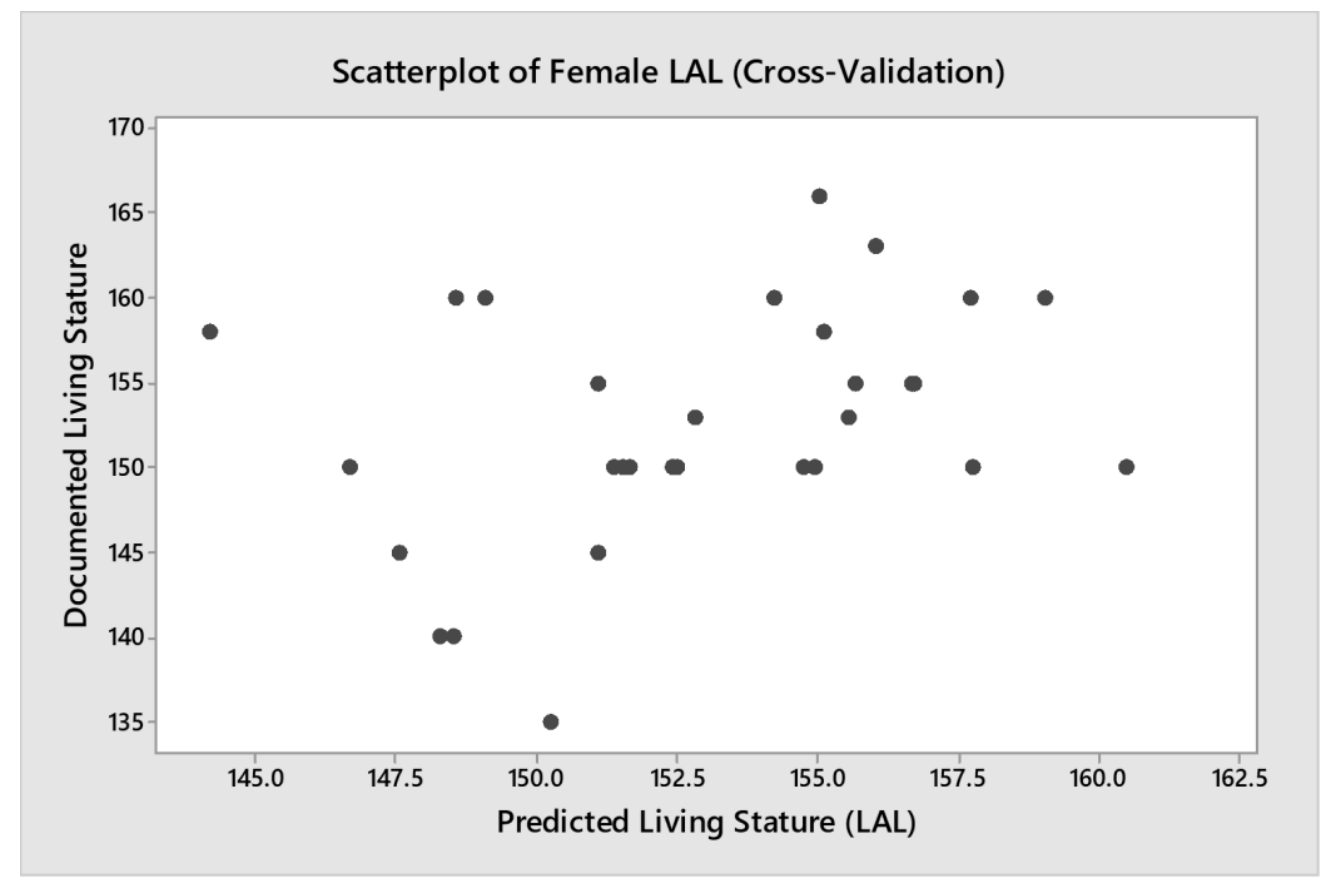

Appendix E10: Simple scatterplot illustrating the correlation between documented and predicted living stature, in a Thai population, using the female LAL calcaneal simple regression equation. 


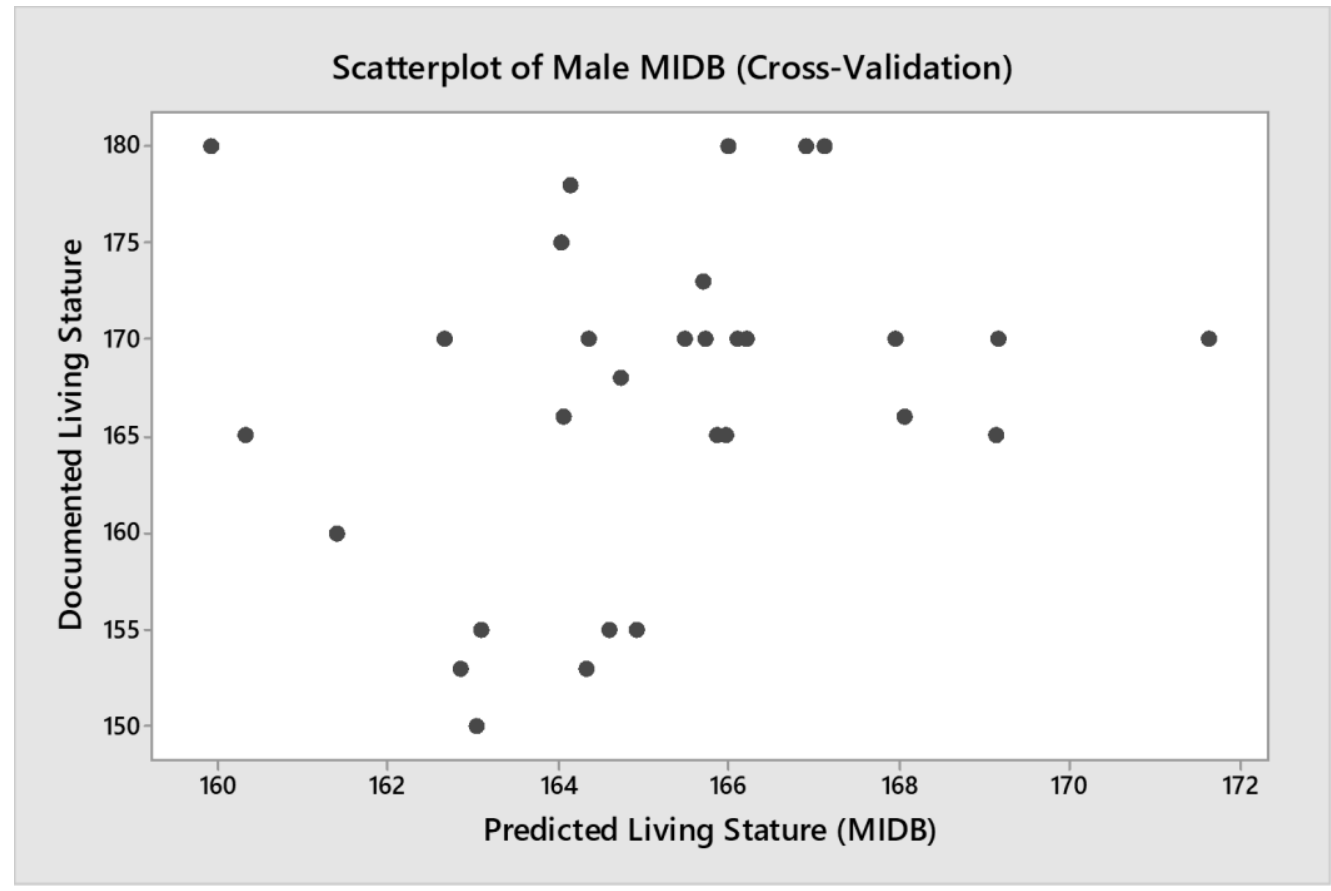

Appendix E11: Simple scatterplot illustrating the correlation between documented and predicted living stature, in a Thai population, using the male MIDB calcaneal simple regression equation.

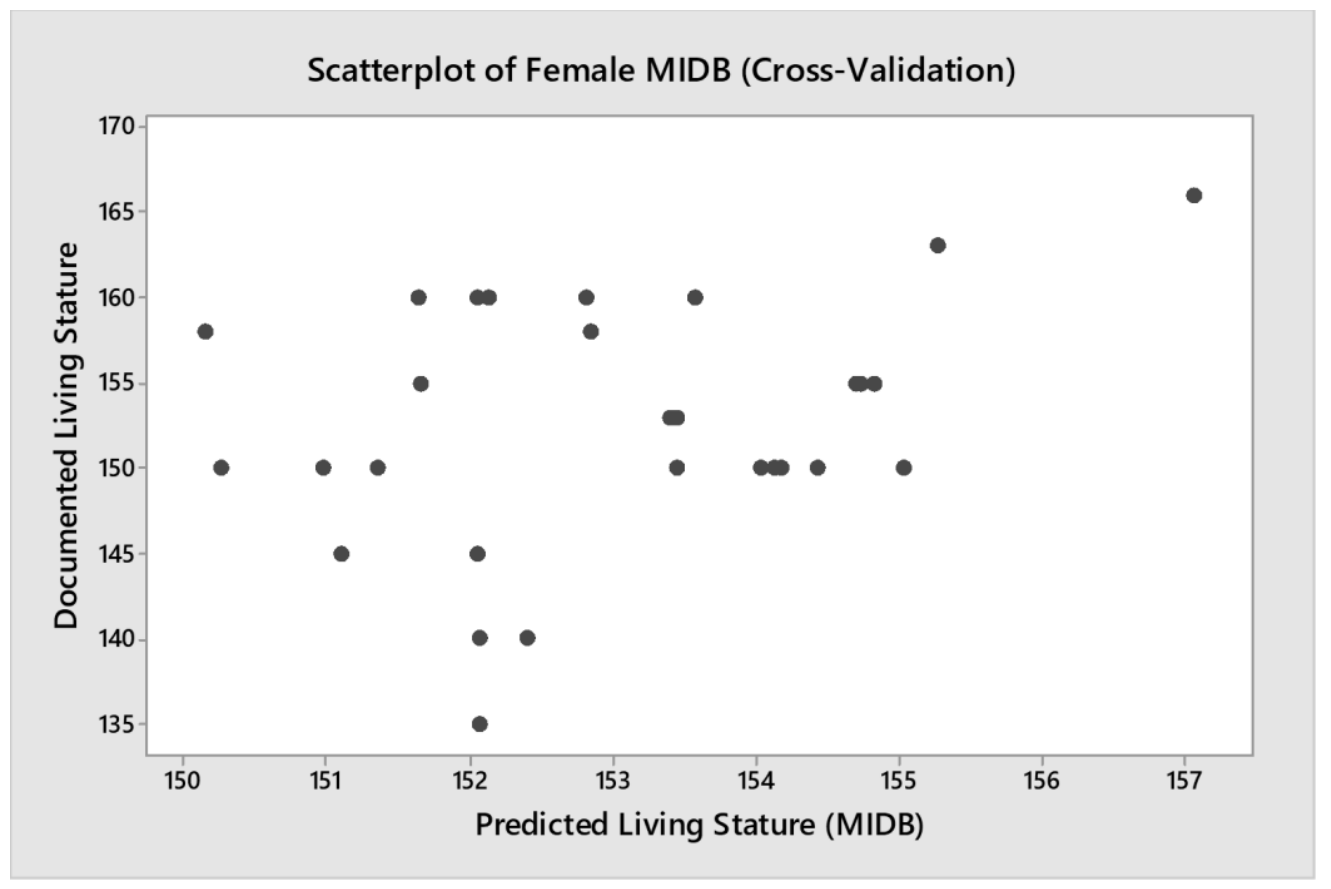

Appendix E12: Simple scatterplot illustrating the correlation between documented and predicted living stature, in a Thai population, using the female MIDB calcaneal simple regression equation. 


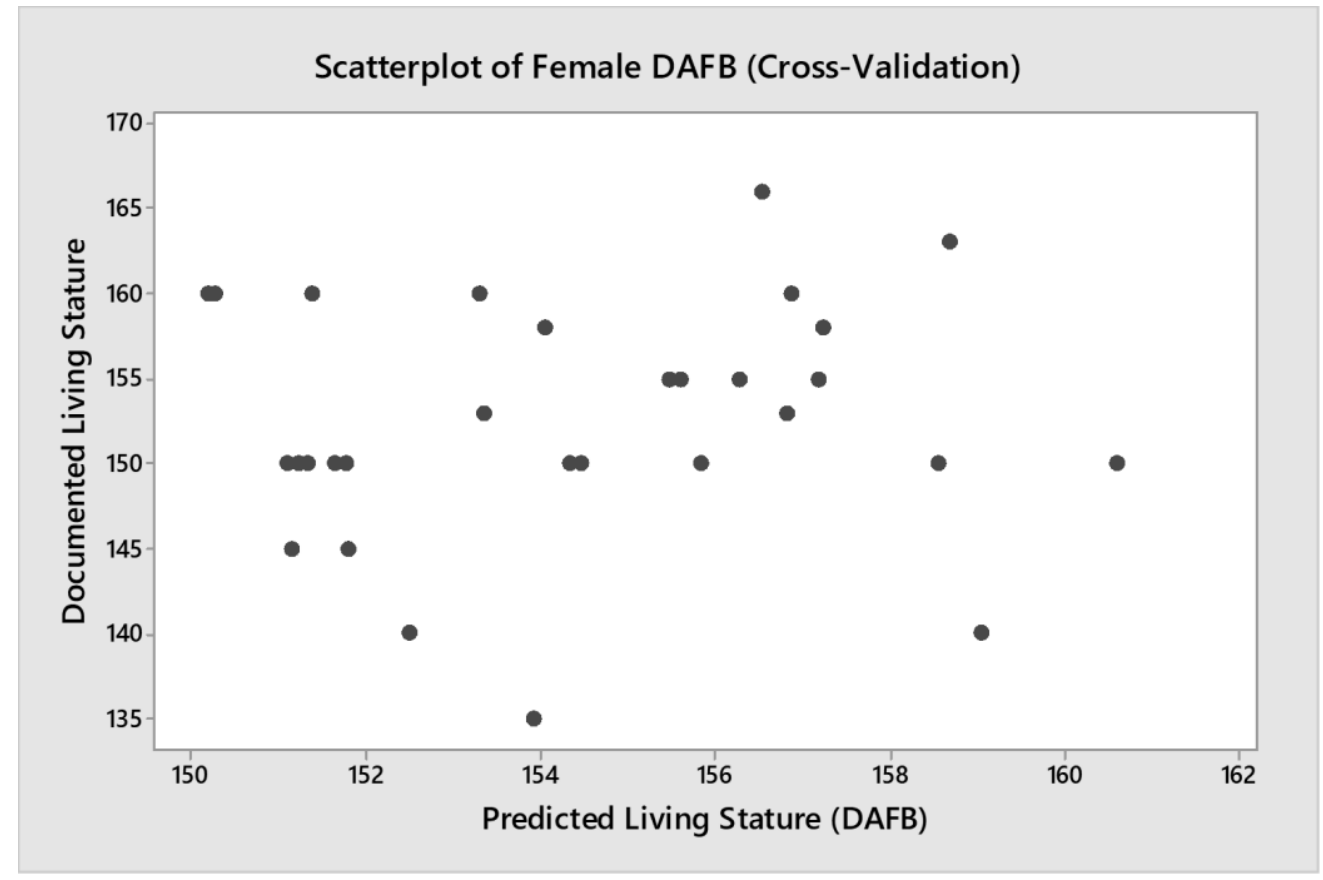

Appendix E12: Simple scatterplot illustrating the correlation between documented and predicted living stature, in a Thai population, using the female DAFB calcaneal simple regression equation.

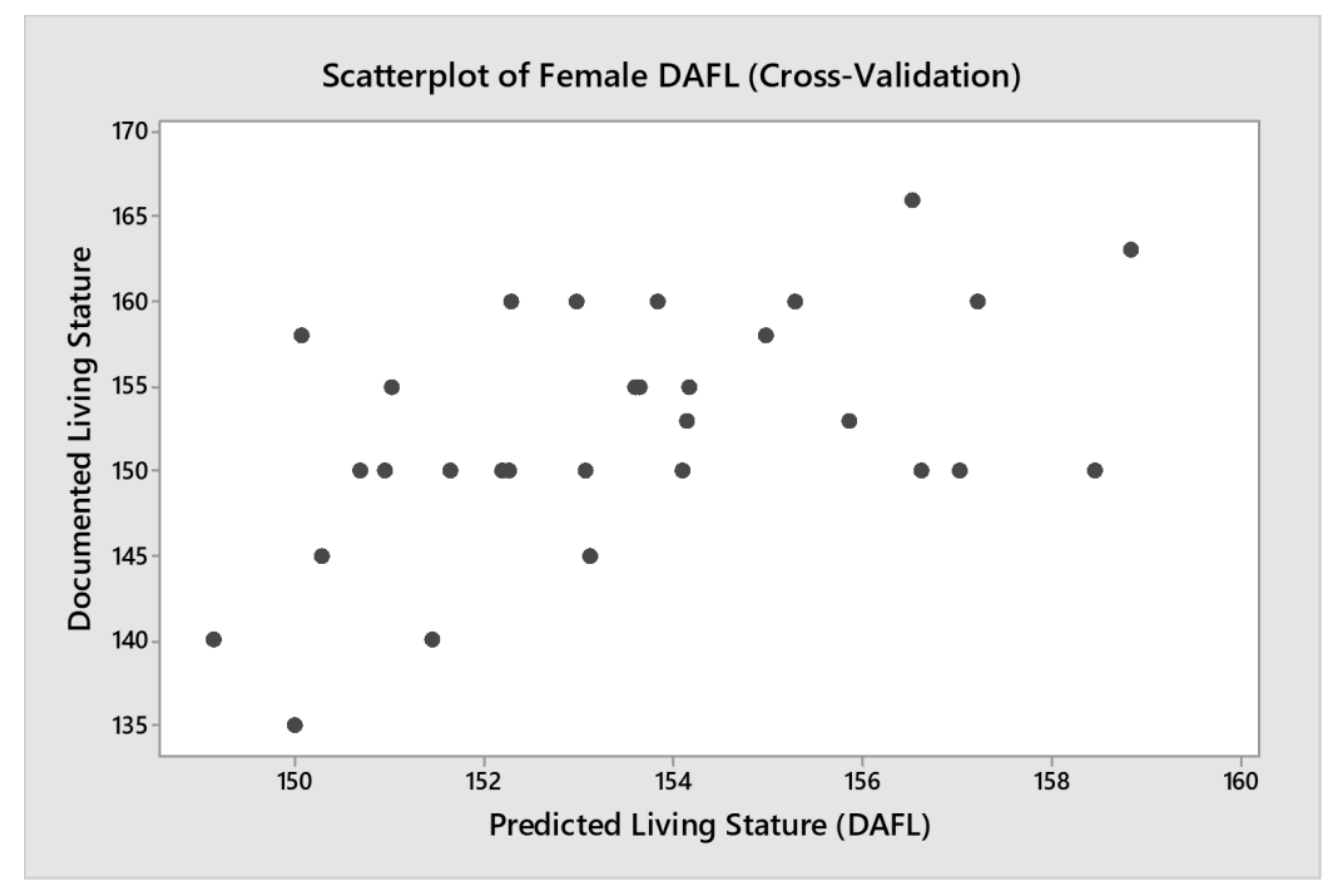

Appendix E13: Simple scatterplot illustrating the correlation between documented and predicted living stature, in a Thai population, using the female DAFL calcaneal simple regression equation. 


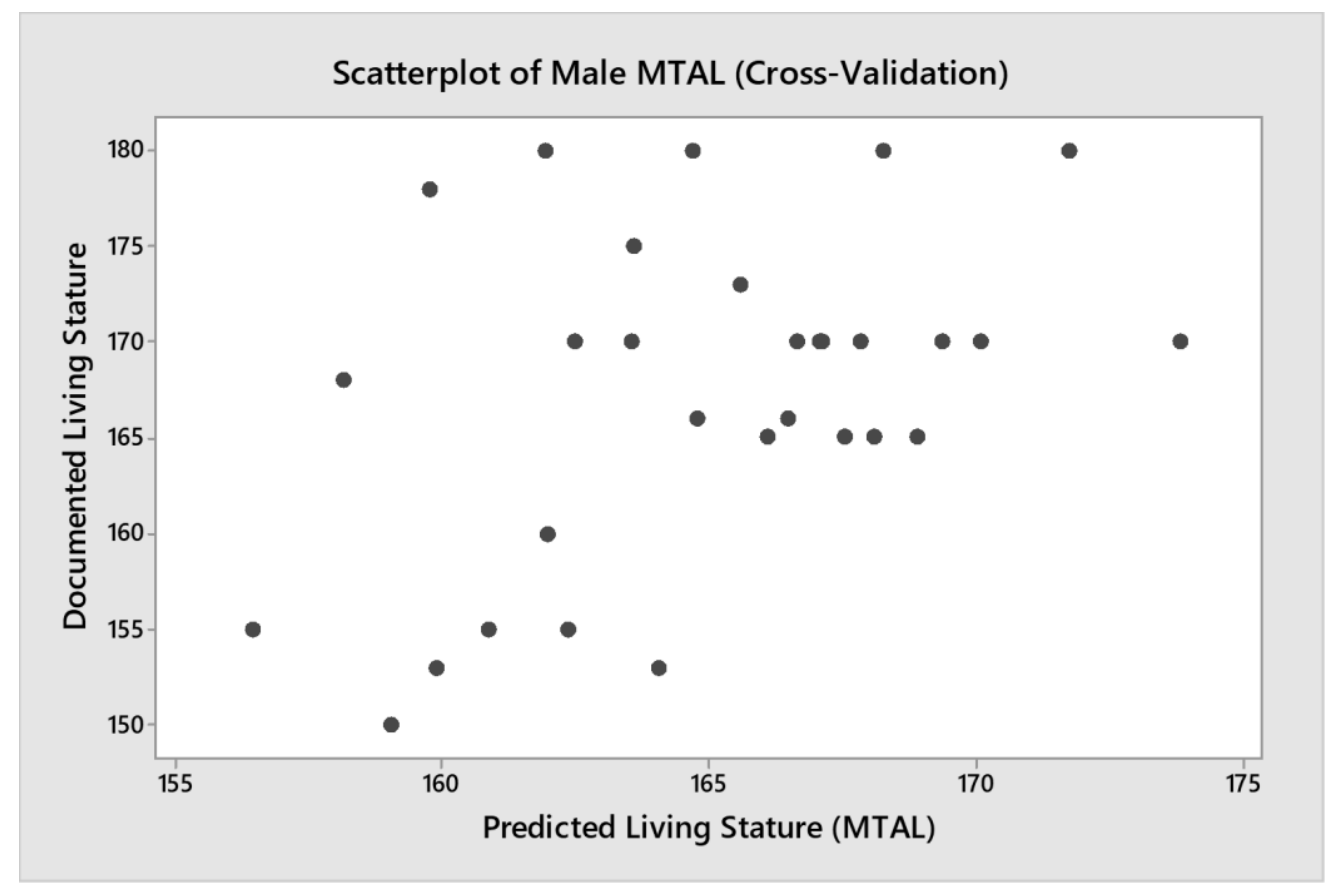

Appendix E14: Simple scatterplot illustrating the correlation between documented and predicted living stature, in a Thai population, using the male MTAL talar simple regression equation.

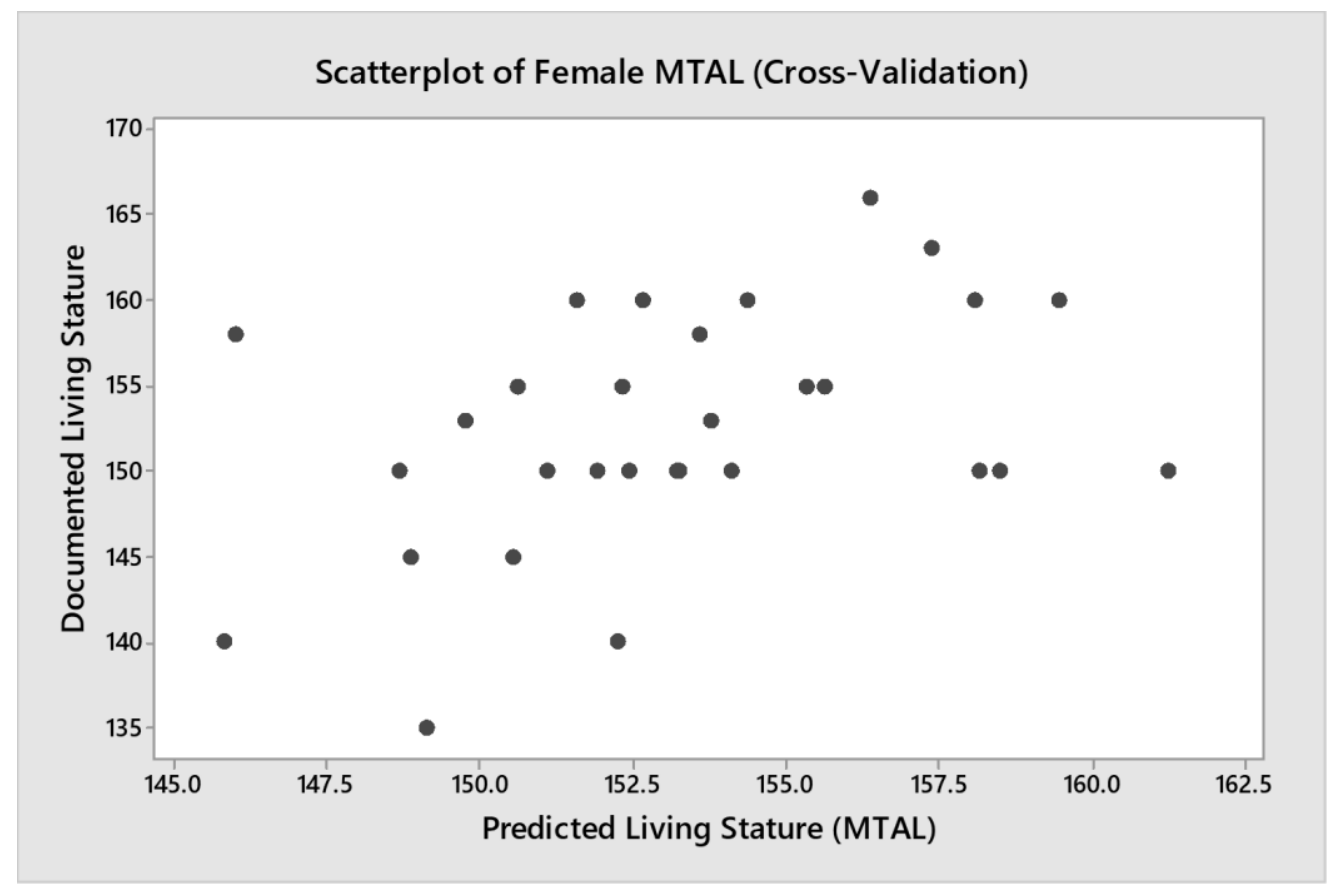

Appendix E15: Simple scatterplot illustrating the correlation between documented and predicted living stature, in a Thai population, using the female MTAL talar simple regression equation. 


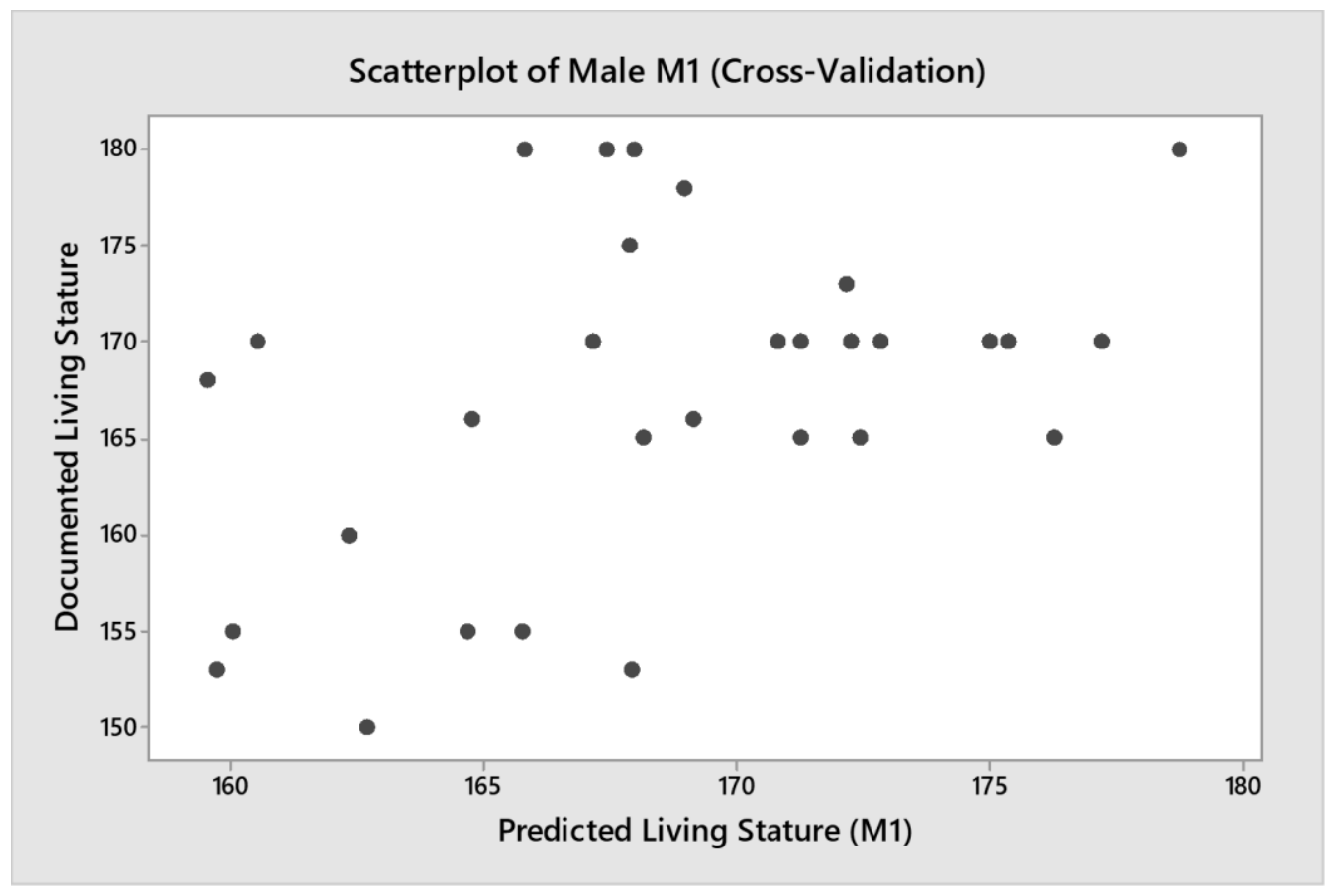

Appendix E16: Simple scatterplot illustrating the correlation between documented and predicted living stature, in a Thai population, using the male direct multiple regression equation 1.

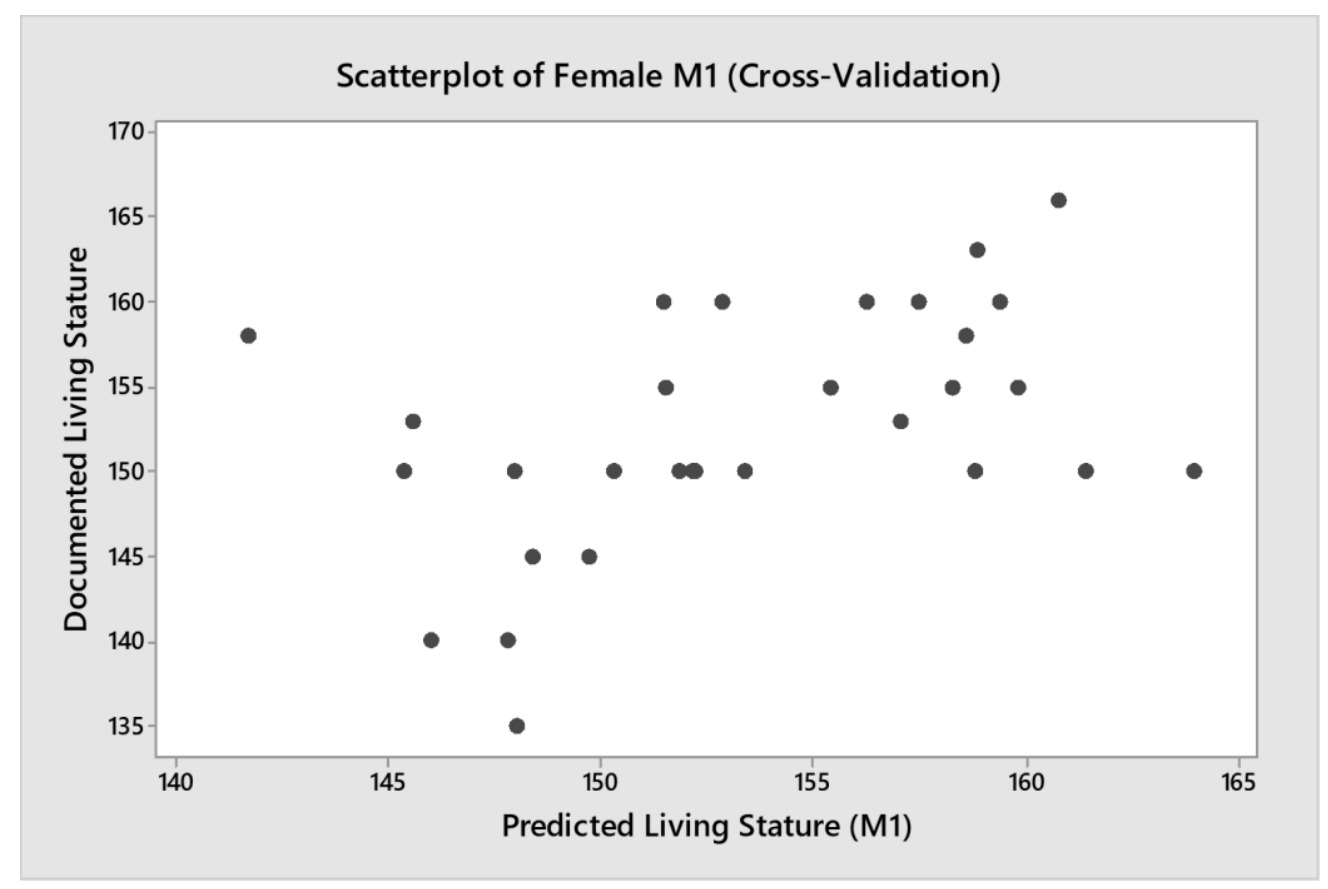

Appendix E17: Simple scatterplot illustrating the correlation between documented and predicted living stature, in a Thai population, using the female direct multiple regression equation 1. 


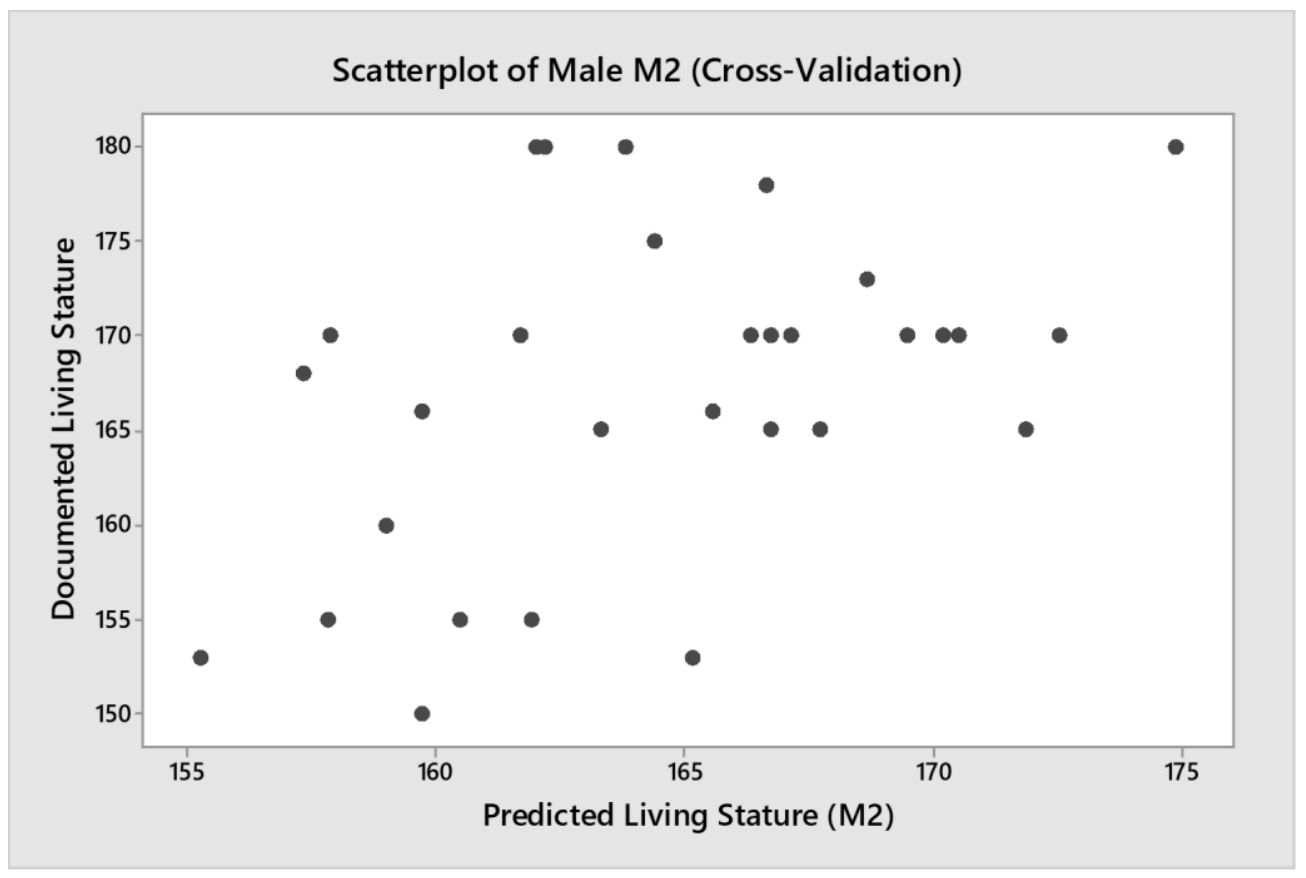

Appendix E18: Simple scatterplot illustrating the correlation between documented and predicted living stature, in a Thai population, using the male direct multiple regression equation 2.

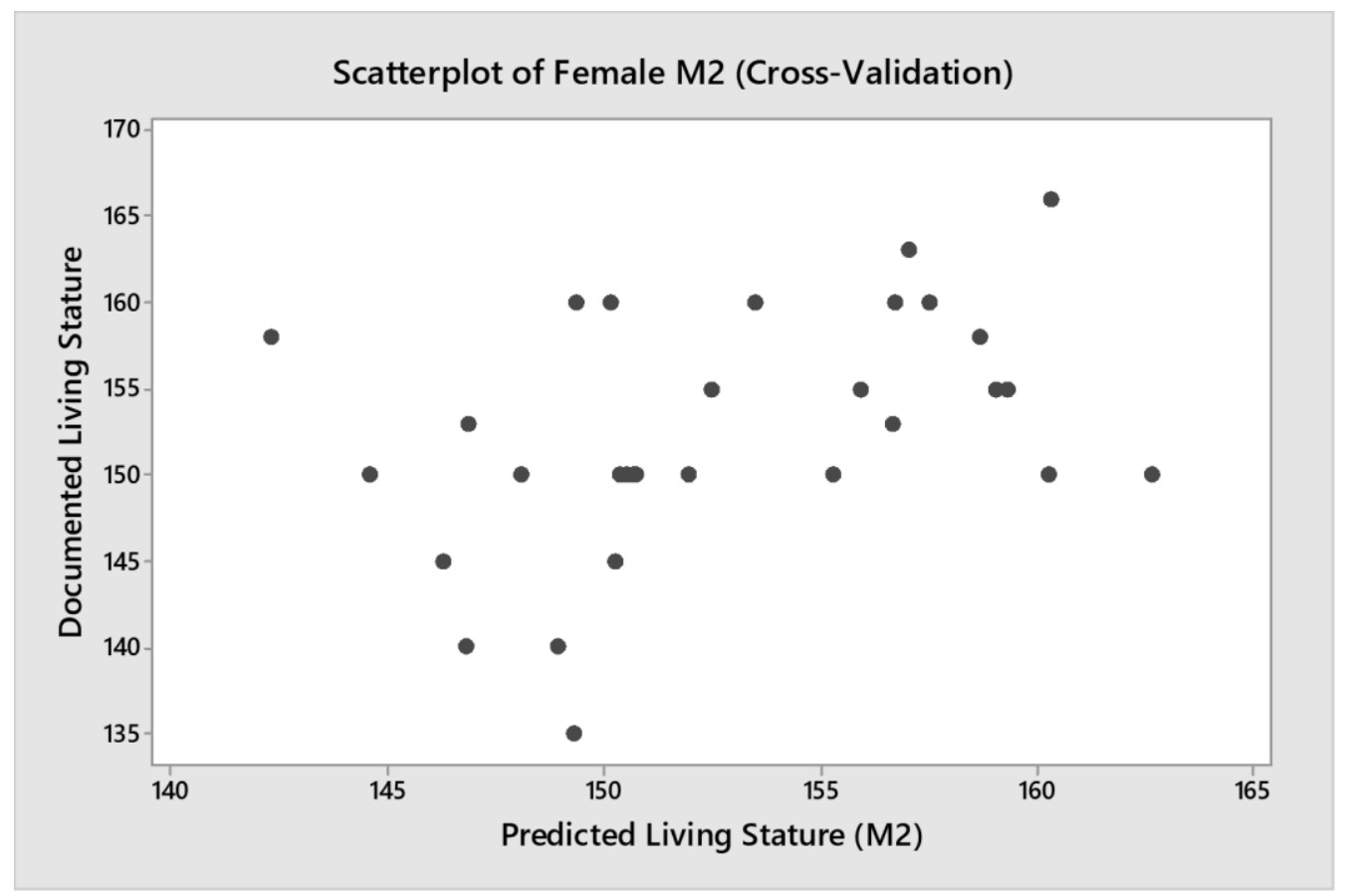

Appendix E19: Simple scatterplot illustrating the correlation between documented and predicted living stature, in a Thai population, using the female direct multiple regression equation 2 . 


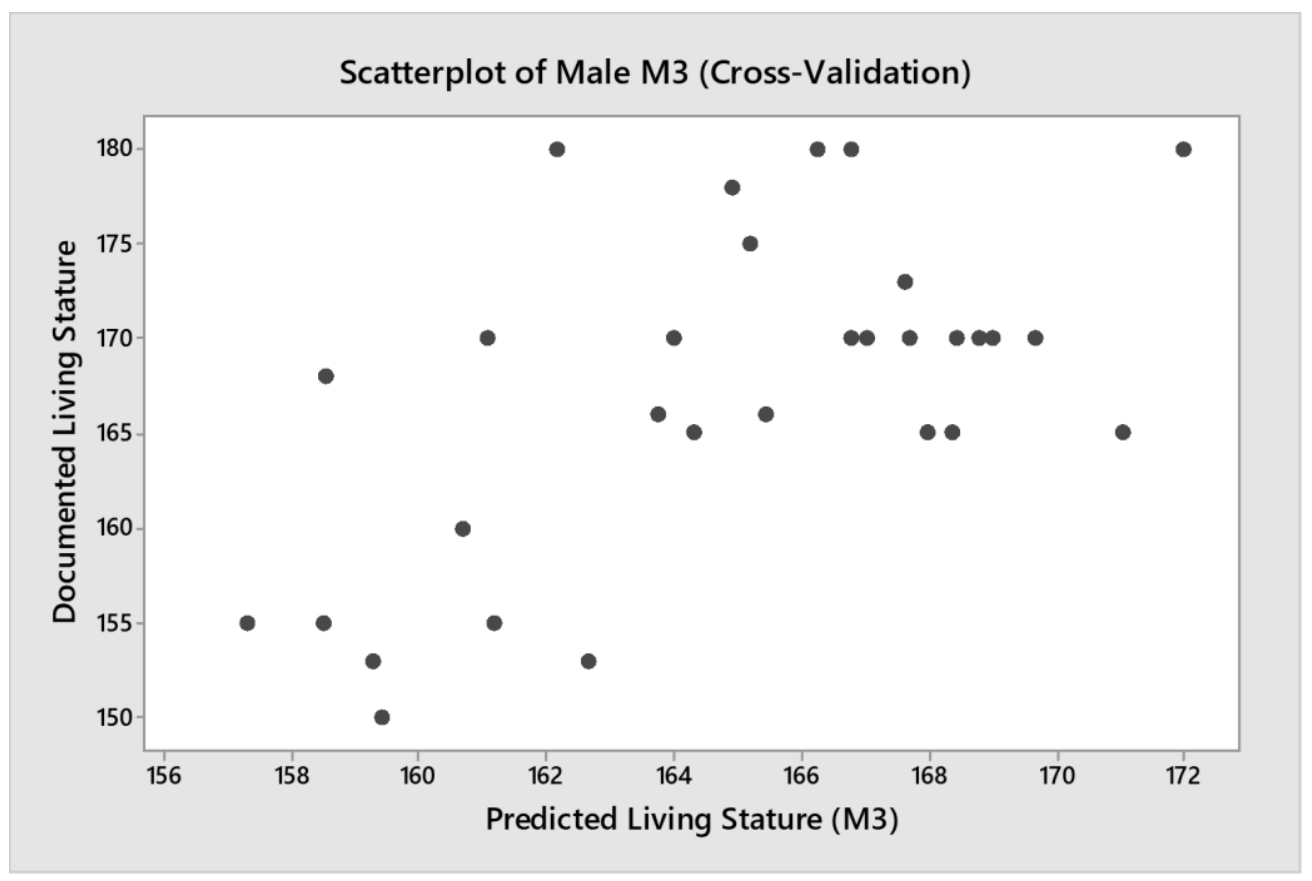

Appendix E20: Simple scatterplot illustrating the correlation between documented and predicted living stature, in a Thai population, using the male direct multiple regression equation 3.

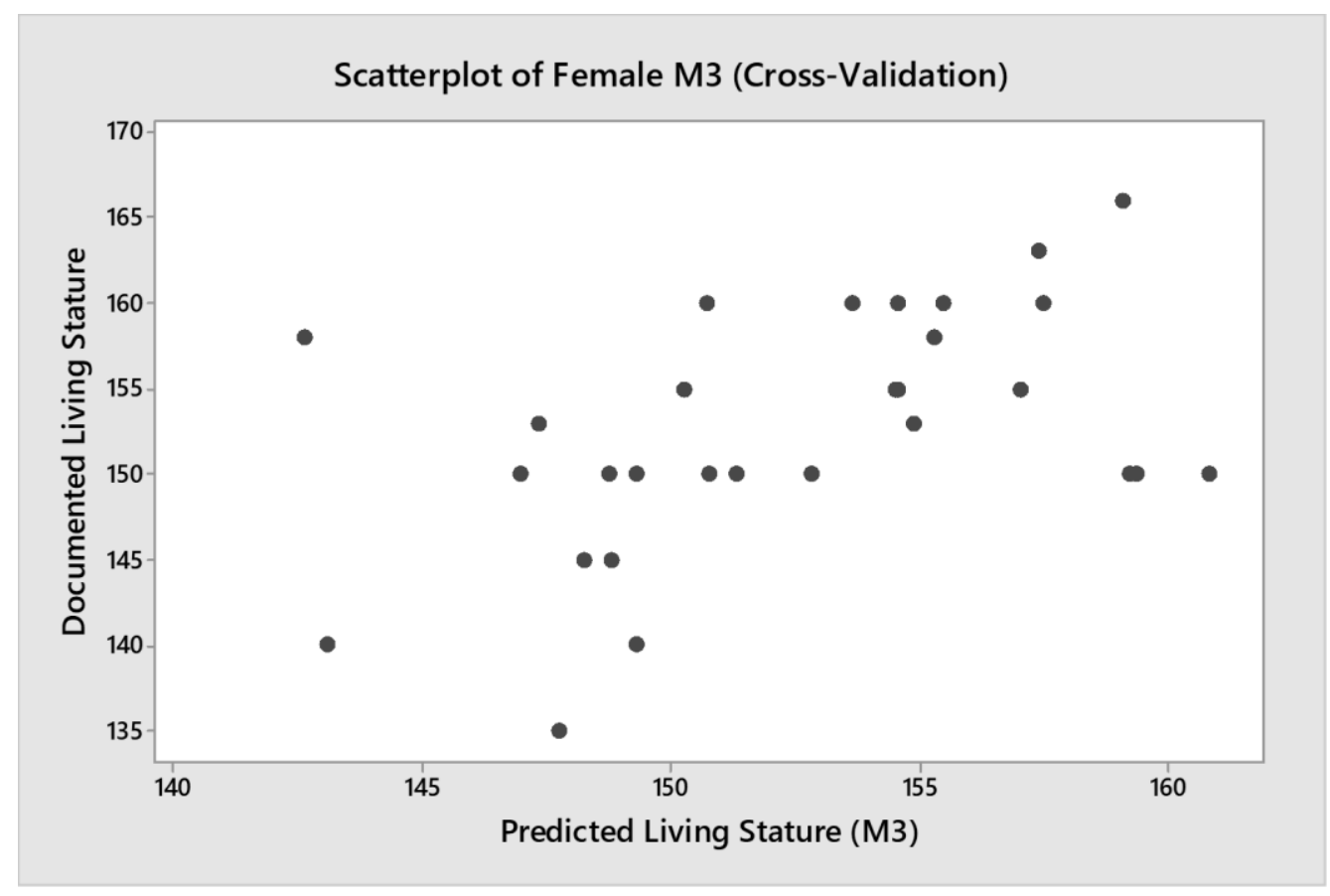

Appendix E21: Simple scatterplot illustrating the correlation between documented and predicted living stature, in a Thai population, using the female direct multiple regression equation 3. 


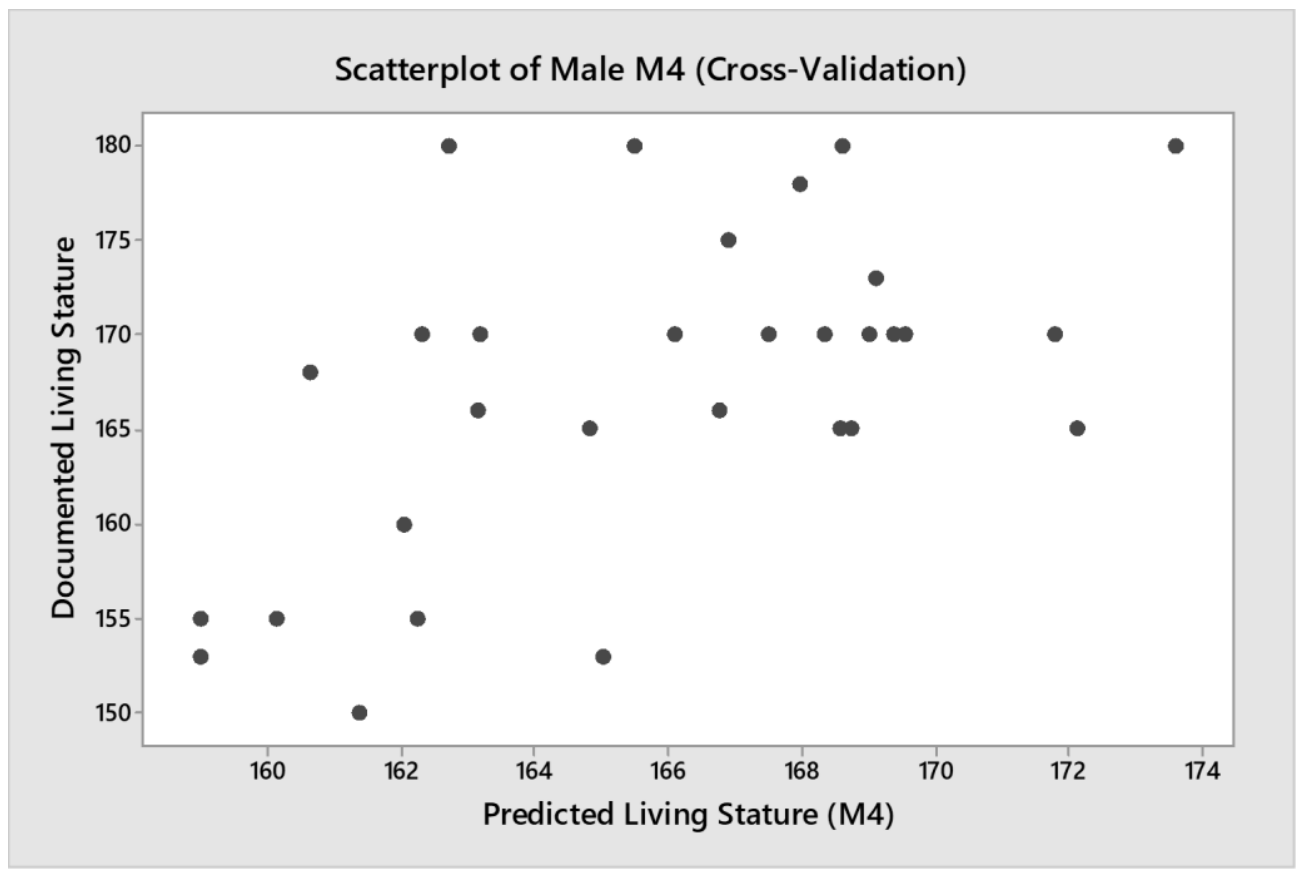

Appendix E22: Simple scatterplot illustrating the correlation between documented and predicted living stature, in a Thai population, using the male direct multiple regression equation 4.

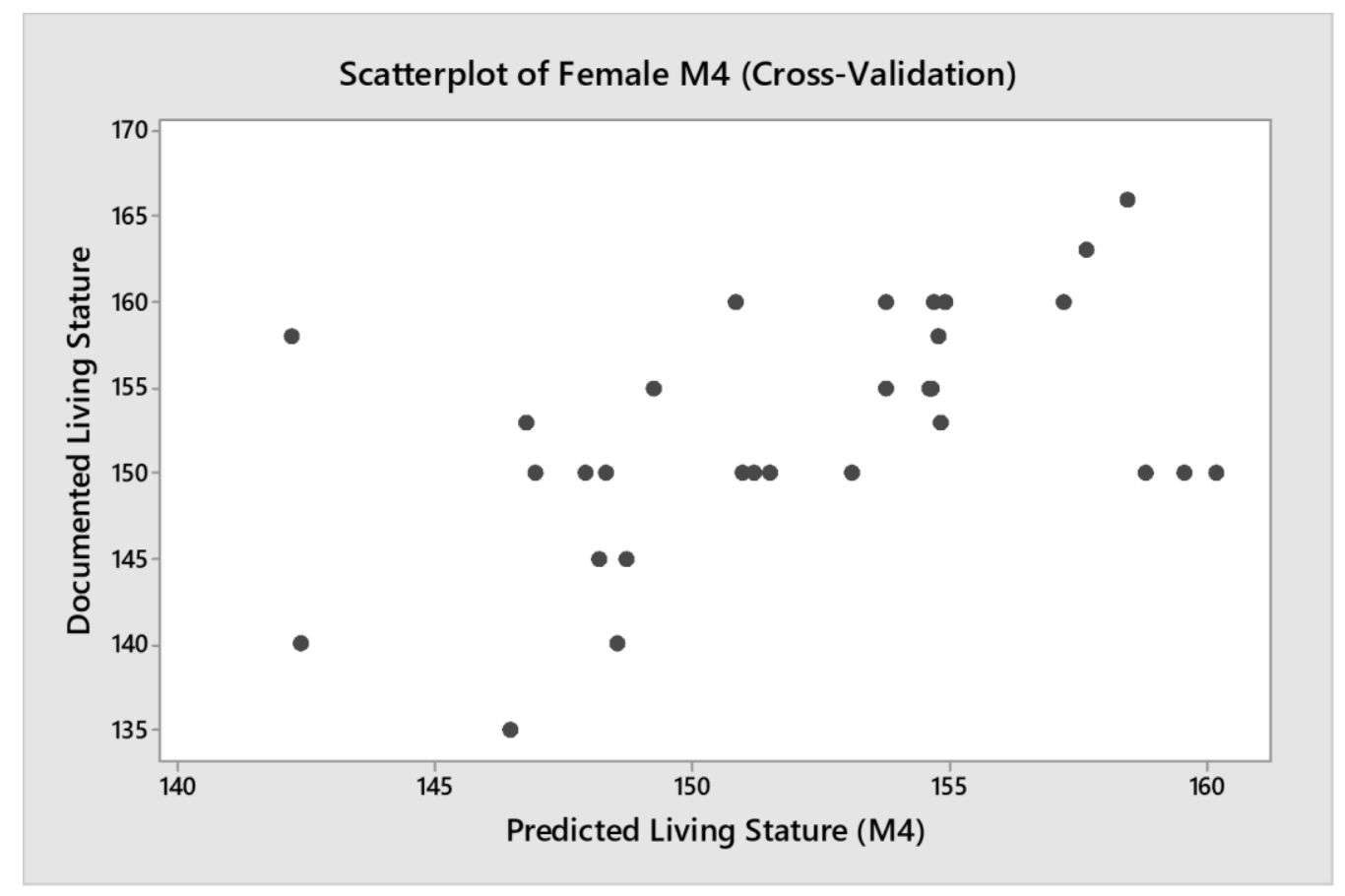

Appendix E23: Simple scatterplot illustrating the correlation between documented and predicted living stature, in a Thai population, using the female direct multiple regression equation 4. 


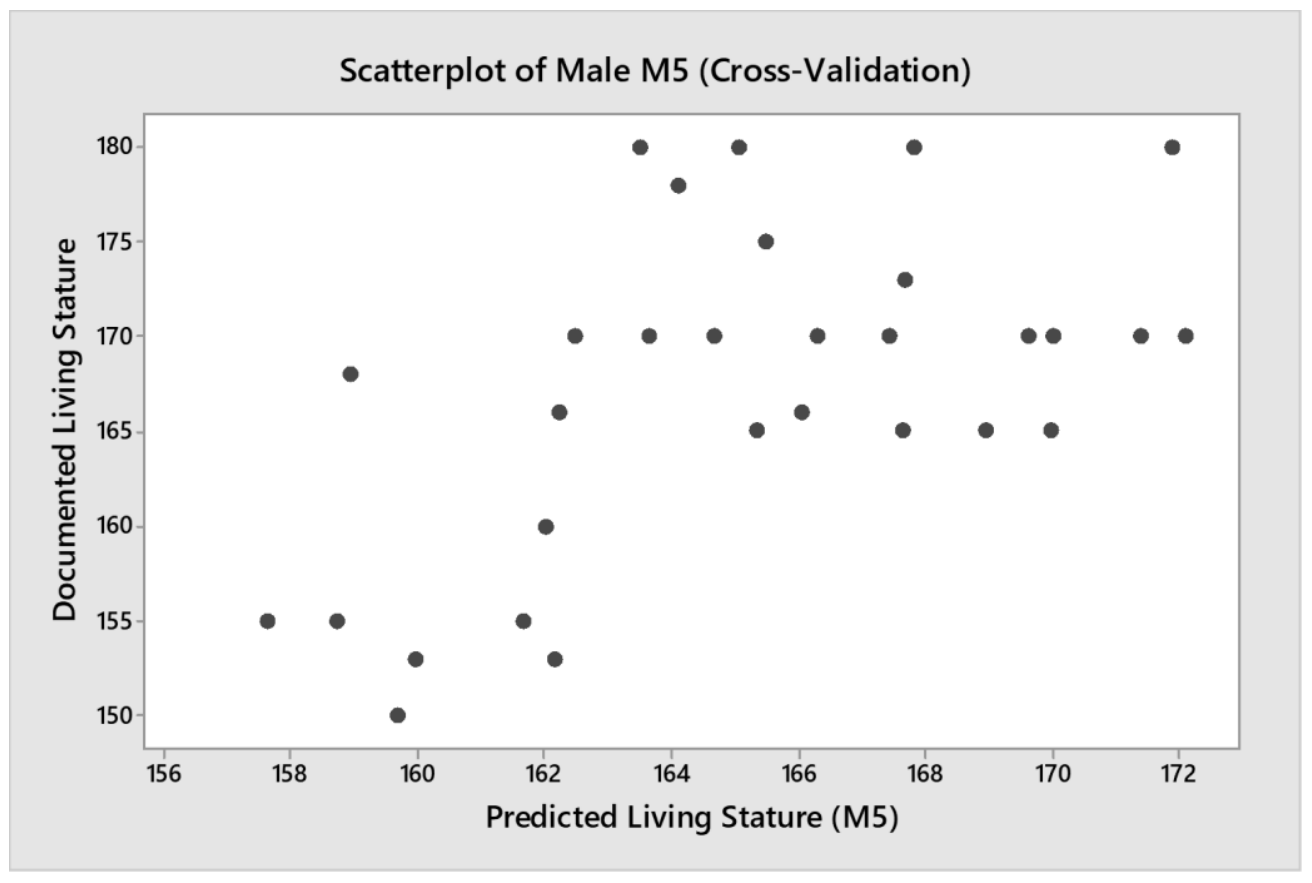

Appendix E24: Simple scatterplot illustrating the correlation between documented and predicted living stature, in a Thai population, using the male stepwise multiple regression equation 5 .

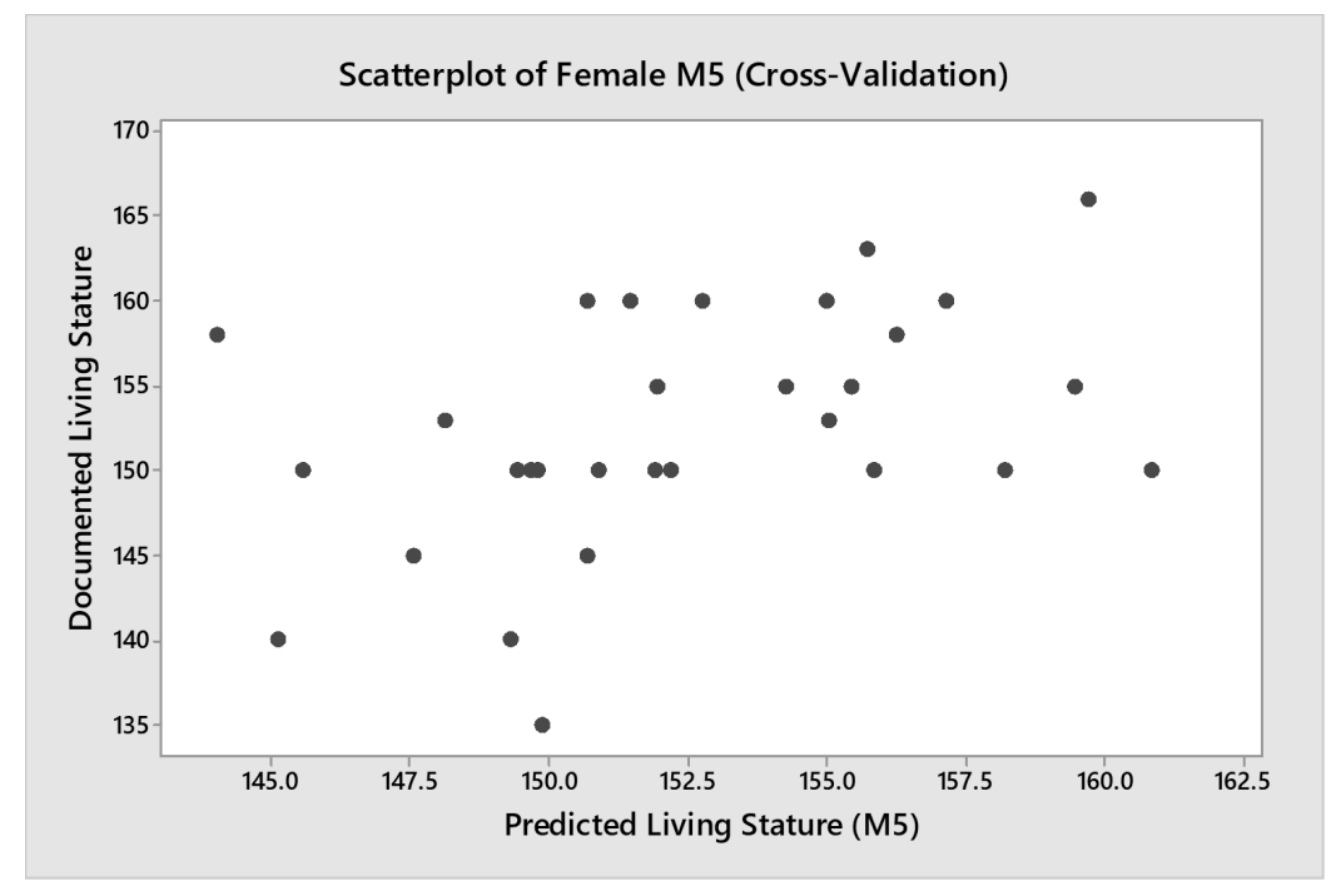

Appendix E25: Simple scatterplot illustrating the correlation between documented and predicted living stature, in a Thai population, using the female stepwise multiple regression equation 5 . 